\title{
OSW Saponins: Facile Synthesis Toward a New Type of Structures with Potent Antitumor Activities
}

\author{
Bingfeng Shi, ${ }^{\dagger}$ Pingping Tang, ${ }^{\dagger}$ Xiaoyi Hu, ${ }^{\ddagger}$ Jun O. Liu, ${ }^{\ddagger}$ Biao $\mathrm{Yu}^{*}{ }^{\dagger}$
}

\footnotetext{
† State Key Laboratory of Bioorganic and Natural Products Chemistry, Shanghai Institute of Organic Chemistry, Chinese Academy of Sciences, 354 Fenglin Road, Shanghai 200032, China. Fax: (0086)-21-64166128; Email: byu@mail.sioc.ac.cn

${ }^{\ddagger}$ Department of Pharmacology and Department of Neuroscience, Johns Hopkins School of Medicine, 725 North Wolfe Street, Baltimore, MD 21205, USA
}

\section{Supporting Information I:}

Experimental procedures (for synthesis and cell proliferation assay), analytical data (for new compounds), and ${ }^{1} \mathrm{H}$ and ${ }^{13} \mathrm{C}$ NMR spectra (for selected compounds) 


\section{S2-S5 List of Contents}

\section{S6-S31 Experimental Section}

S6 Materials and methods in cell proliferation assay

S6 General remarks for synthesis

S7 16-Bromo-3ß-(tert-butyldiphenylsiloxy)-5-androsten-17-one (3)

S7 16 16 -Hydroxy-3 $\beta$-(tert-butyldiphenylsiloxy)-5-androsten-17-one (5)

S8 16 $\alpha$-(tert-Butyldimethylsiloxy)-3 $\beta$-(tert-butyldiphenylsiloxy)-5-androsten-17-one (7)

S8 Typical procedure for aldol reaction under Conditions A

S9 (20S)-Ethyl

$3 \beta$-(tert-butyldiphenylsiloxy)-16 $\alpha, 17 \alpha$-dihydroxy-20-methyl-22-oate (8a)

S9 (20S)-Ethyl 3 $\beta$-(tert-butyldiphenylsiloxy)-16 $\alpha$-(tert-butyldimethylsiloxy) -17 $\alpha$-hydroxy-20-methyl-22-oate (10a)

S10 Typical procedure for aldol reaction under Conditions B

S10 (20R)-Ethyl

$3 \beta$-(tert-butyldiphenylsiloxy)-16 $\alpha, 17 \alpha$-dihydroxy-20-methyl-22-oate (8b)

S11 (20S)-Ethyl 3 $\beta$-(tert-butyldiphenylsiloxy)-16 $\alpha, 17 \alpha$-isopropylidenedioxy20-methyl-22-oate (13)

S11 Weinreb amide $\mathbf{1 4}$

S12 Methyl ketone $\mathbf{1 5}$

S12 Butyl ketone $\mathbf{1 6}$

S13 Isoamyl ketone $\mathbf{1 7}$

S13 Compound $\mathbf{1 8}$

S14 Compound 19

S14 22-Aldehyde 23

S14 Thioacetal 24

S15 5,16,20(22)-Triene 29

S16 Typical procedure for aldol reaction under Conditions $C$

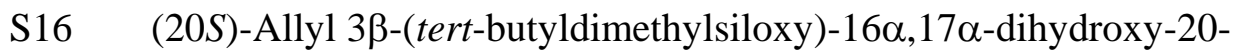
methyl-22-oate (41)

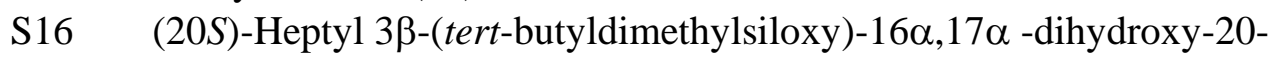
methyl-22-oate (42)

S17 (20S)-Octadecyl 3 $\beta$-(tert-butyldimethylsiloxy)-16 $\alpha, 17 \alpha$-dihydroxy-20methyl-22-oate (43)

S17 Dodecyl 3 $\beta$-(tert-butyldimethylsiloxy)-16 $\alpha, 17 \alpha$-dihydroxy-22-oate (44)

S18 (20S)-Isobutyl 3 $\beta$-(tert-butyldimethylsiloxy)-16 $\alpha, 17 \alpha$-dihydroxy-20methyl-22-thioate (45)

S18 General procedure for preparation of 16-ketones 47-48 and 50-52

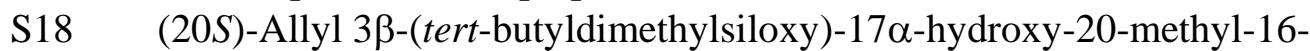
one-22-oate (47)

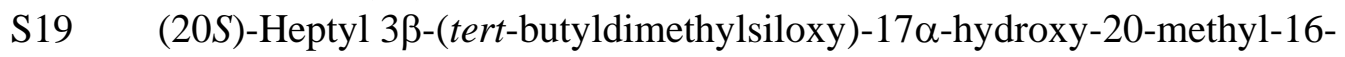
one-22-oate (48)

S19 (20S)-Octadecyl 3 $\beta$-(tert-butyldimethylsiloxy)-17 $\alpha$-hydroxy-20-methyl16-one-22-oate (50) 
S28

S29

S29

S30

S31

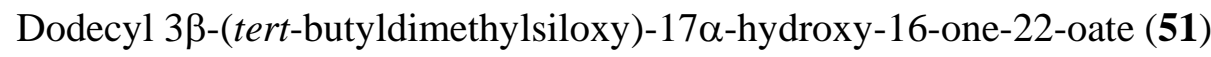
(20S)-Isobutyl 3 $\beta$-(tert-butyldimethylsiloxy)-17 $\alpha$-hydroxy-20-methyl16-one-22-thioate (52)

General procedure for preparation of $16 \alpha, 17 \beta$-trans diols 53-55 and 57-59

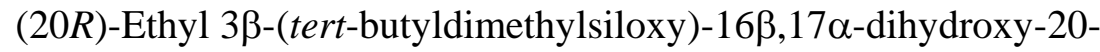
methyl-22-oate (53)

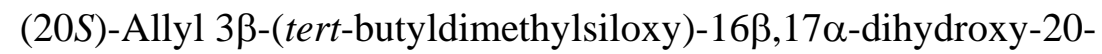
methyl-22-oate (54)

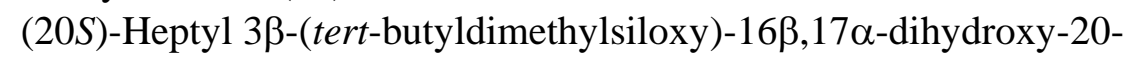
methyl-22-oate (55)

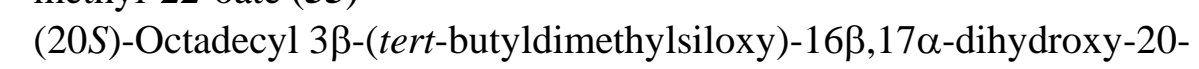
methyl-22-oate (57)

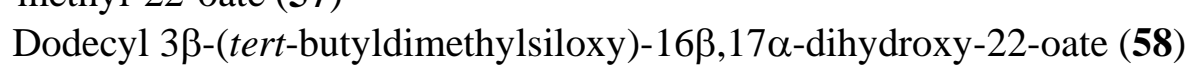

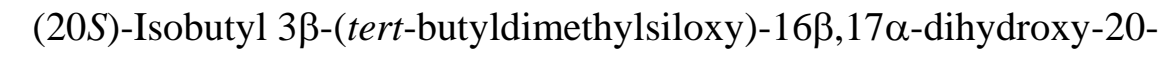
methyl-22-thioate (59)

General procedure for preparation of glycosides 61-63 and 65-67

Protected (20R)-ethyl-23-oxa-OSW-1 61

Protected (20S)-allyl-23-oxa-OSW-1 62

Protected (20S)-heptyl-23-oxa-OSW-1 63

Protected (20S)-octadecyl-23-oxa-OSW-1 65

Protected dodecyl-21-nor-23-oxa-OSW-1 66

Protected (20S)-23-thio-OSW-1 67

General procedure for preparation of $\mathbf{6 9}, \mathbf{7 1}$, and $\mathbf{7 3 - 7 5}$ by deprotection

(20R)-Ethyl-23-oxa-OSW-1 69

(20S)-Heptyl-23-oxa-OSW-1 71

(20S)-Octadecyl-23-oxa-OSW-1 73

Dodecyl-23-oxa-OSW-1 74

23-Thio-OSW-1 75

Compound 77

Protected 23-Aza-OSW-1 analogue 79

23-Aza-OSW-1 analogue 81

Compound 85

\section{S32-194 ${ }^{1} \mathrm{H}$ and ${ }^{13} \mathrm{C}$ NMR spectra for selected compounds}

S32 ${ }^{1} \mathrm{H}$ NMR of compound $2 \quad$ S43

S33 $\quad{ }^{13} \mathrm{C}$ NMR of compound $2 \quad$ S44

S34 ${ }^{1} \mathrm{H}$ NMR of compound $3(16 \alpha) \quad$ S45

S35 $\quad{ }^{1} \mathrm{H}$ NMR of compound $3(16 \beta) \quad$ S46

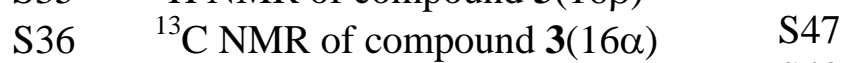

S37 $\quad{ }^{1} \mathrm{H}$ NMR of compound $4(16 \alpha) \quad$ S48

S38 $\quad{ }^{1} \mathrm{H}$ NMR of compound $4(16 \beta) \quad$ S49

S39 $\quad{ }^{13} \mathrm{C}$ NMR of compound $4(16 \alpha) \quad$ S50

S40 ${ }^{1} \mathrm{H}$ NMR of compound $5 \quad$ S51

S41 ${ }^{13} \mathrm{C}$ NMR of compound $5 \quad$ S52

S42 ${ }^{1}$ H NMR of compound $6 \quad$ S53

${ }^{13} \mathrm{C}$ NMR of compound 6

${ }^{1} \mathrm{H}$ NMR of compound 7

${ }^{13} \mathrm{C}$ NMR of compound 7

${ }^{1} \mathrm{H}$ NMR of compound $\mathbf{8 a}$

${ }^{13} \mathrm{C}$ NMR of compound $\mathbf{8 a}$

${ }^{1} \mathrm{H}$ NMR of compound $\mathbf{8 b}$

${ }^{13} \mathrm{C}$ NMR of compound $\mathbf{8 b}$

${ }^{1} \mathrm{H}$ NMR of compound 9a

${ }^{13} \mathrm{C}$ NMR of compound $9 \mathbf{a}$

${ }^{1} \mathrm{H}$ NMR of compound $\mathbf{9 b}$

${ }^{13} \mathrm{C}$ NMR of compound $\mathbf{9 b}$ 


\begin{tabular}{|c|c|c|c|}
\hline S54 & ${ }^{1} \mathrm{H}$ NMR of compound $\mathbf{1 0 a}$ & S100 & ${ }^{1} \mathrm{H}$ NMR of compound 36 \\
\hline S55 & ${ }^{13} \mathrm{C}$ NMR of compound 10a & S101 & ${ }^{13} \mathrm{C}$ NMR of compound 36 \\
\hline S56 & ${ }^{1} \mathrm{H}$ NMR of compound 11a & S102 & ${ }^{1} \mathrm{H}$ NMR of OSW-1 \\
\hline S57 & ${ }^{13} \mathrm{C}$ NMR of compound 11a & S103 & ${ }^{13} \mathrm{C}$ NMR of $\mathrm{OSW}-1$ \\
\hline S58 & ${ }^{1} \mathrm{H}$ NMR of compound 12a & S104 & ${ }^{1} \mathrm{H}$ NMR of compound 37 \\
\hline S59 & ${ }^{13} \mathrm{C}$ NMR of compound $\mathbf{1 2 a}$ & S105 & ${ }^{13} \mathrm{C}$ NMR of compound 37 \\
\hline$S 60$ & ${ }^{1} \mathrm{H}$ NMR of compound $\mathbf{1 3}$ & S106 & ${ }^{1} \mathrm{H}$ NMR of compound 38 \\
\hline 61 & ${ }^{13} \mathrm{C}$ NMR of compound $\mathbf{1 3}$ & S107 & ${ }^{13} \mathrm{C}$ NMR of compound 38 \\
\hline S62 & ${ }^{1} \mathrm{H}$ NMR of compound 14 & S108 & ${ }^{1} \mathrm{H}$ NMR of compound 39 \\
\hline S63 & ${ }^{13} \mathrm{C}$ NMR of compound 14 & S109 & ${ }^{13} \mathrm{C}$ NMR of compound 39 \\
\hline S64 & ${ }^{1} \mathrm{H}$ NMR of compound 15 & S110 & ${ }^{1} \mathrm{H}$ NMR of compound $\mathbf{4 0}$ \\
\hline S65 & ${ }^{13} \mathrm{C}$ NMR of compound 15 & S111 & ${ }^{13} \mathrm{C}$ NMR of compound $\mathbf{4 0}$ \\
\hline S66 & ${ }^{1} \mathrm{H}$ NMR of compound $\mathbf{1 6}$ & S112 & ${ }^{1} \mathrm{H}$ NMR of compound 41 \\
\hline S67 & ${ }^{13} \mathrm{C}$ NMR of compound $\mathbf{1 6}$ & S113 & ${ }^{1} \mathrm{H}$ NMR of compound 42 \\
\hline S68 & ${ }^{1} \mathrm{H}$ NMR of compound $\mathbf{1 7}$ & S114 & ${ }^{13} \mathrm{C}$ NMR of compound 42 \\
\hline S69 & ${ }^{13} \mathrm{C}$ NMR of compound $\mathbf{1 7}$ & S115 & ${ }^{1} \mathrm{H}$ NMR of compound 43 \\
\hline S70 & ${ }^{1} \mathrm{H}$ NMR of compound $\mathbf{1 8}$ & S116 & ${ }^{1} \mathrm{H}$ NMR of compound 44 \\
\hline S71 & ${ }^{13} \mathrm{C}$ NMR of compound $\mathbf{1 8}$ & S117 & ${ }^{1} \mathrm{H}$ NMR of compound 45 \\
\hline$S 72$ & ${ }^{1} \mathrm{H}$ NMR of compound $\mathbf{1 9}$ & S118 & ${ }^{13} \mathrm{C}$ NMR of compound 45 \\
\hline S73 & ${ }^{13} \mathrm{C}$ NMR of compound 19 & S119 & ${ }^{1} \mathrm{H}$ NMR of compound 47 \\
\hline S74 & ${ }^{1} \mathrm{H}$ NMR of compound 20 & S120 & ${ }^{13} \mathrm{C}$ NMR of compound 47 \\
\hline S75 & ${ }^{13} \mathrm{C}$ NMR of compound 20 & S121 & ${ }^{1} \mathrm{H}$ NMR of compound $\mathbf{4 8}$ \\
\hline S76 & ${ }^{1} \mathrm{H}$ NMR of compound 21 & S122 & ${ }^{13} \mathrm{C}$ NMR of compound 48 \\
\hline S77 & ${ }^{13} \mathrm{C}$ NMR of compound 21 & S123 & ${ }^{1} \mathrm{H}$ NMR of compound 49 \\
\hline S78 & ${ }^{1} \mathrm{H}$ NMR of compound 22 & S124 & ${ }^{13} \mathrm{C}$ NMR of compound 49 \\
\hline S79 & ${ }^{13} \mathrm{C}$ NMR of compound 22 & S125 & ${ }^{1} \mathrm{H}$ NMR of compound 50 \\
\hline S80 & ${ }^{1} \mathrm{H}$ NMR of compound 23 & S126 & ${ }^{13} \mathrm{C}$ NMR of compound $\mathbf{5 0}$ \\
\hline S81 & ${ }^{13} \mathrm{C}$ NMR of compound 23 & S127 & ${ }^{1} \mathrm{H}$ NMR of compound 51 \\
\hline S82 & ${ }^{1} \mathrm{H}$ NMR of compound 24 & S128 & ${ }^{13} \mathrm{C}$ NMR of compound 51 \\
\hline S83 & ${ }^{13} \mathrm{C}$ NMR of compound 24 & S129 & ${ }^{1} \mathrm{H}$ NMR of compound 52 \\
\hline S84 & ${ }^{1} \mathrm{H}$ NMR of compound 25 & S130 & ${ }^{13} \mathrm{C}$ NMR of compound 52 \\
\hline S85 & ${ }^{13} \mathrm{C}$ NMR of compound 25 & S131 & ${ }^{1} \mathrm{H}$ NMR of compound 53 \\
\hline S86 & ${ }^{1} \mathrm{H}$ NMR of compound $\mathbf{2 6}$ & S132 & ${ }^{13} \mathrm{C}$ NMR of compound 53 \\
\hline S87 & ${ }^{1} \mathrm{H}$ NMR of compound 27 & S133 & ${ }^{1} \mathrm{H}$ NMR of compound 54 \\
\hline S88 & ${ }^{13} \mathrm{C}$ NMR of compound 27 & S134 & ${ }^{1} \mathrm{H}$ NMR of compound 55 \\
\hline S89 & ${ }^{1} \mathrm{H}$ NMR of compound 29 & S135 & ${ }^{13} \mathrm{C}$ NMR of compound 55 \\
\hline S90 & ${ }^{13} \mathrm{C}$ NMR of compound 29 & S136 & ${ }^{1} \mathrm{H}$ NMR of compound 56 \\
\hline S91 & ${ }^{1} \mathrm{H}$ NMR of compound $\mathbf{3 0}$ & S137 & ${ }^{13} \mathrm{C}$ NMR of compound 56 \\
\hline S92 & ${ }^{1} \mathrm{H}$ NMR of compound 31 & S138 & ${ }^{1} \mathrm{H}$ NMR of compound 57 \\
\hline S93 & ${ }^{13} \mathrm{C}$ NMR of compound 31 & S139 & ${ }^{1} \mathrm{H}$ NMR of compound 58 \\
\hline S94 & ${ }^{1} \mathrm{H}$ NMR of compound 32 & S140 & ${ }^{13} \mathrm{C}$ NMR of compound 58 \\
\hline S95 & ${ }^{13} \mathrm{C}$ NMR of compound 32 & S141 & ${ }^{1} \mathrm{H}$ NMR of compound 59 \\
\hline S96 & ${ }^{1} \mathrm{H}$ NMR of compound 34 & S142 & ${ }^{13} \mathrm{C}$ NMR of compound 59 \\
\hline S97 & ${ }^{13} \mathrm{C}$ NMR of compound 34 & S143 & ${ }^{1} \mathrm{H}$ NMR of compound $\mathbf{6 0}$ \\
\hline S98 & ${ }^{1} \mathrm{H}$ NMR of compound 35 & S144 & ${ }^{13} \mathrm{C}$ NMR of compound $\mathbf{6 0}$ \\
\hline S99 & ${ }^{13} \mathrm{C}$ NMR of compound 35 & S145 & ${ }^{1} \mathrm{H}$ NMR of compound $\mathbf{6 2}$ \\
\hline
\end{tabular}




$\begin{array}{ll}\text { S146 } & { }^{1} \mathrm{H} \text { NMR of compound } \mathbf{6 3} \\ \text { S147 } & { }^{13} \mathrm{C} \text { NMR of compound } \mathbf{6 3} \\ \text { S148 } & { }^{1} \mathrm{H} \text { NMR of compound } \mathbf{6 4} \\ \text { S149 } & { }^{13} \mathrm{C} \text { NMR of compound } \mathbf{6 4} \\ \text { S150 } & { }^{1} \mathrm{H} \text { NMR of compound } \mathbf{6 5} \\ \text { S151 } & { }^{13} \mathrm{C} \text { NMR of compound } \mathbf{6 5} \\ \text { S152 } & { }^{1} \mathrm{H} \text { NMR of compound } \mathbf{6 6} \\ \text { S153 } & { }^{1} \mathrm{H} \text { NMR of compound } \mathbf{6 7} \\ \text { S154 } & { }^{1} \mathrm{H} \text { NMR of compound } \mathbf{6 8} \\ \text { S155 } & { }^{13} \mathrm{C} \text { NMR of compound } \mathbf{6 8} \\ \text { S156 } & { }^{1} \mathrm{H} \text { NMR of compound } \mathbf{6 9} \\ \text { S157 } & { }^{13} \mathrm{C} \text { NMR of compound } \mathbf{6 9} \\ \text { S158 } & { }^{1} \mathrm{H} \text { NMR of compound 70 } \\ \text { S159 } & { }^{13} \mathrm{C} \text { NMR of compound } \mathbf{7 0} \\ \text { S160 } & { }^{1} \mathrm{H} \text { NMR of compound } \mathbf{7 1} \\ \text { S161 } & { }^{13} \mathrm{C} \text { NMR of compound } \mathbf{7 1} \\ \text { S162 } & { }^{1} \mathrm{H} \text { NMR of compound } 72 \\ \text { S163 } & { }^{13} \mathrm{C} \text { NMR of compound } \mathbf{7 2} \\ \text { S164 } & { }^{1} \mathrm{H} \text { NMR of compound 73 } \\ \text { S165 } & { }^{13} \mathrm{C} \text { NMR of compound } \mathbf{7 3} \\ \text { S166 } & { }^{1} \mathrm{H} \text { NMR of compound } \mathbf{7 4}\end{array}$

S167 $\quad{ }^{13} \mathrm{C}$ NMR of compound 74

S168 ${ }^{1} \mathrm{H}$ NMR of compound 75

S169 $\quad{ }^{13} \mathrm{C}$ NMR of compound 75

S170 ${ }^{1} \mathrm{H}$ NMR of compound 76

S171 ${ }^{13} \mathrm{C}$ NMR of compound 76

S172 ${ }^{1} \mathrm{H}$ NMR of compound 77

S173 ${ }^{13} \mathrm{C}$ NMR of compound 77

S174 $\quad{ }^{1} \mathrm{H}$ NMR of compound 78

S175 ${ }^{13} \mathrm{C}$ NMR of compound 78

S176 ${ }^{1} \mathrm{H}$ NMR of compound 79

S177 ${ }^{13} \mathrm{C}$ NMR of compound $\mathbf{7 9}$

S178 ${ }^{1} \mathrm{H}$ NMR of compound $\mathbf{8 0}$

S179 ${ }^{13} \mathrm{C}$ NMR of compound $\mathbf{8 0}$

S180 $\quad{ }^{1}$ H NMR of compound $\mathbf{8 1}$

S181 ${ }^{13} \mathrm{C}$ NMR of compound $\mathbf{8 1}$

S182 ${ }^{1} \mathrm{H}$ NMR of compound 83

S183 ${ }^{13} \mathrm{C}$ NMR of compound 83

S184 ${ }^{1} \mathrm{H}$ NMR of compound $\mathbf{8 4}$

S185 ${ }^{13} \mathrm{C}$ NMR of compound $\mathbf{8 4}$

S186 ${ }^{1} \mathrm{H}$ NMR of compound 85

S187 ${ }^{13} \mathrm{C}$ NMR of compound 85 


\section{Experimental Section}

\section{Materials and Methods in cell proliferation assay:}

Cells were grown in a humidified incubator at $37{ }^{\circ} \mathrm{C}$ in an atmosphere of $5 \% \mathrm{CO}_{2}$. Human HeLa, JurKat leukemia T cells, and MCF-7 cells were obtained from ATCC. HeLa cells were cultured in DMEM with 10\% fetal bovine serum (FBS) and 50 units/mL penicillin plus $50 \mu \mathrm{g} / \mathrm{mL}$ streptomycin (P/S). The human Jurkat leukemia $\mathrm{T}$ cells and human MCF-7 cells were cultured in RPMI 60 medium with $10 \%$ fetal bovine serum (FBS) and 50 units/mL penicillin plus $50 \mu \mathrm{g} / \mathrm{mL}$ streptomycin (P/S).

Cell proliferation assay was adapted from a literature procedure (Zhang, Y.; Griffith, E. C.; Sage, J.; Jacks, T.; Liu, J. O. Cell cycle inhibition by the anti-angiogenic agent TNP-470 is mediated by p53 and p21WAF1/CIP1. Proc. Natl. Acad. Sci. USA 2000, 97, 6427-6432). Briefly, proliferating cells were seeded into a 96-well plate and grown in the presence of varying concentrations of testing compounds or carrier vehicle (0.1\% DMSO) for $24 \mathrm{~h}$. Cells were pulsed with $\left[{ }^{3} \mathrm{H}\right]$-thymidine $\left(6.7 \mathrm{Ci} \mathrm{mmol}^{-1}, 1 \mu \mathrm{Ci}\right.$ per well) for an additional $6 \mathrm{~h}$ before harvested with a semiautomated cell harvester onto glass fiber papers for scintillation counting. All assays were performed in triplicate and presented as the mean numbers.

\section{General remarks for synthesis:}

All solvents were distilled prior to use except where noted. All reactions sensitive to moisture or oxygen were conducted under an atmosphere of nitrogen or argon using flame-dried or oven-dried $\left(170{ }^{\circ} \mathrm{C}\right)$ glassware. Crushed $4 \AA$ molecular sieves were activated by thorough flame-drying immediately prior to use.

Flash column chromatography was performed on silica gel H (10-40 $\mu)$. Analytical thin layer chromatography (TLC) was performed on glass plates pre-coated with a 0.25 mm thickness of silica gel. The TLC plates were visualized with UV light and/or by staining with ethanolic phosphomolybdic acid (PMA) or acidic methanol. NMR: Chemical shifts $(\delta)$ are given in ppm relative to TMS, coupling constants $(J)$ in Hz. 
For the synthetic procedure and analytical data for compounds 2, 4, 6, 9a, 9b, 11a, 12a, $25,27,37,38,39,40,46,49,56,60,64,68$, and 72 , see the Supporting Information in lit.15.

\section{6-Bromo-3 $\beta$-(tert-butyldiphenylsiloxy)-5-androsten-17-one (3)}

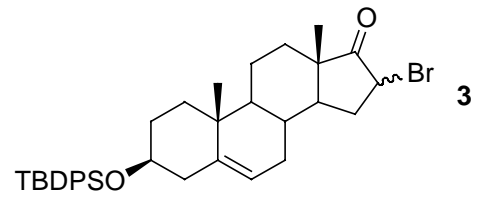

To a solution of 2 (3.09 g, $8.4 \mathrm{mmol})$ and imidazole (1.43 g, $21 \mathrm{mmol})$ in dry DMF (30 $\mathrm{mL}$ ) was added tert-butyldiphenylsilyl chloride (2.6 mL, $10.08 \mathrm{mmol}$ ) at rt. After stirring overnight, the suspension was diluted with $\mathrm{CH}_{2} \mathrm{Cl}_{2}$. The organic layer was washed with saturated $\mathrm{NaHCO}_{3}$ and brine, respectively, and then dried over $\mathrm{Na}_{2} \mathrm{SO}_{4}$, and filtered. The filtrate was concentrated in vacuo to give a residue, which was purified by a flash column chromatography (petroleum ether:EtOAc: $\mathrm{CH}_{2} \mathrm{Cl}_{2}, 34: 1: 2$ ) to afford a mixture of the 16-epimers 3 (4.83 g, 95\%) as a white foam. $[\alpha]^{24}=-7.4\left(c\right.$ 1.0, $\left.\mathrm{CHCl}_{3}\right) ;{ }^{1} \mathrm{H}$ NMR (300 MHz, $\left.\mathrm{CDCl}_{3}\right): \delta$ 7.69-7.33 (m, 10 H), 5.15 (m, $\left.1 \mathrm{H}\right), 4.53$ (m, $\left.1 \mathrm{H}\right), 3.54$ (m, $\left.1 \mathrm{H}\right), 1.07$ (s, $9 \mathrm{H}), 1.02$ (s, $3 \mathrm{H}), 0.91$ (s, $3 \mathrm{H}$ ); ${ }^{13} \mathrm{C}$ NMR (75 MHz, $\mathrm{CDCl}_{3}$ ): $\delta$ 213.2, 141.5, 135.7, 134.8, 134.6, 129.4, 127.7, 127.4, 120.0, 73.0, 49.9, 48.2, 47.4, 46.2, 37.0, 36.5, 34.1, 32.2, 31.7, 30.7, 30.5, 27.0, 26.5, 20.1, 19.4, 13.9; EIMS m/z (\%): 549 (47.5), 547 ( $\mathrm{M}^{+}-57$, 44.7), 199 (100); Anal. Calcd for $\mathrm{C}_{35} \mathrm{H}_{45} \mathrm{BrO}_{2} \mathrm{Si}$ : C, 69.40; H, 7.49; Found: C, 69.68; $\mathrm{H}$, 7.58. The $16 \beta$-isomer of 3: ${ }^{1} \mathrm{H}$ NMR (300 MHz, $\left.\mathrm{CDCl}_{3}\right): \delta 4.09(\mathrm{t}, J=9.1 \mathrm{~Hz}, 1 \mathrm{H}$ ), 2.64-2.55 (m, $1 \mathrm{H})$.

\section{6 $\alpha$-Hydroxy-3 $\beta$-(tert-butyldiphenylsiloxy)-5-androsten-17-one (5)}

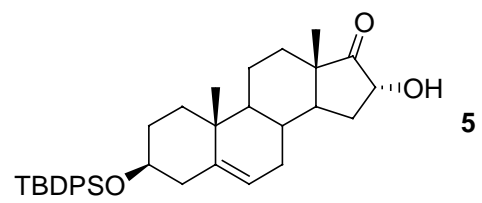

To a solution of 3 (460 mg, $0.76 \mathrm{mmol})$ in $\mathrm{CH}_{2} \mathrm{Cl}_{2}$ and DMF (10 mL, v/v, 2:3) was added $2 \mathrm{~mL}$ of $\mathrm{NaOH}$ (34 mg, $0.85 \mathrm{mmol}$ ) aqueous solution, and the mixture was allowed to 
stand at rt overnight. The mixture was poured into $5 \% \mathrm{HCl}$ solution and then extracted with $\mathrm{CH}_{2} \mathrm{Cl}_{2}$. The organic layer was washed with saturated $\mathrm{NaHCO}_{3}$ and brine and dried over $\mathrm{Na}_{2} \mathrm{SO}_{4}$. After evaporation of the solvent, the residue was purified by a flash column chromatography (petroleum ether:EtOAc: $\left.\mathrm{CH}_{2} \mathrm{Cl}_{2}, 8: 1: 1\right)$ to afford 5 (397 $\mathrm{mg}, 96 \%$ ) as a white foam. $[\alpha]^{24}=-7.4\left(c\right.$ 1.0, $\left.\mathrm{CHCl}_{3}\right) ;{ }^{1} \mathrm{H}$ NMR (300 MHz, $\left.\mathrm{CDCl}_{3}\right): \delta$ 7.69-7.33 (m, 10 H), 5.13 (m, 1 H), 4.36 (m, 1 H), 3.57-3.48 (m, 1 H), 1.06 (s, 9 H), 1.01 (s, 3 H), 0.95 (s, $3 \mathrm{H}) ;{ }^{13} \mathrm{C}$ NMR (75 MHz, $\left.\mathrm{CDCl}_{3}\right): \delta 219.4,141.4,135.7,134.7,129.4,127.4,120.3,73.0$, 71.2, 50.0, 48.5, 47.4, 42.4, 37.0, 36.5, 31.7, 31.4, 31.1, 30.5, 30.3, 27.0, 19.8, 19.4, 19.1, 13.8; ESIMS m/z: $560.27\left(\mathrm{M}+\mathrm{Na}^{+}\right)$; Anal. Calcd for $\mathrm{C}_{35} \mathrm{H}_{46} \mathrm{O}_{3} \mathrm{Si}$ : C, 77.44; $\mathrm{H}, 8.54$; Found: C, 77.04; H, 8.47.

\section{6 $\alpha$-(tert-Butyldimethylsiloxy)-3 $\beta$-(tert-butyldiphenylsiloxy)-5-androsten-17-one (7)}

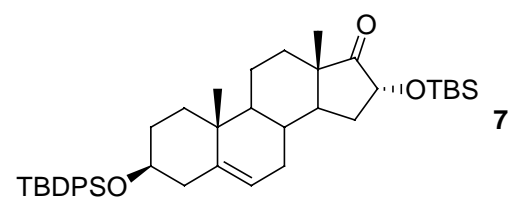

To a solution of $5(2.4 \mathrm{~g})$ and imidazole $(780 \mathrm{mg})$ in dry $\mathrm{CH}_{2} \mathrm{Cl}_{2}(10 \mathrm{~mL})$ was added tert-butyldimethylsilyl chloride (833 mg) at rt. After stirring overnight, the suspension was diluted with $\mathrm{CH}_{2} \mathrm{Cl}_{2}$. The organic layer was washed with saturated $\mathrm{NaHCO}_{3}$ and brine, respectively, and then dried over $\mathrm{Na}_{2} \mathrm{SO}_{4}$, and filtered. The filtrate was concentrated in vacuo to give a residue, which was purified by a flash column chromatography (petroleum ether:EtOAc, 90:1) to afford 7 (2.53 g, 86\%) as a white foam. $[\alpha]^{24}=-3.7\left(c\right.$ 1.0, $\left.\mathrm{CHCl}_{3}\right) ;{ }^{1} \mathrm{H}$ NMR (300 MHz, $\left.\mathrm{CDCl}_{3}\right): \delta$ 7.69-7.38 (m, $\left.10 \mathrm{H}\right), 5.16(\mathrm{~m}$, $1 \mathrm{H}$ ), 4.33 (d, $J=7.4 \mathrm{~Hz}, 1 \mathrm{H}), 3.59-3.51$ (m, 1 H), 1.08 (s, 9 H), 1.02 (s, 3 H), 0.9 (s, 9 H), 0.92 (s, $3 \mathrm{H}$ ), 0.13 (s, $3 \mathrm{H}$ ), 0.12 (s, $3 \mathrm{H}$ ); ${ }^{13} \mathrm{C}$ NMR (75 MHz,CDCl 3 ): $\delta$ 217.8, 141.4, 135.7, 134.7, 129.5, 127.5, 73.1, 72.3, 50.0, 48.6, 47.0, 42.4, 37.0, 36.6, 32.8, 31.8, 31.4, 31.3, 30.5, 27.0, 25.8, 20.0, 19.4, 19.1, 18.4, 14.3, -4.6, -5.2; EIMS m/z (\%): $599\left(\mathrm{M}^{+}-57\right.$, 30.9), 343 (67.8), 199 (100).

\section{Typical procedure for aldol reaction under Conditions A}


Diisopropylamine $(1.7 \mathrm{~mL}, 11.7 \mathrm{mmol})$ was cooled to $-78{ }^{\circ} \mathrm{C}$, and a solution of n-butyllithium in hexanes (1.6 M, 7.3 mL) was added slowly by syringe under Ar. This mixture was stirred for $15 \mathrm{~min}$ at $-78{ }^{\circ} \mathrm{C}$, and then the ice bath was removed and the flask was placed under reduced pressure to remove the hexanes and the excess of $i-\mathrm{Pr}_{2} \mathrm{NH}$ to afford LDA as a white solid. After the flask was filled with Ar again, dry THF and HMPA (30 mL, v/v, 4:1) were added quickly, and the solution was cooled to $-78{ }^{\circ} \mathrm{C}$ immediately. Then a solution of ethyl propionate $(1.38 \mathrm{~mL})$ in dry THF $(5 \mathrm{~mL})$ was added dropwise. After stirring for $30 \mathrm{~min}$, a solution of ketone 6 (610 mg, $1.46 \mathrm{mmol})$ in THF (2 mL) was added. The resulting solution was stirred at $-78{ }^{\circ} \mathrm{C}$ for about $5 \mathrm{~h}$. The reaction mixture was then poured into 5\% $\mathrm{HCl}$, and extracted with EtOAc. The organic layer was washed with saturated $\mathrm{NaHCO}_{3}$ and brine and dried over $\mathrm{Na}_{2} \mathrm{SO}_{4}$. After evaporation of the solvent, the residue was purified by a flash column chromatography (petroleum ether:EtOAc, 18:1-15:1) to afford 9a (481 mg, 63\%) as a white solid.

(20S)-Ethyl 3 $\beta$-(tert-butyldiphenylsiloxy)-16 $\alpha, 17 \alpha$-dihydroxy-20-methyl-22-oate (8a)

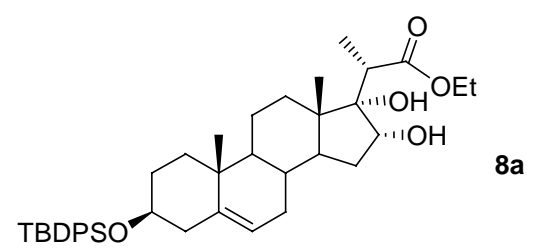

Compound 8a (1.08 g, 41\%) was obtained as a white solid. [ $\alpha]^{24}=-51.3\left(c\right.$ 1.2, $\left.\mathrm{CHCl}_{3}\right)$; ${ }^{1} \mathrm{H}$ NMR (300 MHz, CDCl $)$ ): $\delta$ 7.70-7.33 (m, $\left.10 \mathrm{H}\right), 5.12$ (m, $\left.1 \mathrm{H}\right), 4.18$ (m, $\left.1 \mathrm{H}\right), 4.15$ (q, $J=7.1 \mathrm{~Hz}, 2 \mathrm{H}), 3.53$ (m, 1 H), 3.53 (s, 1 H), 2.78 (q, $J=6.6 \mathrm{~Hz}, 1 \mathrm{H}), 2.75$ (br, $1 \mathrm{H}$ ), 1.28 (t, $J=7.1 \mathrm{~Hz}, 3 \mathrm{H}), 1.26$ (d, $J=6.6 \mathrm{~Hz}, 3 \mathrm{H}), 1.07$ (s, $9 \mathrm{H}), 0.98$ (s, $3 \mathrm{H}), 0.79$ (s, 3 $\mathrm{H}) ;{ }^{13} \mathrm{C}$ NMR (75 MHz, $\left.\mathrm{CDCl}_{3}\right): \delta 177.1,141.2,135.8,134.8,129.4,127.4,120.8,81.7$, 76.4, 73.2, 61.0, 49.3, 48.4, 48.1, 44.7, 42.4, 37.0, 36.4, 35.0, 32.3, 31.8, 31.7, 29.7, 27.0, 20.2, 19.3, 19.1, 14.6, 14.0, 12.7; ESIMS m/z: $645.3\left(\mathrm{M}^{+}+1\right), 667.3\left(\mathrm{M}+\mathrm{Na}^{+}\right)$; HRMS (MALDI) $\mathrm{m} / \mathrm{z}: 667.3798\left(\mathrm{M}+\mathrm{Na}^{+}\right)$; Calcd for $\mathrm{C}_{40} \mathrm{H}_{56} \mathrm{O}_{5} \mathrm{SiNa}$ : 667.3789.

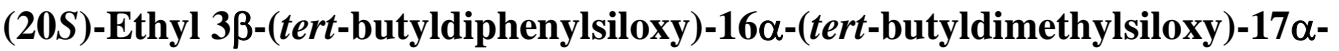
hydroxy-20-methyl-22-oate (10a) 


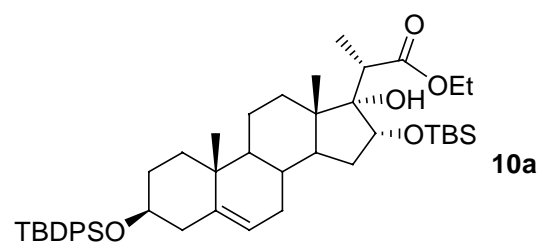

Compound 10a (1.48 g, 47\%) was obtained as a white solid. $[\alpha]_{\mathrm{D}}^{24}=-3.7\left(c\right.$ 1.0, $\left.\mathrm{CHCl}_{3}\right)$; ${ }^{1} \mathrm{H}$ NMR (300 MHz, $\mathrm{CDCl}_{3}$ ): $\delta$ 7.69-7.33 (m, $10 \mathrm{H}$ ), 5.09 (m, $\left.1 \mathrm{H}\right), 4.12$ (q, $J=7.2 \mathrm{~Hz}, 2$ H), 4.12 (m, 1 H), 3.60 (br, 1 H), 3.50 (m, 1 H), 2.66 (q, $J=7.2$ Hz, 1 H), 1.26 (t, $J=7.2$ Hz, 3 H), 1.15 (d, J = 7.2 Hz, 3 H), 1.05 (s, 9 H), 0.96 (s, 3 H), 0.89 (s, 9 H), 0.83 (s, 3 H), 0.09 (s, $6 \mathrm{H}) ;{ }^{13} \mathrm{C}$ NMR (75 MHz, $\left.\mathrm{CDCl}_{3}\right): \delta 174.6,141.4,135.8,134.8,129.4,127.4$, 120.7, 81.9, 73.2, 60.0, 49.4, 48.7, 47.3, 44.4, 42.5, 37.1, 36.4, 35.5, 31.8, 31.7, 31.5, 30.4, 27.0, 25.8, 20.1, 19.4, 19.1, 17.8, 14.5, 14.1, 13.2, -3.5, -4.8; ESIMS m/z: $759.3\left(\mathrm{M}+\mathrm{H}^{+}\right)$, $781.3\left(\mathrm{M}+\mathrm{Na}^{+}\right)$; Anal. Calcd for $\mathrm{C}_{46} \mathrm{H}_{70} \mathrm{O}_{5} \mathrm{Si}_{2}$ : C, 72.77; H, 9.29; Found: C, 72.41; $\mathrm{H}$, 9.30 .

\section{Typical procedure for aldol reaction under Conditions B}

A similar procedure as described for Conditions A was used, except without removal of the hexane and the excess of $i-\mathrm{Pr}_{2} \mathrm{NH}$ under reduced pressure in the preparation of LDA.

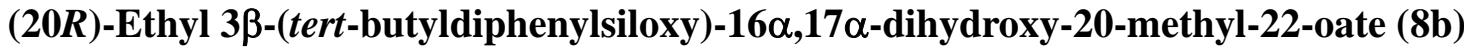

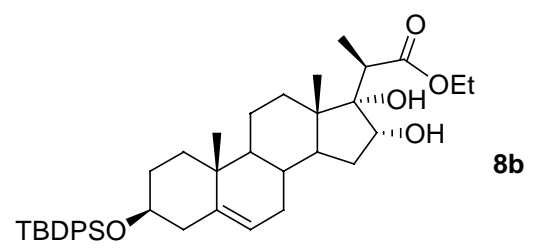

Compound 8b (174 mg, 29\%) was obtained as a white solid, besides 8a (243 mg, 41\%). 8b: $[\alpha]^{24}{ }_{\mathrm{D}}=-56.7\left(c\right.$ 1.3, $\left.\mathrm{CHCl}_{3}\right) ;{ }^{1} \mathrm{H}$ NMR (300 MHz, $\left.\mathrm{CDCl}_{3}\right): \delta 7.70-7.34(\mathrm{~m}, 10 \mathrm{H})$, 5.12 (m, 1 H), 4.38 (br, 1 H), 4.16 (dq, $J=2.5,7.1$ Hz, 2 H), 4.00 (m, 1 H), 3.57-3.46 (m, $1 \mathrm{H}), 3.49$ (br, $1 \mathrm{H}$ ), 2.74 (q, $J=7.1 \mathrm{~Hz}, 1 \mathrm{H}), 1.29$ (t, $J=7.1 \mathrm{~Hz}, 3 \mathrm{H}), 1.30$ (d, $J=7.1$ Hz, $3 \mathrm{H}$ ), 1.06 (s, $9 \mathrm{H}), 0.97$ (s, $3 \mathrm{H}), 0.75$ (s, $3 \mathrm{H}$ ); ${ }^{13} \mathrm{C}$ NMR (75 MHz, $\mathrm{CDCl}_{3}$ ): $\delta$ 177.5, 141.0, 135.7, 134.8, 134.7, 129.4, 129.4, 127.4, 127.4, 121.0, 82.3, 75.3, 73.1, 60.9, 49.3, 48.0, 47.5, 42.4, 41.4, 37.1, 36.4, 36.1, 31.8, 31.7, 31.6, 31.5, 27.0, 20.2, 19.3, 19.1, 15.3, 
14.0, 13.9; IR (KBr): 3464, 3072, 2960, 2933, 1708, 1590, 1186, 702, $508 \mathrm{~cm}^{-1}$; ESIMS m/z: $645.3\left(\mathrm{M}+\mathrm{H}^{+}\right), 667.3\left(\mathrm{M}+\mathrm{Na}^{+}\right)$; Anal. Calcd for $\mathrm{C}_{40} \mathrm{H}_{56} \mathrm{O}_{5} \mathrm{Si}: \mathrm{C}, 74.49 ; \mathrm{H}, 8.75$; Found: C, 74.11; H, 8.86.

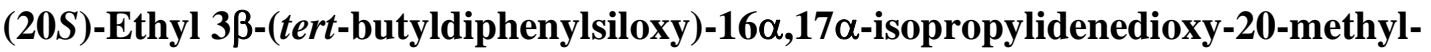

\section{2-oate (13)}

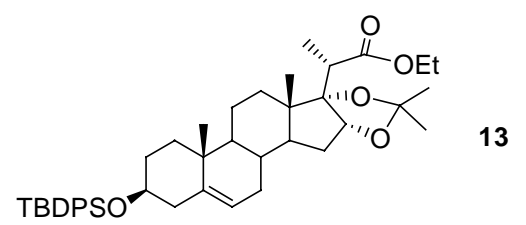

Diol 8a (50 mg, $0.08 \mathrm{mmol})$ and 2,2-dimethoxypropane $(0.05 \mathrm{~mL})$ in acetone $(1 \mathrm{~mL})$ were treated with cat. TsOH $\bullet \mathrm{H}_{2} \mathrm{O}$ at rt for $30 \mathrm{~min}$. The reaction was quenched with $\mathrm{Et}_{3} \mathrm{~N}$ and then concentrated in vacuo to give a residue, which was purified by a flash column chromatography (petroleum ether:EtOAc, 12:1) to afford 13 (49 mg, 92\%) as a white foam. $[\alpha]^{24}{ }_{\mathrm{D}}=-21.5$ (c 1.0, $\left.\mathrm{CHCl}_{3}\right) ;{ }^{1} \mathrm{H}$ NMR (300 MHz, $\left.\mathrm{CDCl}_{3}\right): \delta 7.71(\mathrm{~m}, 10 \mathrm{H}), 5.12$ (m, $1 \mathrm{H}), 4.87$ (d, $J=7.2 \mathrm{~Hz}, 1 \mathrm{H}), 4.21-4.08$ (m, $2 \mathrm{H}), 3.55-3.48$ (m, $1 \mathrm{H}), 2.92$ (q, $J=$ $7.0 \mathrm{~Hz}, 1 \mathrm{H}), 1.40$ (s, $3 \mathrm{H}), 1.38$ (s, $3 \mathrm{H}), 1.27$ (t, $J=7.2 \mathrm{~Hz}), 1.21$ (d, $J=7.0 \mathrm{~Hz}, 3 \mathrm{H})$, 1.06 (s, $9 \mathrm{H}), 0.99$ (s, $3 \mathrm{H}), 0.83$ (s, $3 \mathrm{H}), 0.07$ (s, $6 \mathrm{H}) ;{ }^{13} \mathrm{C} \mathrm{NMR}\left(75 \mathrm{MHz}, \mathrm{CDCl}_{3}\right): \delta$ 175.0, 141.5, 135.7, 134.8, 129.4, 127.4, 120.7, 111.9, 96.3, 83.6, 73.2, 60.5, 49.8, 49.3, 48.1, 42.5, 42.3, 37.1, 36.5, 34.1, 33.0, 32.0, 31.8, 31.8, 28.9, 28.5, 27.0, 20.4, 19.4, 19.1, 17.0, 14.2, 13.0; ESIMS m/z: $707.4\left(\mathrm{M}+\mathrm{Na}^{+}\right)$; Anal. Calcd for $\mathrm{C}_{43} \mathrm{H}_{60} \mathrm{O}_{5} \mathrm{Si}: \mathrm{C}, 75.39 ; \mathrm{H}$, 8.83; Found: C, 75.04; H, 8.46.

\section{Weinreb amide 14}

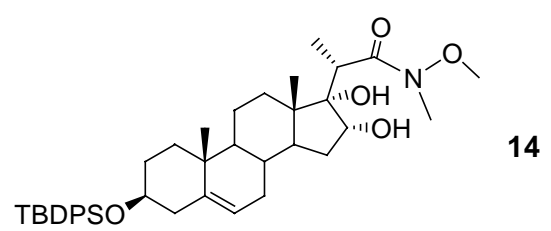

Ester 8a (1.7 g, $2.64 \mathrm{mmol})$ and N,O-dimethylhydroxylamine hydrochloride (2.6 g, 26.4 mmol) were suspended in THF. The suspension was cooled to $-10{ }^{\circ} \mathrm{C}$, and treated with i-PrMgCl (30 mL of $2 \mathrm{M}$ in THF) over 15 min to create a homogeneous mixture. After 
stirring at $0^{\circ} \mathrm{C}$ for an additional $4 \mathrm{~h}$, the reaction was quenched by addition of a saturated ammonium chloride aqueous solution. The product was extracted with ethyl acetate, and the combined organic layers were dried over $\mathrm{Na}_{2} \mathrm{SO}_{4}$. After evaporation of the solvent, the residue was purified by a flash column chromatography (petroleum ether:EtOAc, 1.5:1) to afford 14 (1.30 g, 75\%) as a white solid and to recover 8a (0.63 g, 7\%). 14: $[\alpha]^{24}{ }_{\mathrm{D}}=-36.6$ (c 0.8, $\left.\mathrm{CHCl}_{3}\right) ;{ }^{1} \mathrm{H}$ NMR (300 MHz, $\mathrm{CDCl}_{3}$ ): $\delta$ 7.70-7.34 (m, $10 \mathrm{H}$ ), 5.12 (m, 1 H), 4.67 (br, 1 H), 4.00 (m, 1 H), 3.72 (s, 3 H), 3.59-3.50 (m, 1 H), 3.31 (q, $J=6.6$ Hz, 1 H), 3.19 (s, 3 H), 2.57 (br, 1 H), 1.23 (d, J = 6.6 Hz, 3 H), 1.06 (s, 9 H), 0.99 (s, 3 $\mathrm{H}), 0.81$ (s, $3 \mathrm{H}) ;{ }^{13} \mathrm{C}$ NMR (75 MHz, $\left.\mathrm{CDCl}_{3}\right): \delta$ 178.1, 141.1, 135.7, 134.8, 129.4, 127.4, 120.9, 82.5, 73.1, 61.7, 49.3, 48.2, 48.0, 42.4, 37.5, 37.0, 36.4, 35.6, 32.3, 32.2, 31.8, 31.7, 31.7, 27.0, 20.3, 19.4, 19.1, 14.6, 13.4; ESIMS m/z: $660.3\left(\mathrm{M}+\mathrm{H}^{+}\right), 682.2\left(\mathrm{M}+\mathrm{Na}^{+}\right)$; HRMS (ESI) m/z: $682.3890\left(\mathrm{M}+\mathrm{Na}^{+}\right)$; Calcd for $\mathrm{C}_{40} \mathrm{H}_{57} \mathrm{O}_{5} \mathrm{SiNNa}$ : 682.3898 .

\section{Methyl ketone 15}

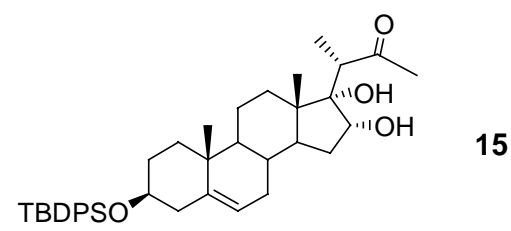

Compound 15 was obtained as a white solid (51\%) using a similar procedure as described for the preparation of 17. $[\alpha]^{24}=-53.1\left(c\right.$ 1.0, $\left.\mathrm{CHCl}_{3}\right)$; ${ }^{1} \mathrm{H}$ NMR $\left(300 \mathrm{MHz}, \mathrm{CDCl}_{3}\right)$ : $\delta$ 7.68-7.34 (m, 10 H), 5.10 (m, 1 H), 4.09 (m, 1 H), 3.77 (br, 1 H), 3.58-3.48 (m, 1 H), 2.87 (q, $J=6.9$ Hz, 1 H), 2.72 (br, 1 H), 2.24 (s, 3 H), 1.21 (d, $J=6.9$ Hz, 3 H), 1.05 (s, 9 H), 0.97 (s, $3 \mathrm{H}), 0.78$ (s, $3 \mathrm{H}) ;{ }^{13} \mathrm{C}$ NMR $\left(75 \mathrm{MHz}, \mathrm{CDCl}_{3}\right): \delta 216.3,141.2,135.7,134.8$, 129.4, 127.4, 120.8, 82.4, 73.1, 71.2, 50.7, 50.0, 49.3, 48.5, 48.4, 48.2, 42.4, 37.0, 36.4, 35.0, 32.3, 31.8, 31.7, 31.4, 31.2, 30.7, 30.6, 30.3, 27.0, 20.2, 19.9, 19.3, 19.1, 14.6, 13.8, 12.4; ESIMS m/z: $637.3\left(\mathrm{M}+\mathrm{Na}^{+}\right)$; Anal. Calcd for $\mathrm{C}_{39} \mathrm{H}_{54} \mathrm{O}_{4} \mathrm{Si}$ : C, 76.17; H, 8.85; Found: C, 76.11; H, 9.25.

\section{Butyl ketone 16}




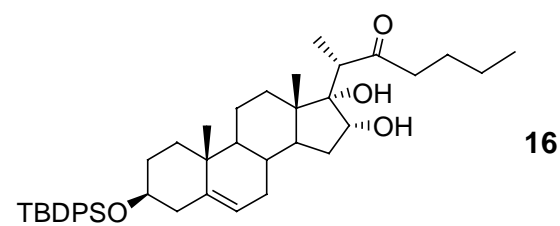

Compounds 16 (12\%), 18 (27\%), and 19 (38\%) were obtained as white solids using a similar procedure as described for the preparation of 17. Compound 16: $[\alpha]^{24}=-38.8(c$ 1.0, $\mathrm{CHCl}_{3}$ ); ${ }^{1} \mathrm{H}$ NMR (300 MHz, $\mathrm{CDCl}_{3}$ ): $\delta$ 7.69-7.34 (m, $10 \mathrm{H}$ ), 5.10 (m, $\left.1 \mathrm{H}\right), 4.07$ (br, 1 H), 4.05-3.97 (m, 1 H), 3.53 (m, 1 H), 2.89 (q, $J=7.1 \mathrm{~Hz}, 1 \mathrm{H}), 2.55$ (t, $J=8.3 \mathrm{~Hz}, 2$ H), 2.55 (br, $1 \mathrm{H}), 1.17$ (d, J = 7.1 Hz, $3 \mathrm{H}), 1.06$ (s, $9 \mathrm{H}), 0.98$ (s, $3 \mathrm{H}), 0.78$ (s, $3 \mathrm{H}) ;{ }^{13} \mathrm{C}$ NMR (75 MHz, $\left.\mathrm{CDCl}_{3}\right): \delta$ 218.3, 141.2, 135.8, 134.8, 129.4, 127.4, 120.9, 82.6, 76.9, 73.1, 72.8, 49.3, 48.2, 43.4, 42.4, 41.6, 37.0, 36.4, 35.2, 31.8, 31.7, 27.0, 26.1, 25.4, 23.3, 22.2, 20.2, 19.4, 19.1, 14.5, 14.1, 13.8, 12.6; ESIMS m/z: $657.4\left(\mathrm{M}+\mathrm{H}^{+}\right), 679.4(\mathrm{M}+$ $\mathrm{Na}^{+}$); HRMS (ESI) m/z: $679.4153\left(\mathrm{M}+\mathrm{Na}^{+}\right)$; Calcd for $\mathrm{C}_{42} \mathrm{H}_{60} \mathrm{O}_{4} \mathrm{SiNa}$ : 679.4158.

\section{Compounds 17-19}
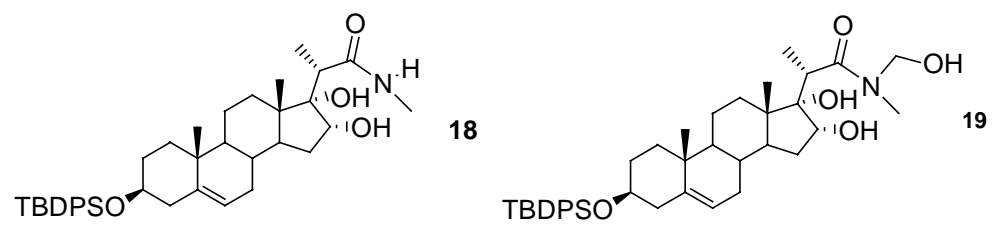

Amide 14 (300 mg) was dissolved in $\mathrm{Et}_{2} \mathrm{O}(25 \mathrm{~mL})$. The solution was cooled to $-20{ }^{\circ} \mathrm{C}$, and treated dropwise with isoamyl lithium (15 mL, 0.78 $\mathrm{M}$ in $\left.\mathrm{Et}_{2} \mathrm{O}\right)$. After stirring at -20 ${ }^{\circ} \mathrm{C}$ for an additional $1.5 \mathrm{~h}$, the resulting reaction mixture was pooled into $5 \% \mathrm{HCl}$ which has been cooled to $-40{ }^{\circ} \mathrm{C}$. The product was extracted with $\mathrm{CH}_{2} \mathrm{Cl}_{2}$, and the combined organic layers were dried over $\mathrm{Na}_{2} \mathrm{SO}_{4}$. After evaporation of the solvent, the residue was purified by a flash column chromatography (petroleum ether:EtOAc, 12:1) to afford $\mathbf{1 7}$ (112 mg, 40\%), 18 (65 mg, 22\%), and 19 (96 mg, 32\%) as white solids. The data of 17 have been given in the Experimental Section.

Compound 18: $[\alpha]^{24}=-70.1$ (c 1.3, $\left.\mathrm{CHCl}_{3}\right)$; ${ }^{1} \mathrm{H}$ NMR (400 MHz, $\left.\mathrm{CDCl}_{3}\right): \delta 7.68-7.33$ (m, 10 H), 5.89 (br, 1 H), 5.10 (m, 1 H), 4.21 (m, 1 H), 4.19 (br, 1 H), 3.54 (m, 1 H), 2.99 (br, $1 \mathrm{H}), 2.78$ (d, $J=4.8 \mathrm{~Hz}, 3 \mathrm{H}), 2.56$ (q, $J=7.0 \mathrm{~Hz}, 1 \mathrm{H}), 1.23$ (d, $J=7.0 \mathrm{~Hz}, 3 \mathrm{H}$ ), 1.06 (s, $9 \mathrm{H}), 0.97$ (s, $3 \mathrm{H}), 0.76$ (s, $\left.3 \mathrm{H}) ;{ }^{13} \mathrm{C} \mathrm{NMR} \mathrm{(100} \mathrm{MHz,} \mathrm{CDCl}_{3}\right): \delta$ 177.6, 141.1, 135.7, 134.8, 129.4, 127.4, 120.9, 81.9, 76.4, 73.2, 49.3, 48.3, 48.1, 46.2, 42.4, 37.0, 36.4, 
34.8, 32.3, 31.8, 31.7, 27.0, 26.4, 20.2, 19.3, 19.1, 14.4, 13.4; ESIMS m/z: 630.3 (M + $\mathrm{H}^{+}$); HRMS (ESI) m/z: $630.3983\left(\mathrm{M}+\mathrm{H}^{+}\right.$); Calcd for $\mathrm{C}_{39} \mathrm{H}_{56} \mathrm{O}_{4} \mathrm{SiN}: 630.3973$.

Compound 19: $[\alpha]^{24}=-60.5\left(c\right.$ 1.1, $\left.\mathrm{CHCl}_{3}\right)$; ${ }^{1} \mathrm{H}$ NMR $\left(400 \mathrm{MHz}, \mathrm{CDCl}_{3}\right): \delta$ 7.68-7.34 (m, 10 H), 6.71 (br , 1 H), 5.10 (m, 1 H), 4.46 (2 H), 4.26 (m, 1 H), 3.9 (br, 1 H), 3.54 (m, $1 \mathrm{H}$ ), 3.33 (s, 1 H), 3.09 (br, 1 H), 2.63 (q, $J=7.0 \mathrm{~Hz}, 1 \mathrm{H}$ ), 1.25 (d, $J=7.0 \mathrm{~Hz}, 3 \mathrm{H}$ ), 1.05 (s, $9 \mathrm{H}), 0.97$ (s, $3 \mathrm{H}), 0.77$ (s, $3 \mathrm{H}) ;{ }^{13} \mathrm{C}$ NMR (100 MHz, $\mathrm{CDCl}_{3}$ ): $\delta$ 177.6, 141.2, 135.8, 134.8, 129.4, 127.5, 120.8, 81.7, 75.8, 73.2, 71.5, 56.1, 49.4, 48.4, 46.8, 42.4, 37.1, 36.4, 34.7, 32.5, 31.8, 31.2, 22.3, 19.4, 19.1, 14.8, 13.0; ESIMS m/z: $682.2\left(\mathrm{M}^{+} \mathrm{Na}^{+}\right)$; HRMS (ESI) m/z: $682.3891\left(\mathrm{M}+\mathrm{Na}^{+}\right)$; Calcd for $\mathrm{C}_{40} \mathrm{H}_{57} \mathrm{O}_{5} \mathrm{SiNNa}$ : 682.3898 .

\section{2-Aldehyde 23}

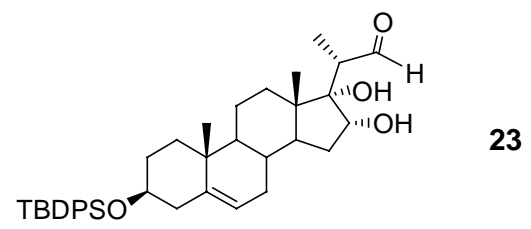

Amide 14 (410 mg) was dissolved in THF $(10 \mathrm{~mL})$. The solution was cooled to $-78{ }^{\circ} \mathrm{C}$ and treated dropwise with DIBAL-H (2.5 mL, 1.0 M in toluene). After stirring at $-78{ }^{\circ} \mathrm{C}$ for an additional $3 \mathrm{~h}$, the resulting reaction mixture was quenched with $\mathrm{MeOH}$. The product was extracted with $\mathrm{CH}_{2} \mathrm{Cl}_{2}$, and the combined organic layers were washed with brine and dried over $\mathrm{Na}_{2} \mathrm{SO}_{4}$. After evaporation of the solvent, the residue was purified by a flash column chromatography (petroleum ether:EtOAc, 12:1) to afford 23 (59 mg, 15\%) as a white solid and to recover $8 \mathbf{a}(255 \mathrm{mg}, 58 \%)$. $[\alpha]^{24}{ }_{\mathrm{D}}=-78.1\left(\mathrm{c} 0.6, \mathrm{CHCl}_{3}\right) ;{ }^{1} \mathrm{H} \mathrm{NMR}$ (300 MHz, $\left.\mathrm{CDCl}_{3}\right): \delta 9.59$ (s, $\left.1 \mathrm{H}\right)$, 7.67-7.33 (m, $\left.10 \mathrm{H}\right), 5.12(\mathrm{~m}, 1 \mathrm{H}), 4.29(\mathrm{~m}, 1 \mathrm{H})$, 3.53 (m, 1 H), 3.43 (br, 1 H), 3.16 (s, 1 H), 2.60 (q, $J=7.3$ Hz, 1 H), 1.35 (d, $J=7.3$ Hz, $3 \mathrm{H}), 1.06$ (s, $9 \mathrm{H}), 0.98$ (s, $3 \mathrm{H}), 0.77$ (s, $3 \mathrm{H}$ ); ${ }^{13} \mathrm{C}$ NMR (75 MHz, $\mathrm{CDCl}_{3}$ ): $\delta$ 208.2, 141.2, 135.7, 134.8, 129.4, 127.4, 120.8, 81.7, 76.0, 73.1, 52.5, 49.3, 48.6, 48.2, 42.4, 37.0, 36.4, 34.6, 32.4, 31.7, 31.6, 27.0, 20.2, 19.3, 19.1, 14.8, 9.6; ESIMS m/z: 623.4 (M $+\mathrm{Na}^{+}$); HRMS (ESI) m/z: $623.3527\left(\mathrm{M}+\mathrm{Na}^{+}\right)$; Calcd for $\mathrm{C}_{38} \mathrm{H}_{52} \mathrm{O}_{4} \mathrm{SiNa}$ : 623.3538.

\section{Thioacetal 24}




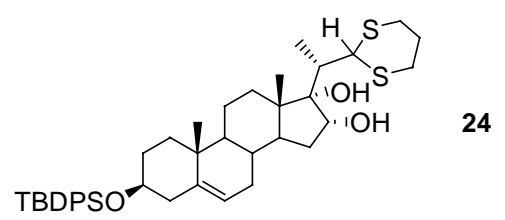

Aldehyde 23 (8 mg) and $\mathrm{I}_{2}$ (1 mg, cat.) were dissolved in THF (1.5 mL), then 1,3-propanedithiol was added. The resulting reaction mixture was concentrated in vacuo after being stirred at room temperature for $0.5 \mathrm{~h}$. The residue was purified by a flash column chromatography (petroleum ether:EtOAc, 8:1) to afford 24 (8 $\mathrm{mg}, 87 \%$ ) as a white solid. $[\alpha]^{24}=-34.8$ (c 1.2, $\mathrm{CHCl}_{3}$ ); ${ }^{1} \mathrm{H}$ NMR (300 MHz, $\mathrm{CDCl}_{3}$ ): $\delta$ 7.69-7.34 (m, $10 \mathrm{H}), 5.10$ (m, $1 \mathrm{H}), 4.69$ (s, $1 \mathrm{H}), 4.17$ (br, $1 \mathrm{H})$, 3.58-3.48 (m, $1 \mathrm{H}), 3.22(\mathrm{~s}, 1 \mathrm{H})$, 3.07-2.77 (5 H), 1.22 (d, $J=7.1 \mathrm{~Hz}, 3 \mathrm{H}), 1.05$ (s, $9 \mathrm{H}), 0.96$ (s, $3 \mathrm{H}), 0.74$ (s, $3 \mathrm{H}) ;{ }^{13} \mathrm{C}$ NMR (75 MHz, $\left.\mathrm{CDCl}_{3}\right): \delta 141.3,135.8,134.8,129.4,127.4,120.8,85.4,73.2,53.7,49.3$, 48.3, 48.1, 44.6, 42.4, 37.0, 36.4, 36.0, 32.2, 31.0, 31.7, 31.1, 27.0, 25.9, 20.3, 19.3, 19.1, 14.7, 12.2; ESIMS m/z (\%): $713.4\left(\mathrm{M}+\mathrm{Na}^{+}\right)$; HRMS (ESI) $\mathrm{m} / \mathrm{z}: 713.3489\left(\mathrm{M}+\mathrm{Na}^{+}\right)$; Calcd for $\mathrm{C}_{41} \mathrm{H}_{58} \mathrm{O}_{3} \mathrm{~S}_{2} \mathrm{SiNa}$ : 713.3522 .

\section{5,16,20(22)-Triene 29}

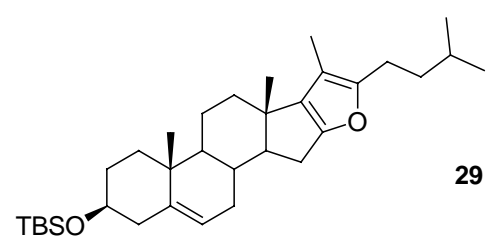

To a solution of lactone 26 (190 mg, $0.40 \mathrm{mmol})$ in THF (5 mL) was added a solution of isoamyl lithium in $\mathrm{Et}_{2} \mathrm{O}(1.35 \mathrm{M}, 1.78 \mathrm{~mL}, 2.40 \mathrm{mmol})$ dropwise during $0.5 \mathrm{~h}$ at room temperature under argon. After continuous strirring for another $30 \mathrm{~min}, \mathrm{LiAlH}_{4}(30 \mathrm{mg}$, $0.80 \mathrm{mmol})$ in THF (2 mL) was added. The solution was stirred at $0{ }^{\circ} \mathrm{C}$ overnight. The reaction mixture was quenched with $\mathrm{MeOH}$, and extracted with $\mathrm{CH}_{2} \mathrm{Cl}_{2}$. The organic layer was washed with saturated $\mathrm{NH}_{4} \mathrm{Cl}$ and brine and dried over $\mathrm{Na}_{2} \mathrm{SO}_{4}$. After evaporation of the solvent, the residue was purified by a flash column chromatography (petroleum ether:EtOAc: $\left.\mathrm{CH}_{2} \mathrm{Cl}_{2}, 6: 1: 1\right)$ to afford 29 (157 mg, 77\%) and 30 (25 mg, 12\%) as white solids. Compound 29: ${ }^{1} \mathrm{H}$ NMR (300 MHz, $\mathrm{CDCl}_{3}$ ): $\delta 5.35$ (m, $1 \mathrm{H}$ ), 3.49 (m, 1 H), 1.90 (s, 3 H), 1.13 (s, 3 H), 0.91 (s, 3 H), 0.91 (d, 6 H), 0.89 (s, 9 H), 0.06 (s, 6 H); ${ }^{13} \mathrm{C}$ NMR (75 MHz, $\left.\mathrm{CDCl}_{3}\right): \delta 155.0,153.8,142.0,136.8,120.9,111.4,72.7,60.7,51.0$, 
43.0, 41.2, 38.1, 37.3, 37.1, 35.6, 32.2, 32.1, 31.8, 30.4, 28.4, 27.8, 26.1, 24.6, 22.6, 22.5, 20.6, 19.5, 18.1, 9.0, -4.5; ESIMS m/z: $529.4\left(\mathrm{M}+\mathrm{H}_{2} \mathrm{O}+\mathrm{H}^{+}\right)$; HRMS (ESI) m/z: $511.3954\left(\mathrm{M}+\mathrm{H}^{+}\right)$; Calcd for $\mathrm{C}_{33} \mathrm{H}_{55} \mathrm{O}_{2} \mathrm{Si}$ : 511.3966.

\section{Typical procedure for aldol reaction under Conditions C}

A solution of $i-\mathrm{Pr}_{2} \mathrm{NH}(4.4 \mathrm{~mL}, 30 \mathrm{mmol})$ in dry THF $(30 \mathrm{~mL})$ was cooled to $-78{ }^{\circ} \mathrm{C}$, and a solution of $n$-butyllithium in hexanes $(1.6 \mathrm{M}, 18.5 \mathrm{~mL})$ was added slowly by syringe under Ar. After stirring for $15 \mathrm{~min}$ at $-78^{\circ} \mathrm{C}$, a solution of ethyl propionate $(3.5 \mathrm{~mL}, 30$ mmol) in THF (5 mL) was added dropwise. After continuous stirring for $30 \mathrm{~min}$, a solution of ketone 6 (2.07 g, $4.94 \mathrm{mmol})$ in THF (10 mL) was added. The solution was stirred at $-78{ }^{\circ} \mathrm{C}$ for about $5 \mathrm{~h}$. The reaction mixture was poured into $5 \% \mathrm{HCl}$, and extracted with EtOAc. The organic layer was washed with saturated $\mathrm{NaHCO}_{3}$ and brine and dried over $\mathrm{Na}_{2} \mathrm{SO}_{4}$. After evaporation of the solvent, the residue was purified by a flash column chromatography (petroleum ether:EtOAc, 18:1-15:1) to afford 9a (1.94 g, 75\%) and $\mathbf{9 b}$ (308 mg, 12\%) as white solids.

(20S)-Allyl 3ß-(tert-butyldimethylsiloxy)-16 $\alpha, 17 \alpha$-dihydroxy-20-methyl-22-oate (41)

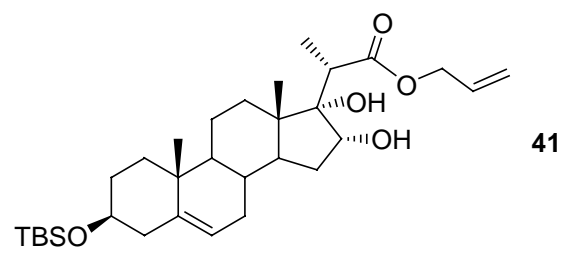

Compound 41 was obtained as a white solid. ${ }^{1} \mathrm{H}$ NMR (300 MHz, $\left.\mathrm{CDCl}_{3}\right): \delta$ 5.99-5.82 (m, 1 H), 5.37-5.23 (m, 4 H), 4.61-4.58 (m, 2 H), 3.45 (br, 1 H), 3.44-3.38 (m, $1 \mathrm{H})$, 2.82-2.72 (m, 2 H), 0.95 (s, 3 H), 0.84 (s, 3 H), 0.06 (s, 6 H); ESIMS m/z: 555.35 (M + $\left.\mathrm{Na}^{+}\right)$.

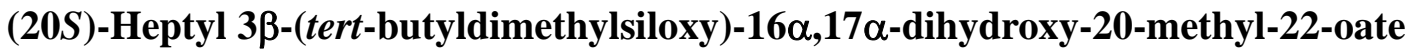
(42) 


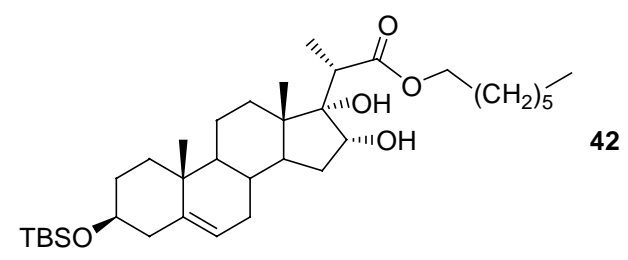

Compound 42 was obtained as a white solid. $[\alpha]^{13}=-36.9\left(c\right.$ 1.4, $\left.\mathrm{CHCl}_{3}\right) ;{ }^{1} \mathrm{H}$ NMR (300 MHz, $\mathrm{CDCl}_{3}$ ): $\delta 5.24$ (m, $\left.1 \mathrm{H}\right), 4.10-4.00$ (m, $\left.3 \mathrm{H}\right), 3.50$ (br, $\left.1 \mathrm{H}\right), 2.74$ (m, $\left.1 \mathrm{H}\right), 2.76$ (br, $1 \mathrm{H}), 2.73$ (q, $J=7.2 \mathrm{~Hz}, 1 \mathrm{H}$ ), 0.98 (s, $3 \mathrm{H}$ ), 0.88 (s. $3 \mathrm{H}), 0.80$ (s, $3 \mathrm{H}$ ), 0.05 (s, $6 \mathrm{H}$ ); ${ }^{13} \mathrm{C}$ NMR (75 MHz, $\left.\mathrm{CDCl}_{3}\right): \delta 177.2,141.4,120.8,81.7,76.4,72.5,65.2,49.5,48.4,8.1$, 44.8, 42.7, 37.2, 36.4, 35.0, 32.4, 32.0, 31.7, 31.6, 28.8, 28.4, 25.9, 25.8, 22.5, 20.3, 19.3, 18.2, 14.6, 14.0, 12.7, -4.5; ESIMS m/z: $613.75\left(\mathrm{M}+\mathrm{Na}^{+}\right)$; HRMS (ESI) m/z: 613.4273 $\left(\mathrm{M}+\mathrm{Na}^{+}\right)$; Calcd for $\mathrm{C}_{35} \mathrm{H}_{62} \mathrm{O}_{5} \mathrm{SiNa}$ : 613.4258 .

\section{(20S)-Octadecyl 3 $\beta$-(tert-butyldimethylsiloxy)-16 $\alpha, 17 \alpha$-dihydroxy-20-methyl-}

\section{2-oate (43)}

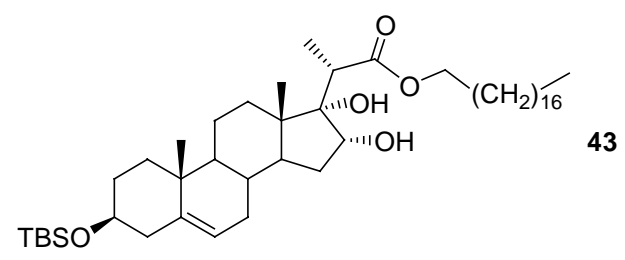

Compound 43 was obtained as a white solid. ${ }^{1} \mathrm{H}$ NMR (300 MHz, $\left.\mathrm{CDCl}_{3}\right): \delta 5.30$ (m, 1 H), 4.16 (m, 1 H), 4.11-4.03 (m, 2 H), 3.56 (br, 1 H), 3.51-3.44 (m, 1 H), 2.78 (m, 2 H), 0.95 (s, $3 \mathrm{H}), 0.88$ (s, $9 \mathrm{H}), 0.80$ (s, $3 \mathrm{H}), 0.06$ (s, $6 \mathrm{H})$; ESIMS m/z: $767.8\left(\mathrm{M}^{+} \mathrm{Na}^{+}\right)$; HRMS (ESI) m/z: $767.5950\left(\mathrm{M}+\mathrm{Na}^{+}\right.$); Calcd for $\mathrm{C}_{46} \mathrm{H}_{84} \mathrm{O}_{5} \mathrm{SiNa}$ : 767.5980.

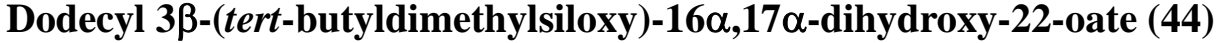

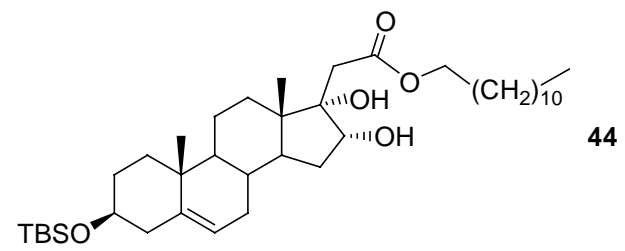

Compound 44 was obtained as a white solid. ${ }^{1} \mathrm{H}$ NMR (300 MHz, $\left.\mathrm{CDCl}_{3}\right): \delta 5.30(\mathrm{~m}, 1$ H), 4.33 (m, $1 \mathrm{H}), 4.07$ (t, $J=6.9 \mathrm{~Hz}, 2 \mathrm{H}), 3.87$ (d, $J=4.2 \mathrm{~Hz}, 1 \mathrm{H}$ ), 3.73 (br, $1 \mathrm{H}$ ), 3.56-3.42 (m, 1 H), 2.70 and 3.42 (AB, 2 H), 1.00 (s, 3 H), 0.70 (s, 3 H), 0.06 (s, 6 H). 
(20S)-Isobutyl 3 $\beta$-(tert-butyldimethylsiloxy)-16 $\alpha, 17 \alpha$-dihydroxy-20-methyl-22thioate (45)

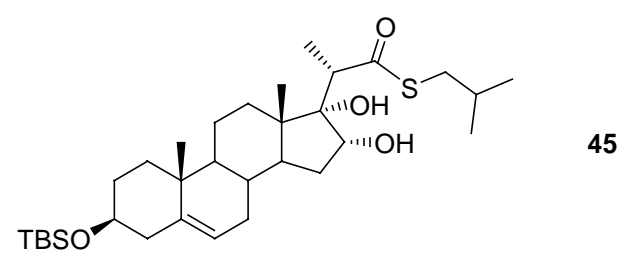

Compound 45 was obtained as a white solid (64\%). $[\alpha]^{17}{ }_{\mathrm{D}}=-11.0\left(\mathrm{c} 0.8, \mathrm{CHCl}_{3}\right) ;{ }^{1} \mathrm{H}$ NMR (300 MHz, $\left.\mathrm{CDCl}_{3}\right): \delta 8.01$ and $6.92(\mathrm{AB}, 4 \mathrm{H}), 5.30$ (m, $\left.1 \mathrm{H}\right), 5.00$ (m, $\left.1 \mathrm{H}\right), 4.85$ (m, 1 H), 4.23 (m, 1 H), 3.64 (br, 1 H), 3.51-3.44 (m, 1 H), 2.95 (q, J = 6.9 Hz, 1 H), 2.79 (m, 2 H), 1.30 (d, J = 6.9 Hz, 3 H), 0.98 (s, 3 H), 0.89 (s, 9 H), 0.81 (s, 3 H), 0.06 (s, 6 $\mathrm{H}) ;{ }^{13} \mathrm{C}$ NMR (75 MHz, $\left.\mathrm{CDCl}_{3}\right): \delta 205.5,141.4,120.8,82.4,76.2,72.5,52.9,49.4,48.4$, 48.2, 42.7, 37.4, 37.2, 36.5, 35.3, 32.3, 32.0, 31.7, 28.5, 25.9, 21.8, 21.7, 20.3, 19.4, 18.2, 14.8, 13.6, -4.6; ESIMS m/z: $587.5\left(\mathrm{M}+\mathrm{Na}^{+}\right)$; HRMS (ESI) m/z: $587.3567\left(\mathrm{M}+\mathrm{Na}^{+}\right)$; Calcd for $\mathrm{C}_{32} \mathrm{H}_{56} \mathrm{O}_{4} \mathrm{SSiNa}$ : 587.3561.

\section{General procedure for preparation of 16-ketones 47-48 and 50-52}

Tetrapropylammonium perruthenate $(0.2 \sim 0.26$ eq), $N$-methylmorpholine $N$-oxide (3 eq), and $4 \AA$ molecular sieves were added to a solution of the $16 \alpha, 17 \alpha$-dihydroxy compound (such as 9a, $1 \mathrm{eq}$ ) in $\mathrm{CH}_{2} \mathrm{Cl}_{2}$. The reaction mixture was stirred for $4 \mathrm{~h}$ before being diluted with $\mathrm{CH}_{2} \mathrm{Cl}_{2}$, filtered through a pad of silica gel, and eluted with $\mathrm{CH}_{2} \mathrm{Cl}_{2}$. The filtrate was concentrated in vacuo to give a residue, which was purified by a flash column chromatography to afford $\mathbf{4 7 - 4 8}$ and $\mathbf{5 0 - 5 2}$ as white solids.

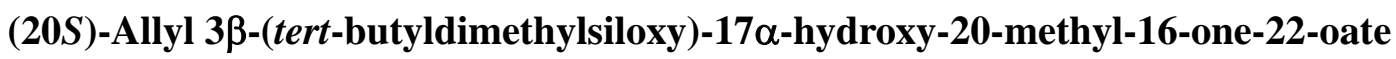
(47)

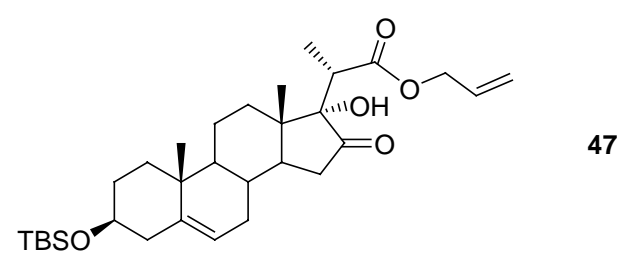

Compound 47 (55\%, two steps from 6). $[\alpha]^{28}{ }_{\mathrm{D}}=-90.2$ (c 1.0, $\mathrm{CHCl}_{3}$ ); ${ }^{1} \mathrm{H}$ NMR (300 
$\mathrm{MHz}, \mathrm{CDCl}_{3}$ ): $\delta$ 6.08-5.92 (m, $\left.1 \mathrm{H}\right)$, 5.42-5.26 (m, $\left.3 \mathrm{H}\right), 5.12$ (br, $\left.1 \mathrm{H}\right), 4.72-4.65$ (m, 2 H), 3.54-3.40 (m, 1 H), 2.59 (q, J = 7.2 Hz, 1 H), 1.02 (s, 3 H), 0.77 (s, 3 H),0.06 (s, 6 H); ${ }^{13} \mathrm{C}$ NMR (75 MHz, $\left.\mathrm{CDCl}_{3}\right): \delta 218.3,180.3,143.5,133.7,122.7,120.7,84.5,74.3,67.6$, 51.2, 47.0, 46.9, 44.6, 40.4, 38.9, 38.5, 37.7, 33.8, 33.7, 32.9, 32.2, 31.6, 27.8, 22.0, 21.3, 20.1, 15.7, 14.4, -2.6.

(20S)-Heptyl 3ß-(tert-butyldimethylsiloxy)-17 $\alpha$-hydroxy-20-methyl-16-one-22-oate (48)

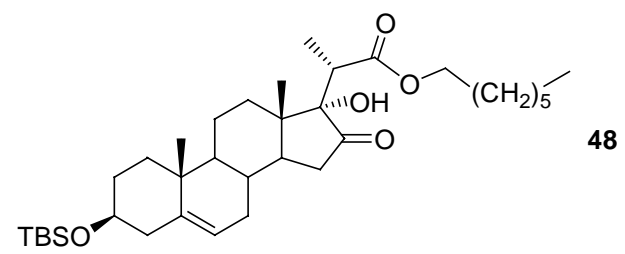

Compound 48 (60\%, two steps from 6): $[\alpha]^{15}=-111.5$ (c 1.1, $\left.\mathrm{CHCl}_{3}\right) ;{ }^{1} \mathrm{H}$ NMR (300 MHz, $\mathrm{CDCl}_{3}$ ): $\delta 5.30$ (m, $\left.1 \mathrm{H}\right), 5.25$ (br, $\left.1 \mathrm{H}\right)$, 4.20-4.10 (m, $\left.2 \mathrm{H}\right), 3.51-3.44(\mathrm{~m}, 1 \mathrm{H})$, 2.55 (q, $J=7.2 \mathrm{~Hz}, 1 \mathrm{H}), 1.00$ (s, $3 \mathrm{H}), 0.91$ (s, $3 \mathrm{H}), 0.76$ (s, $3 \mathrm{H}), 0.05$ (s, $6 \mathrm{H}) ;{ }^{13} \mathrm{C}$ NMR (75 MHz, $\left.\mathrm{CDCl}_{3}\right): \delta$ 216.1, 178.6, 141.4, 120.3, 82.4, 72.2, 65.0, 49.2, 44.9, 44.8, 42.5, 38.4, 36.8, 36.4, 35.6, 31.8, 31.7, 31.5, 30.9, 30.1, 28.7, 28.2, 25.7, 25.6, 22.3, 19.9, 19.2, 18.0, 13.8, 13.6, 12.4, -4.7.

(20S)-Octadecyl 3ß-(tert-butyldimethylsiloxy)-17 $\alpha$-hydroxy-20-methyl-16-one-22oate (50)

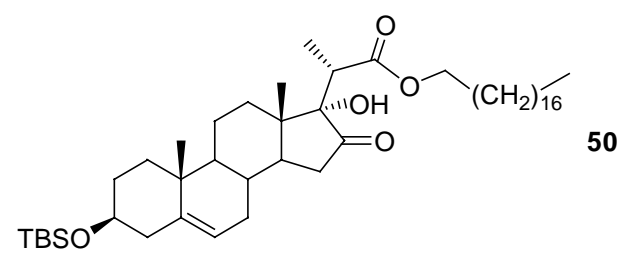

Compound 50 (32\%, two steps from 6): $[\alpha]^{23}{ }_{\mathrm{D}}=-55.1$ (c 0.5, $\left.\mathrm{CHCl}_{3}\right) ;{ }^{1} \mathrm{H}$ NMR (300 MHz, $\mathrm{CDCl}_{3}$ ): $\delta 5.31$ (m, $\left.1 \mathrm{H}\right), 5.26$ (br, $\left.1 \mathrm{H}\right), 4.21-4.12$ (m, $\left.2 \mathrm{H}\right), 3.50$ (m, $\left.1 \mathrm{H}\right), 2.54$ (q, $J=7.2 \mathrm{~Hz}, 1 \mathrm{H}$ ), 1.03 (s, $3 \mathrm{H}), 0.89$ (s, $9 \mathrm{H}), 0.79$ (s, $3 \mathrm{H}), 0.06$ (s, $6 \mathrm{H}) ;{ }^{13} \mathrm{C} \mathrm{NMR}(75$ $\left.\mathrm{MHz}, \mathrm{CDCl}_{3}\right): \delta 216.4,178.9,141.6,120.4,82.5,72.4,65.2,45.1,44.9,42.7,38.5,37.0$, 36.6, 35.8, 31.9, 31.8, 31.0, 30.3, 29.6, 29.6, 29.5, 29.4, 29.3, 29.2, 28.3, 25.9, 25.8, 22.6, 20.1, 19.4, 18.2, 14.1, 13.7, 12.6, -4.5; ESIMS m/z: $765.95\left(\mathrm{M}+\mathrm{Na}^{+}\right)$; HRMS (ESI) $\mathrm{m} / \mathrm{z}$ : 
$765.5810\left(\mathrm{M}+\mathrm{Na}^{+}\right)$; Calcd for $\mathrm{C}_{46} \mathrm{H}_{82} \mathrm{O}_{5} \mathrm{SiNa}$ : 765.5823.

Dodecyl 3 $\beta$-(tert-butyldimethylsiloxy)-17 $\alpha$-hydroxy-16-one-22-oate (51)

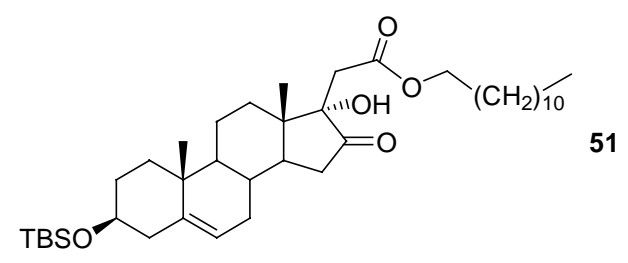

Compound 51 (40\%, for two steps): $[\alpha]^{26}=-95.2$ (c 1.0, $\mathrm{CHCl}_{3}$ ); ${ }^{1} \mathrm{H}$ NMR (300 MHz, $\left.\mathrm{CDCl}_{3}\right): \delta 5.32(\mathrm{~m}, 1 \mathrm{H}), 5.23(\mathrm{br}, 1 \mathrm{H}), 4.22-4.10(\mathrm{~m}, 2 \mathrm{H}), 3.58-3.42(\mathrm{~m}, 1 \mathrm{H}), 2.47$ and 2.34 (AB, $2 \mathrm{H}), 1.02$ (s, $3 \mathrm{H}), 0.71$ (s, $3 \mathrm{H}), 0.06$ (s, $6 \mathrm{H}) ;{ }^{13} \mathrm{C} \mathrm{NMR}\left(75 \mathrm{MHz}, \mathrm{CDCl}_{3}\right): \delta$ 214.8, 174.3, 141.6, 120.4, 80.9, 72.4, 65.4, 49.7, 44.6, 44.5, 42.7, 37.0, 36.7, 35.9, 32.6, 32.0, 31.9, 31.1, 29.6, 29.4, 29.3, 29.1, 28.9, 28.4, 25.9, 22.6, 19.9, 19.4, 18.2, 14.8, 14.0, -4.5 .

(20S)-Isobutyl 3 $\beta$-(tert-butyldimethylsiloxy)-17 $\alpha$-hydroxy-20-methyl-16-one-22thioate (52)

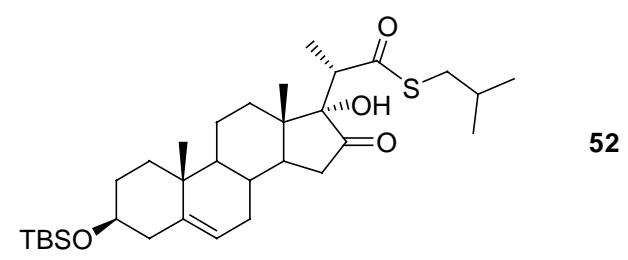

Compound 52 (92\%): $[\alpha]^{18}{ }_{\mathrm{D}}=-100.3\left(\right.$ c 1.5, $\left.\mathrm{CHCl}_{3}\right) ;{ }^{1} \mathrm{H}$ NMR (300 MHz, $\left.\mathrm{CDCl}_{3}\right): \delta$ 5.32 (m, $1 \mathrm{H}), 4.97$ (br, $1 \mathrm{H}), 3.51$ (m, $1 \mathrm{H}), 2.92-2.74$ (m, $3 \mathrm{H}), 1.31$ (q, $J=7.1 \mathrm{~Hz}, 1 \mathrm{H}$ ), 1.01 (s, $3 \mathrm{H}), 0.90$ (s, $9 \mathrm{H}), 0.79$ (s, $3 \mathrm{H}), 0.07$ (s, $6 \mathrm{H}) ;{ }^{13} \mathrm{C} \mathrm{NMR}\left(75 \mathrm{MHz}, \mathrm{CDCl}_{3}\right.$ ): $\delta$ 215.9, 207.6, 141.5, 120.4, 85.2, 72.4, 49.3, 46.8, 45.4, 45.0, 42.7, 37.1, 37.0, 36.6, 35.7, 31.9, 31.8, 31.1, 30.2, 28.6, 25.9, 21.7, 20.1, 19.4, 18.2, 13.9, 13.8, -4.6; ESIMS m/z: $585.45\left(\mathrm{M}+\mathrm{Na}^{+}\right)$; HRMS (ESI) $\mathrm{m} / \mathrm{z}: 585.3405\left(\mathrm{M}+\mathrm{Na}^{+}\right)$; Calcd for $\mathrm{C}_{32} \mathrm{H}_{54} \mathrm{O}_{4} \mathrm{SSiNa}$ : 585.3404.

\section{General procedure for preparation of the $16 \alpha, 17 \beta$-trans-diols $53-55$ and $57-59$}

A suspension of the 16-ketone compound, $\mathrm{CeCl}_{3} \cdot 7 \mathrm{H}_{2} \mathrm{O}(1.4 \mathrm{eq})$, and $\mathrm{NaBH}_{4}(5 \sim 7 \mathrm{eq})$ in dry THF was stirred at $0{ }^{\circ} \mathrm{C}$ for $1 \mathrm{~h}$ and then cooled to $-40 \sim-78^{\circ} \mathrm{C}$ and quenched with 
methanol. After stirring for about $0.5 \mathrm{~h}$, water was added to quenched the reaction completely. The resulting mixture was diluted with $\mathrm{CH}_{2} \mathrm{Cl}_{2}$. The organic layer was washed with $5 \% \mathrm{HCl}$ and brine, respectively, and was then dried over $\mathrm{Na}_{2} \mathrm{SO}_{4}$, and filtered. The filtrate was concentrated in vacuo to give a residue, which was purified by a flash column chromatography to afford 53-55 and 57-59 as white solids.

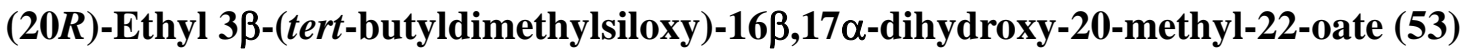

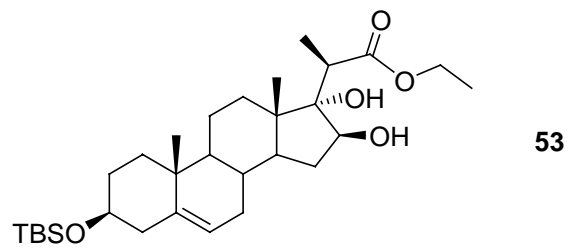

Compound 53 (80\%): $[\alpha]^{18}{ }_{\mathrm{D}}=-31.7$ (c 1.4, $\left.\mathrm{CHCl}_{3}\right)$; ${ }^{1} \mathrm{H}$ NMR (300 MHz, $\left.\mathrm{CDCl}_{3}\right): \delta 5.28$ (m, 1 H), 4.16 (q, J = 7.2 Hz, 2 H), 3.97 (m, 1 H), 3.57 (br, 1 H), 3.55-3.42 (m, 1 H), 2.97 (q, $J=7.1 \mathrm{~Hz}, 1 \mathrm{H}$ ), 1.30 (t, $J=7.2 \mathrm{~Hz}, 3 \mathrm{H}), 1.27$ (d, $J=7.1 \mathrm{~Hz}, 3 \mathrm{H}), 0.98$ (s, $3 \mathrm{H}), 0.90$ (s, $3 \mathrm{H}), 0.86$ (s, $9 \mathrm{H}$ ), 0.04 (s, $6 \mathrm{H}) ;{ }^{13} \mathrm{C} \mathrm{NMR}$ (75 MHz, $\mathrm{CDCl}_{3}$ ): $\delta$ 178.2, 141.8, 121.3, 85.2, 79.2, 72.9, 61.0, 50.0, 48.4, 46.9, 43.1, 39.2, 37.6, 36.9, 36.7, 32.4, 32.1, 32.0, 26.3, 20.7, 19.8, 18.6, 14.5, 14.4, 14.0, -4.3; ESIMS m/z: $519.30\left(\mathrm{M}^{+}-\mathrm{H}\right)$; HRMS (ESI) m/z: $543.3492\left(\mathrm{M}+\mathrm{Na}^{+}\right)$; Calcd for $\mathrm{C}_{30} \mathrm{H}_{52} \mathrm{O}_{5} \mathrm{SiNa}^{+}$: 543.3476 .

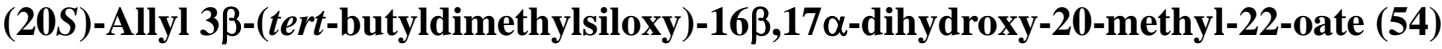

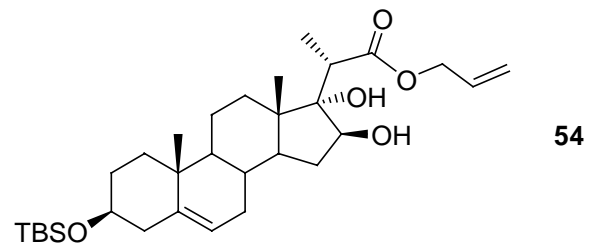

Compound 54 (70\%): ${ }^{1} \mathrm{H}$ NMR (300 MHz, $\left.\mathrm{CDCl}_{3}\right)$ : $\delta$ 5.89-5.80 (m, $\left.1 \mathrm{H}\right)$, 5.33-5.18 (m, 3 H), 4.53 (d, $J=1.8 \mathrm{~Hz}, 2 \mathrm{H}), 3.92$ (m, $1 \mathrm{H}), 3.45-3.32$ (m, $1 \mathrm{H}), 3.32$ (br, $1 \mathrm{H}), 3.05$ (q, $J$ = $7.2 \mathrm{~Hz}, 1 \mathrm{H}), 0.97$ (s, $3 \mathrm{H}), 0.06(\mathrm{~s}, 6 \mathrm{H})$. 


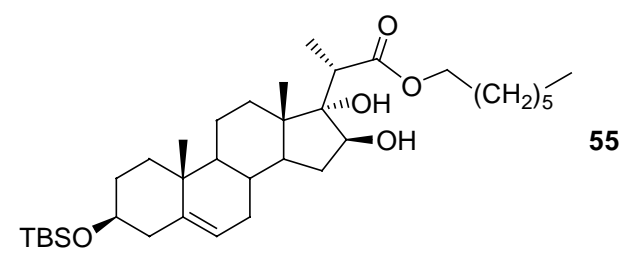

Compound 55 (81\%): $[\alpha]^{18}{ }_{\mathrm{D}}=-36.3\left(\right.$ ( $\left.1.1, \mathrm{CHCl}_{3}\right) ;{ }^{1} \mathrm{H}$ NMR (300 MHz, $\left.\mathrm{CDCl}_{3}\right): \delta 5.24$ (m, 1 H), 4.03 (m, 2 H), 3.91-3.84 (m, 1 H), 3.48-3.36 (m, 1 H), 3.32 (br, 1 H), 3.10 (q, $J$ $=7.4 \mathrm{~Hz}, 1 \mathrm{H}), 0.91$ (s, $3 \mathrm{H}), 0.83$ (s, $3 \mathrm{H}), 0.81$ (s, $3 \mathrm{H}), 0.06(\mathrm{~s}, 6 \mathrm{H}) ;{ }^{13} \mathrm{C}$ NMR $(75$ $\left.\mathrm{MHz}, \mathrm{CDCl}_{3}\right): \delta 178.5,141.5,120.8,84.8,81.7,72.5,64.9,49.6,48.3,46.2,42.7,41.0$, 37.2, 36.5, 35.7, 32.4, 32.0, 31.8, 31.6, 29.6, 28.8, 28.5, 25.9, 25.8, 22.5, 20.4, 19.3, 18.2, $14.0,13.4,12.3,-4.5$.

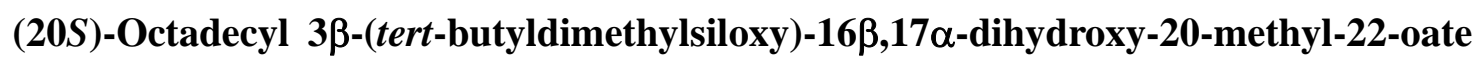
(57)

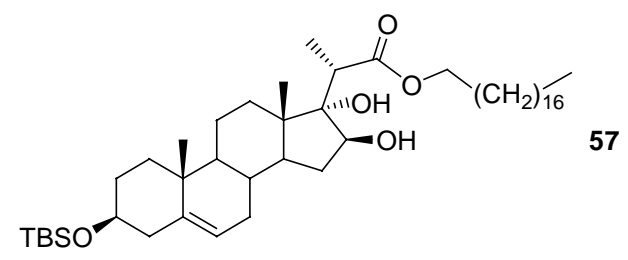

Compound 57 (84\%): $[\alpha]^{20}{ }_{\mathrm{D}}=-20.7$ (c 0.56, $\left.\mathrm{CHCl}_{3}\right) ;{ }^{1} \mathrm{H}$ NMR (300 MHz, $\left.\mathrm{CDCl}_{3}\right): \delta$ 5.30 (m, 1 H), 4.13-4.07 (m, 2 H), 4.97 (m, 1 H), 3.55-3.43 (m, 1 H), 3.40 (br, 1 H), 3.07 (q, $J=7.2 \mathrm{~Hz}, 1 \mathrm{H}), 1.01$ (s, $3 \mathrm{H}), 0.06$ (s, $6 \mathrm{H})$; ESIMS m/z: $767.80\left(\mathrm{M}+\mathrm{Na}^{+}\right.$).

\section{Dodecyl 3 $\beta$-(tert-butyldimethylsiloxy)-16 $\beta, 17 \alpha$-dihydroxy-22-oate (58)}

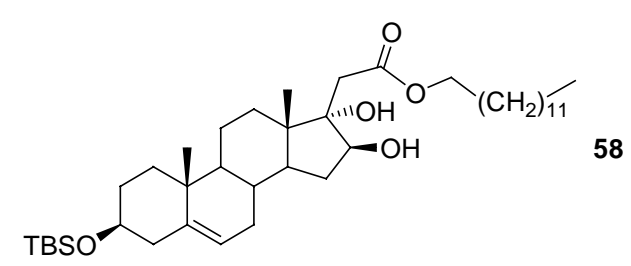

Compound 58 (84\%): $[\alpha]^{26}{ }_{\mathrm{D}}=-31.9\left(\right.$ ( $\left.0.6, \mathrm{CHCl}_{3}\right) ;{ }^{1} \mathrm{H}$ NMR (300 MHz, $\left.\mathrm{CDCl}_{3}\right): \delta 5.31$ (m, $1 \mathrm{H}), 4.12$ (t, $J=6.6 \mathrm{~Hz}, 2 \mathrm{H}), 4.06(\mathrm{~m}, 1 \mathrm{H}), 3.51-3.43(\mathrm{~m}, 1 \mathrm{H}), 3.13$ (d, $J=3.0 \mathrm{~Hz}$, $1 \mathrm{H}), 2.83$ and 2.45 (AB, $2 \mathrm{H}), 2.41$ (br, $1 \mathrm{H}), 1.00$ (s, $3 \mathrm{H}), 0.81$ (s, $3 \mathrm{H}), 0.06$ (s, $6 \mathrm{H})$; ${ }^{13} \mathrm{C} \mathrm{NMR}\left(75 \mathrm{MHz}, \mathrm{CDCl}_{3}\right): \delta 174.5,141.4,120.8,84.0,80.6,72.5,65.3,49.9,47.6,45.8$, 42.7, 37.3, 36.7, 36.6, 34.8, 32.0, 31.9, 31.8, 31.7, 30.6, 29.6, 29.6, 29.5, 29.4, 29.3, 29.1, 


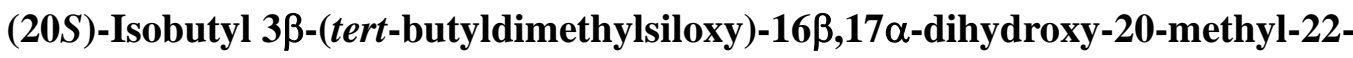
thioate (59)

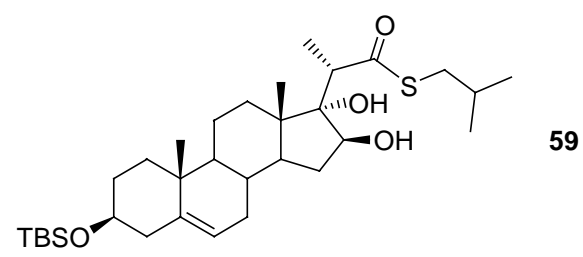

Compound 59 (43\%): $[\alpha]^{18}{ }_{\mathrm{D}}=-22.7\left(\mathrm{c} \mathrm{0.5}, \mathrm{CHCl}_{3}\right)$; ${ }^{1} \mathrm{H}$ NMR (300 MHz, $\left.\mathrm{CDCl}_{3}\right): \delta 5.31$ (m, 1 H), 3.97-3.91 (m, 1 H), 3.52 (br, 1 H), 3.52-3.45 (m, 1 H), 3.29 (q, J = 7.2 Hz, 1 H), 2.88-2,74 (m, 2 H), 1.36 (d, J = 7.2 Hz, 3 H), 1.02 (s, 3 H), 0.99 (s, 3 H), 0.97 (s, 3 H), 0.91 (s, $3 \mathrm{H}$ ), 0.90 (s, $9 \mathrm{H}$ ), 0.06 (s, $6 \mathrm{H}$ ); ${ }^{13} \mathrm{C} \mathrm{NMR} \mathrm{(75} \mathrm{MHz,} \mathrm{CDCl}_{3}$ ): $\delta$ 207.9, 141.5, 120.9, 86.1, 81.6, 76.6, 72.5, 49.6, 49.2, 48.3, 46.5, 42.8, 37.3, 37.0, 36.5, 35.7, 32.4, 32.0. 31.9, 28.8, 25.9, 21.7, 20.4, 19.4, 18.3, 13.8, 13.5, -4.6; ESIMS m/z: $587.55\left(\mathrm{M}+\mathrm{Na}^{+}\right)$; HRMS (ESI) m/z: $587.3538\left(\mathrm{M}+\mathrm{Na}^{+}\right)$; Calcd for $\mathrm{C}_{32} \mathrm{H}_{56} \mathrm{O}_{4} \mathrm{SSiNa}$ : 587.3561.

\section{General procedure for preparation of compounds 61-63 and 65-67 by glycosylation}

A solution of the disaccharide donor 33 (1.2 eq), aglycone (1 eq), and $4 \AA$ MS in dry $\mathrm{CH}_{2} \mathrm{Cl}_{2}$ was stirred at $\mathrm{rt}$ for $15 \mathrm{~min}$, and then cooled to $-20^{\circ} \mathrm{C}$. A solution of TMSOTf $(0.1$ eq) in $\mathrm{CH}_{2} \mathrm{Cl}_{2}$ was added slowly to the mixture. After stirring for $1 \mathrm{~h}$, the reaction was quenched with $\mathrm{Et}_{3} \mathrm{~N}$ and filtered. The filtrates were concentrated in vacuo to give a residue, which was purified by a flash column chromatography to afford glycosides 61-63 and 65-67 as white foams.

\section{Protected (20R)-ethyl-23-oxa-OSW-1 (61)}

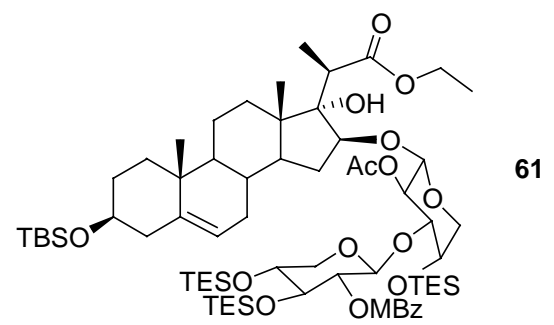

This compound was contaminated by an unidentified by-product derived from the 
disaccharide donor, and was directly subjected to the next transformation.

Protected (20S)-allyl-23-oxa-OSW-1 (62)

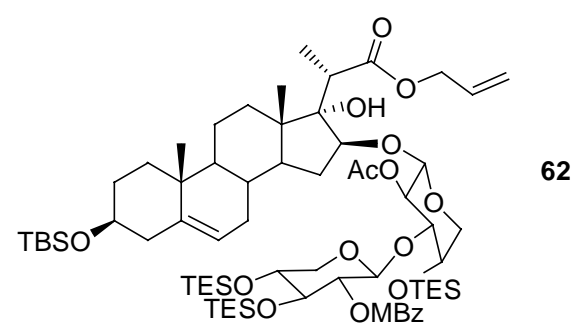

Compound 62 (57\%): ${ }^{1} \mathrm{H}$ NMR (300 MHz, $\mathrm{CDCl}_{3}$ ): $\delta 8.05$ and 6.91 (AB, $\left.4 \mathrm{H}\right), 5.69$ (m, 1 H), 5.32 (m, 1 H), 5.13-4.96 (m, 4 H), 4.34-4.28 (m, 3 H), 3.88 (s, 3 H), 2.82 (q, J = 7.2 Hz, 1 H), 2.00 (s, 3 H), 0.06 (s, $6 \mathrm{H})$; ESIMS m/z: $1337.7\left(\mathrm{M}+\mathrm{Na}^{+}\right)$.

\section{Protected (20S)-heptyl-23-oxa-OSW-1 (63)}

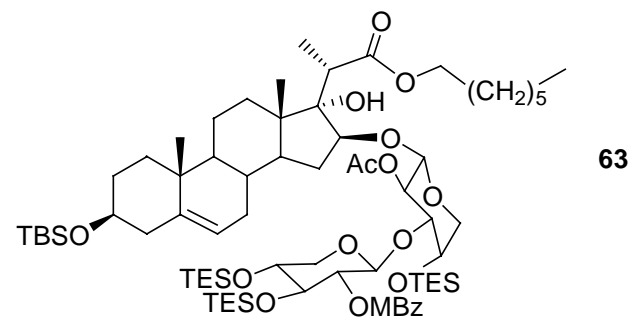

Compound 63 (64\%): $[\alpha]^{18}=-41.0$ (c 1.0, $\left.\mathrm{CHCl}_{3}\right) ;{ }^{1} \mathrm{H}$ NMR (300 MHz, $\left.\mathrm{CDCl}_{3}\right): \delta 8.07$ and 6.91 (AB, $4 \mathrm{H}), 5.31$ (m, $1 \mathrm{H}), 4.97$ (br, $1 \mathrm{H}), 4.87$ (m, $1 \mathrm{H}), 4.80$ (m, $1 \mathrm{H}), 3.85$ (s, 3 H), 2.80 (q, $J=7.1 \mathrm{~Hz}, 1 \mathrm{H}), 2.00$ (s, $3 \mathrm{H}), 0.05$ (s, $6 \mathrm{H}) ;{ }^{13} \mathrm{C} \mathrm{NMR}\left(75 \mathrm{MHz}, \mathrm{CDCl}_{3}\right.$ ): $\delta$ 180.4, 170.0, 165.6, 164.2, 142.3, 132.7, 123.5, 121.8, 113.9, 101.2, 85.2, 72.5, 71.3, 69.4, 68.4, 64.6, 55.3, 49.5, 48.2, 45.7, 42.8, 40.7, 37.2, 36.5, 34.8, 32.0, 31.5, 29.6, 28.8, 28.3, 25.9, 25.4, 22.5, 20.7, 20.5, 19.1, 18.2, 13.9, 12.9, 6.9, 6.7, 4.8, 4.8, 4.7, -4.5; ESIMS m/z: 1396.25 $\left(\mathrm{M}+\mathrm{Na}^{+}\right)$; HRMS (ESI) $\mathrm{m} / \mathrm{z}: 1395.8152\left(\mathrm{M}+\mathrm{Na}^{+}\right)$; Calcd for $\mathrm{C}_{73} \mathrm{H}_{128} \mathrm{O}_{16} \mathrm{Si}_{4} \mathrm{Na}$ : 1395.8171.

Protected (20S)-octadecyl-23-oxa-OSW-1 (65) 


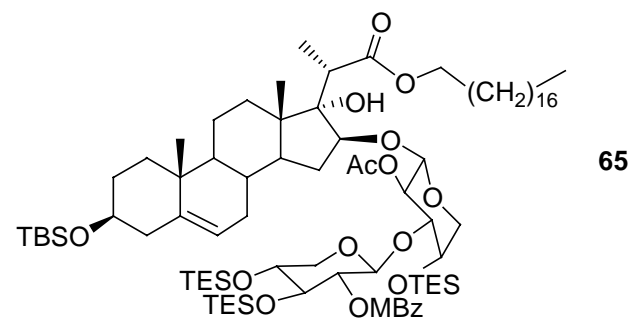

Compound 65 (50\%): $[\alpha]^{20}{ }_{\mathrm{D}}=-32.8\left(\mathrm{c}\right.$ 0.96, $\left.\mathrm{CHCl}_{3}\right) ;{ }^{1} \mathrm{H}$ NMR (300 MHz, $\left.\mathrm{CDCl}_{3}\right): \delta$ 8.07 and 6.89 (AB, 4 H), 5.31 (m, 1 H), 4.97 (br, 1 H), 4.87 (m, 1 H), 4.80 (m, 1 H), 3.87 (s, $3 \mathrm{H}), 2.80$ (q, $J=7.2 \mathrm{~Hz}, 1 \mathrm{H}), 2.00$ (s, $3 \mathrm{H}), 0.06(\mathrm{~s}, 6 \mathrm{H}) ;{ }^{13} \mathrm{C}$ NMR $(75 \mathrm{MHz}$, $\left.\mathrm{CDCl}_{3}\right): \delta 179.4,168.9,164.6,163.2,141.4,131.9,122.8,121.1,113.1,100.6,84.7,72.6$, 71.4, 69.4, 68.5, 64.7, 55.3, 49.5, 48.3, 45.7, 42.8, 40.7, 37.2, 36.5, 34.8, 32.0, 31.9, 29.7, 29.4, 29.3, 29.3, 28.3, 25.9, 25.5, 22.6, 20.8, 20.5, 19.1, 18.2, 14.0, 13.9, 12.9, 6.9, 6.7, 4.9, 4.8, 4.7, -4.5; ESIMS m/z: $1551.05\left(\mathrm{M}+\mathrm{Na}^{+}\right)$.

Protected dodecyl-21-nor-23-oxa-OSW-1 (66)

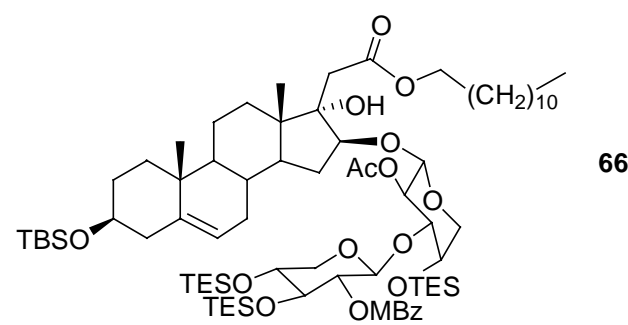

Compound 66 (61\%): $[\alpha]^{28}=-14.4\left(c\right.$ 1.0, $\left.\mathrm{CHCl}_{3}\right) ;{ }^{1} \mathrm{H}$ NMR (300 MHz, $\left.\mathrm{CDCl}_{3}\right): \delta 8.00$ and $6.92(\mathrm{AB}, 4 \mathrm{H}), 5.29(\mathrm{~m}, 1 \mathrm{H}), 4.96-4.87(\mathrm{~m}, 2 \mathrm{H}), 4.74(\mathrm{~d}, J=5.1 \mathrm{~Hz}, 1 \mathrm{H}), 4.26$ (d, $J=5.1 \mathrm{~Hz}, 1 \mathrm{H}), 3.95$ (s, $3 \mathrm{H}), 3.35-3.19$ (m, 2 H), 2.59 and 2.38 (AB, 2 H), 1.96 (s, $3 \mathrm{H}$ ), 0.06 (s, $6 \mathrm{H})$; ESIMS m/z: $1451.9\left(\mathrm{M}+\mathrm{Na}^{+}\right)$; HRMS (ESI) m/z: $1451.8813\left(\mathrm{M}+\mathrm{Na}^{+}\right)$; Calcd for $\mathrm{C}_{77} \mathrm{H}_{136} \mathrm{O}_{16} \mathrm{Si}_{4} \mathrm{Na}$ : 1451.8797 .

\section{Protected (20S)-23-thio-OSW-1 (67)}

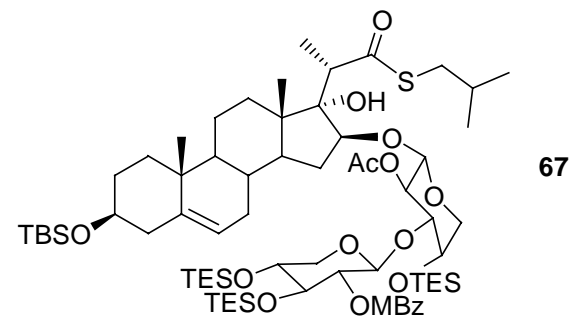


Compound 67: $[\alpha]^{16}{ }_{\mathrm{D}}=-35.4\left(c\right.$ 1.3, $\left.\mathrm{CHCl}_{3}\right){ }^{1} \mathrm{H}$ NMR $\left(500 \mathrm{MHz}, \mathrm{CDCl}_{3}\right): \delta 8.11$ and 6.89 (AB, 4 H), 5.32 - 5.31 (m, 1 H), 5.19 (m, 1 H), 5.17 - 5.16 (m, 1 H), 4.92(m, 1 H), $4.44-4.40$ (m, $1 \mathrm{H}), 4.28$ (s, $1 \mathrm{H}$ ), 3.87 (s, $3 \mathrm{H}$ ), 3.12 (q, $J=7.8 \mathrm{~Hz}, 1 \mathrm{H}$ ), 2.90 (dd, $J=$ 6.1 Hz, 13.4 Hz, 1 H), 2.35 (dd, $J=7.2 \mathrm{~Hz}, 13.4 \mathrm{~Hz}, 1 \mathrm{H}$ ), 2.01 (s, $3 \mathrm{H}), 0.91$ (s, $3 \mathrm{H}$ ), 0.90 (s, 9 H), 0.89 (s, $3 \mathrm{H}), 0.06$ (s, $6 \mathrm{H})$; MALDI-MS m/z: $1370.3\left(\mathrm{M}+\mathrm{Na}^{+}\right)$; HRMS (MALDI) m/z: $1369.7457\left(\mathrm{M}+\mathrm{Na}^{+}\right)$; Calcd for $\mathrm{C}_{70} \mathrm{H}_{122} \mathrm{O}_{15} \mathrm{SSi}_{4} \mathrm{Na}$ : 1369.7474 .

\section{General procedure for preparation of compounds 69,71 , and 73-75 by deprotection}

A solution of the protected OSW-1 analogue (1 equiv) and $\mathrm{Pd}\left(\mathrm{MeCN}_{2} \mathrm{Cl}_{2}\right.$ (cat.) in acetone and water (v:v, 20:1) was stirred at rt until TLC indicated the reaction has finished. The solution was then directly concentrated in vacuo to give a residue, which was purified by a flash column chromatography $\left(\mathrm{CH}_{2} \mathrm{Cl}_{2}-\mathrm{MeOH}\right.$ system) to give $\mathbf{6 9}, \mathbf{7 1}$, and 73-75 as white solids.

(20R)-Ethyl-23-oxa-OSW-1 (69)

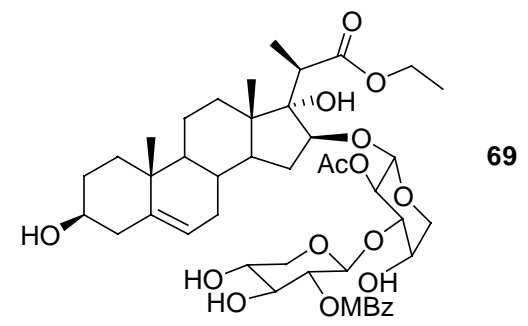

Compound 69 (69\%): $[\alpha]^{20}{ }_{\mathrm{D}}=-51.8\left(c\right.$ 0.23, $\left.\mathrm{CHCl}_{3}\right) ;{ }^{1} \mathrm{H}$ NMR $\left(300 \mathrm{MHz}, \mathrm{CDCl}_{3}\right): \delta$ 7.97 (d, $J=9.0 \mathrm{~Hz}, 2 \mathrm{H}$ ), 6.91 (d, $J=9.0 \mathrm{~Hz}, 2 \mathrm{H}$ ), 5.32 (m, $1 \mathrm{H}$ ), 5.12 (dd, $J=6.8,9.4$ Hz, $1 \mathrm{H}$ ), 4.99 (dd, $J=7.2,7.8 \mathrm{~Hz}, 1 \mathrm{H}$ ), 4.70 (d, $J=6.8 \mathrm{~Hz}, 1 \mathrm{H}$ ), 4.27 (d, $J=7.2 \mathrm{~Hz}, 1$ H), 3.86 (s, 3 H), 2.66 (q, $J=6.9 \mathrm{~Hz}, 1 \mathrm{H}), 1.66$ (s, $3 \mathrm{H}), 1.06$ (d, $J=6.9 \mathrm{~Hz}$ ), 0.97 (s, 3 $\mathrm{H}), 0.80(\mathrm{~s}, 3 \mathrm{H}) ;{ }^{13} \mathrm{C}$ NMR $\left(125 \mathrm{MHz}, \mathrm{CDCl}_{3}\right): \delta 177.7,169.3,165.9,164.0,140.5$, 132.1, 121.6, 121.5, 113.8, 102.8, 101.7, 87.7, 85.3, 80.5, 76.8, 74.4, 73.8, 71.8, 70.5, 69.8, 67.5, 64.6, 60.6, 55.5, 49.7, 48.3, 46.4, 42.3, 38.7, 37.2, 36.4, 35.0, 31.7, 29.7, 20.6, 20.4, 19.4, 14.1, 14.0, 13.9, 13.5; ESIMS m/z: $869.8\left(\mathrm{M}+\mathrm{Na}^{+}\right)$; HRMS (ESI) m/z: 869.3920 $\left(\mathrm{M}+\mathrm{Na}^{+}\right)$; Calcd for $\mathrm{C}_{44} \mathrm{H}_{62} \mathrm{O}_{16} \mathrm{Na}$ : 869.3930.

(20S)-Heptyl-23-oxa-OSW-1 (71) 


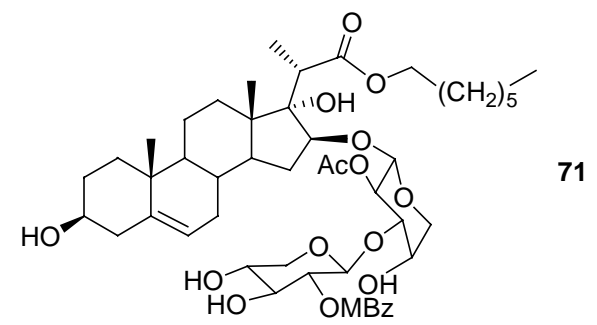

Compound 71 (95\%): $[\alpha]^{19}{ }_{\mathrm{D}}=-49.3\left(\mathrm{c} \mathrm{0.9}, \mathrm{CHCl}_{3}\right)$; ${ }^{1} \mathrm{H}$ NMR (300 MHz, $\left.\mathrm{CDCl}_{3}\right): \delta 8.02$ and $6.93(\mathrm{AB}, 4 \mathrm{H}), 5.32(\mathrm{~m}, 1 \mathrm{H}), 4.93$ (t, $J=5.5 \mathrm{~Hz}, 1 \mathrm{H}), 4.85$ (d, $J=5.4 \mathrm{~Hz}, 1 \mathrm{H})$, 4.79 (m, 1 H), 4.24 (m, 1 H), 3.85 (s, 3 H), 2.75 (br, 1 H), 2.62 (q, J = 7.1 Hz, 1 H), 1.98 (s, $3 \mathrm{H}), 0.80$ (s, $3 \mathrm{H}) ;{ }^{13} \mathrm{C}$ NMR $\left(75 \mathrm{MHz} \mathrm{CDCl}_{3}\right): \delta 178.9,169.5,165.6,163.8,140.5$, 132.0, 121.4, 113.7, 100.1, 100.0, 89.5, 84.6, 73.2, 72.7, 71.7, 69.8, 69.2, 64.8, 64.4, 63.6, 61.5, 55.4, 49.4, 48.2, 45.7, 42.2, 40.5, 37.1, 36.3, 34.7, 32.0, 31.6, 29.6, 28.8, 28.3, 25.5, 22.5, 20.6, 19.4, 14.0, 13.2, 12.7; ESIMS m/z: $939.8\left(\mathrm{M}+\mathrm{Na}^{+}\right)$; HRMS (ESI) $\mathrm{m} / z$ : 939.4736 $\left(\mathrm{M}+\mathrm{Na}^{+}\right)$; Calcd for $\mathrm{C}_{49} \mathrm{H}_{72} \mathrm{O}_{16} \mathrm{Na}$ : 939.4712.

(20S)-Octadecyl-23-oxa-OSW-1 (73)

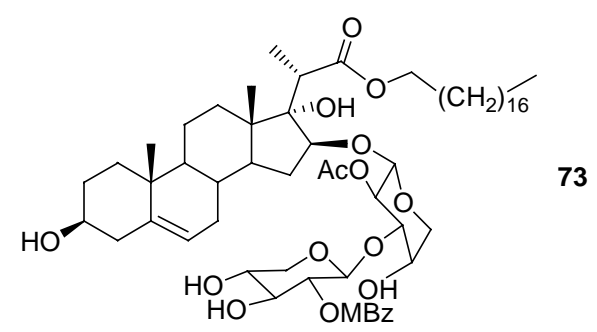

Compound 73 (90\%): $[\alpha]^{20}{ }_{\mathrm{D}}=-26.6\left(\right.$ c $\left.0.9,_{\mathrm{CHCl}_{3}}\right) ;{ }^{1} \mathrm{H}$ NMR (300 MHz, $\left.\mathrm{CDCl}_{3}\right): \delta 8.06$ and 6.96 (AB, $4 \mathrm{H}), 5.33$ (m, $1 \mathrm{H}), 4.95$ (t, $J=5.4 \mathrm{~Hz}, 1 \mathrm{H}), 4.87$ (d, $J=5.4 \mathrm{~Hz}, 1 \mathrm{H}$ ), 4.80 (m, $1 \mathrm{H}$ ), 4.26 (d, $J=2.1 \mathrm{~Hz}, 1 \mathrm{H}$ ), 3.87 (s, $3 \mathrm{H}$ ), 2.68-2.60 (m, $2 \mathrm{H}$ ), 2.00 (s, $3 \mathrm{H}$ ), 0.81 (s, $3 \mathrm{H}) ;{ }^{13} \mathrm{C}$ NMR $\left(75 \mathrm{MHz}, \mathrm{CDCl}_{3}\right): \delta 178.9,169.5,165.7,163.8,140.5,132.1$, 121.5, 121.3, 113.8, 113.6, 100.1, 99.9, 89.6, 84.6, 73.3, 72.8, 71.7, 69.8, 69.2, 64.6, 64.4, 63.6, 61.2, 55.4, 49.4, 48.2, 45.7, 42.2, 40.5, 37.1, 36.4, 34.7, 32.7, 32.0, 31.9, 31.7, 31.5, 30.3, 29.6, 29.3, 28.3, 25.8, 25.6, 22.6, 20.7, 20.5, 19.7, 19.4, 14.1, 13.2, 12.7; ESIMS m/z: $1093.9\left(\mathrm{M}+\mathrm{Na}^{+}\right)$; HRMS (ESI) m/z: $1093.6417\left(\mathrm{M}+\mathrm{Na}^{+}\right)$; Calcd for $\mathrm{C}_{60} \mathrm{H}_{94} \mathrm{O}_{16} \mathrm{Na}$ : 1093.6434.

\section{Dodecyl-23-oxa-OSW-1 (74)}




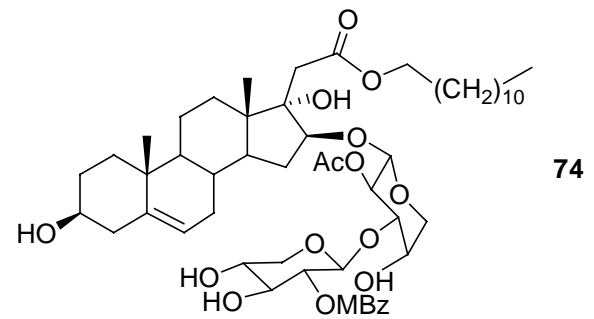

Compound 74 (85\%): $[\alpha]^{28}=-14.4$ (c 1.0, $\left.\mathrm{CHCl}_{3}\right)$; ${ }^{1} \mathrm{H}$ NMR (300 MHz, $\left.\mathrm{CDCl}_{3}\right): \delta 7.94$ and $6.90(\mathrm{AB}, 4 \mathrm{H}), 5.32$ (m, $1 \mathrm{H}), 5.00-4.95(\mathrm{~m}, 2 \mathrm{H}), 4.70(\mathrm{~m}, 1 \mathrm{H}), 4.27$ (d, $J=6.0 \mathrm{~Hz}$, $1 \mathrm{H}), 4.15-4.08$ (m, $1 \mathrm{H}), 3.84$ (s, $3 \mathrm{H}), 2.83$ (br, $1 \mathrm{H}), 1.70$ (s, $3 \mathrm{H}), 0.67$ (s, $3 \mathrm{H}) ;{ }^{13} \mathrm{C}$ NMR (75 MHz, $\mathrm{CDCl}_{3}$ ): $\delta$ 174.5, 169.4, 165.8, 163.9, 140.5, 132.1, 121.5, 121.4, 113.7, 101.6, 101.2, 88.5, 82.9, 79.6, 74.0, 73.3, 71.7, 70.3, 69.5, 66.9, 64.8, 64.4, 63.8, 55.4, 53.4, 49.9, 49.4, 47.7, 45.5, 42.2, 37.2, 36.5, 35.2, 35.0, 31.9, 31.8, 31.6, 30.7, 30.6, 29.7, 29.6, 29.6, 29.5, 29.3, 29.2, 28.4, 25.8, 22.7, 20.3, 19.4, 17.6, 14.1; ESIMS m/z: 995.7 (M $\left.+\mathrm{Na}^{+}\right)$; HRMS (ESI) m/z: $995.5358\left(\mathrm{M}+\mathrm{Na}^{+}\right)$; Calcd for $\mathrm{C}_{53} \mathrm{H}_{80} \mathrm{O}_{16} \mathrm{Na}$ : 995.5338.

\section{3-Thio-OSW-1 (75)}

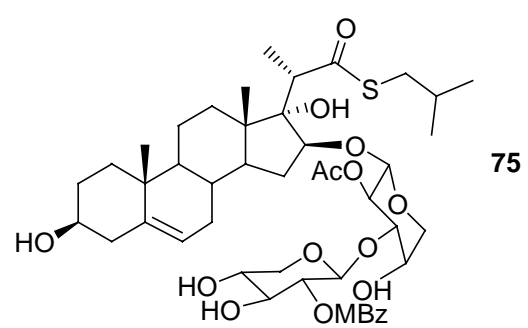

Compound 75 was obtained as a pale yellow solid (44\% for the last two steps): $[\alpha]^{17}{ }_{\mathrm{D}}=$ -32.2 (c 0.25, $\mathrm{CHCl}_{3}$ ); ${ }^{1} \mathrm{H}$ NMR (300 MHz, $\left.\mathrm{CDCl}_{3}\right): \delta 8.11$ and $6.98(\mathrm{AB}, 4 \mathrm{H}), 5.34$ (m, 1 H), 5.09 (m, 1 H), 4.96-4.91 (m, 2 H), 4.23 (br, 1 H), 4.18 (m, 1 H), 3.98 (1 H), 3.87 (s, 3 H), 2.88-2.79 (m, 3 H), 2.70 (br, 1 H), 2.04 (s, 3 H), 1.13 (d , J = 7.3 Hz, 3 H), 1.23 (s, $3 \mathrm{H}), 1.03$ (s, $3 \mathrm{H}), 0.80$ (s, $3 \mathrm{H}) ;{ }^{13} \mathrm{C}$ NMR (125 MHz, $\left.\mathrm{CDCl}_{3}\right): \delta$ 208.2, 169.2, 166.2, 164.1, 140.7, 132.4, 121.5, 121.4, 114.1, 100.6, 99.6, 89.1, 86.3, 76.8, 74.0, 73.2, 71.8, 70.6, 69.6, 63.8, 63.7, 55.5, 49.6, 48.9, 48.2, 46.1, 42.3, 36.6, 36.5, 34.6, 32.1, 31.9, 28.5, 21.8, 21.6, 20.9, 19.5, 14.5, 13.4; ESIMS m/z: $913.7\left(\mathrm{M}+\mathrm{Na}^{+}\right)$; HRMS (ESI) $\mathrm{m} / z$ : 913.4017 $\left(\mathrm{M}+\mathrm{Na}^{+}\right)$; Calcd for $\mathrm{C}_{46} \mathrm{H}_{66} \mathrm{O}_{15} \mathrm{SNa}$ : 913.4015 . 


\section{Amide 77}

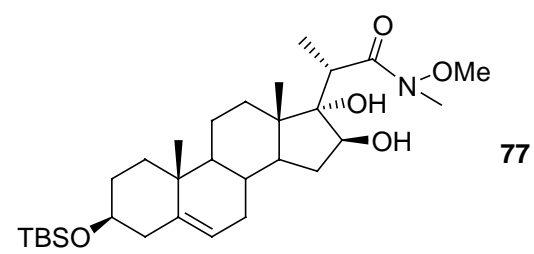

A solution of DIBAL-H (1 M in toluene, $1.96 \mathrm{~mL}, 1.96 \mathrm{mmol})$ was added to a cool $\left(0^{\circ} \mathrm{C} 5^{\circ} \mathrm{C}\right)$ solution of $(\mathrm{MeO}) \mathrm{MeNH} \cdot \mathrm{HCl}(195 \mathrm{mg}, 2 \mathrm{mmol})$ in THF (2 mL) under argon. The mixture was allowed to warm up and stirred at rt for $2 \mathrm{~h}$. The concentration of the prepared DIBAL-H/(MeO)MeNH•HCl complex was about $0.5 \mathrm{~mol} / \mathrm{L}$.

To a solution of 26 (35 mg, $0.074 \mathrm{mmol}$ ) in THF ( $2 \mathrm{~mL}$ ) was added, under Ar at rt, the DIBAL-H/(MeO)MeNH• $\mathrm{HCl}$ complexes $(0.9 \mathrm{~mL}, 0.5 \mathrm{~mol} / \mathrm{L})$. After stirring at rt for $2 \mathrm{~h}$, the reaction was cooled to $0{ }^{\circ} \mathrm{C}$, and then quenched with $\mathrm{H}_{2} \mathrm{O}(0.1 \mathrm{~mL})$ and a $1 \mathrm{~N}$ aqueous solution of $\mathrm{KHSO}_{4}(5 \mathrm{~mL})$. The resulting mixture was extracted with $\mathrm{CH}_{2} \mathrm{Cl}_{2}$. The combined organic layers were washed with brine, dried over $\mathrm{Na}_{2} \mathrm{SO}_{4}$, and concentrated. The residue was purified by a flash chromatography (petroleum ether:EtOAc, 3:1) to afford 77 (36 mg, 91\%) as a white solid. $[\alpha]^{18}{ }_{\mathrm{D}}=-22.3$ (c 1.3, $\mathrm{CHCl}_{3}$ ); ${ }^{1} \mathrm{H}$ NMR (300 MHz, $\mathrm{CDCl}_{3}$ ): $\delta 5.31$ (m, 1 H), 5.02 (br, 1 H), 4.06 (m, 1 H), 3.76 (s, 3 H), 3.52-3.45 (m, 1 H), 3.37 (q, $J=7.1$ Hz, 1 H), 3.18 (s, 3 H), 1.25 (d, $J=7.1$ Hz, 3 H), 1.01 (s, 3 H), 0.90 (s, $3 \mathrm{H}$ ), 0.89 (s, $9 \mathrm{H}), 0.06$ (s, $6 \mathrm{H}) ;{ }^{13} \mathrm{C}$ NMR (75 MHz, $\mathrm{CDCl}_{3}$ ): $\delta$ 180.2, 141.5, 120.9, 85.2, 81.9, 72.5, 61.0, 49.6, 48.1, 46.2, 42.8, 37.3, 36.6, 36.4, 35.7, 32.6, 32.0, 31.9, 29.7, 25.9, 20.5, 19.4, 18.2, 13.7, 12.3, -4.6; ESIMS m/z: $558.4\left(\mathrm{M}+\mathrm{Na}^{+}\right)$; HRMS (ESI) m/z: $558.3584\left(\mathrm{M}+\mathrm{Na}^{+}\right)$; Calcd for $\mathrm{C}_{30} \mathrm{H}_{53} \mathrm{NO}_{5} \mathrm{SiNa}$ : 558.3585 .

\section{Protected 23-Aza-OSW-1 analogue (79)}




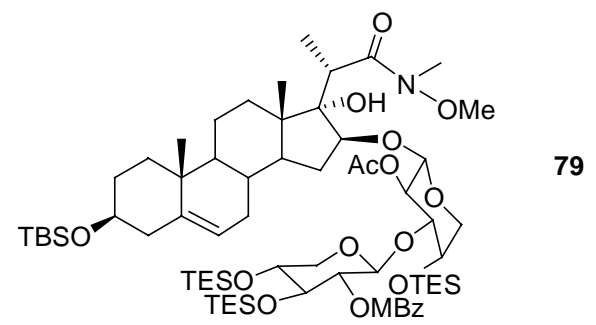

Compound 79 (61\%) was prepared employing a similar procedure as described for the preparation of $\mathbf{6 1}$ by glycosylation. $[\alpha]^{20}{ }_{\mathrm{D}}=-32.3$ (c 1.3, $\left.\mathrm{CHCl}_{3}\right) ;{ }^{1} \mathrm{H}$ NMR (300 MHz, $\left.\mathrm{CDCl}_{3}\right): \delta 8.04$ and $6.90(\mathrm{AB}, 4 \mathrm{H}), 5.32(\mathrm{~m}, 1 \mathrm{H}), 5.18(\mathrm{~m}, 1 \mathrm{H}), 4.90(\mathrm{~m}, 2 \mathrm{H}), 4.83(\mathrm{~m}$, 1 H), 4.37 (d, 1 H), 4.31 (br, 1 H), 3.87 (s, 3 H), 3.49 (s, 3 H), 2.95 (s, 3 H), 1.91 (s, 3 H), 1.20 (d, $J=6.9 \mathrm{~Hz}, 3 \mathrm{H}), 0.06$ (s, $\left.6 \mathrm{H}) ;{ }^{13} \mathrm{C} \mathrm{NMR} \mathrm{(75} \mathrm{MHz,} \mathrm{CDCl}_{3}\right): \delta$ 179.6, 169.0, 164.6, 163.4, 141.4, 132.0, 122.8, 121.0, 113.4, 99.6, 89.9, 85.3, 72.6, 71.8, 69.9, 60.9, 55.4, 49.6, 48.1, 46.1, 42.9, 37.2, 36.5, 35.9, 32.4, 32.1, 31.9, 31.7, 25.9, 20.8, 20.6, 19.3, 18.2, 13.9, 12.6, 6.9, 6.7, 5.0, 4.8, 4.8, -4.6; MALDI-MS m/z: $1340.8\left(\mathrm{M}+\mathrm{Na}^{+}\right)$; HRMS (MALDI) m/z: $1340.7513\left(\mathrm{M}+\mathrm{Na}^{+}\right)$; Calcd for $\mathrm{C}_{68} \mathrm{H}_{119} \mathrm{NaNO}_{16} \mathrm{Si}_{4}: 1340.7498$.

\section{3-Aza-OSW-1 analogue (81)}

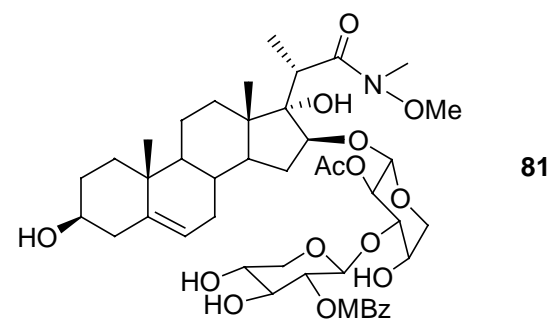

Compound 81 (86\%) was prepared employing a similar procedure as described for the preparation of 69 by deprotection. $[\alpha]^{20}{ }_{\mathrm{D}}=-16.5$ (c 0.9, $\left.\mathrm{CHCl}_{3}\right) ;{ }^{1} \mathrm{H}$ NMR $(400 \mathrm{MHz}$, $\left.\mathrm{CDCl}_{3}\right): \delta 7.98$ and $6.93(\mathrm{AB}, 4 \mathrm{H}), 5.34(\mathrm{~m}, 1 \mathrm{H}), 5,27(1 \mathrm{H}), 5.01(\mathrm{~m}, 1 \mathrm{H}), 4.91(\mathrm{~m}, 1$ H), 4.84 (m, 1 H), 4.34 (d, J = 5.3 Hz, 1 H), 4.20 (m, 1 H), 3.87 (s, 3 H), 3.61 (s, 3 H), 3.26 (q, $J=7.2$ Hz, 1 H), 3.01 (s, 3 H), 1.77 (s, 3 H), 1.19 (d, $J=7.1$ Hz, 3 H), 1.01 (s, 3 H), 0.89 (s, $3 \mathrm{H}) ;{ }^{13} \mathrm{C}$ NMR (75 MHz, $\left.\mathrm{CDCl}_{3}\right): \delta 179.4,169.7,165.4,164.0,140.8,132.0$, 121.4, 113.9, 99.9, 99.2, 89.7, 84.9, 78.4, 72.8, 72.5, 71.7, 70.1, 69.2, 66.4, 63.8, 61.1, 55.5, 49.5, 47.9, 46.3, 42.2, 37.1, 36.4, 35.8, 32.8, 32.4, 31.8, 31.6, 25.9, 20.6, 19.4, 13.4, 12.6; MALDI-MS m/z: $884.4\left(\mathrm{M}+\mathrm{Na}^{+}\right), 890.4\left(\mathrm{M}+\mathrm{K}^{+}\right)$; HRMS (MALDI) $\mathrm{m} / \mathrm{z}$ : 
884.4058 $\left(\mathrm{M}+\mathrm{Na}^{+}\right)$; Calcd for $\mathrm{C}_{44} \mathrm{H}_{63} \mathrm{NaNO}_{16}: 884.4039$.

\section{Preparation of compound 85}
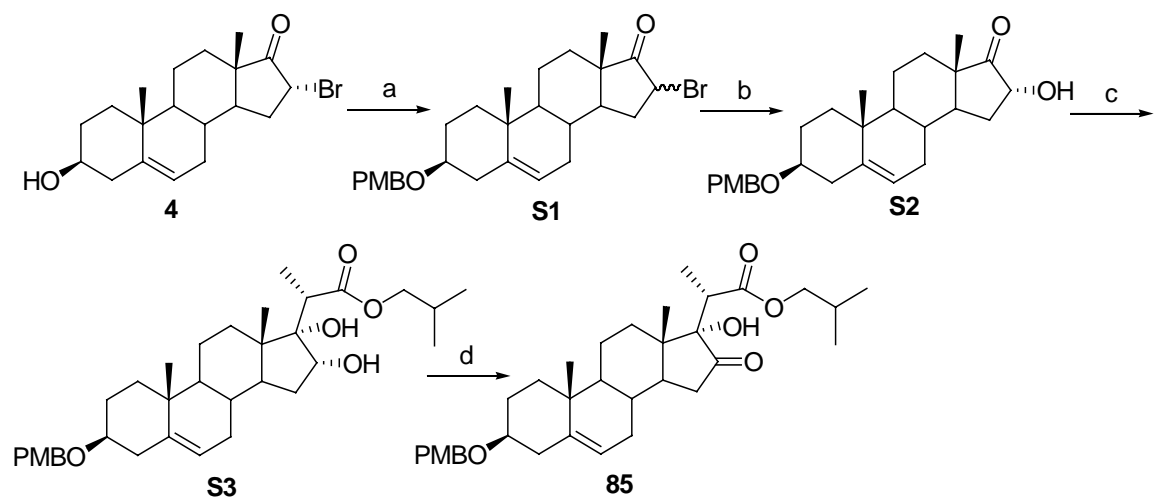

Reagents and conditions: a) PMB-imidate, 0.05 eq PPTS, $\mathrm{CH}_{2} \mathrm{Cl}_{2}$, rt, overnight, $95 \%$ recovered yield; b) 1.2 eq $\mathrm{NaOH}, \mathrm{DMF}-\mathrm{H}_{2} \mathrm{O}-\mathrm{CH}_{2} \mathrm{Cl}_{2}$ (v/v/v, 3:1:2), rt, 97\%; (c) i) i-Pr ${ }_{2} \mathrm{NH}, n$-BuLi, $-78{ }^{\circ} \mathrm{C}, 15 \mathrm{~min}$; ii) THF, $-78{ }^{\circ} \mathrm{C}, \mathrm{C}_{2} \mathrm{H}_{5} \mathrm{C}(\mathrm{O}) \mathrm{OCH}_{2} \mathrm{CH}\left(\mathrm{CH}_{3}\right)_{2}$, 0.5h; iii) S2, $-78^{\circ} \mathrm{C}, 73 \%$; (d) TPAP, $\mathrm{NMO}, \mathrm{CH}_{2} \mathrm{Cl}_{2}, 4 \AA \mathrm{MS}$, rt, overnight, 92\%.

Compound 85: $[\alpha]^{15}=-118.4\left(c\right.$ 1.0, $\left.\mathrm{CHCl}_{3}\right) ;{ }^{1} \mathrm{H}$ NMR (300 MHz, $\left.\mathrm{CDCl}_{3}\right): \delta 7.27$ and 6.88 (AB, 4 H), 5.35 (m, $1 \mathrm{H}), 4.50$ (s, $2 \mathrm{H}), 4.03$ and 3.92 (2 AB, $4 \mathrm{H}), 3.81$ (s, $3 \mathrm{H})$, 3.28-3.21 (m, 1 H), 2.59 (q, $J=7.2$ Hz, 1 H), 1.29 (d, $J=7.2$ Hz, 3 H), 1.04 (s, 3 H), 0.98 (d, $J=6.6 \mathrm{~Hz}, 6 \mathrm{H}), 0.80$ (s, $3 \mathrm{H}) ;{ }^{13} \mathrm{C}$ NMR $\left(75 \mathrm{MHz}, \mathrm{CDCl}_{3}\right): \delta 216.4,178.9,159.0$, 141.0, 131.0, 129.1, 120.8, 113.7, 82.5, 78.0, 71.1, 69.6, 55.3, 49.3, 45.1, 45.0, 39.1, 38.5, 36.9, 35.8, 31.8, 31.0, 30.3, 28.4, 27.6, 20.1, 19.4, 19.1, 19.0, 13.8, 12.7; ESIMS m/z: $553.1\left(\mathrm{M}+\mathrm{H}^{+}\right)$; HRMS (ESI) $\mathrm{m} / \mathrm{z}$ : $575.3342\left(\mathrm{M}+\mathrm{Na}^{+}\right)$; Calcd for $\mathrm{C}_{34} \mathrm{H}_{48} \mathrm{O}_{6} \mathrm{Na}$ : 575.3367. 


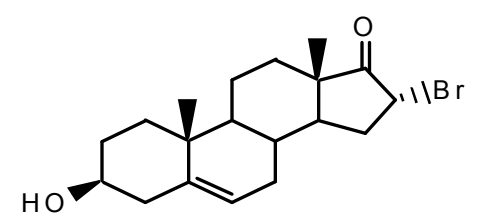

$\mathrm{C}_{19} \mathrm{H}_{27} \mathrm{BrO}_{2}$

Exact Mass: 366.1194

Mol. W t.: 367.3205

C, 62.13; H, 7.41; B r, 21.75; O, 8.71

$\mathrm{CDCl}_{3}$ 
$\mathrm{Cls}_{\mathrm{b}+62}$ in cac13

Pulse Sequencet :20ei

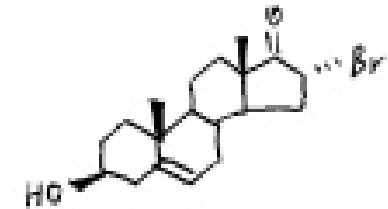

SBFI I-1-1 (62)

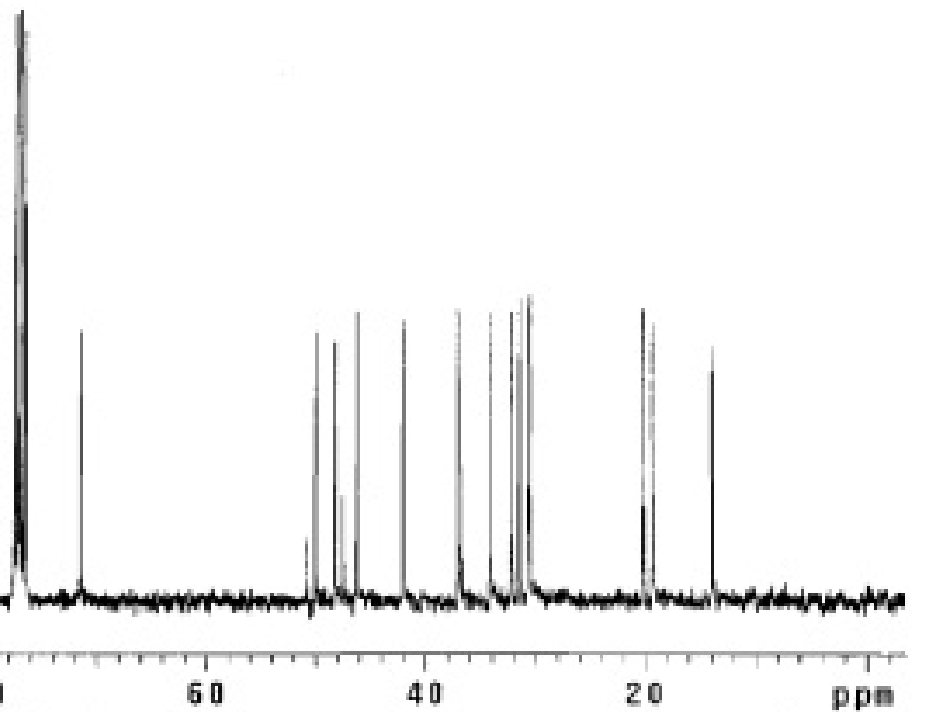

${ }^{13} \mathrm{CNMR}$ spectrum $(75 \mathrm{MHz})$ of 2 in $\mathrm{CDCl}_{3}$ 


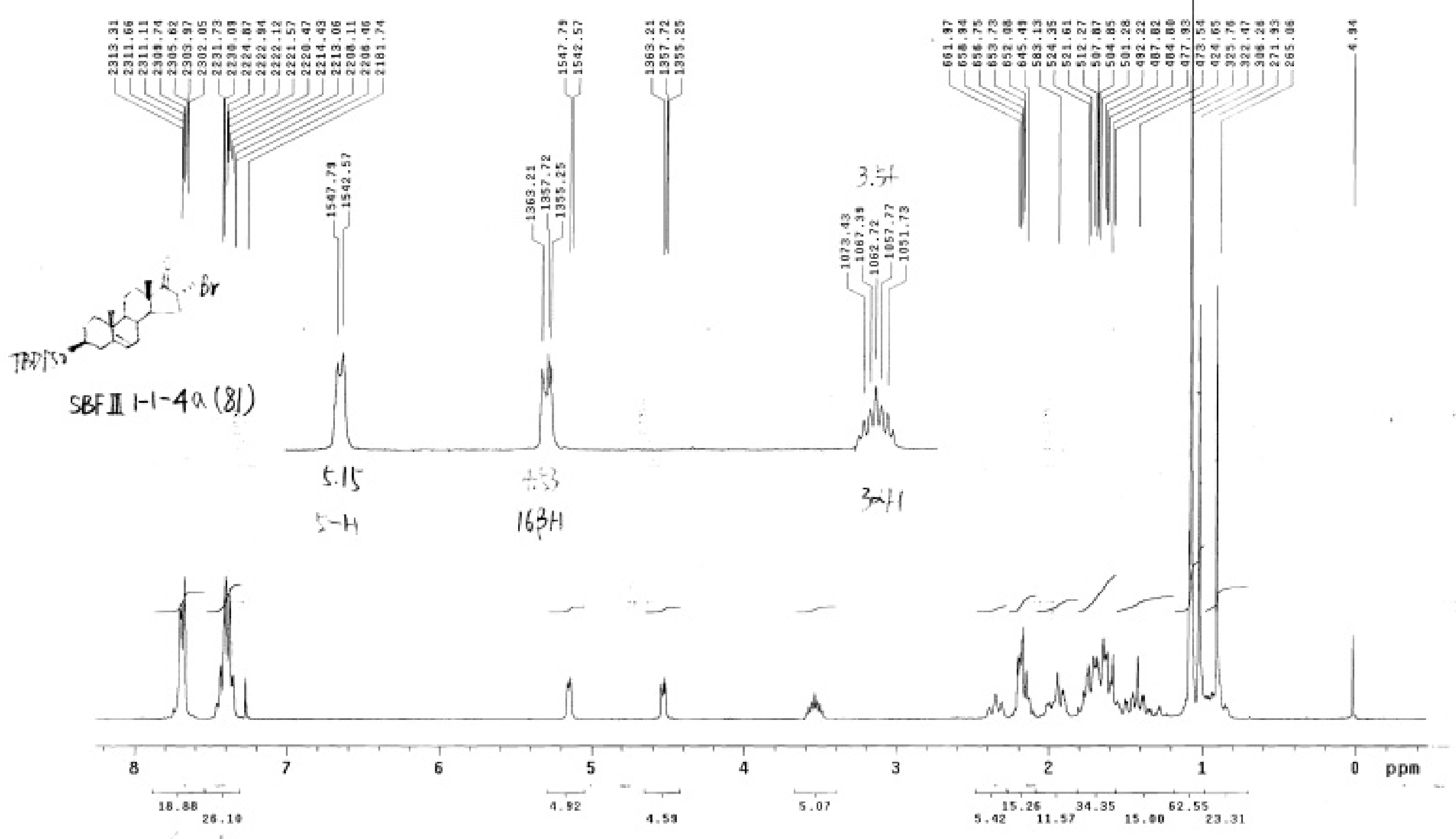

${ }^{1} \mathrm{H}$ NMR spectrum (300 MHz) of $16 \alpha$-isomer 3 in $\mathrm{CDCl}_{3}$ 
Pulse Sequence: s2pul

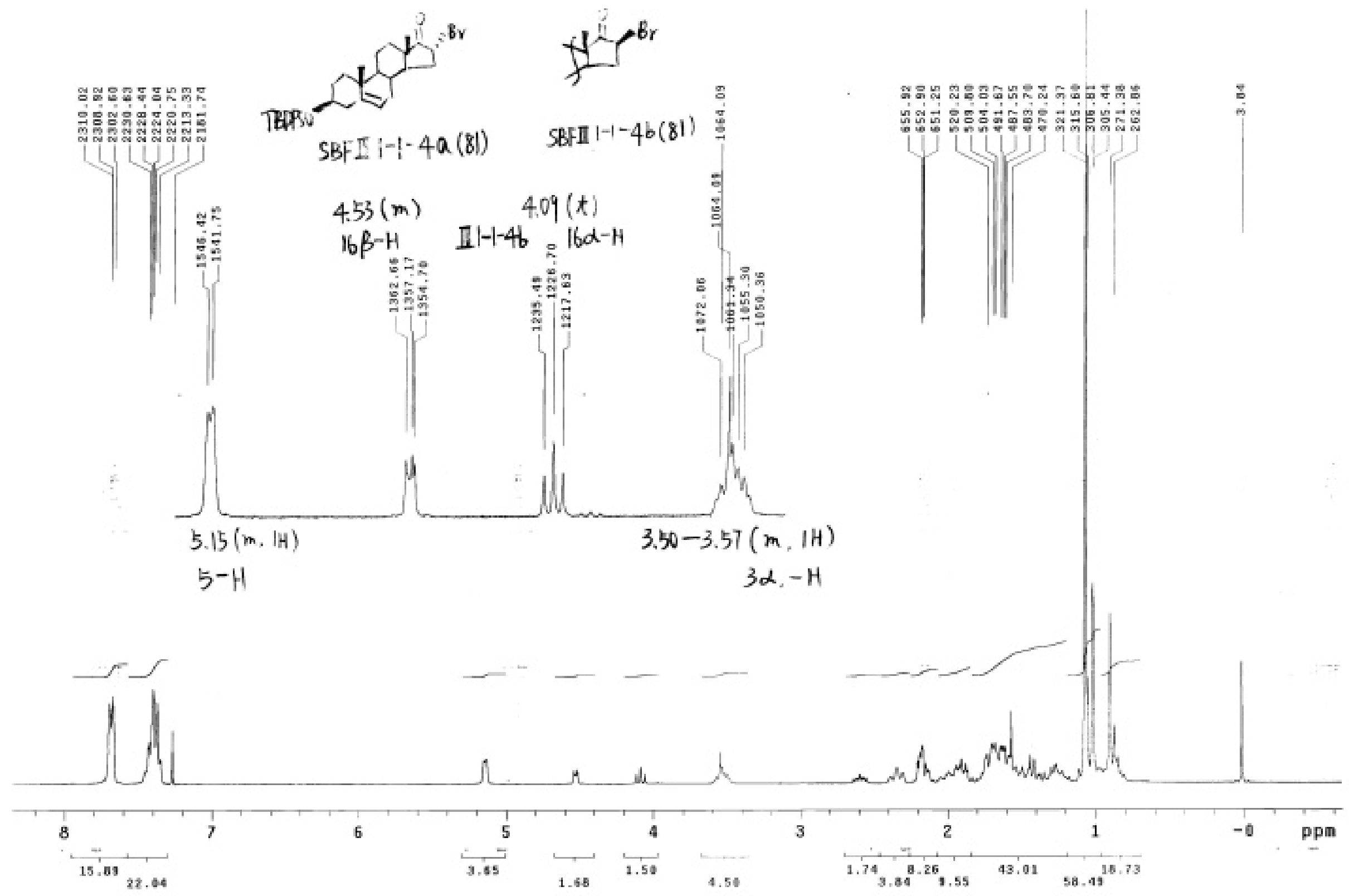

${ }^{1} \mathrm{H}$ NMR spectrum $(300 \mathrm{MHz})$ of $16 \alpha$ and $16 \beta$ mixture of 3 in $\mathrm{CDCl}_{3}$ 


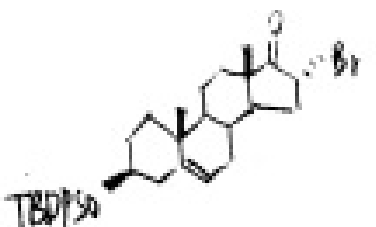

SBFIII-1-4a (81)

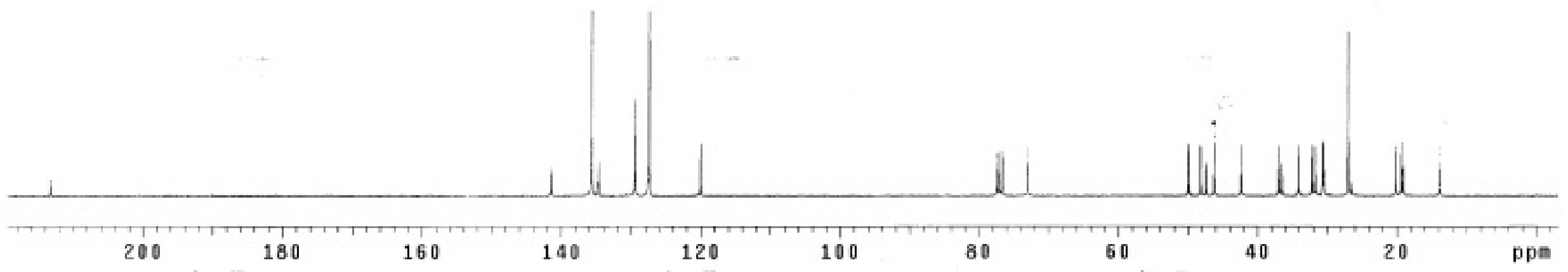




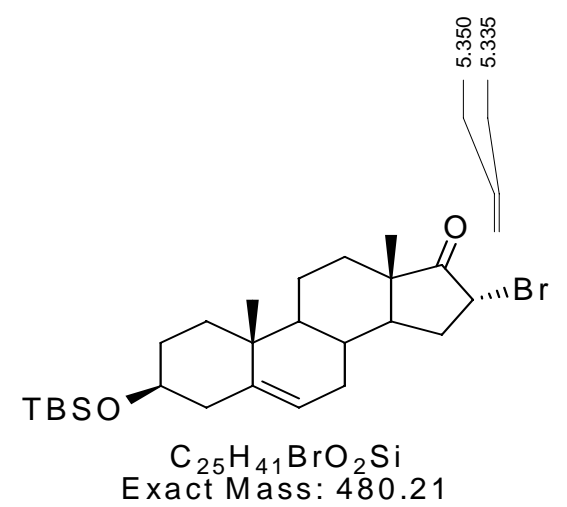

MolWt: 481.58

C, $62.35 ; \mathrm{H}, 8.58 ; \mathrm{Br}, 16.59 ; \mathrm{O}, 6.64 ; \mathrm{Si}, 5.83$

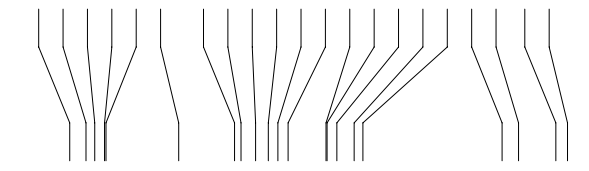

$\mathrm{CDCl}_{3}$

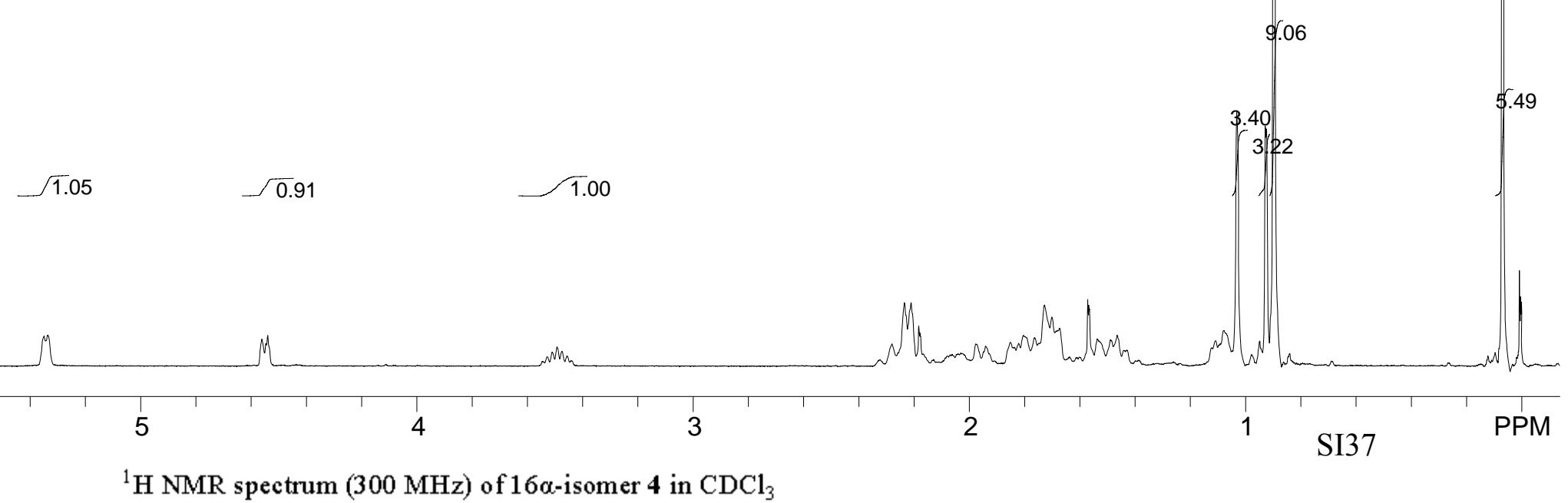




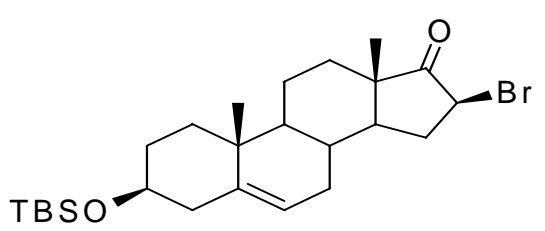

$\mathrm{C}_{25} \mathrm{H}_{41} \mathrm{BrO}_{2} \mathrm{Si}$

Exact Mass:
Mol. Wt.: 481

Mol. Wt.: 481.58

C, $62.35 ; \mathrm{H}, 8.58 ; \mathrm{Br}, 16.59 ; \mathrm{O}, 6.64 ; \mathrm{Si}, 5.83$

$\mathrm{CDCl}_{3}$

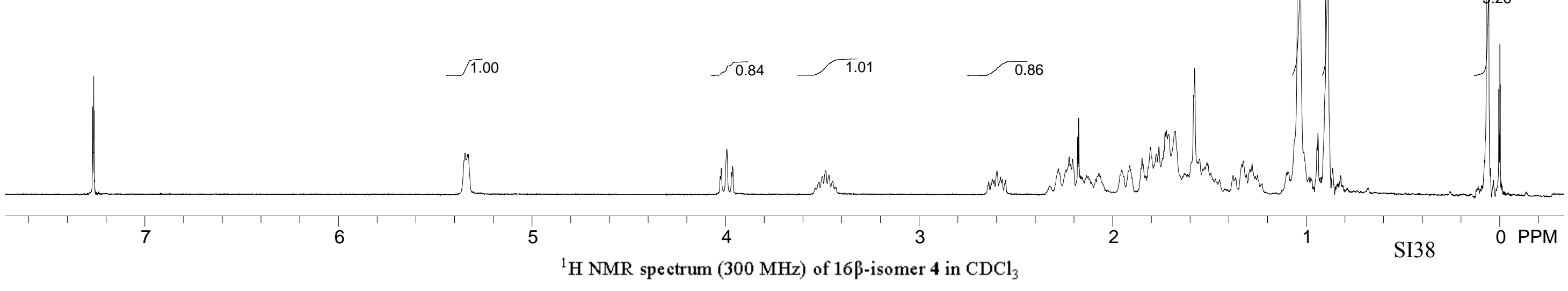




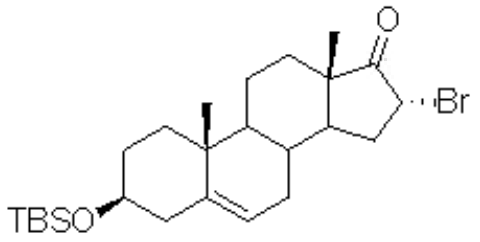

$\mathrm{C}_{25} \mathrm{H}_{41} \mathrm{BrO}_{2} \mathrm{Si}$

Exact Mass: 480.21

Mol Wt: 48158

C. $62.35 ; \mathrm{H}, 8.58 ; \mathrm{Br}, 16.59 ; 0,6.64 ; \mathrm{Si}, 5.83$

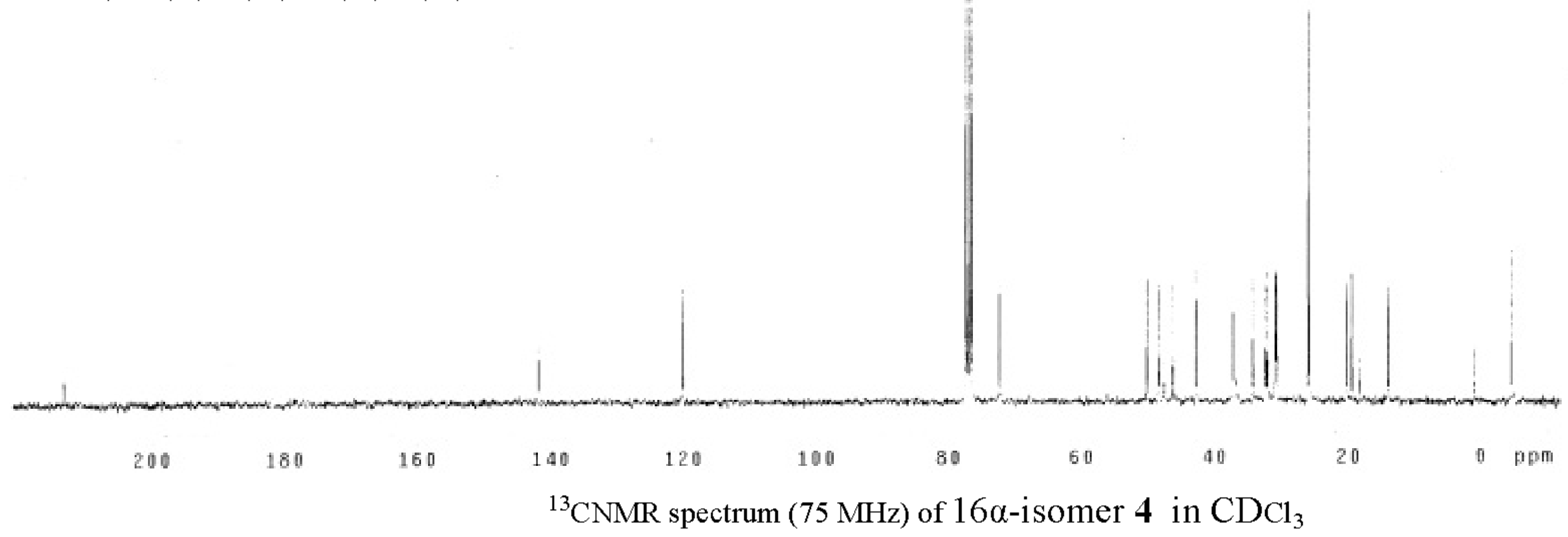

SI39 

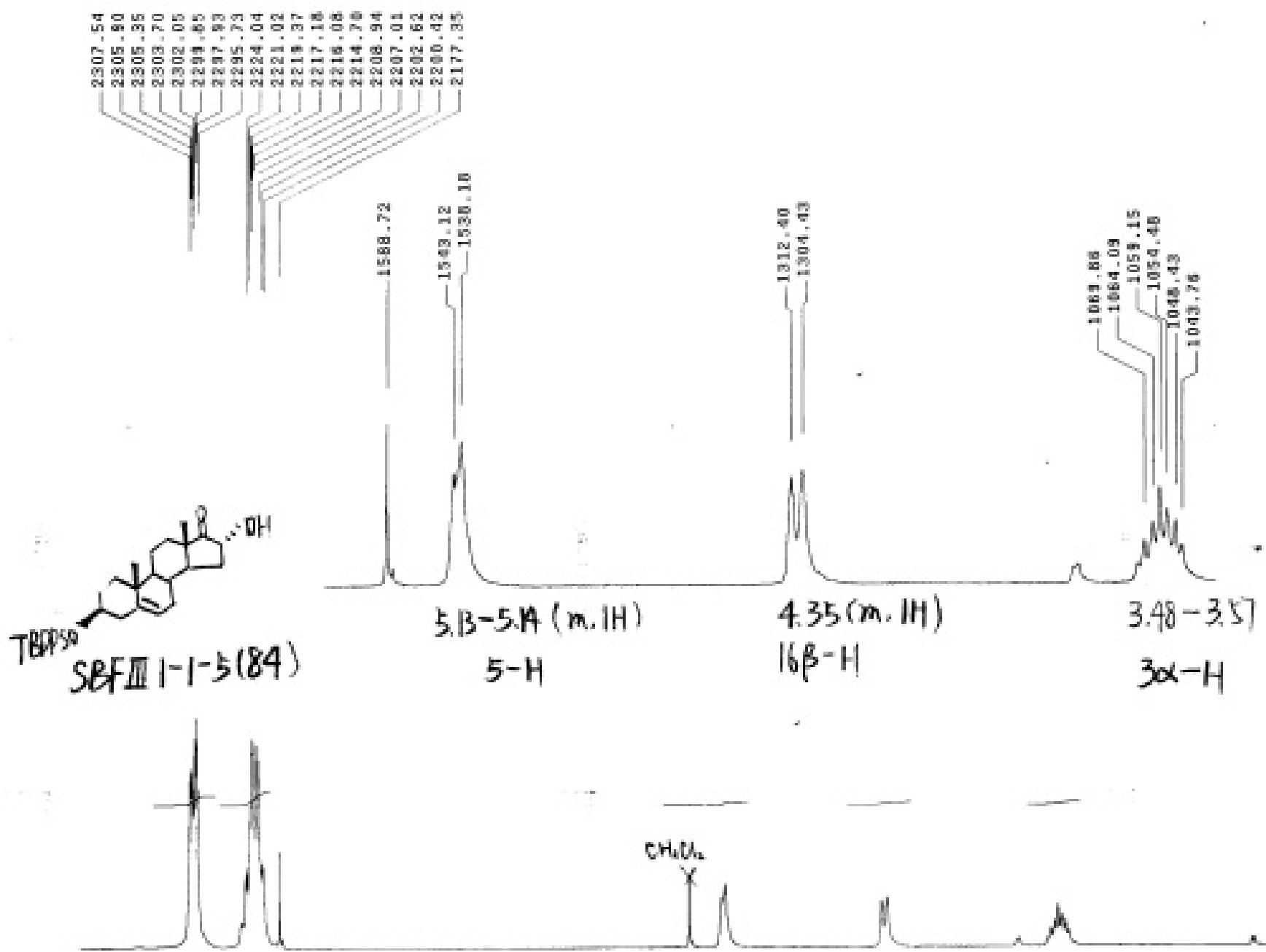

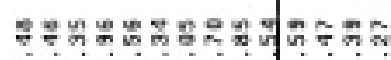

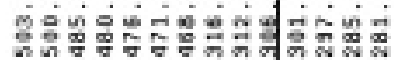

(1) 
C19

Pulse sequence: srpul
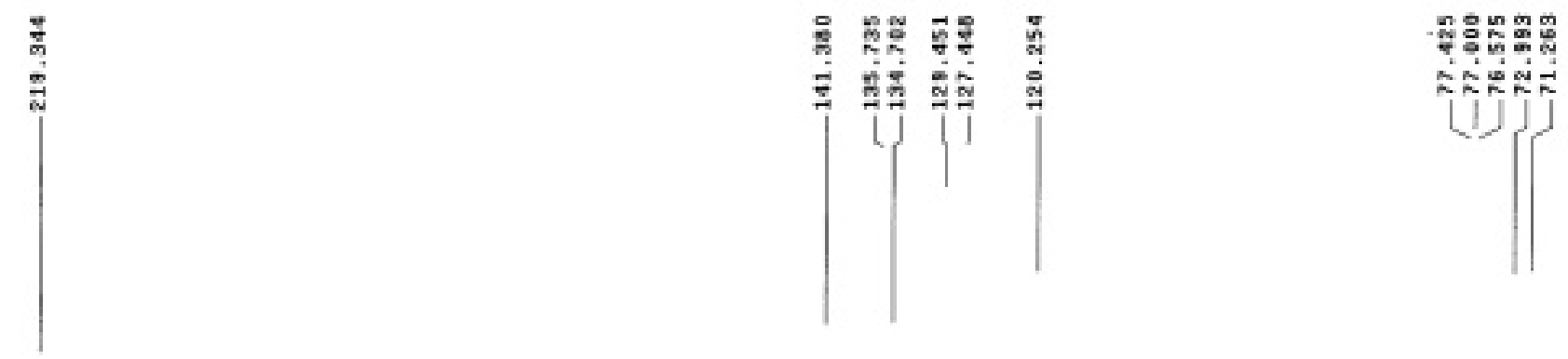

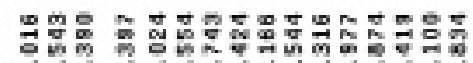

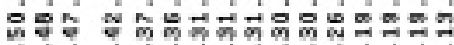

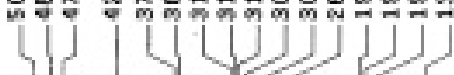

(1)
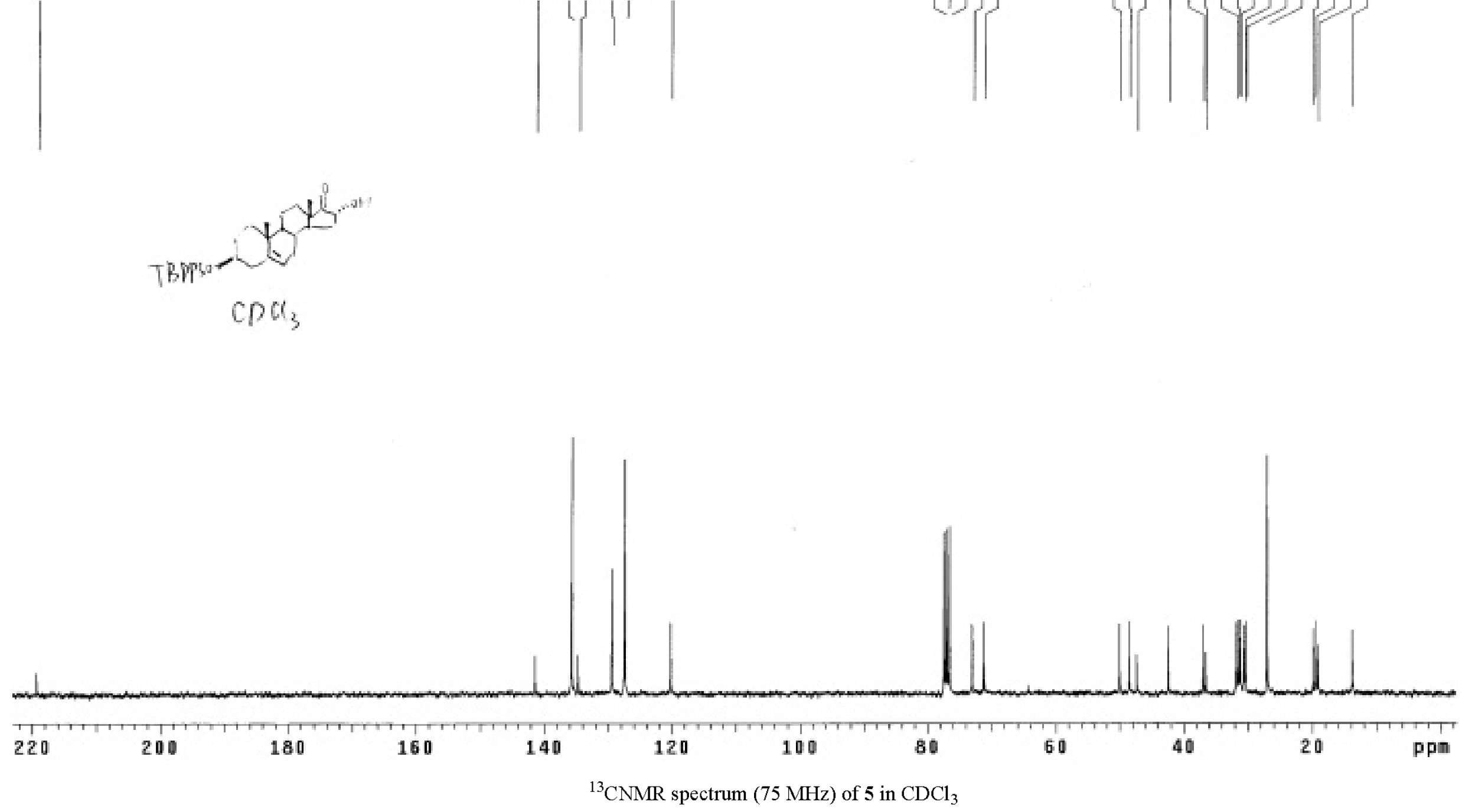


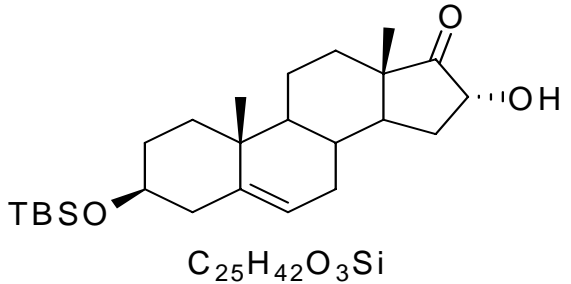

Exact Mass: 418.29

Mol. Wt.: 418.68

C, $71.72 ; \mathrm{H}, 10.11 ; \mathrm{O}, 11.46 ; \mathrm{Si}, 6.71$

$$
\mathrm{CDCl}_{3}
$$

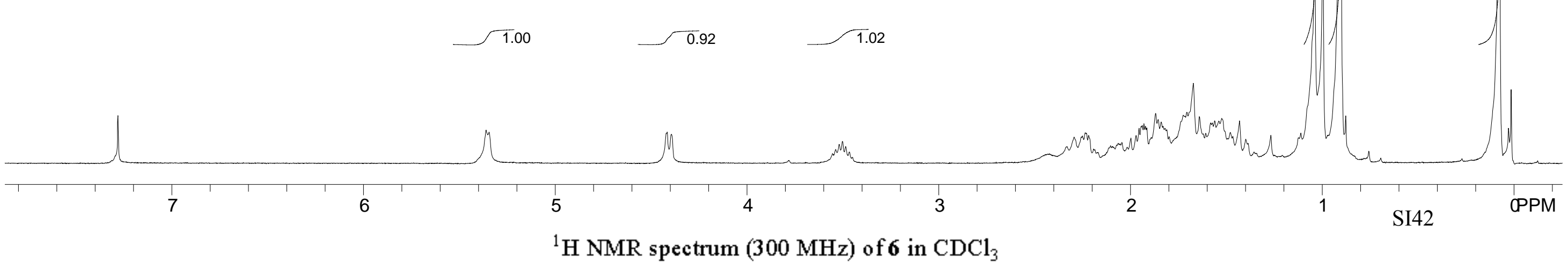



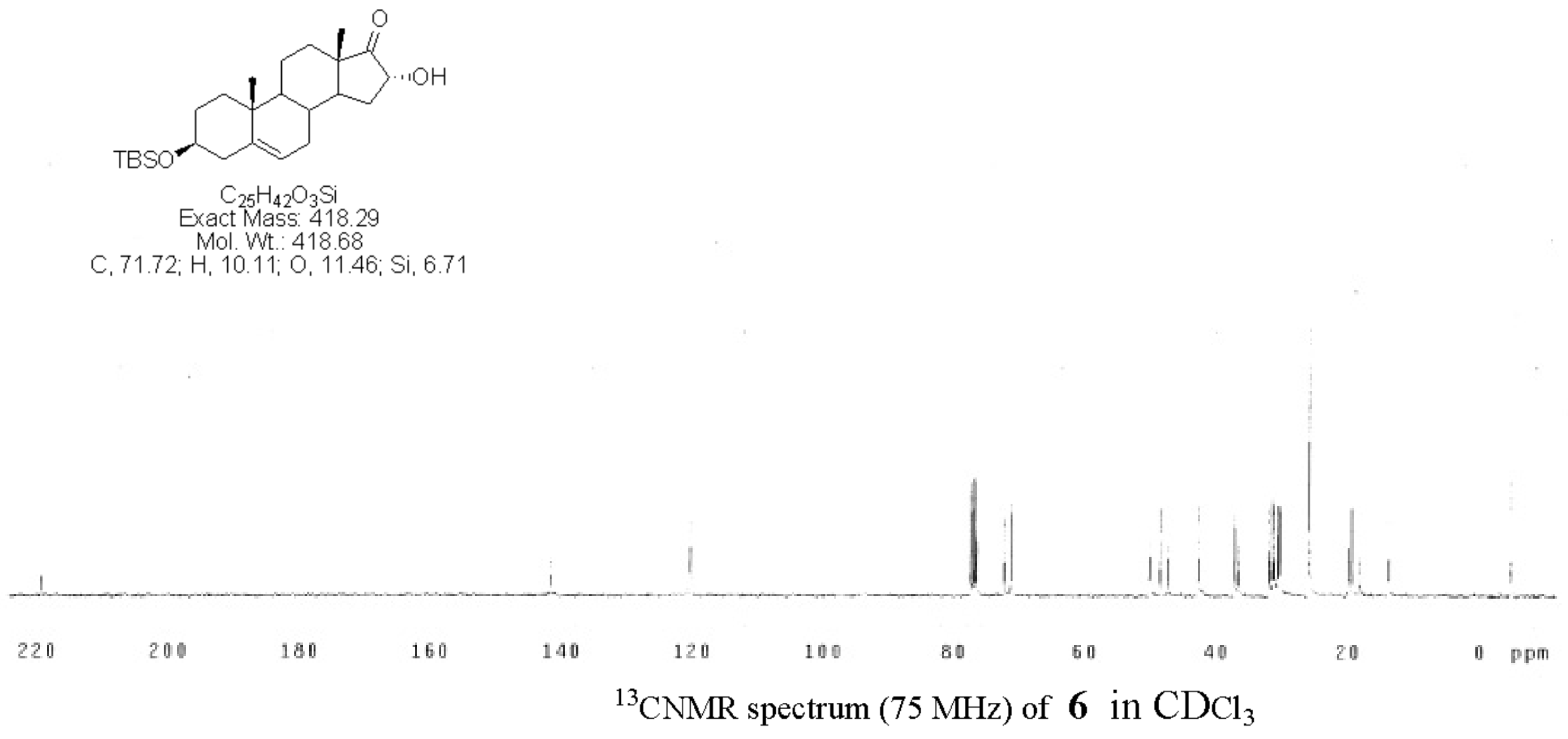

SI43 

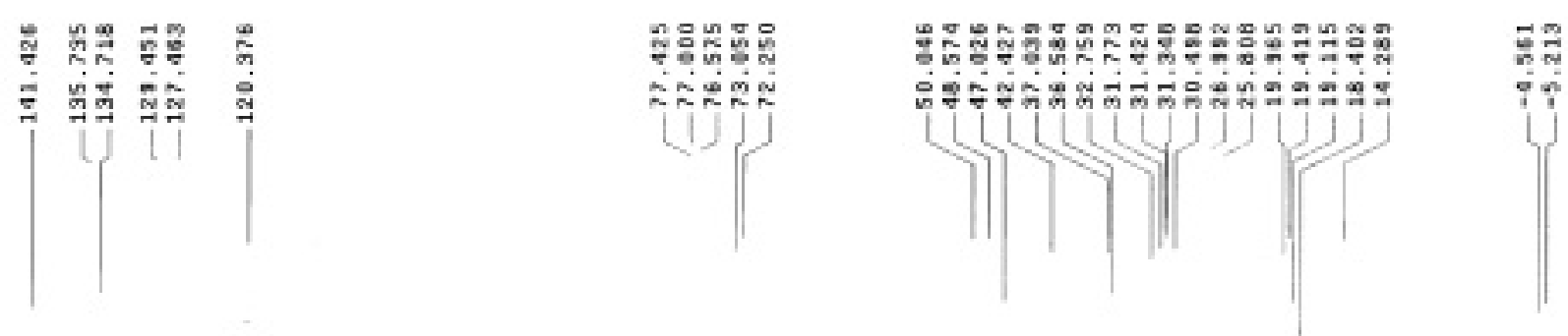

Sbf III-1-6 (90)

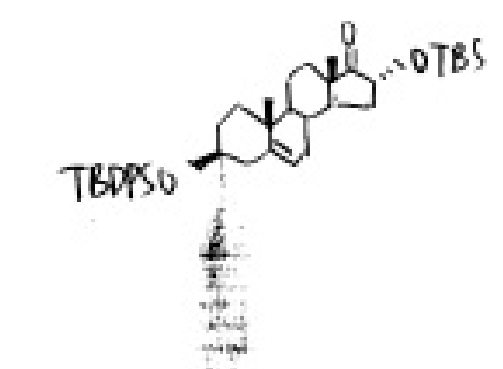

${ }^{13} \mathrm{CNMR}$ spectrum $(75 \mathrm{MHz})$ of 7 in $\mathrm{CDCl}_{3}$ 


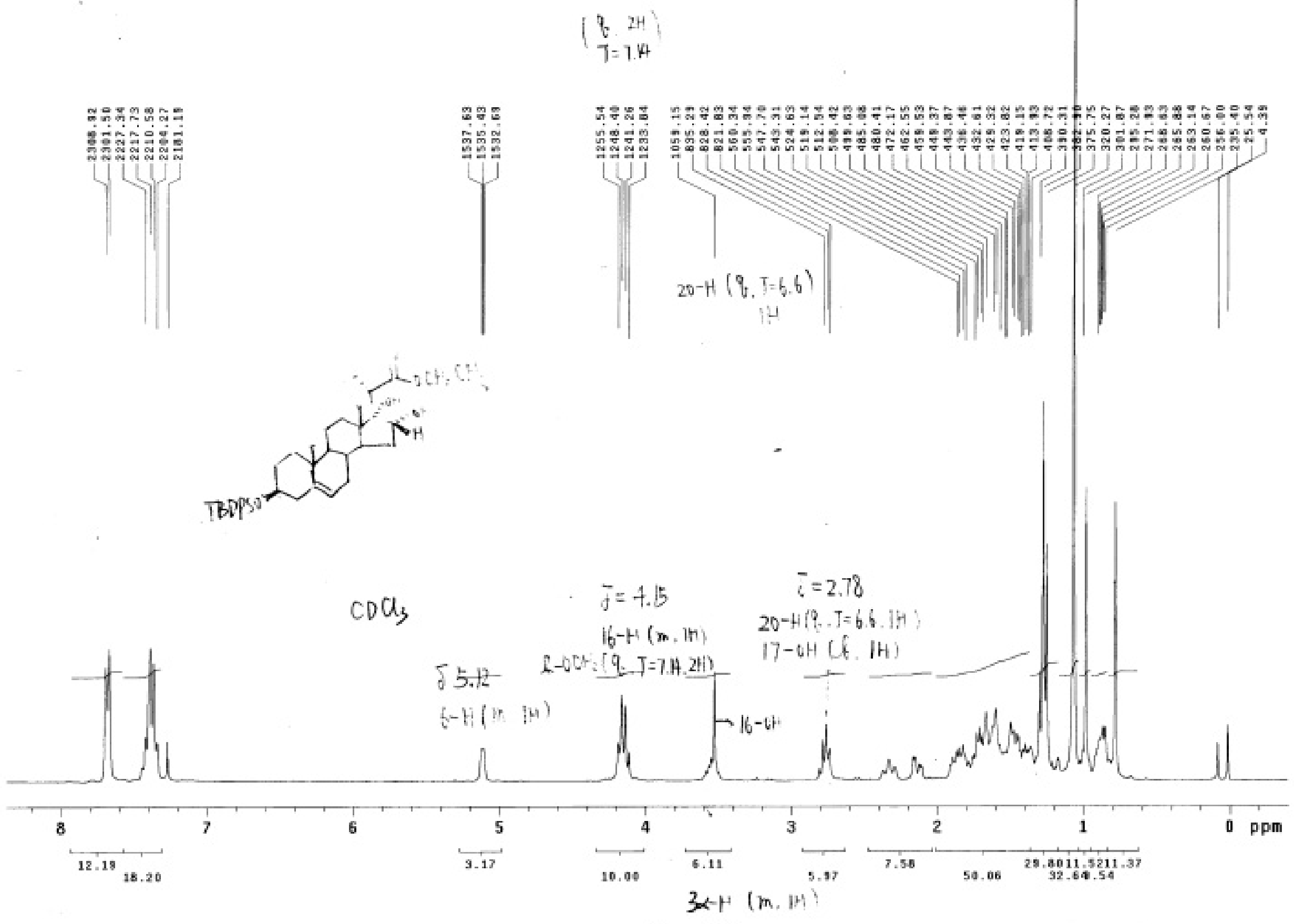

${ }^{1} \mathrm{H}$ NMR spectrum (300 $\mathrm{MHz}_{2}$, 8 8a in $\mathrm{CDCl}_{3}$ 

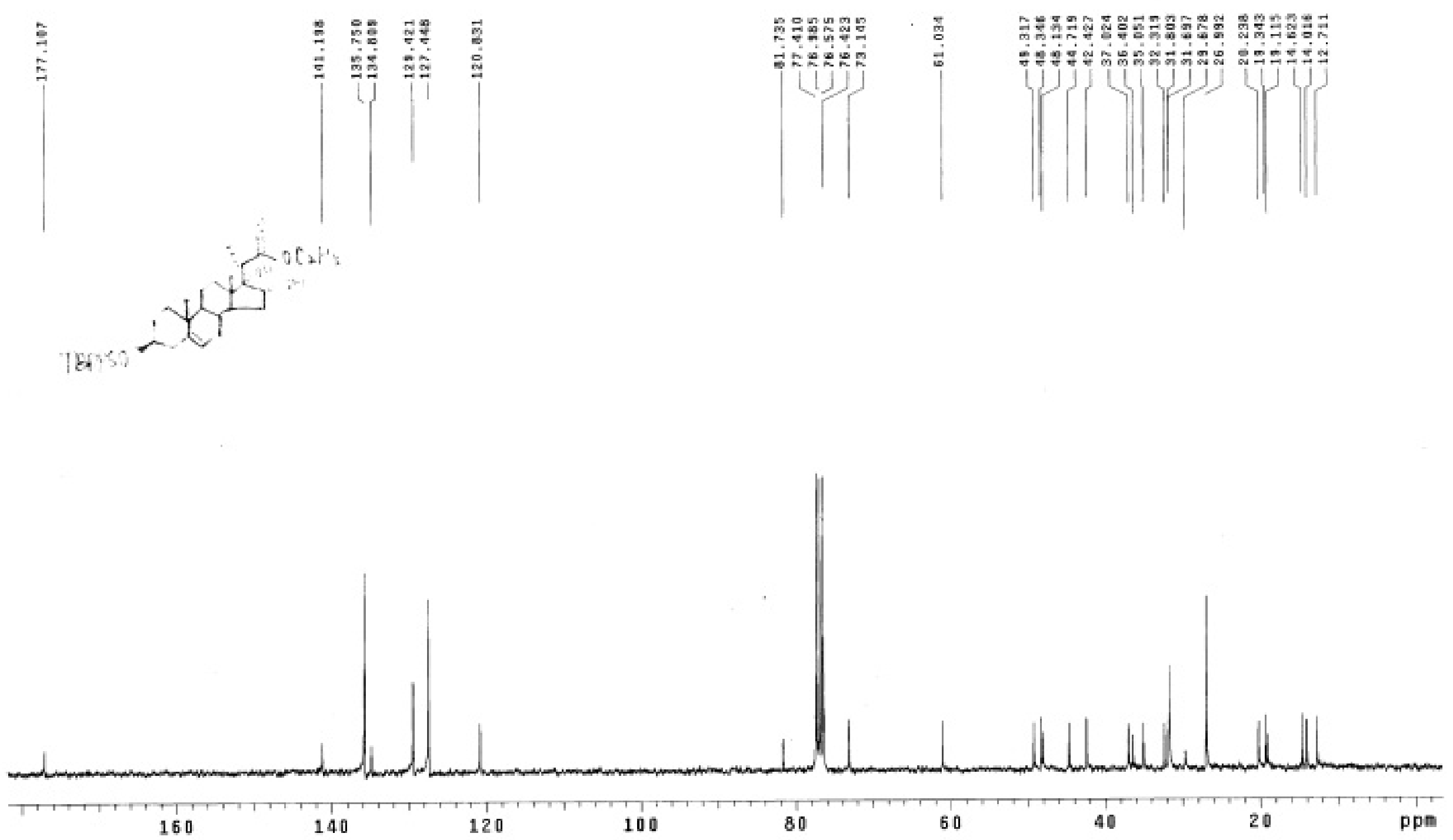

${ }^{13} \mathrm{CNMR}$ spectrum $\left(75 \mathrm{MHz}\right.$ ) of $\mathbf{8 a}$ in $\mathrm{CDCl}_{3}$ 


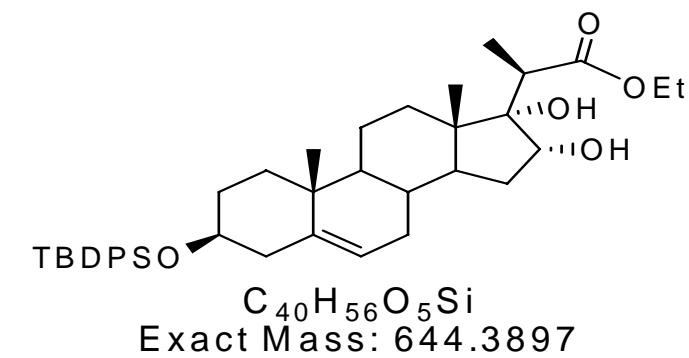

Exact Mass: 644.3897

C , $74.49 ; \mathrm{H}, 8.75 ; \mathrm{O}, 12.40 ; \mathrm{Si}, 4.35$

$\mathrm{CDCl}_{3}$

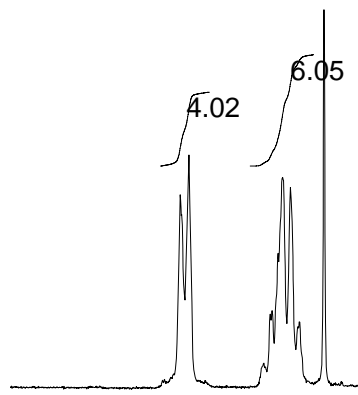




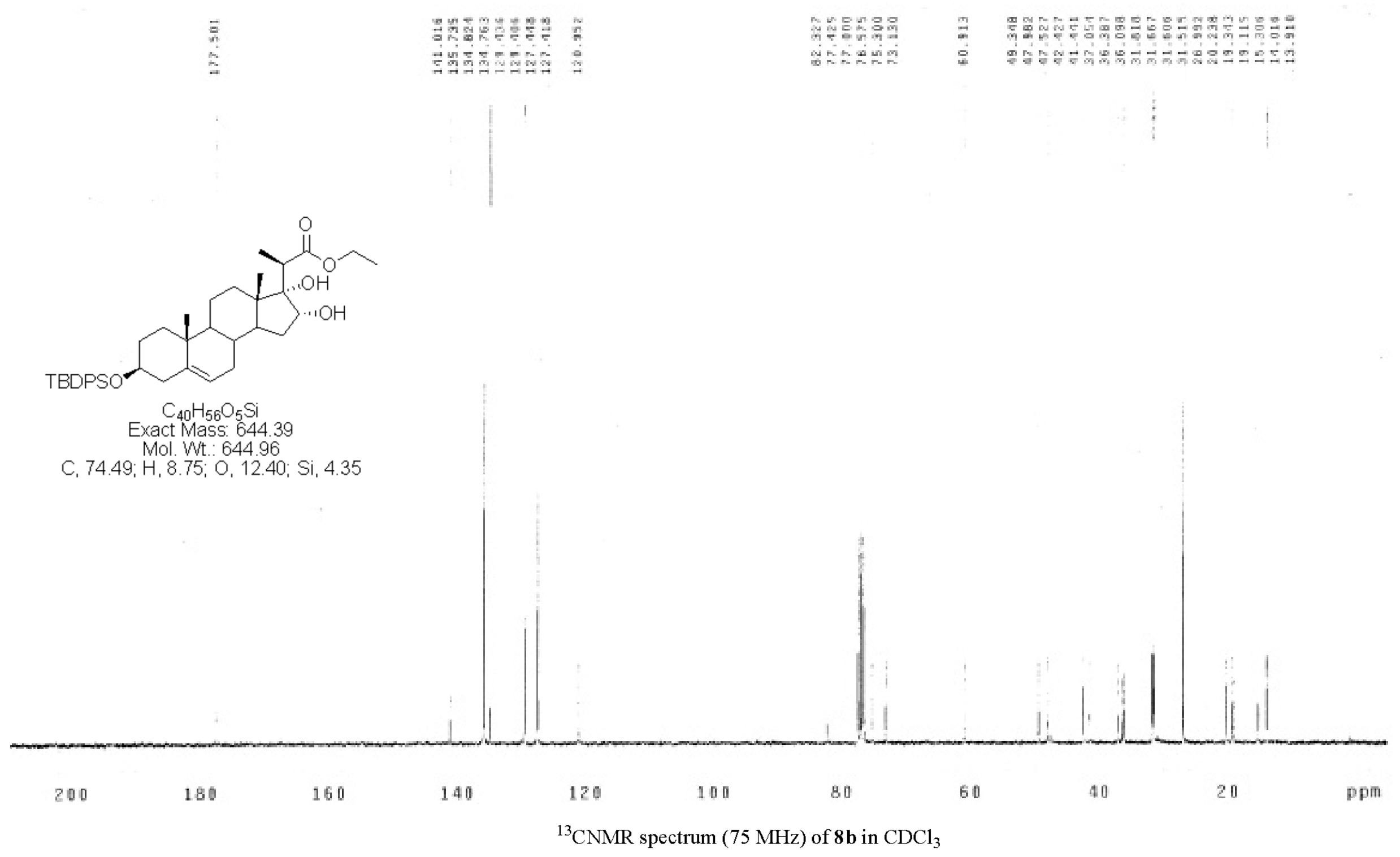

SI49 

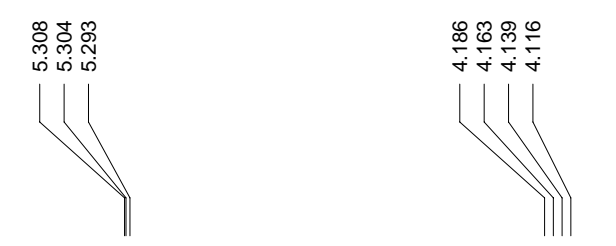

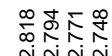

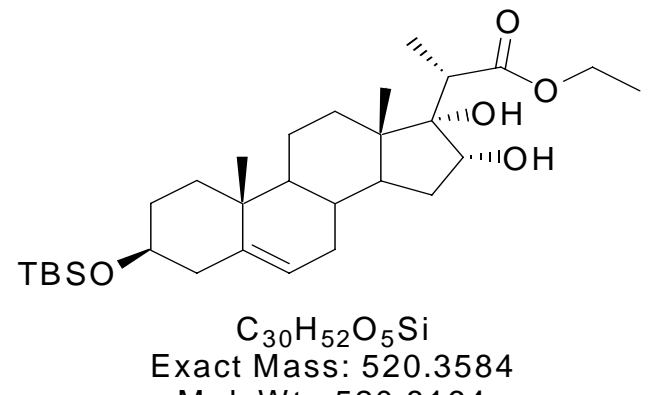

Mol. W

C, 69.18; H, 10.06; O, 15.36; Si, 5.39 


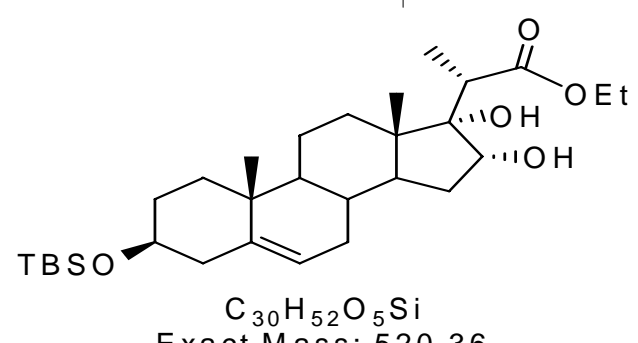

Exact Mass: 520.36

Mol. Wt.: 520.82

C , $69.18 ; \mathrm{H}, 10.06 ; 0,15.36 ; \mathrm{Si}, 5.39$ $\mathrm{CDCl}_{3}$

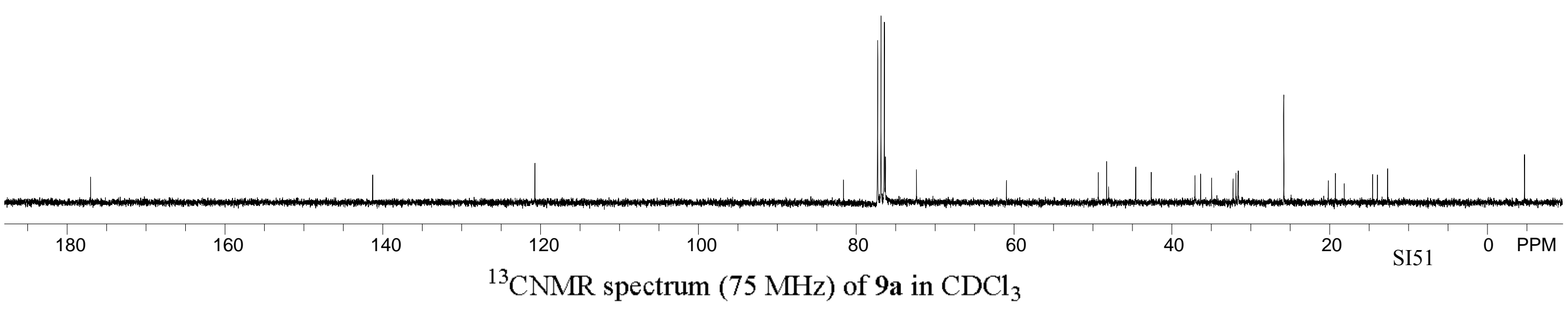




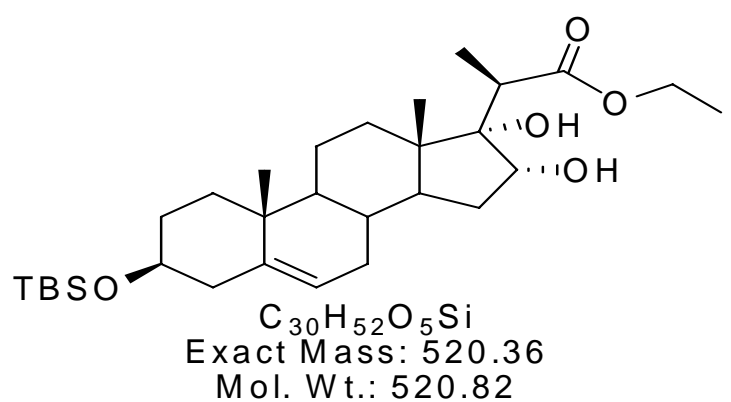

C, 69.18; H, 10.06; O, 15.36; Si, 5.39

$\mathrm{CDCl}_{3}$ 


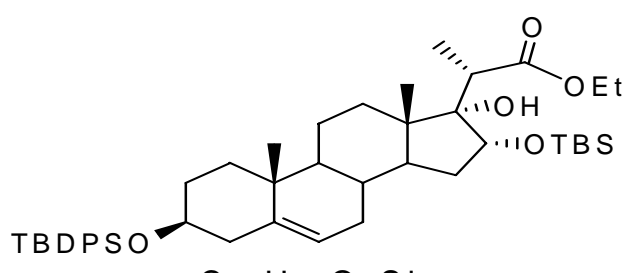

$\mathrm{C}_{46} \mathrm{H}_{70} \mathrm{O}_{5} \mathrm{Si}_{2}$

Exact Mass: 758.4762

Mol. W t.: 759.216

C, $72.77 ; \mathrm{H}, 9.29 ; \mathrm{O}, 10.54 ; \mathrm{Si}, 7.40$

$\mathrm{CDCl}_{3}$

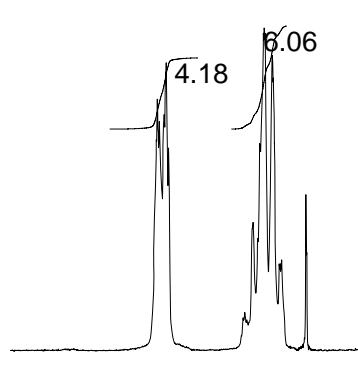

1.00

8

7

6

5

4

3

2

${ }^{1} \mathrm{H}$ NMR spectrum $(300 \mathrm{MHz})$ of $10 a$ in $\mathrm{CDCl}_{3}$

SI54

PPM 


\begin{tabular}{|c|c|c|c|}
\hline 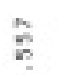 & : & สำำ & 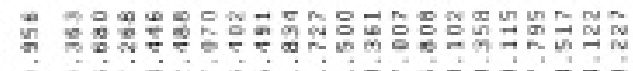 \\
\hline$\cong$ & $\Xi$ 踏 & DERED & 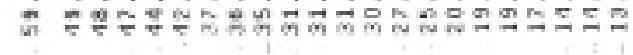 \\
\hline
\end{tabular}

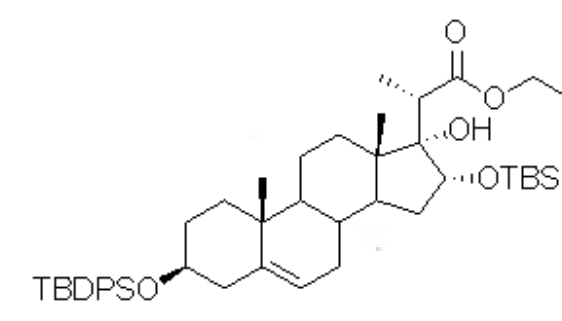

$\mathrm{C}_{46} \mathrm{H}_{70} \mathrm{O}_{5} \mathrm{Si}_{2}$

Mol. Wt.: 759.22

C. $72.77 ; \mathrm{H}, 9.29 ; 0,10.54$; Si, 7.40

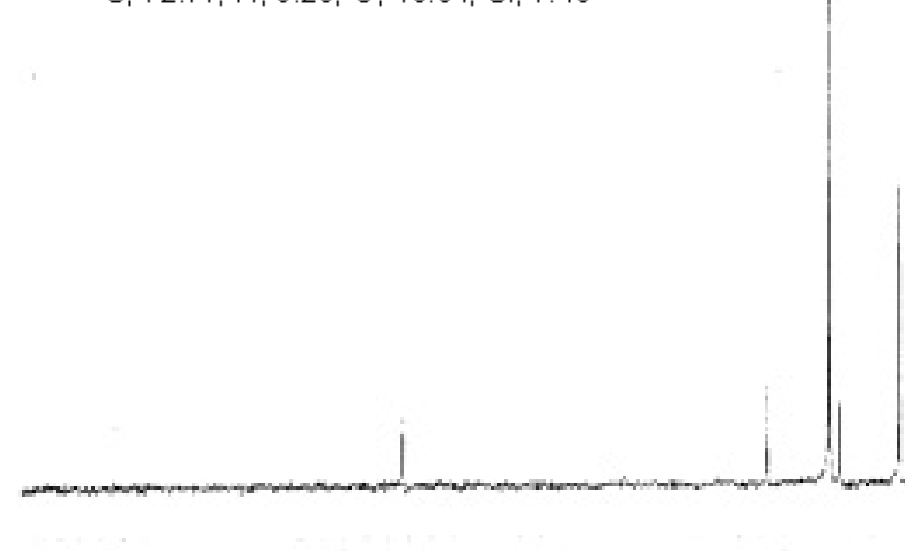

200

180

160

140

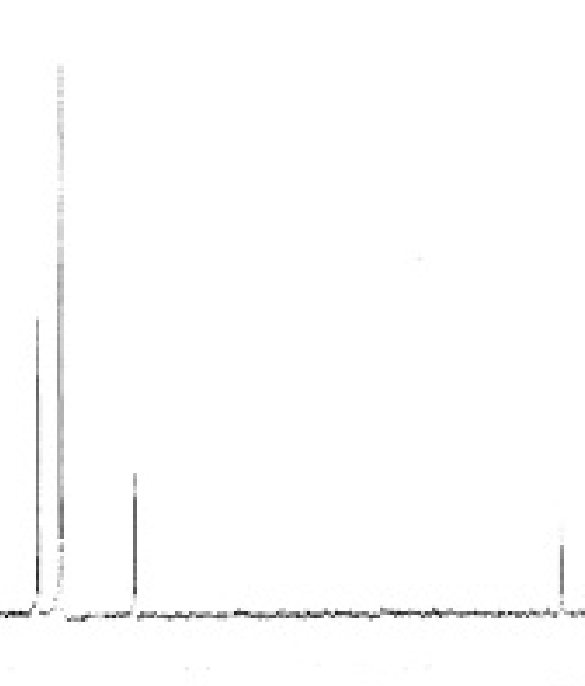

${ }^{13} \mathrm{CNMR}$ spectrum $\left(75 \mathrm{MHz}\right.$ ) of $10 \mathrm{a}$ in $\mathrm{CDCl}_{3}$ 


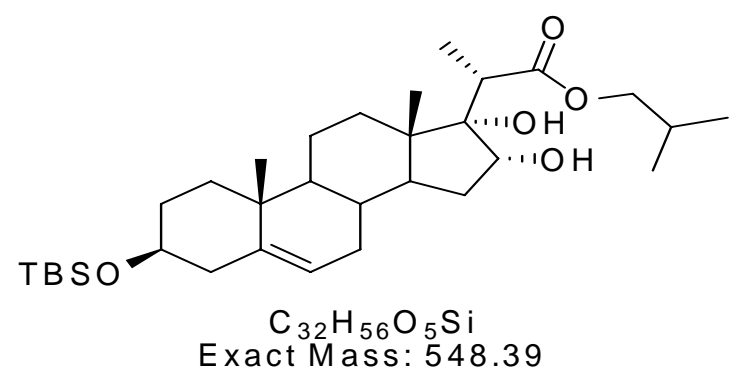

xact Mass: 548.39

C, $70.02 ; \mathrm{H}, 10.28 ; 0,14.57 ; \mathrm{Si}, 5.12$

$$
\mathrm{CDCl}_{3}
$$




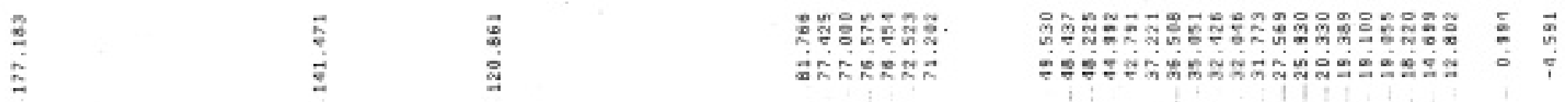

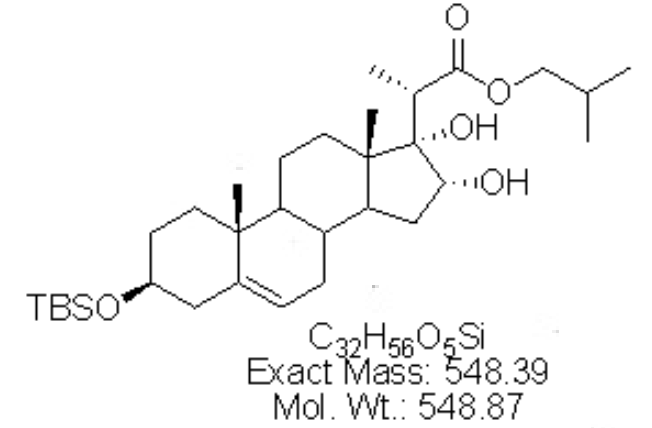

C. $70.02 ; \mathrm{H}, 10.28 ; 0,14.57 ; \mathrm{Si}, 5.12$

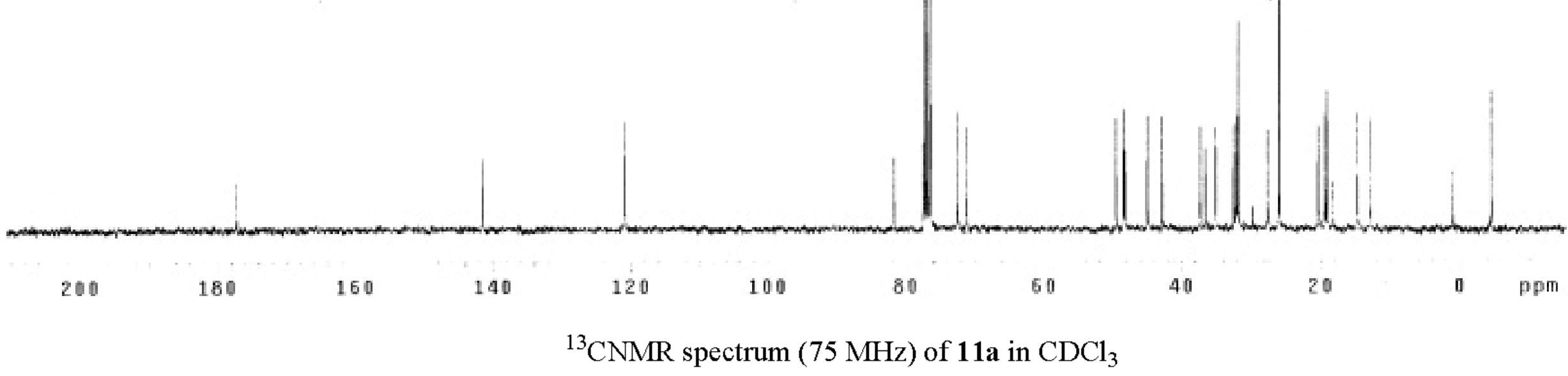



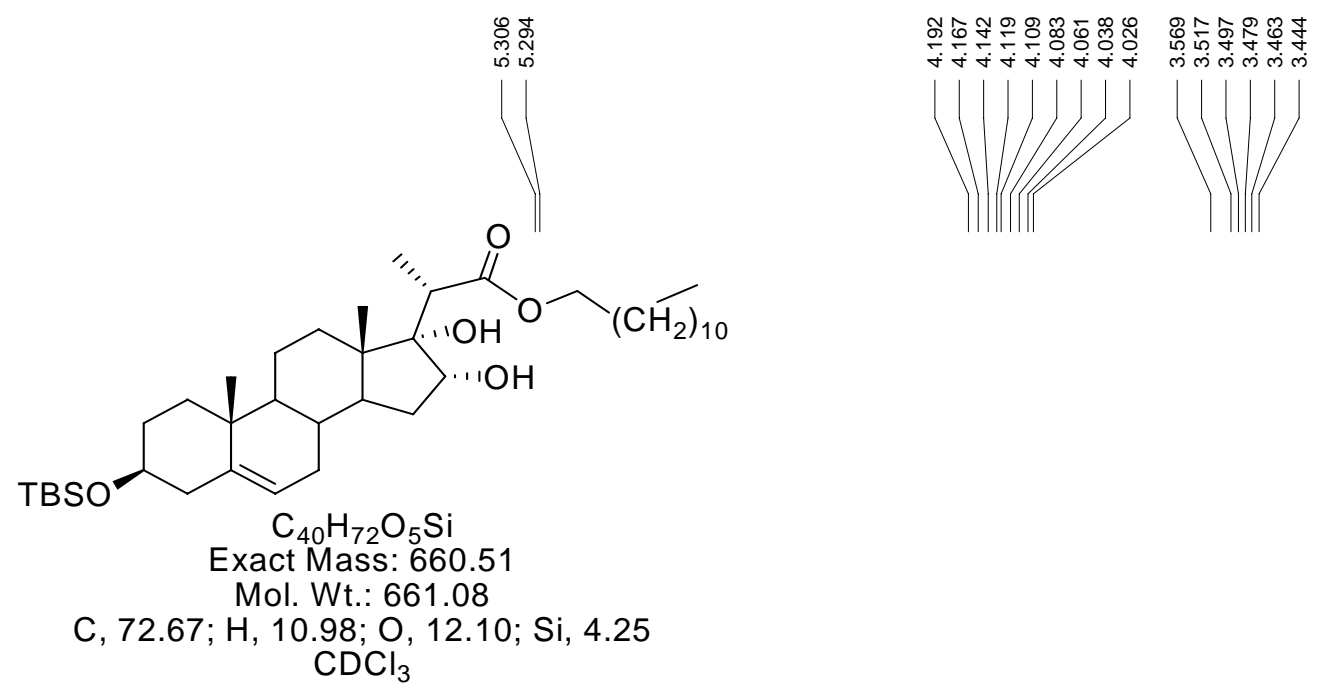

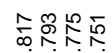

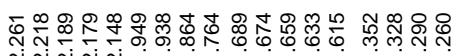

$$
\mathrm{CDCl}_{3}
$$

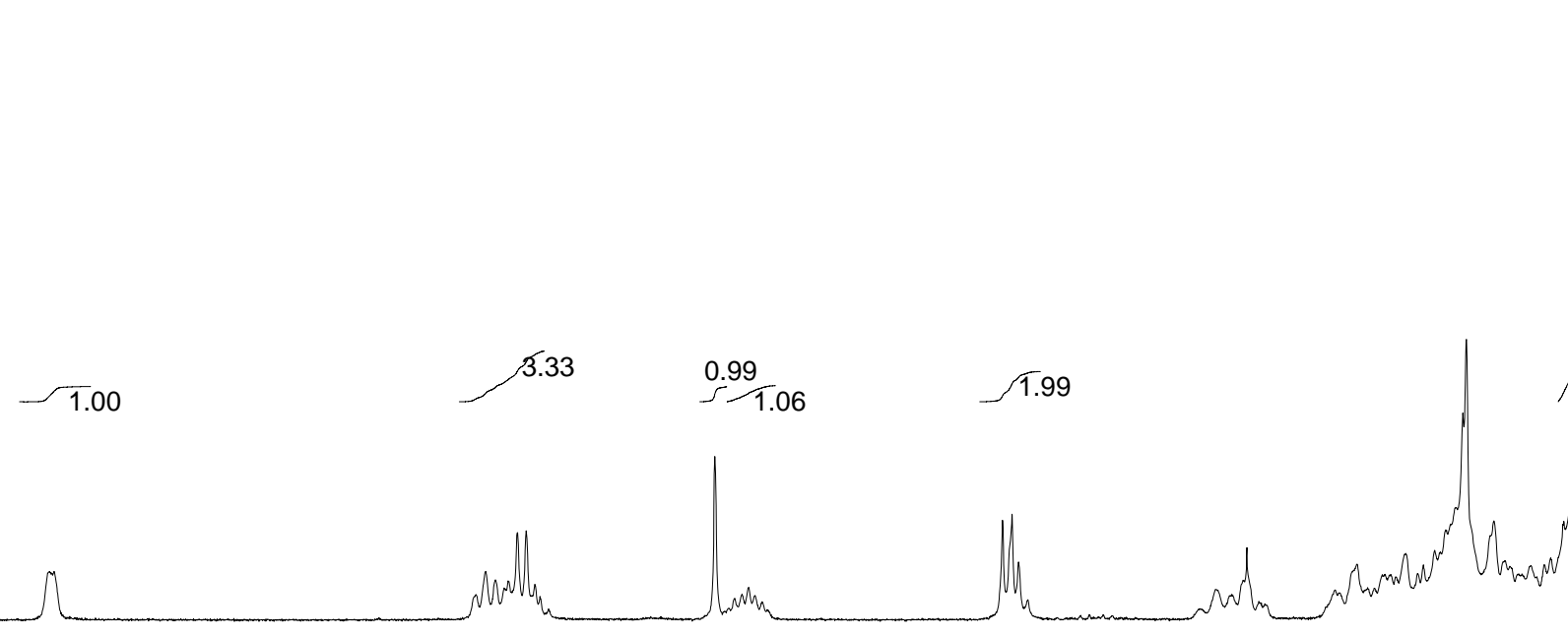




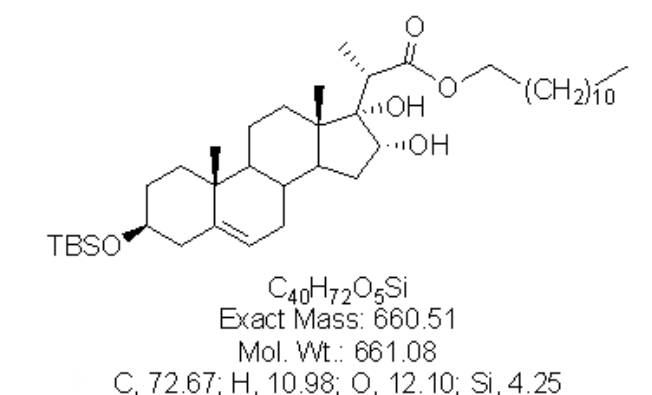

C. $72.67 ; \mathrm{H}, 10.98 ; 0,12.10 ; \mathrm{Si}, 4.25$

$\mathrm{CDCl}_{3}$

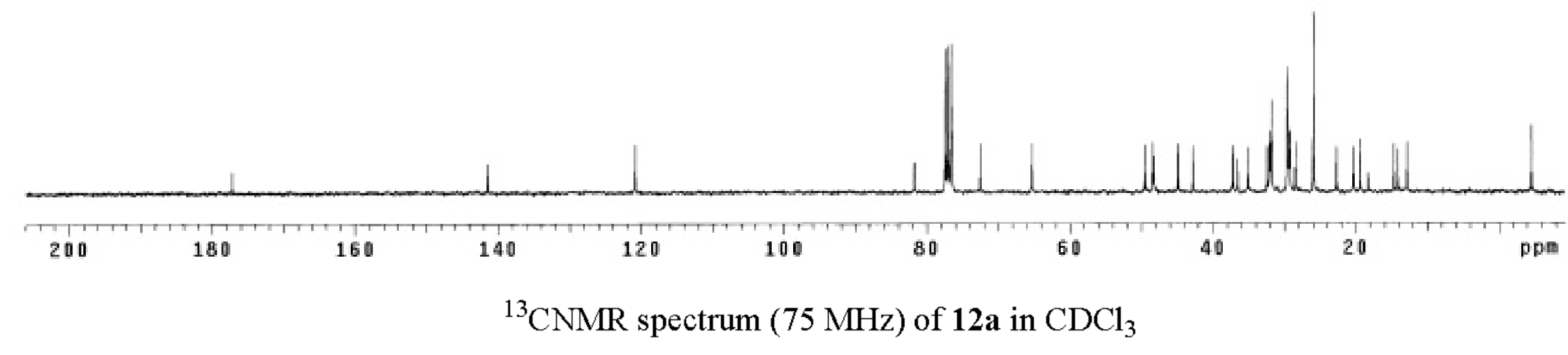



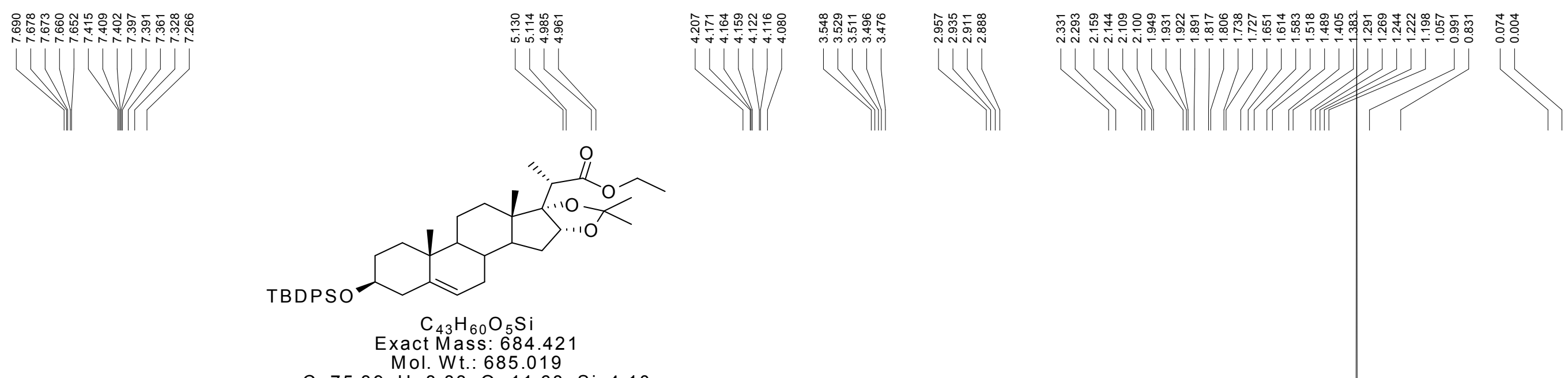

Mol. Wt. 685.019

C, $75.39 ; \mathrm{H}, 8.83 ; \mathrm{O}, 11.68 ; \mathrm{Si}, 4.10$

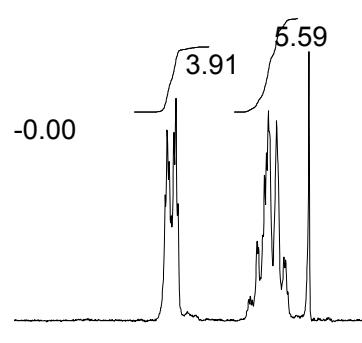

0.97

$-1.02^{1.03} 0.95$

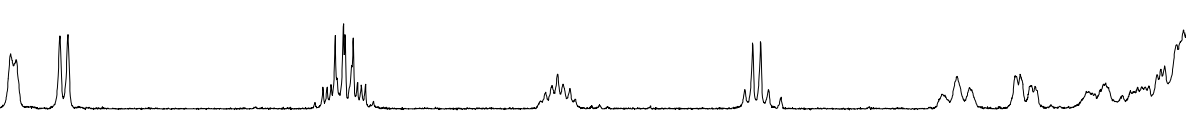




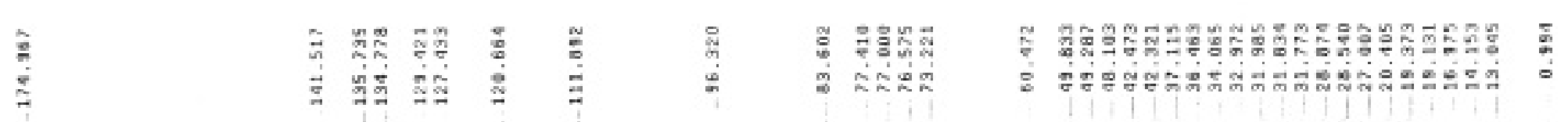

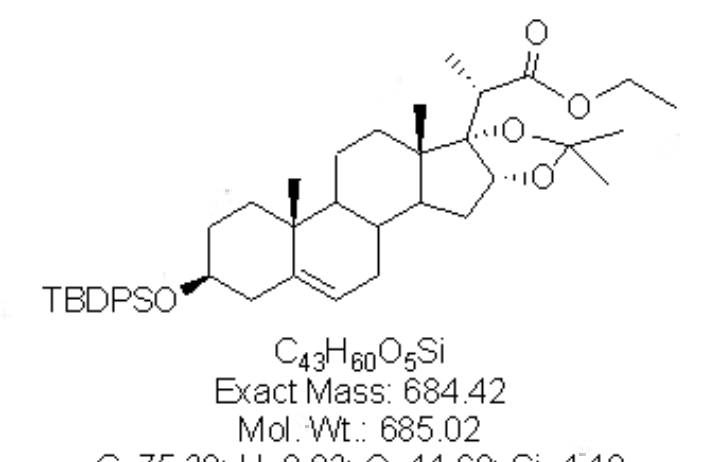

C. $75.39 ; \mathrm{H}, 8.83 ; 0,11.68 ; \mathrm{Si}, 4.10$

$\mathrm{CDCl}_{3}$

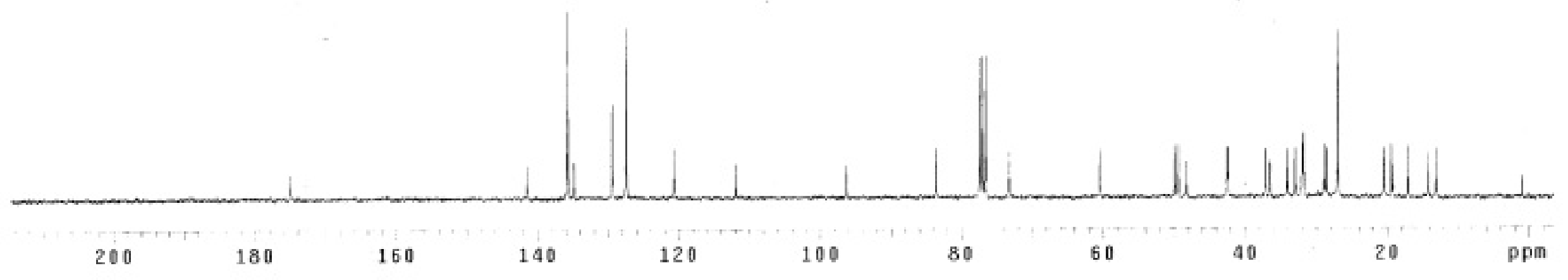

${ }^{13} \mathrm{CNMR}$ spectrum $(75 \mathrm{MHz})$ of 13 in $\mathrm{CDCl}_{3}$ 

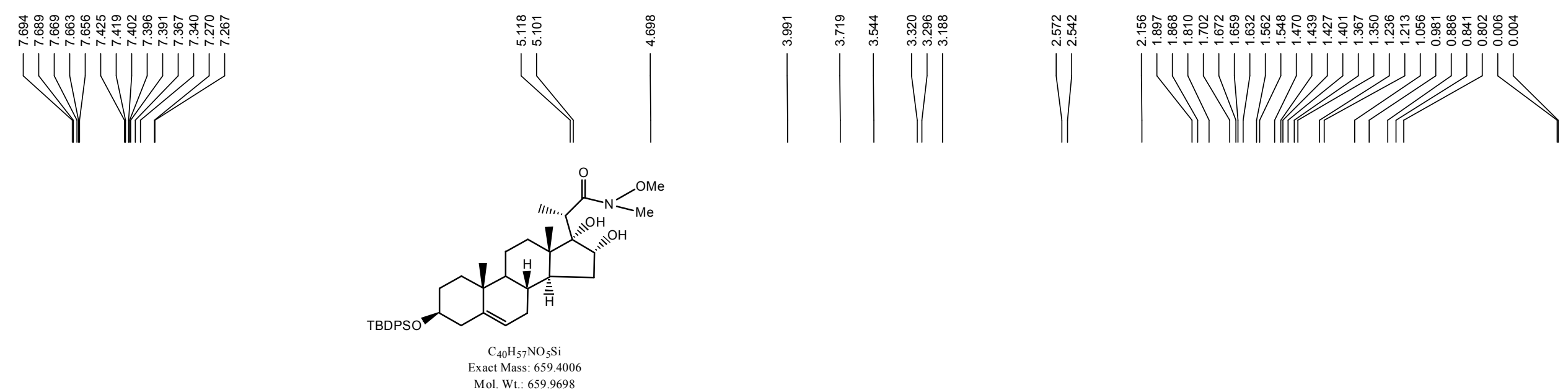

C, 72.80; H, 8.71; N, 2.12; O, $12.12 ; \mathrm{Si}, 4.26$

$\mathrm{CDCl}_{3}$
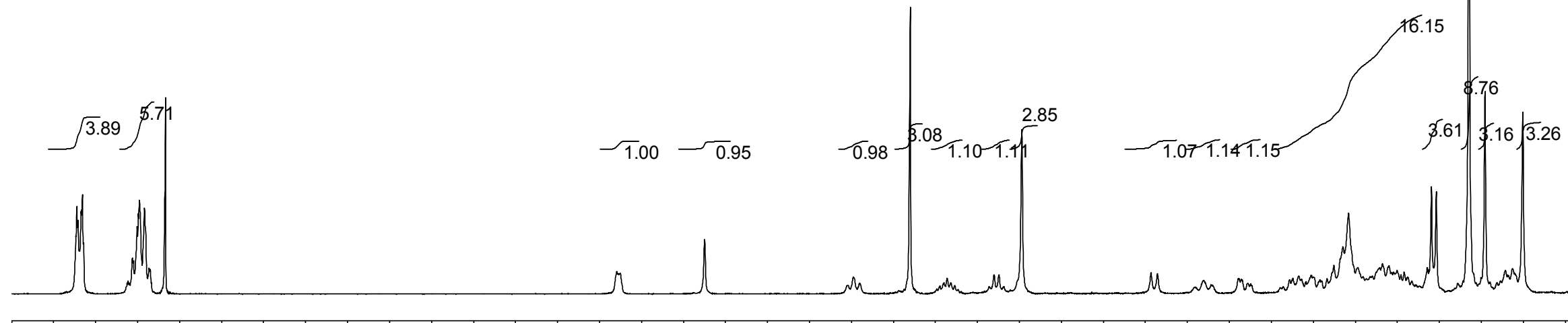

4

3

2

1 
c13 yubs $55-9$ in ede 13

Pulse Sequence: s2pul

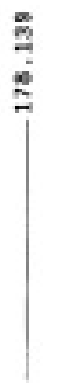

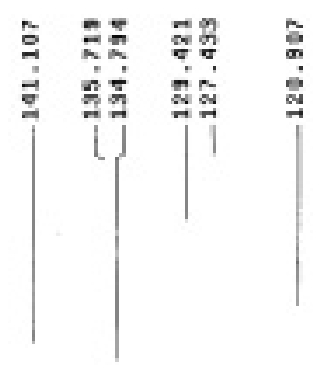

รับำ

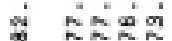

ijia

(i) $\tilde{\mathrm{t}}$

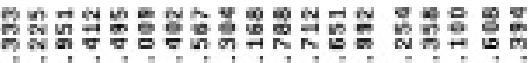

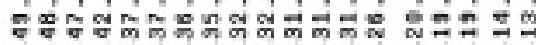

(L) (L(L)]
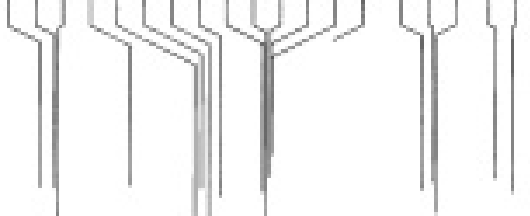

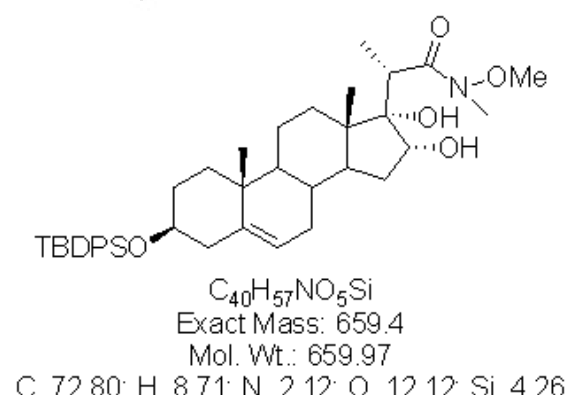

C. $72.80 ; \mathrm{H}, 8.71 ; \mathrm{N}, 2.12 ; \mathrm{O}, 12.12 ; \mathrm{Si}, 4.26$

$$
\mathrm{CDCl}_{3}
$$

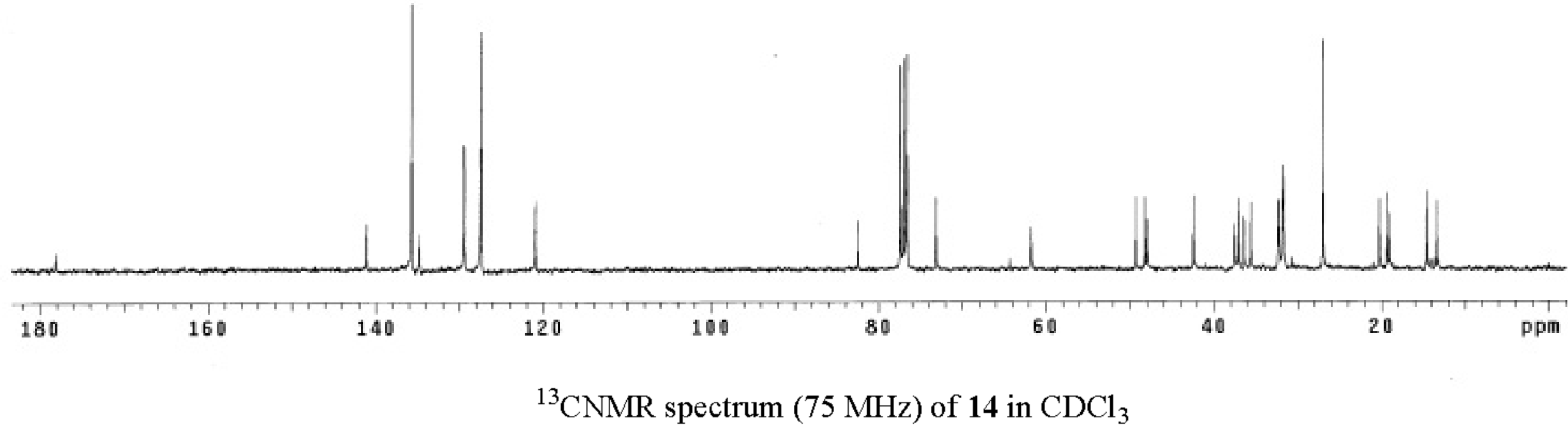




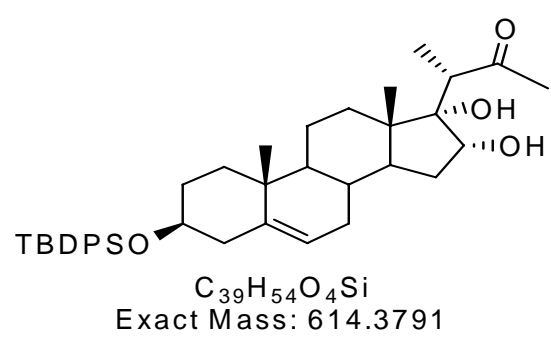

.

C, $76.17 ; \mathrm{H}, 8.85 ; \mathrm{O}, 10.41 ; \mathrm{Si}, 4.57$

$$
\mathrm{CDCl}_{3}
$$




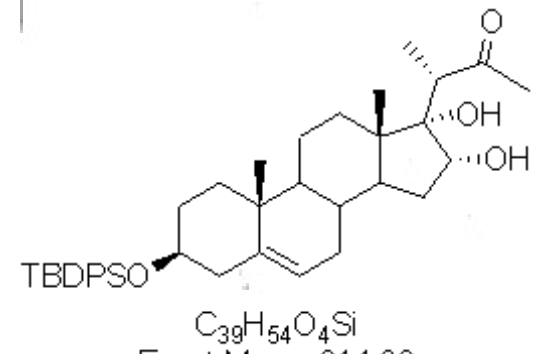

Eact Mass. 614.38

Mol Wt: 61493

C. $76.17 ; \mathrm{H}, 8.85 ; \mathrm{O}, 10.41 ; \mathrm{Si}, 4.57$ $\mathrm{CDCl}_{3}$

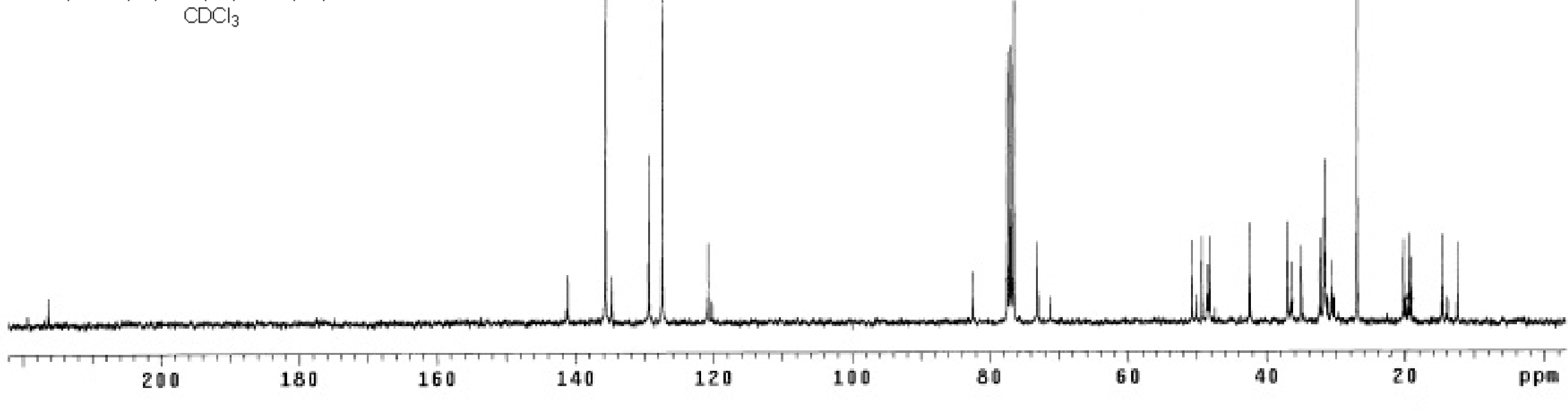

${ }^{1} \mathrm{H} \mathrm{NMR}$ spectrum $(300 \mathrm{MHz})$ of 15 in $\mathrm{CDCl}_{3}$ 


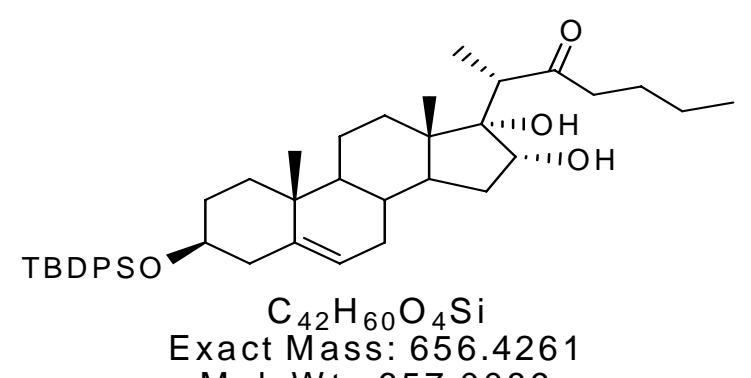

Mass: 656.4261

C, 76.78; H, 9.20; O, 9.74; Si, 4.27 $\mathrm{CDCl}_{3}$ 


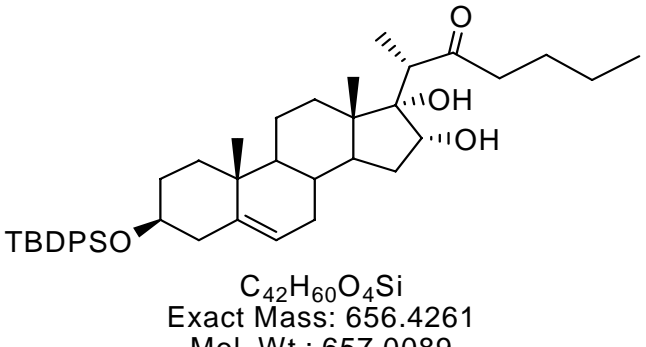

Mol $W \mathrm{Wt}: 057.0089$

C, 76.78; H, 9.20; O, 9.74; Si, 4.27

$$
\mathrm{CDCl}_{3}
$$




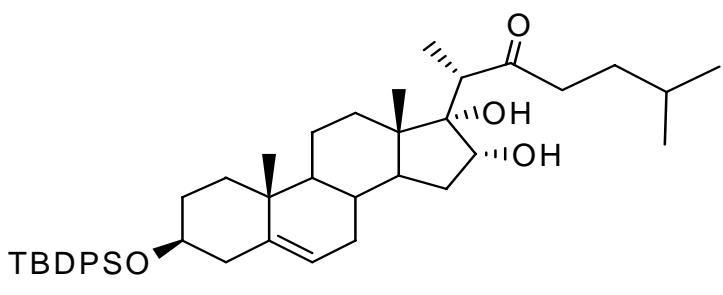

$\mathrm{C}_{43} \mathrm{H}_{62} \mathrm{O}_{4} \mathrm{Si}$

Exact Mass: 670.4417

Mol. Wt.: 671.0355

C, $76.96 ; \mathrm{H}, 9.31 ; \mathrm{O}, 9.54 ; \mathrm{Si}, 4.19$

$\mathrm{CDCl}_{3}$ 


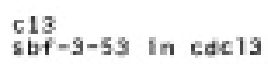

Pulse sequences: s2pul



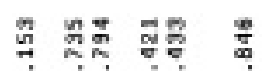

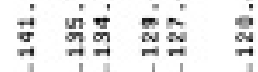
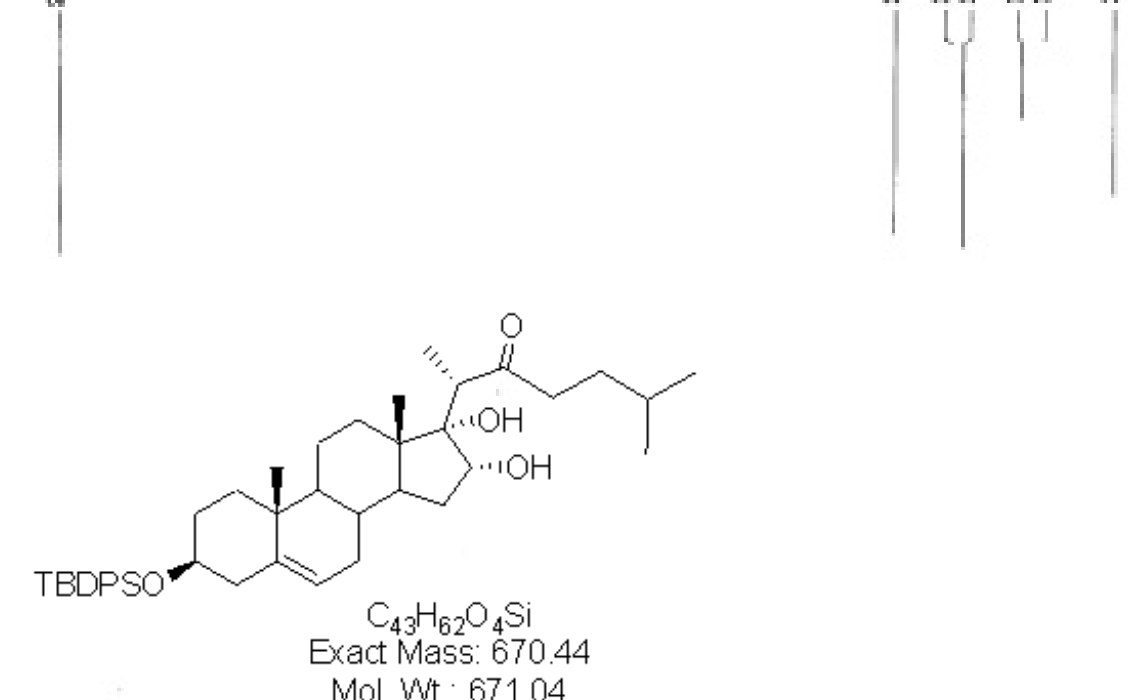

C. $76.96 ; H, 9.31 ; 0,9.54 ;$ Si, 4.19

$\mathrm{CDCl}_{3}$

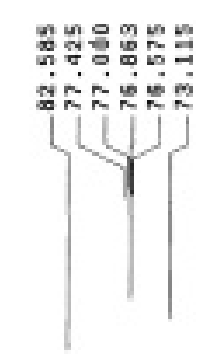

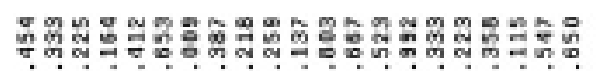

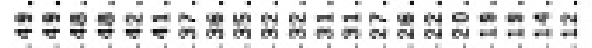

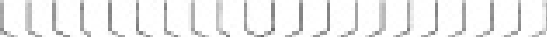

7
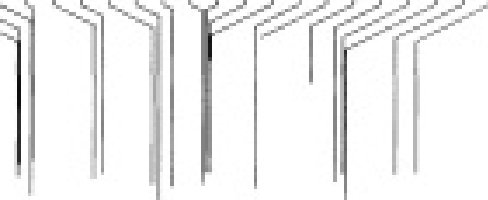

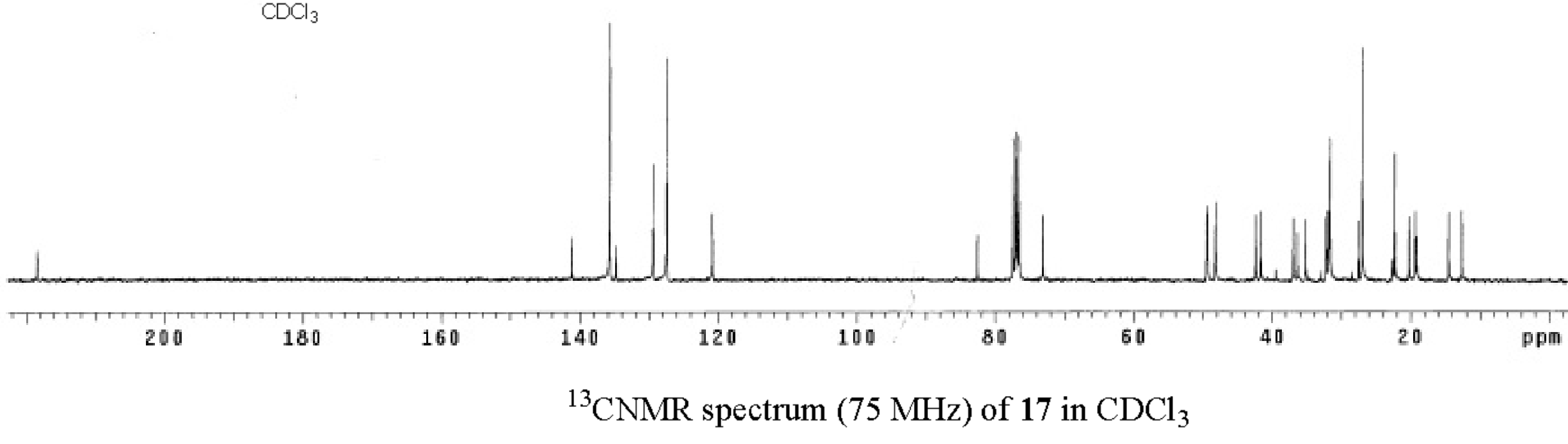




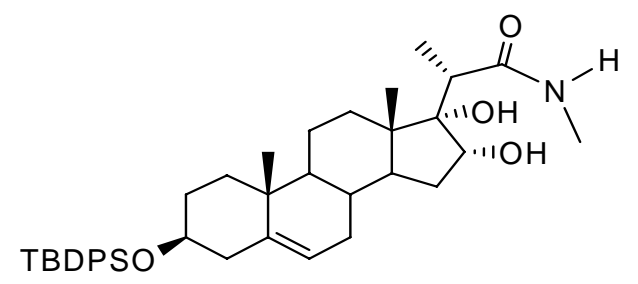

$\mathrm{C}_{39} \mathrm{H}_{55} \mathrm{NO}_{4} \mathrm{Si}$

Exact Mass: 629.39

Mol. Wt: 629.9438

C, $74.36 ; \mathrm{H}, 8.80 ; \mathrm{N}, 2.22 ; \mathrm{O}, 10.16 ; \mathrm{Si}, 4.46$

$\mathrm{CDCl}_{3}$

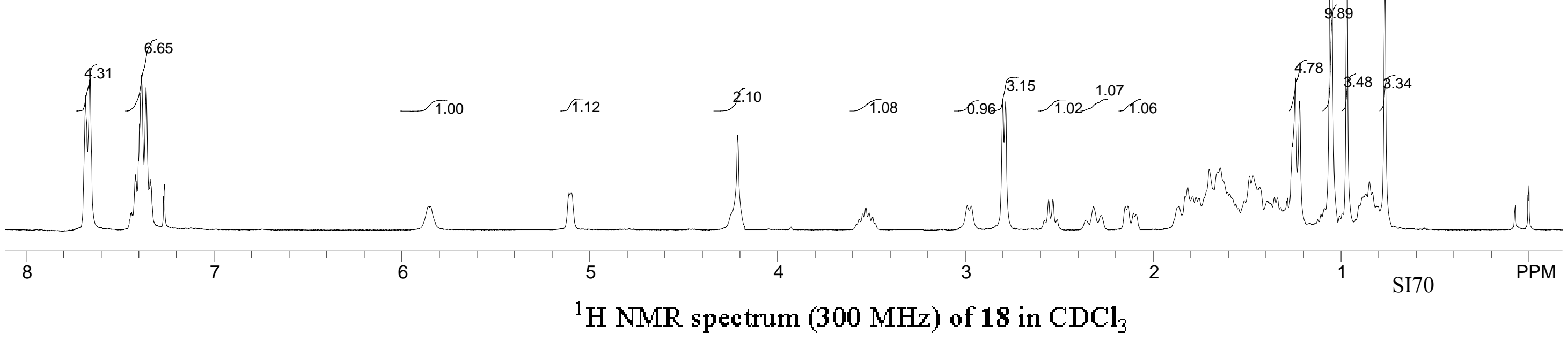




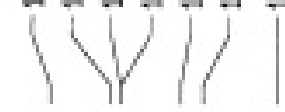

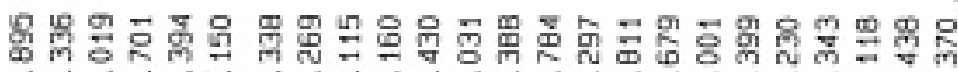

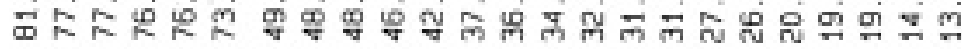

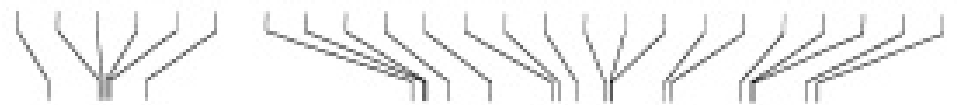

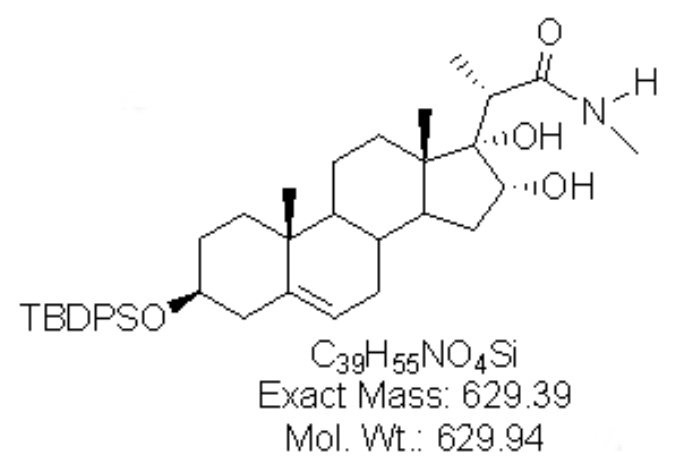

C. $74.36 ; H, 8.80 ; N, 2.22 ; 0,10.16 ; \mathrm{Si}, 4.46$ $\mathrm{CDCl}_{3}$
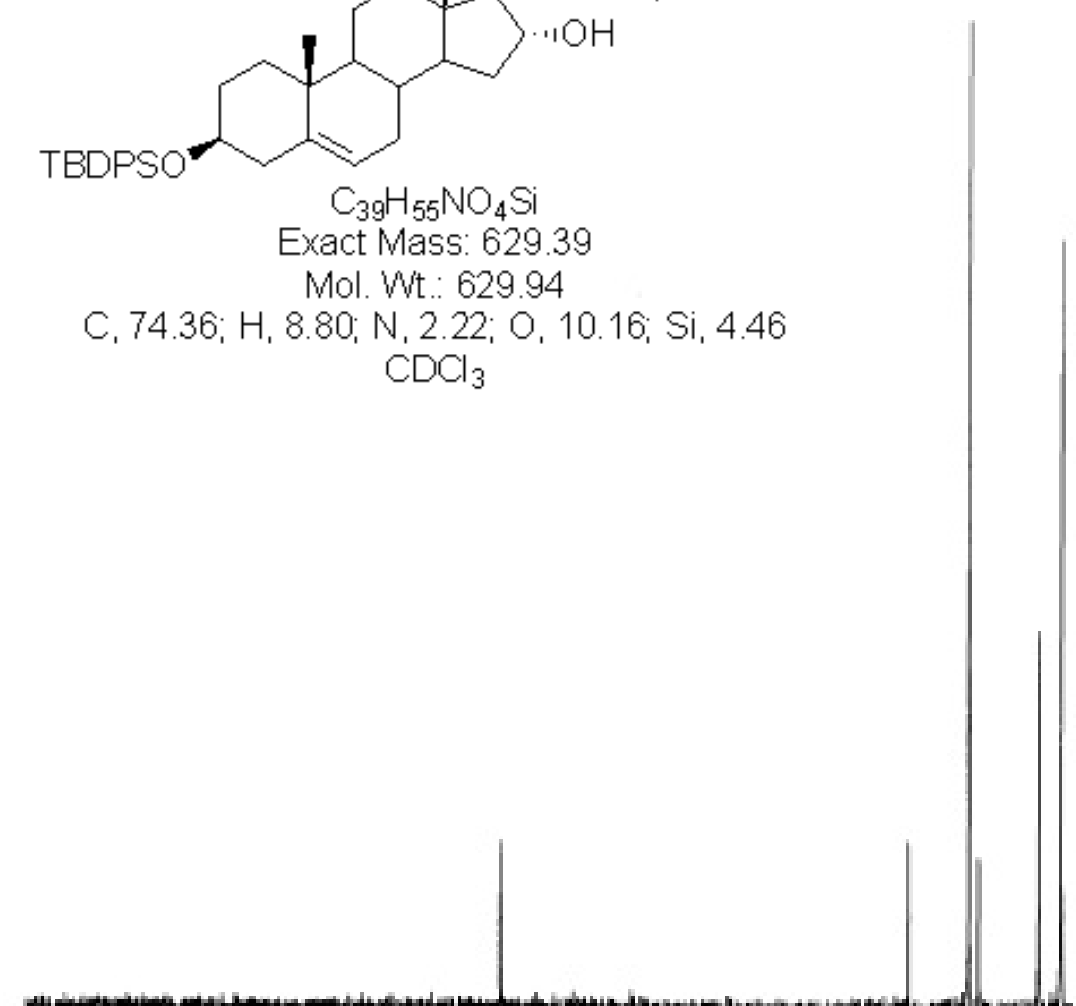

${ }^{13} \mathrm{CNMR}$ spectrum $(100 \mathrm{MHz})$ of 18 in $\mathrm{CDCl}_{3}$ 


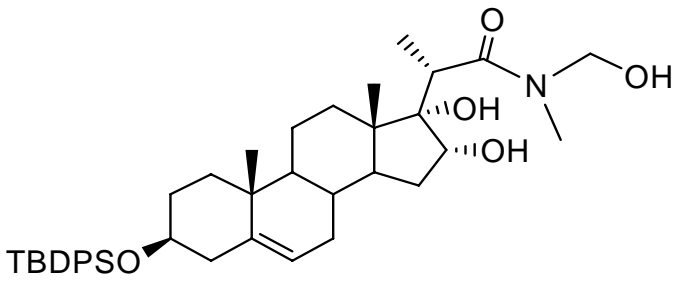

$\mathrm{C}_{40} \mathrm{H}_{57} \mathrm{NO}_{5} \mathrm{Si}$

Exact Mass: 659.4006

Mol. Wt: 659.9698

C, $72.80 ; \mathrm{H}, 8.71 ; \mathrm{N}, 2.12 ; \mathrm{O}, 12.12 ; \mathrm{Si}, 4.26$

$\mathrm{CDCl}_{3}$

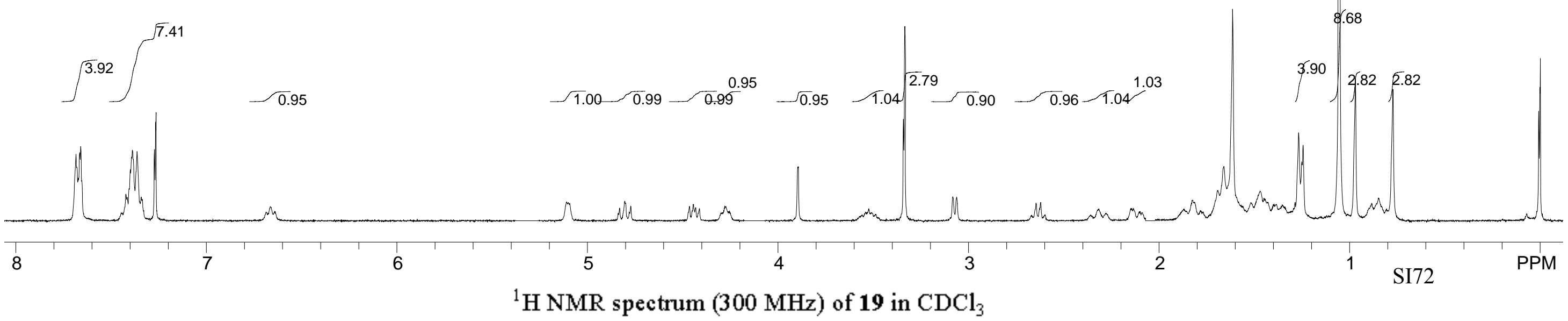




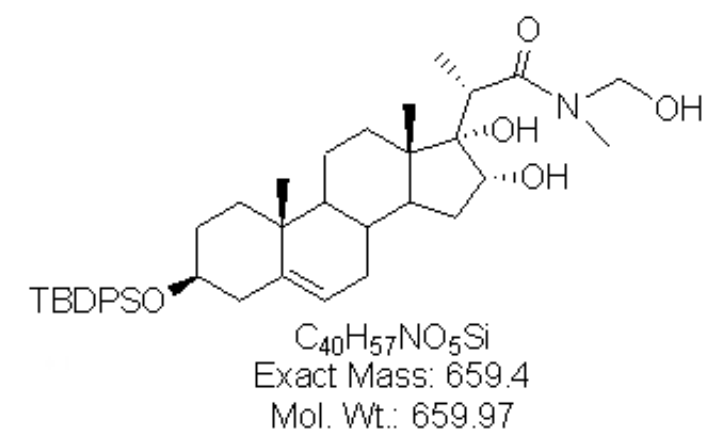

C. $72.80 ; \mathrm{H}, 8.71 ; \mathrm{N}, 2.12 ; 0,12.12 ; \mathrm{Si}, 4.26$ $\mathrm{CDCl}_{3}$
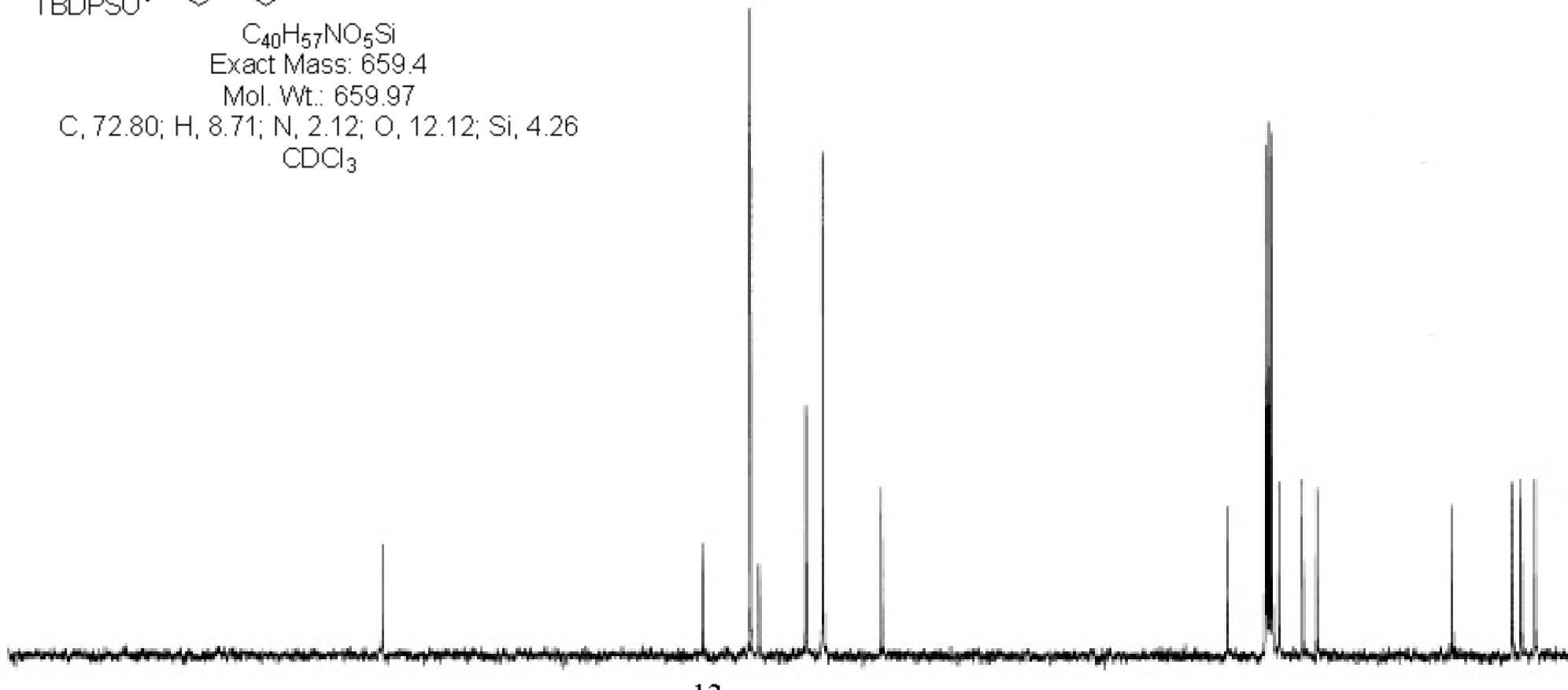

${ }^{13} \mathrm{CNMR}$ spectrum $(75 \mathrm{MHz})$ of 19 in $\mathrm{CDCl}_{3}$ 


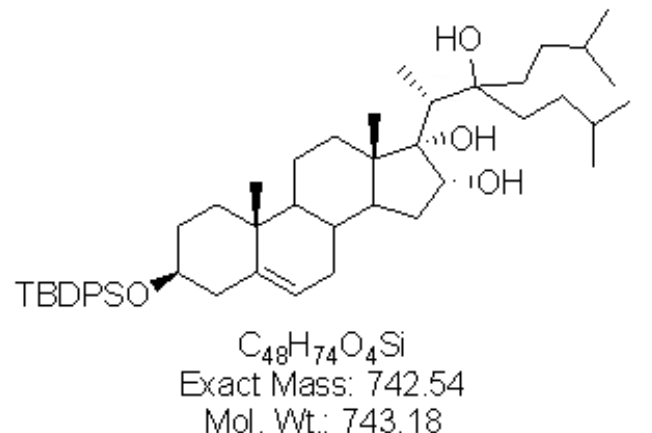

C. $77.57 ; \mathrm{H}, 10.04 ; 0,8.61 ; \mathrm{Si}, 3.78$ $\mathrm{CDCl}_{3}$

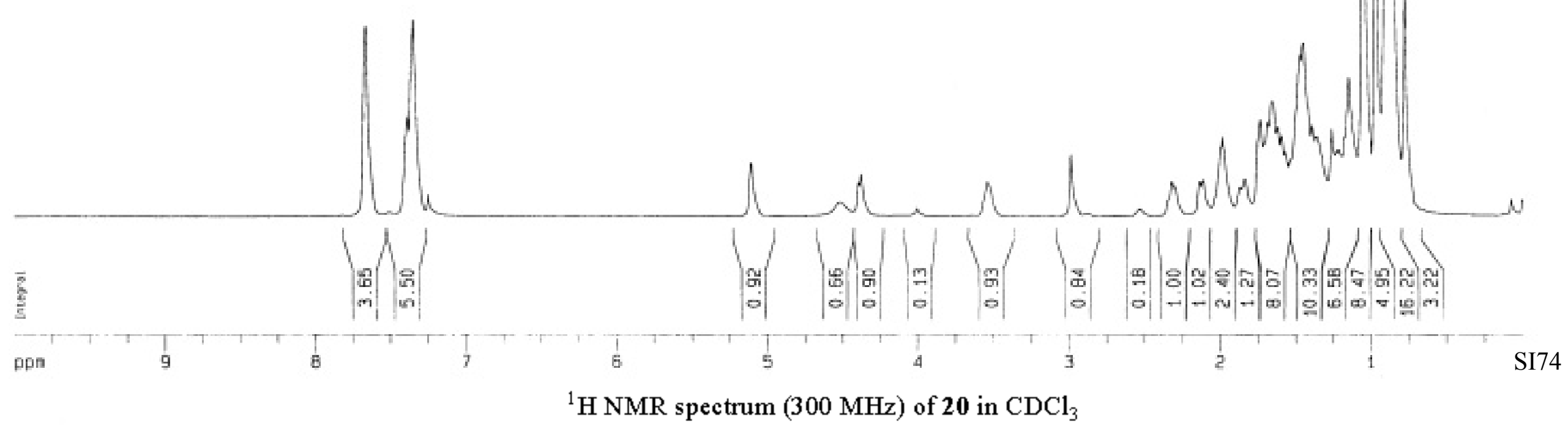




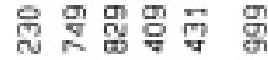

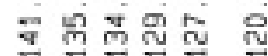
$1 / 11$

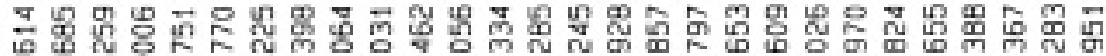

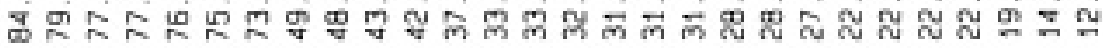
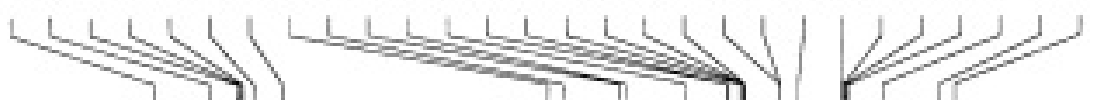

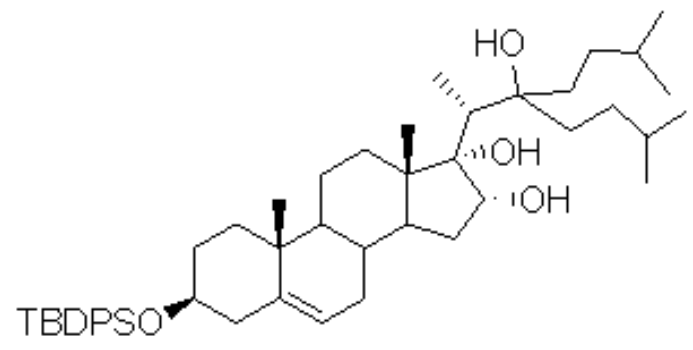

$\mathrm{C}_{48} \mathrm{H}_{74} \mathrm{O}_{4} \mathrm{Si}$

Exact Mass: 742.54

Mol Wh: 74318

C. $77.57 ; \mathrm{H}, 10.04 ; 0.8 .61 ; \mathrm{Si}, 3.78$

$\mathrm{CDCl}_{3}$

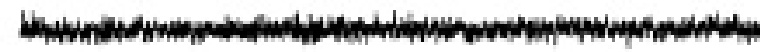



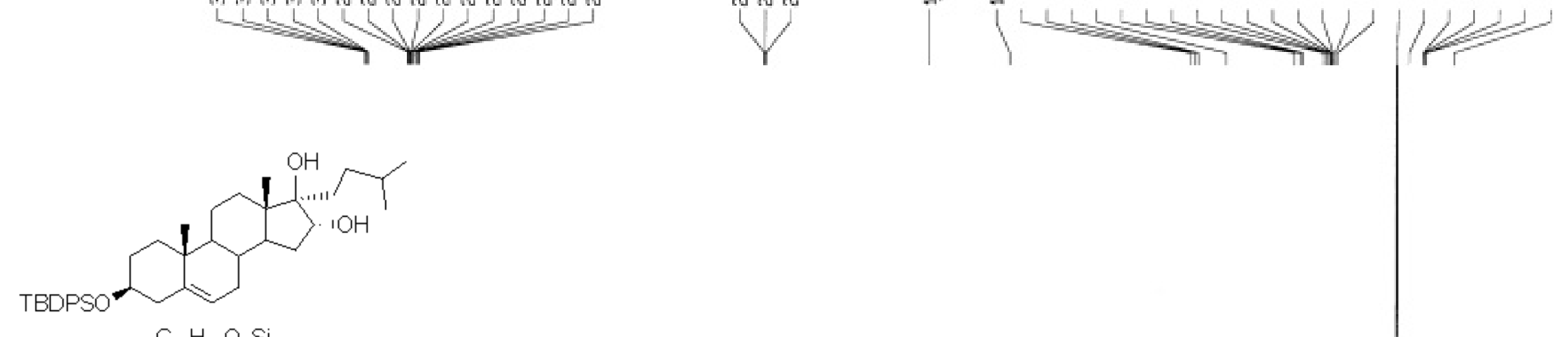

$\mathrm{C}_{40} \mathrm{H}_{58} \mathrm{O}_{3} \mathrm{Si}$

Exact Mass: 614.42

Mol. Wt: 614.97

C. $78.12 ; \mathrm{H}, 9.51 ; 0,7.80 ; \mathrm{Si}, 4.57$

$\mathrm{CDCl}_{3}$
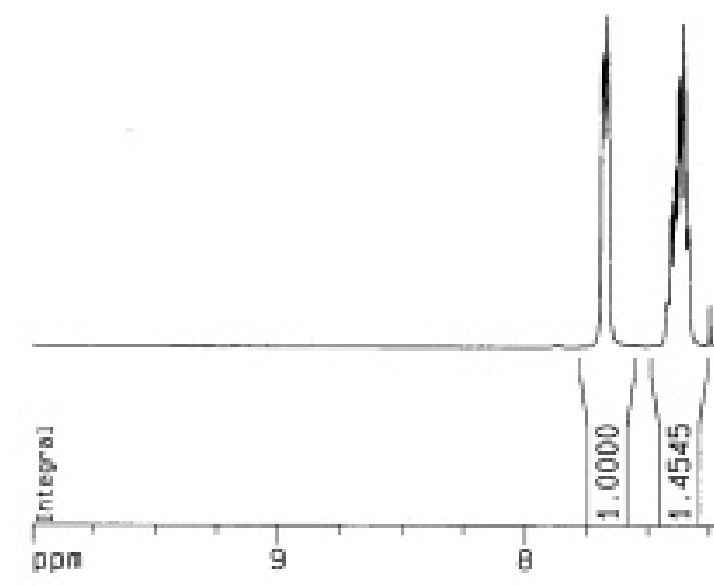

${ }^{1} \mathrm{H}$ NMR spectrum $(400 \mathrm{MHz})$ of 22 in $\mathrm{CDCl}_{3}$ 

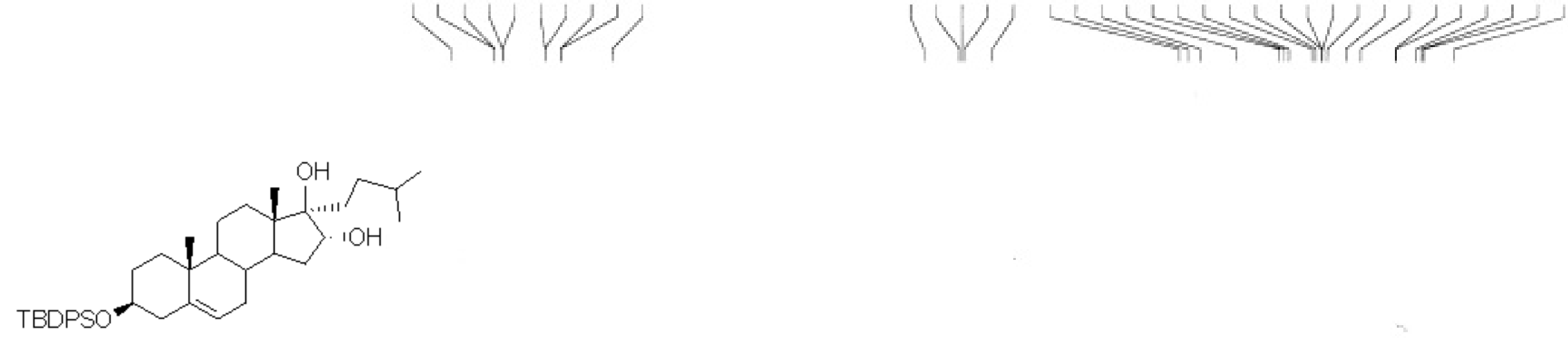

$\mathrm{C}_{40} \mathrm{H}_{58} \mathrm{O}_{3} \mathrm{Si}$

Exact Mass: 614.42

Mol. Wt: 614.97

C. $78.12 ; \mathrm{H}, 9.51 ; 0.7 .80 ; \mathrm{Si}, 4.57$

$\mathrm{CDCl}_{3}$

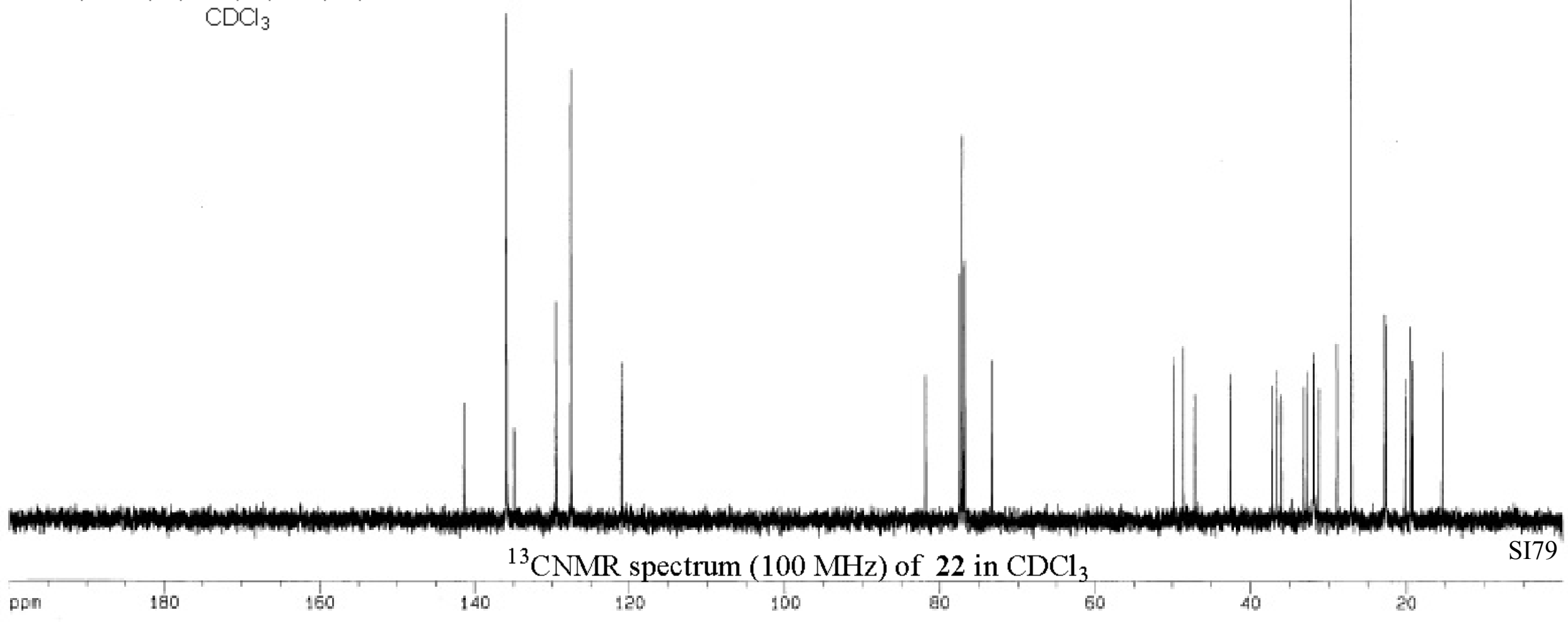




\section{$\mathrm{C}_{38} \mathrm{H}_{52} \mathrm{O}_{4} \mathrm{Si}$}

TBDPSO

Exact Mass: 600.36

Mol. W t.: 600.9
C, $75.95 ; \mathrm{H}, 8.72 ; \mathrm{O}, 10.65 ; \mathrm{Si}, 4.67$ 


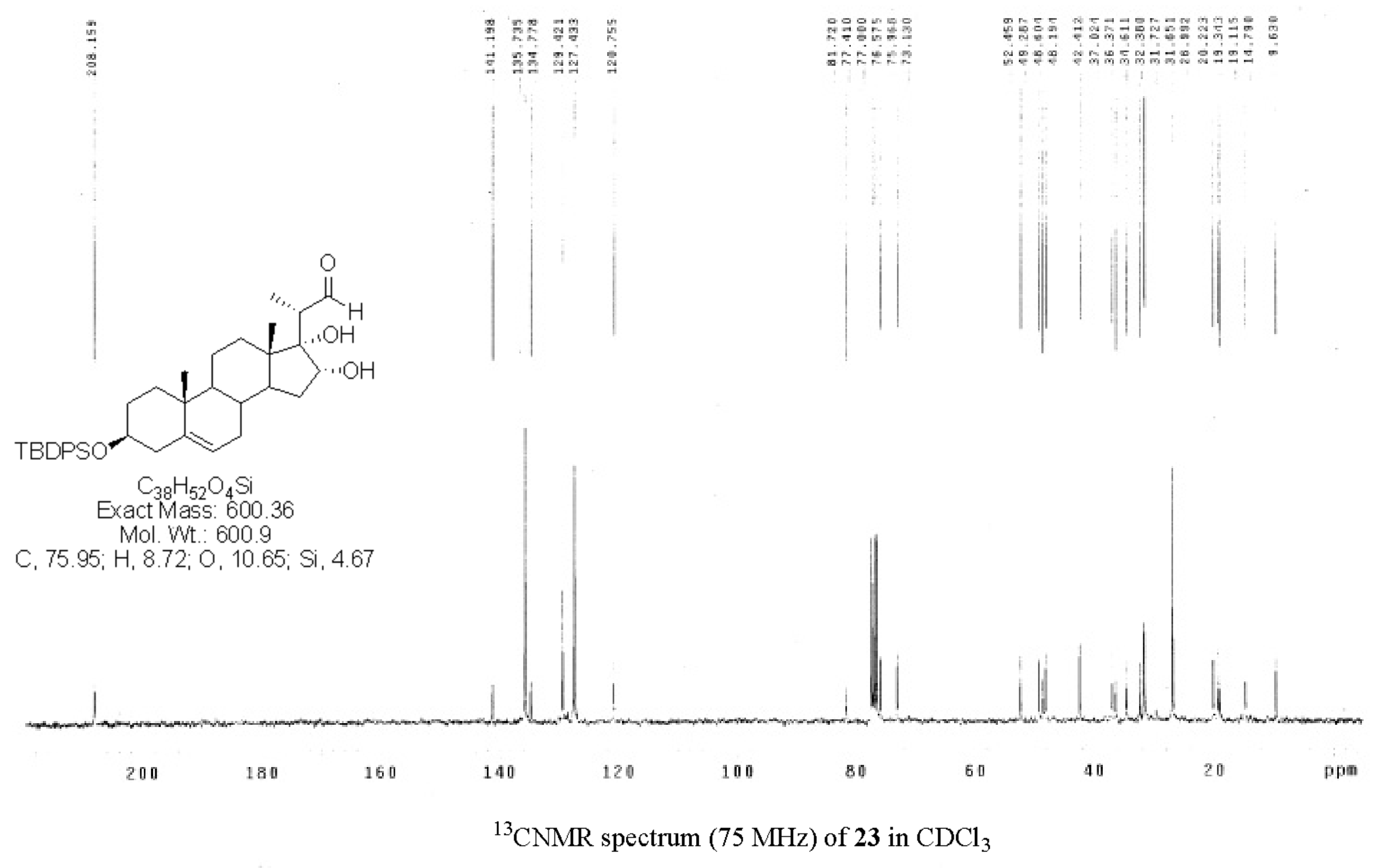




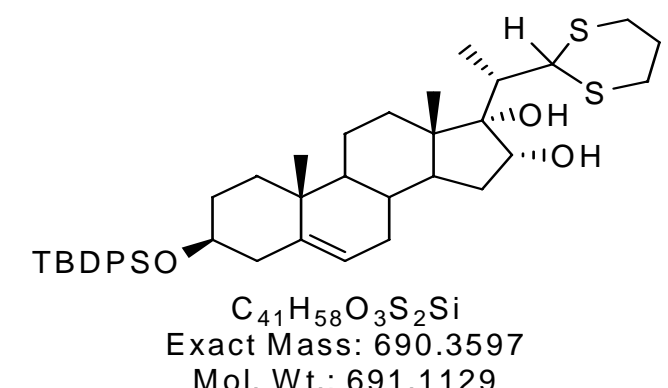

C, $71.25 ; \mathrm{H}, 8.46 ; \mathrm{O}, 6.95 ; \mathrm{S}, 9.28 ; \mathrm{Si}, 4.06$ $\mathrm{CDCl}_{3}$
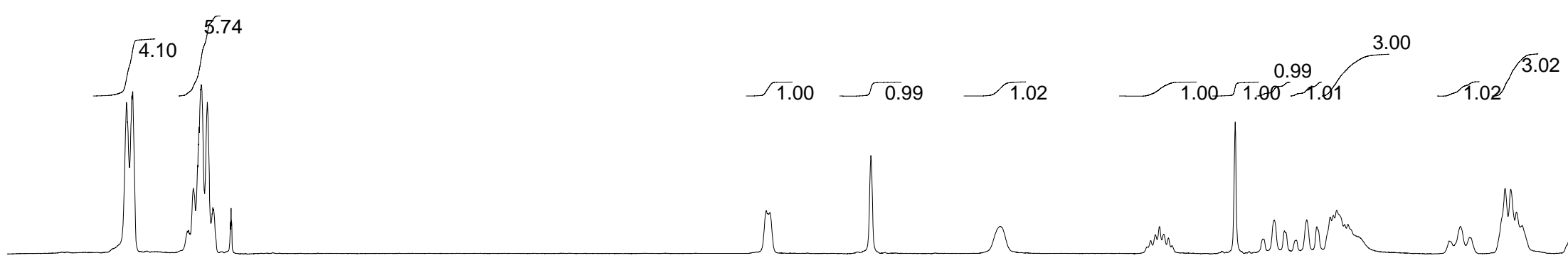

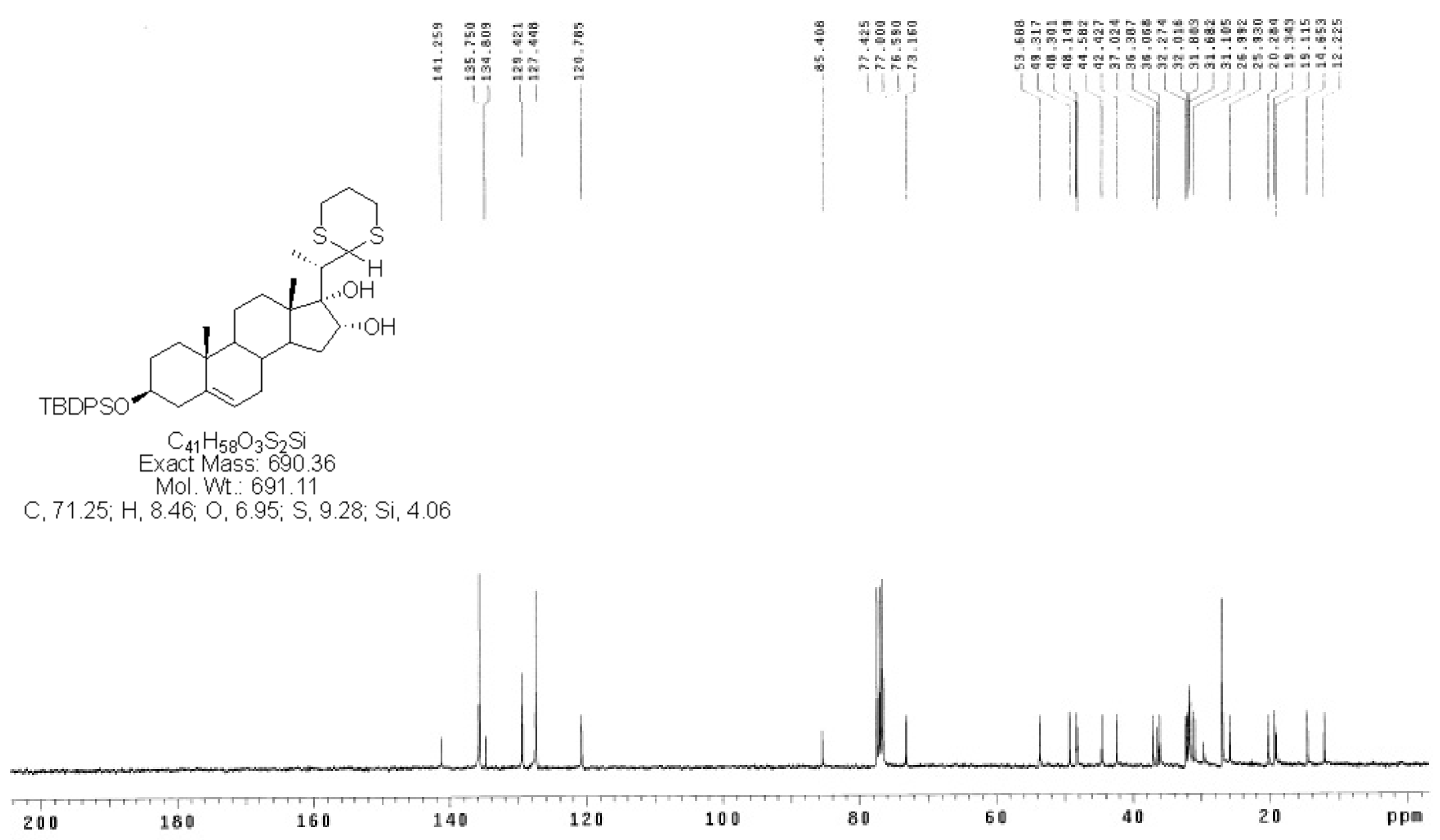

${ }^{13} \mathrm{CNMR}$ spectrum $\left(75 \mathrm{MHz}\right.$ ) of 24 in $\mathrm{CDCl}_{3}$ 


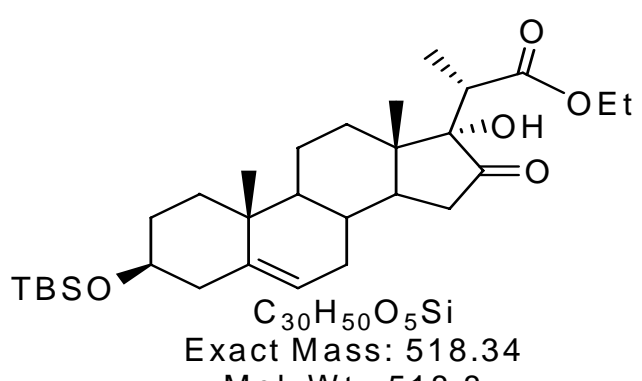

Mol. Wt: 51

C, $69.45 ; \mathrm{H}, 9.71 ; \mathrm{O}, 15.42 ; \mathrm{Si}, 5.41$

$\mathrm{CDCl}_{3}$

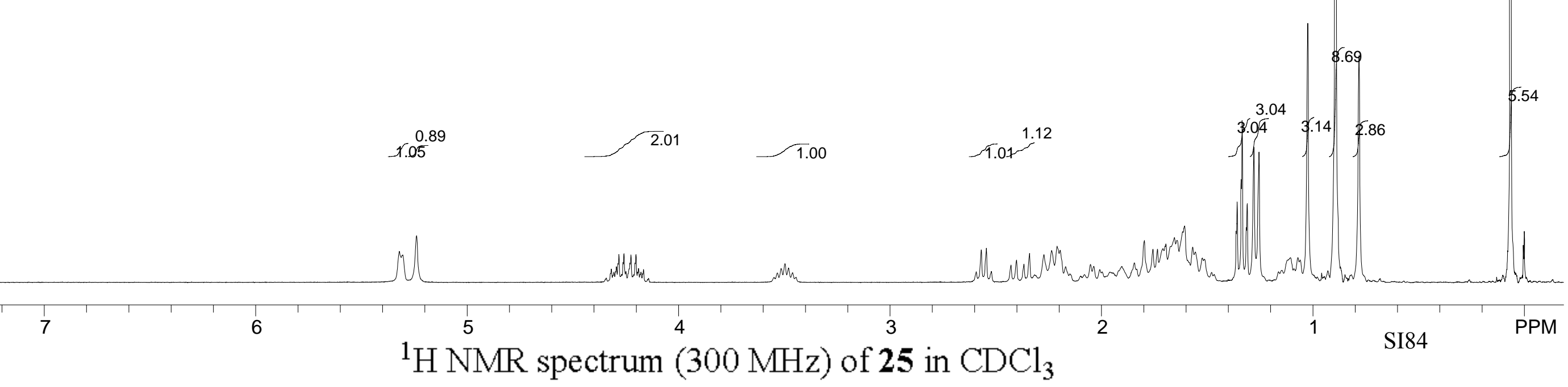




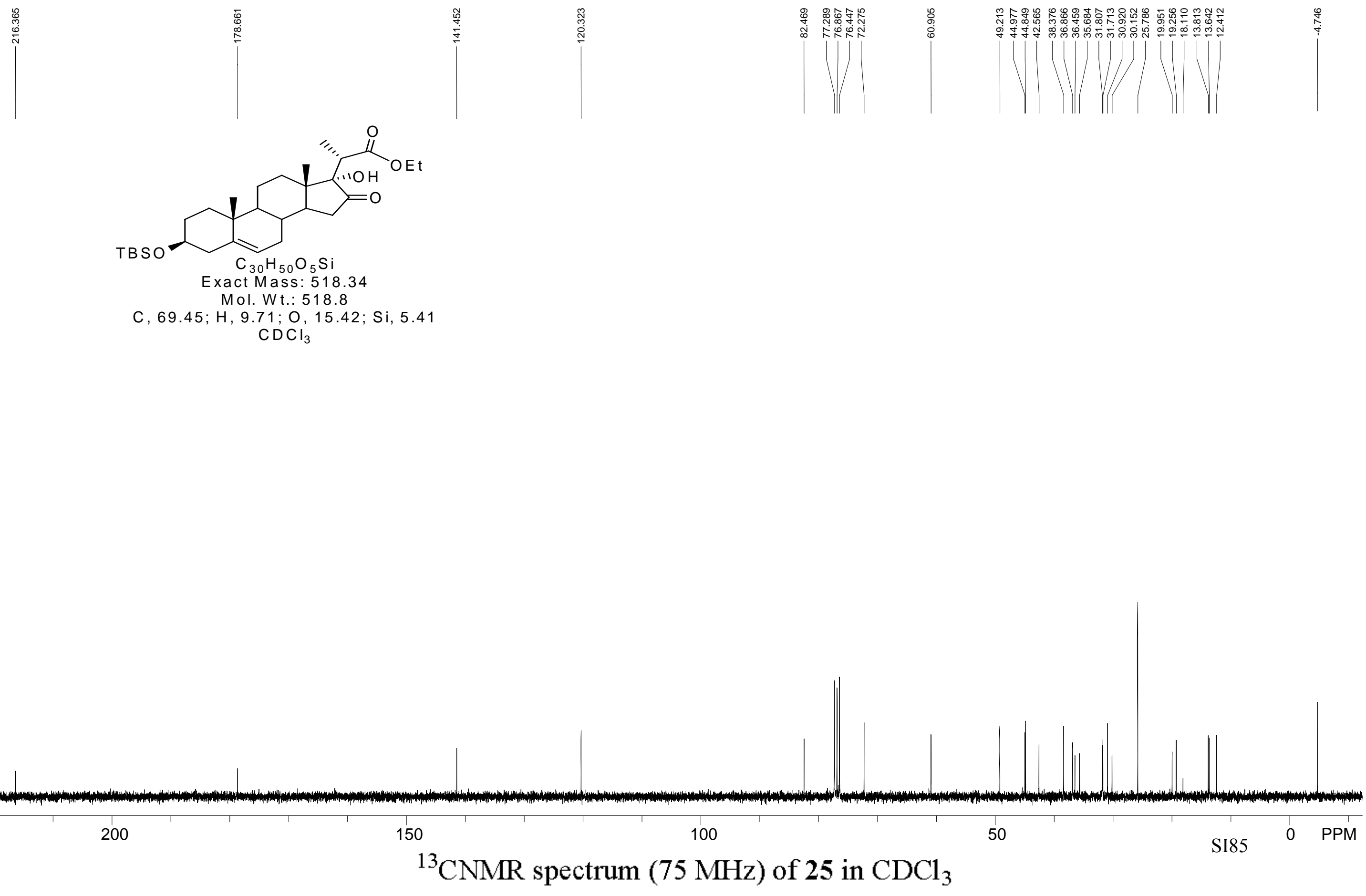




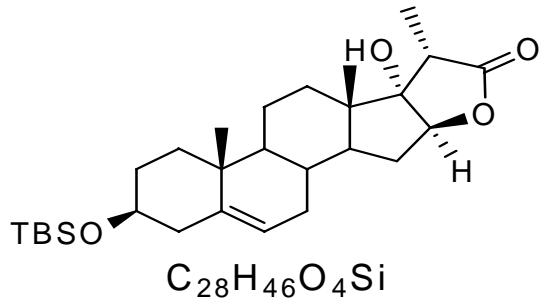

Exact Mass: 474.3165

Mol. Wt.: 474.7479

C, $70.84 ; \mathrm{H}, 9.77 ; \mathrm{O}, 13.48 ; \mathrm{Si}, 5.92$

$\mathrm{CDCl}_{3}$

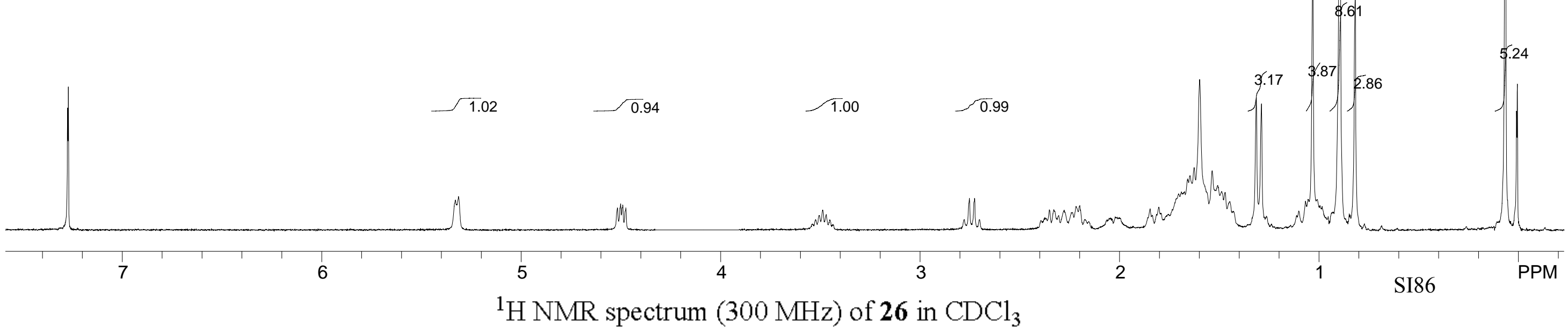




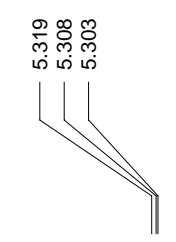

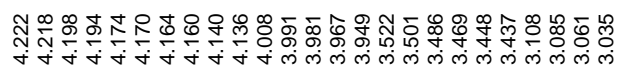

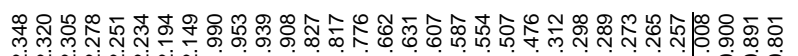

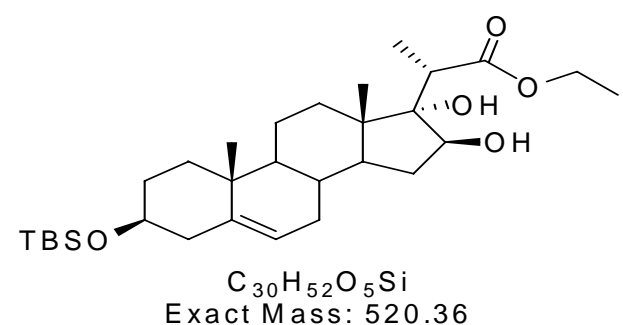

Mol. Wt.: 520.82

C, $69.18 ; \mathrm{H}, 10.06 ; 0,15.36 ; \mathrm{Si}, 5.39$

$\mathrm{CDCl}_{3}$
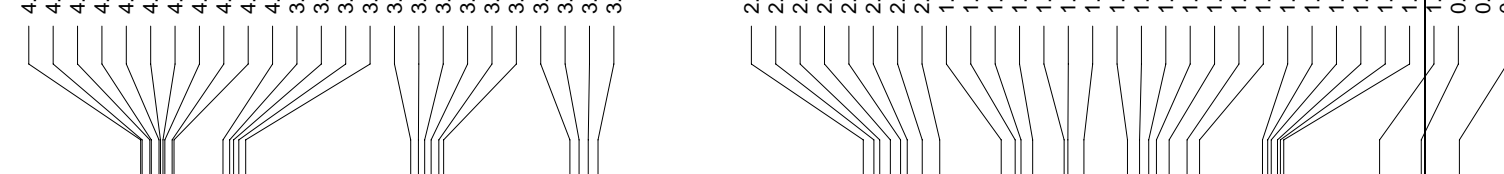
$\cong$

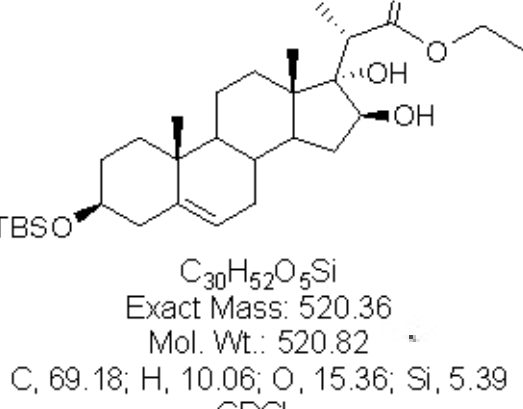

C. $69.18 ; H_{1}, 10.06 ; 0,15.36 ;$ Si, 5.39

$\mathrm{CDCl}_{3}$

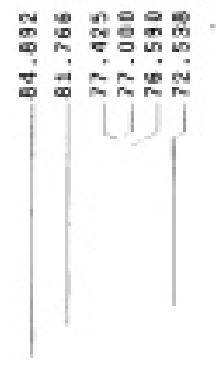

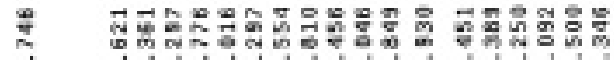

웅
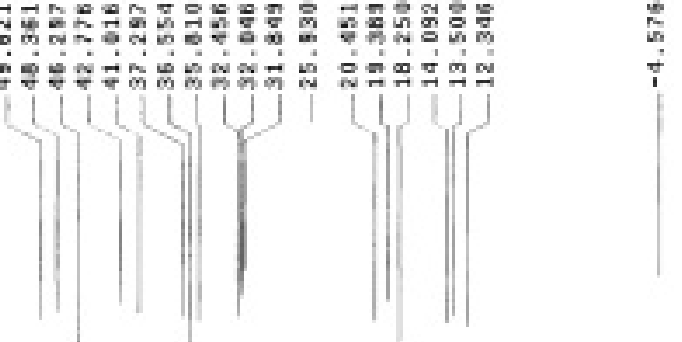

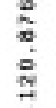

7
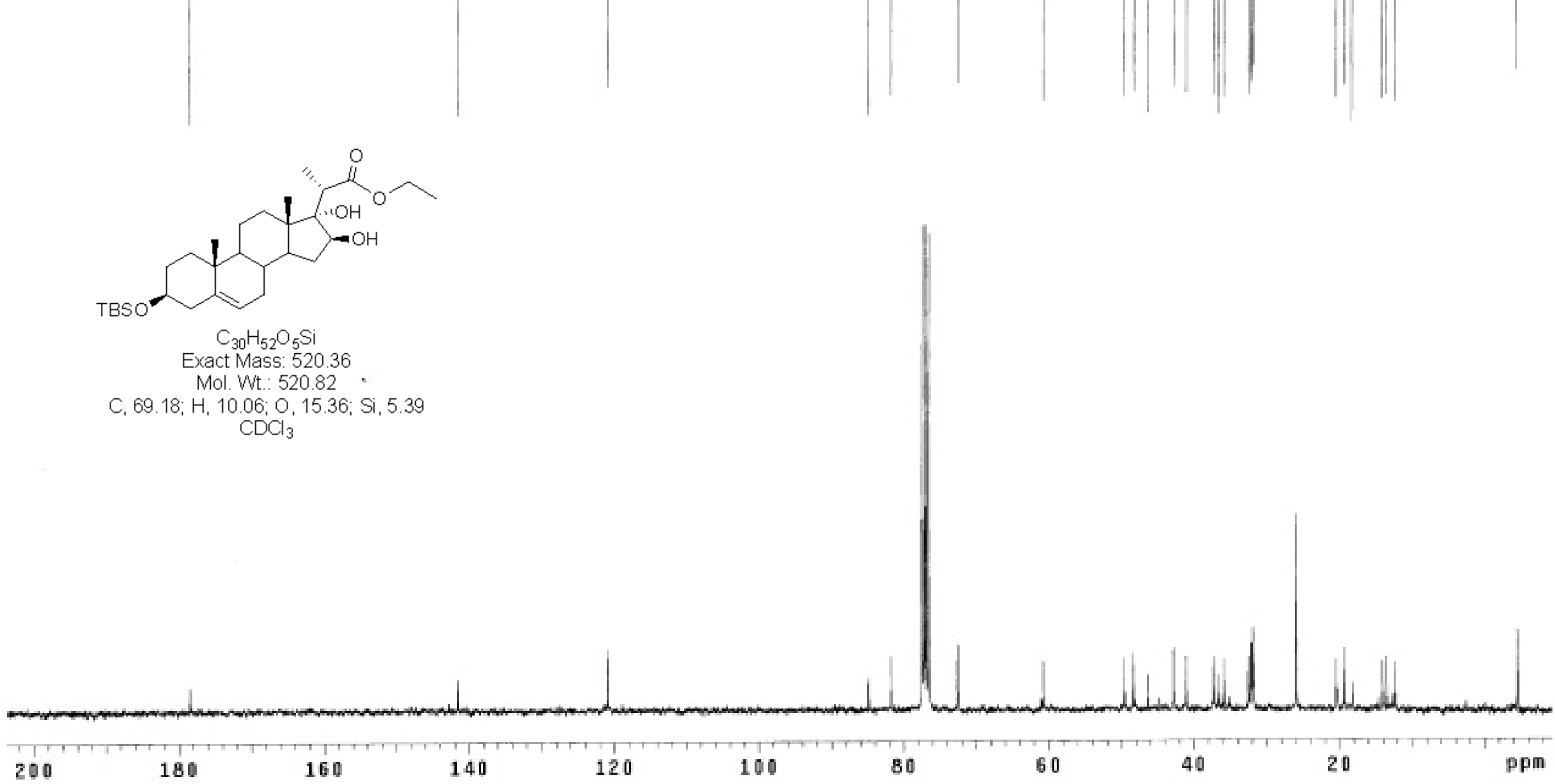

160

140

120

100

80

60

40

20

ppm

${ }^{1} \mathrm{H}$ NMR spectrum $(300 \mathrm{MHz})$ of $\mathbf{2 7}$ in $\mathrm{CDCl}_{3}$ 


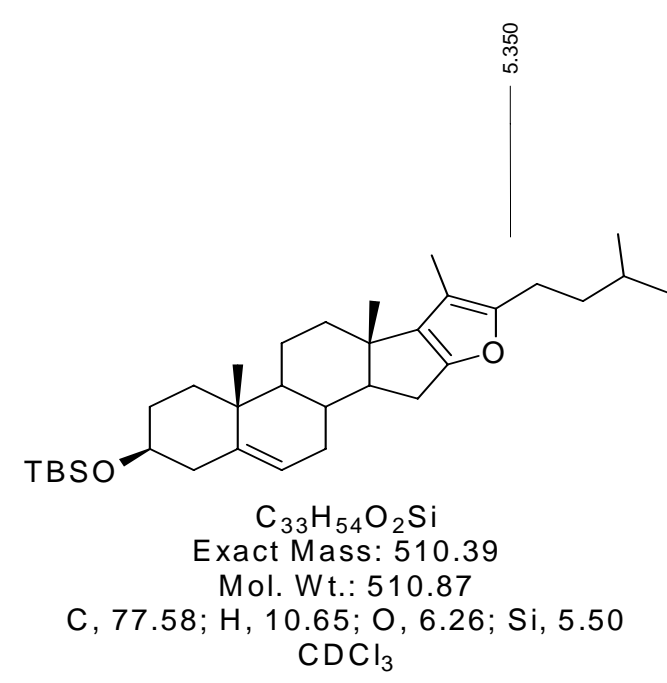

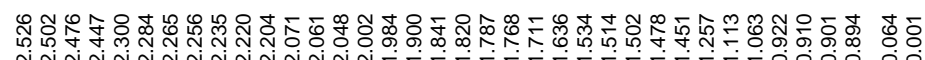

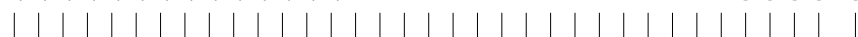




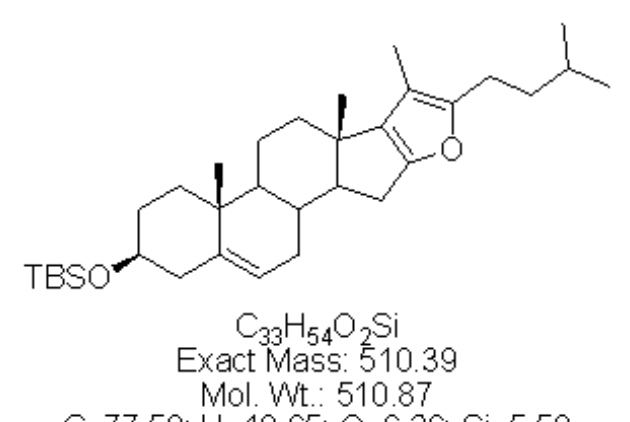

C. $77.58 ; \mathrm{H}, 10.65 ; 0,6.26 ;$ Si, 5.50

\section{(n)}

$\frac{1}{160}$ 


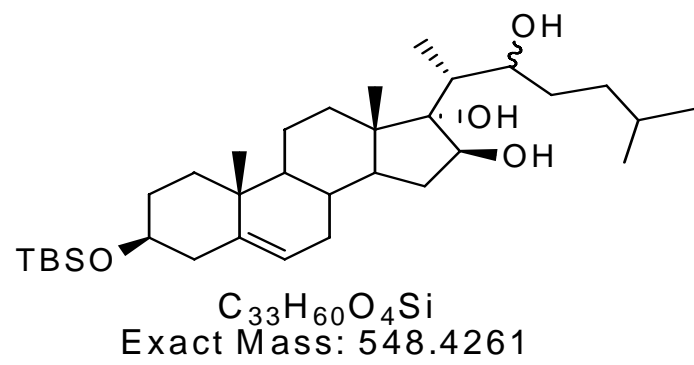

Exact Mass: 548

C, $72.21 ; \mathrm{H}, 11.02 ; \mathrm{O}, 11.66 ; \mathrm{Si}, 5.12$

$\mathrm{CDCl}_{3}$ 


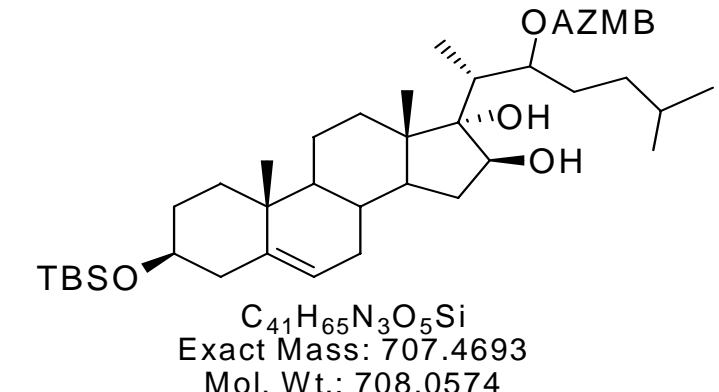

C, 69.55; H, 9.25: N, 5.93; O 11.30; Si, 3.97

$$
\mathrm{CDCl}_{3}
$$
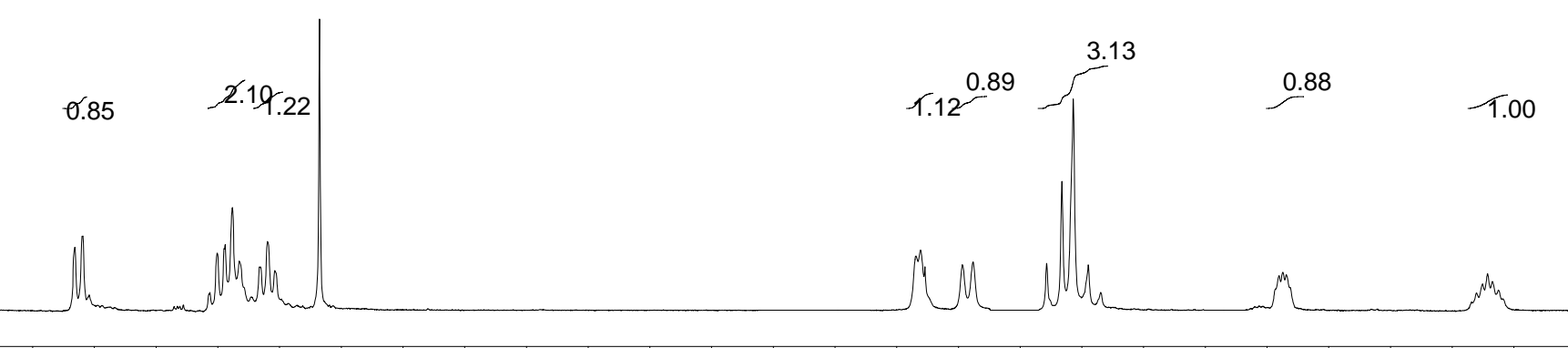

.00

1.10 


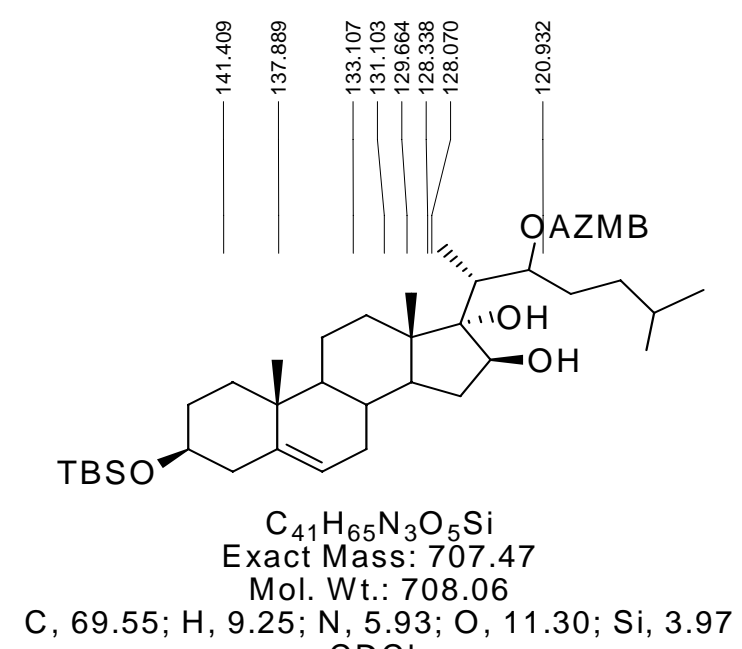

C, $69.55 ; \mathrm{H}, 9.25 ; \mathrm{N}, 5.93 ; \mathrm{O}, 11.30 ; \mathrm{Si}, 3.97$

$$
\mathrm{CDCl}_{3}
$$




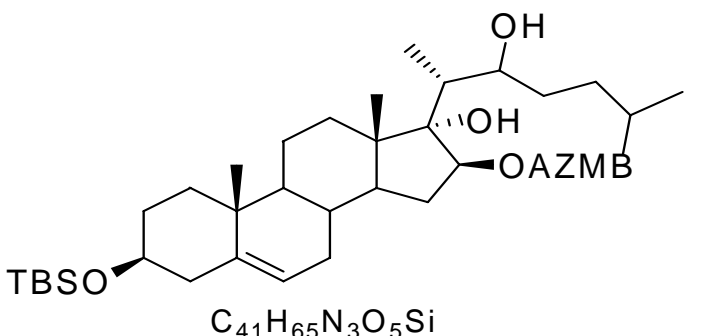

Exact Mass: 707.4693

Mol. Wt.: 708.0574

C, $69.55 ; \mathrm{H}, 9.25 ; \mathrm{N}, 5.93 ; \mathrm{O}, 11.30 ; \mathrm{Si}, 3.97$

$$
\mathrm{CDCl}_{3}
$$

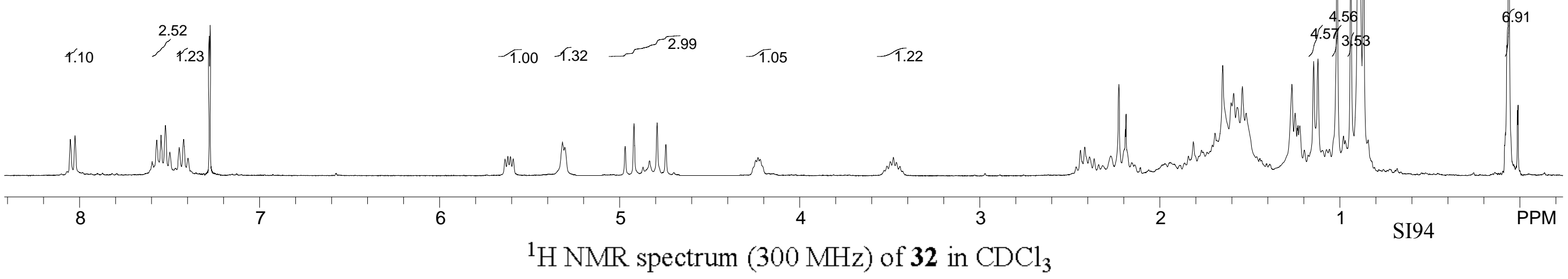




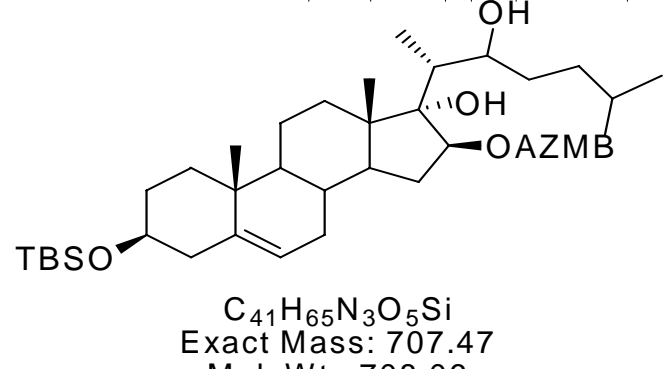

Mol. Wt: 708.06

C, $69.55 ; \mathrm{H}, 9.25 ; \mathrm{N}, 5.93 ; \mathrm{O}, 11.30 ; \mathrm{Si}, 3.97$

$$
\mathrm{CDCl}_{3}
$$

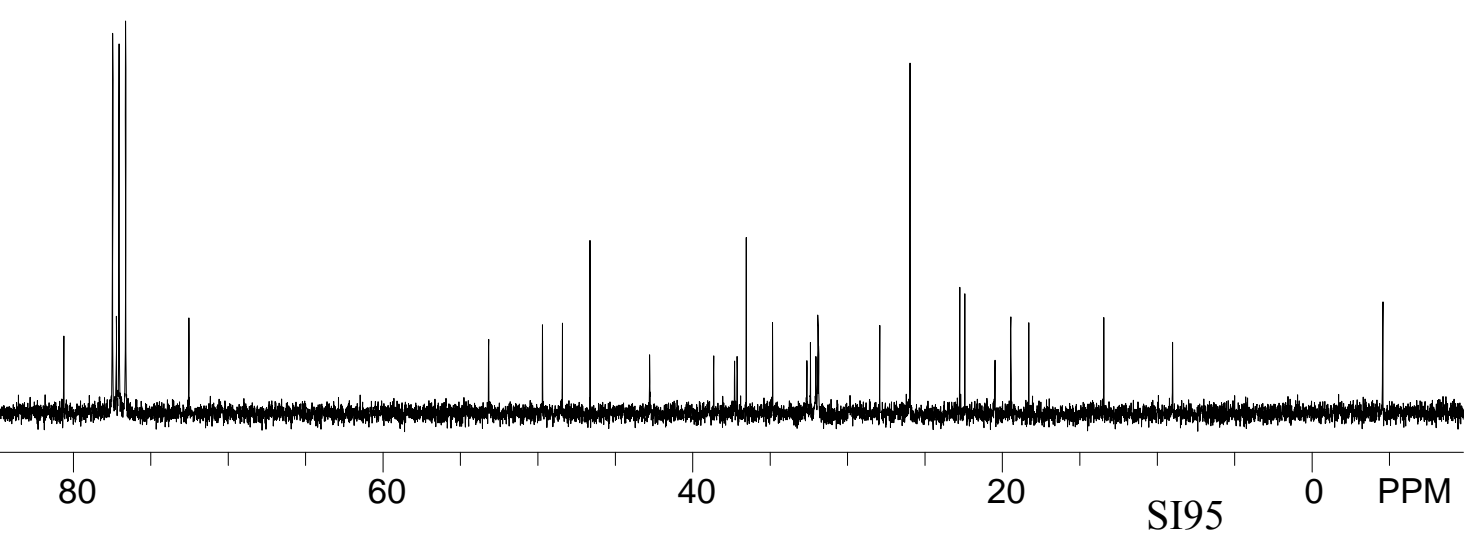

${ }^{13} \mathrm{CNMR}$ spectrum $(75 \mathrm{MHz})$ of 32 in $\mathrm{CDCl}_{3}$ 


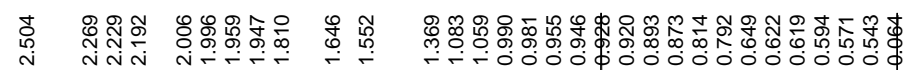

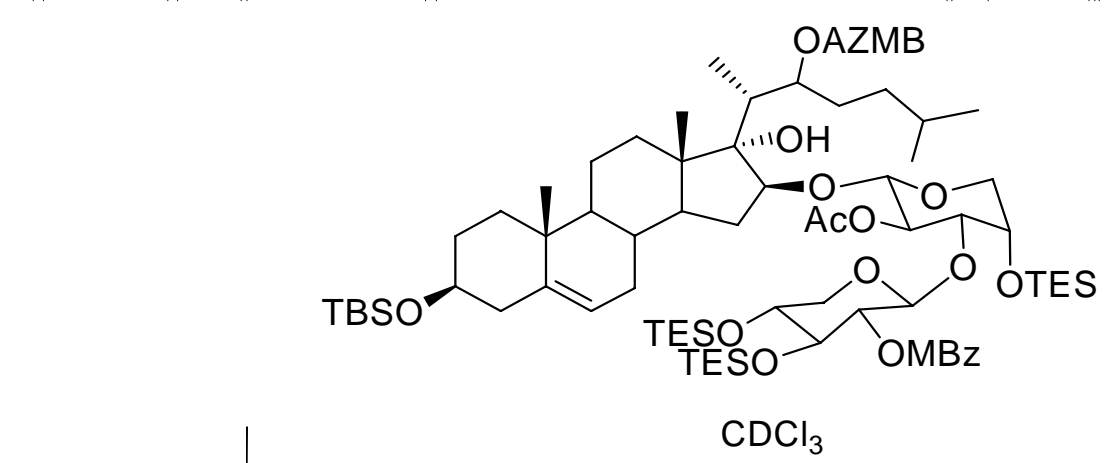




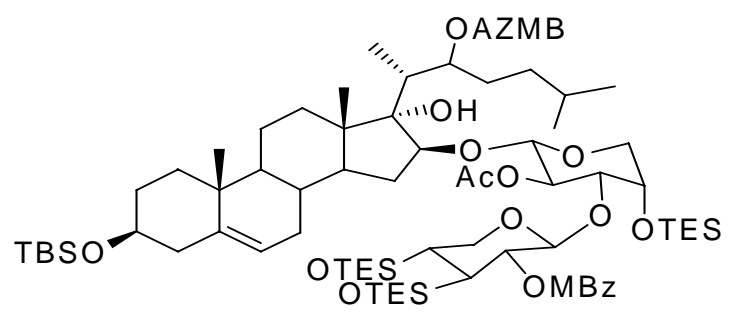

$\mathrm{C}_{79} \mathrm{H}_{131} \mathrm{~N}_{3} \mathrm{O}_{16} \mathrm{Si}_{4}$

Exact Mass: 1489.86
Mol. Wt.: 1491.24

C, $63.63 ; \mathrm{H}, 8.85 ; \mathrm{N}, 2.82 ; \mathrm{O}, 17.17 ; \mathrm{Si}, 7.53$ $\mathrm{CDCl}_{3}$

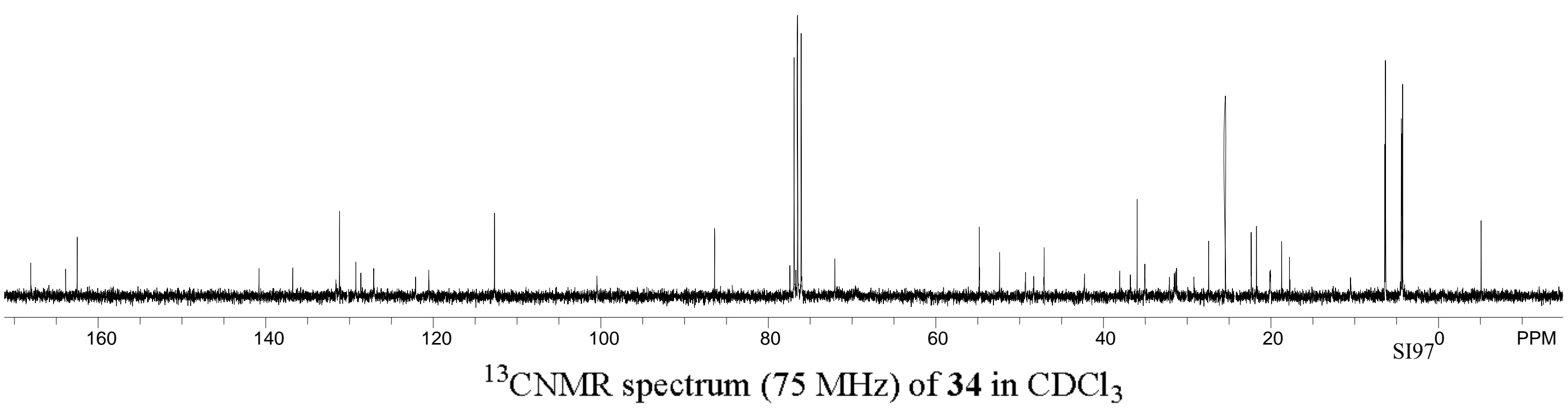




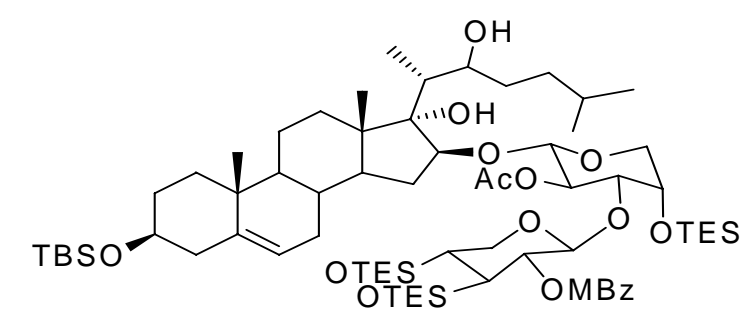

$\mathrm{C}_{71} \mathrm{H}_{126} \mathrm{O}_{15} \mathrm{Si}_{4}$

Exact Mass. 1330.82

C, $64.02 ; \mathrm{H}, 9.53 ; \mathrm{O}, 18.02 ; \mathrm{Si}, 8.43$

$\mathrm{CDCl}_{3}$ 

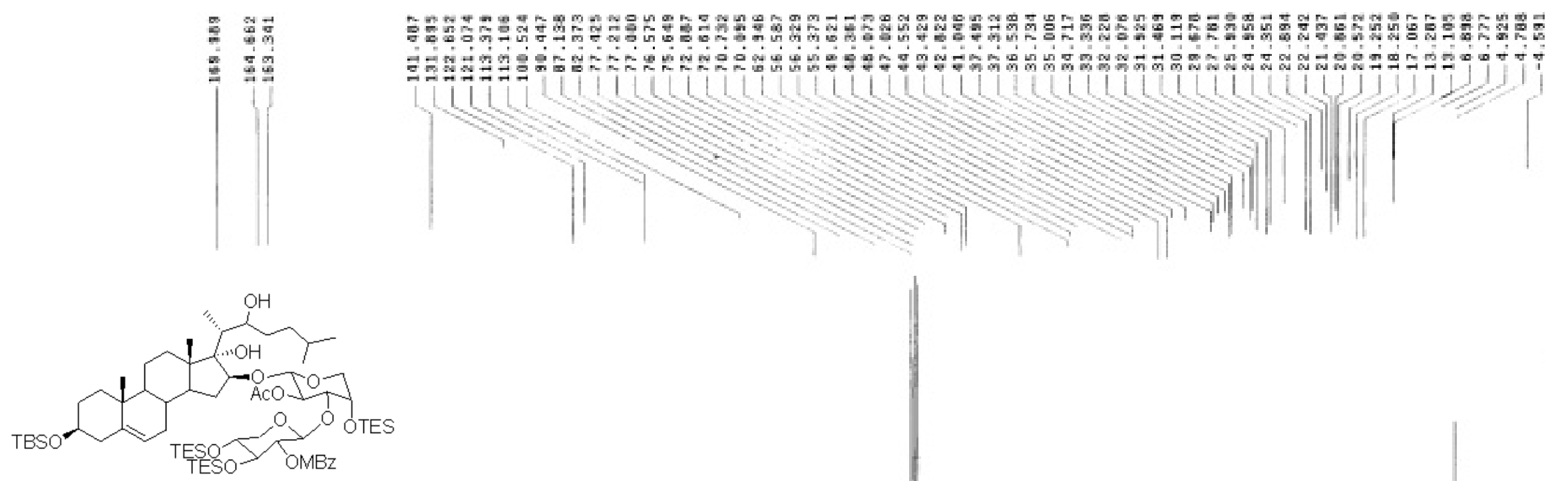

$\mathrm{C}_{71} \mathrm{H}_{126} \mathrm{O}_{15} \mathrm{Si}_{4}$

Exact Mass: 1330.82

Mol. Wt: 1332.09

C. $64.02 ; \mathrm{H}, 9.53 ; \mathrm{O}, 18.02 ; \mathrm{Si}, 8.43$

$\mathrm{CDCl}_{3}$

\begin{tabular}{|c|c|c|c|c|c|c|c|}
\hline 200 & 180 & 160 & 140 & 120 & 100 & 80 & 60 \\
\hline
\end{tabular}



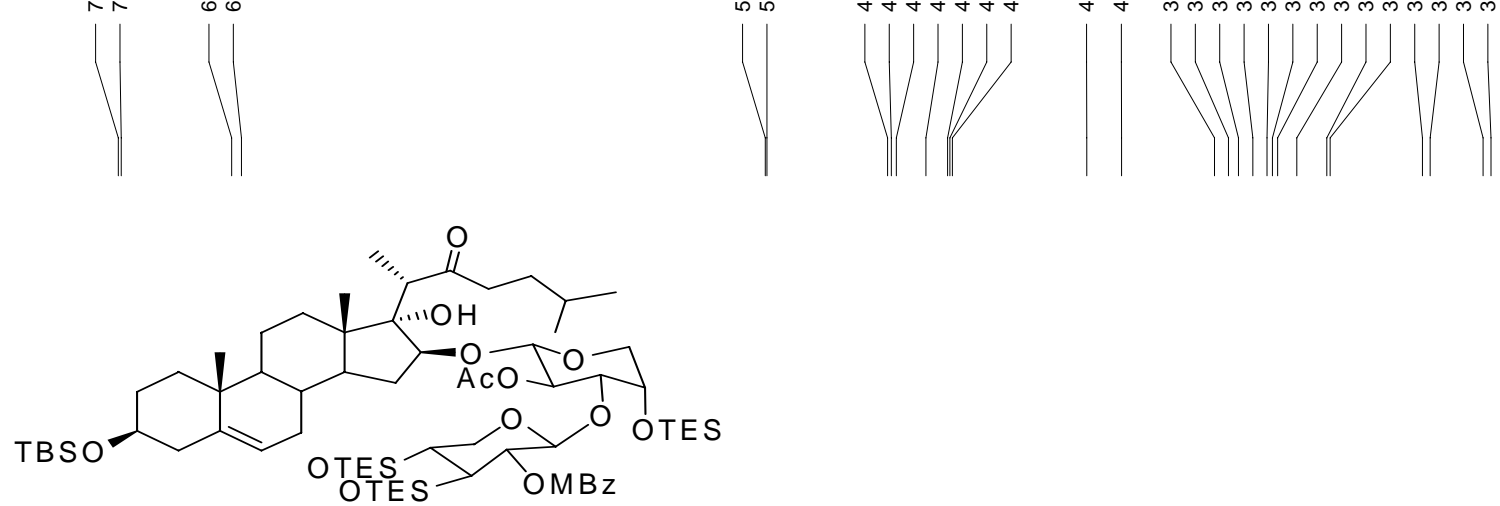

$\mathrm{C}_{71} \mathrm{H}_{124} \mathrm{O}_{15} \mathrm{Si}_{4}$

Exact Mass: 1328.8

Mol. Wt.: 1330.08

C, $64.11 ; \mathrm{H}, 9.40 ; \mathrm{O}, 18.04 ; \mathrm{Si}, 8.45$

$$
\mathrm{CDCl}_{3}
$$



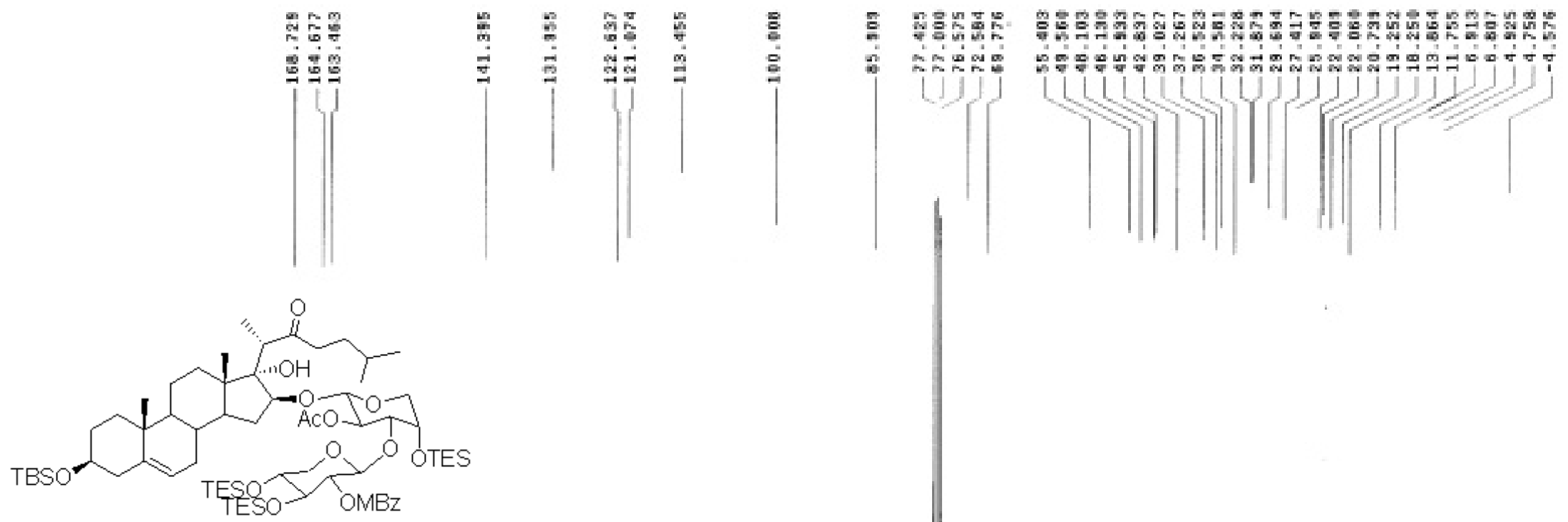

$\mathrm{C}_{71} \mathrm{H}_{124} \mathrm{O}_{15} \mathrm{Si}_{4}$

Exact Mass: 1328.8

Mol. Wt: 1330.08

C. $64.11 ; \mathrm{H}, 9.40 ; \mathrm{O}, 18.04 ; \mathrm{Si}, 8.45$

$\mathrm{CDCl}_{3}$

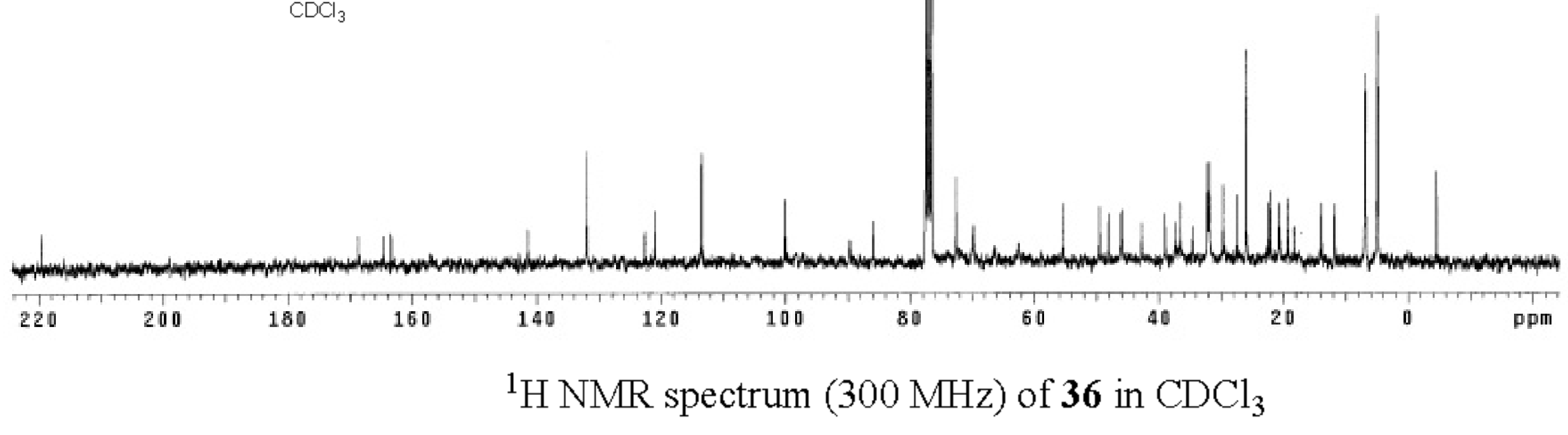




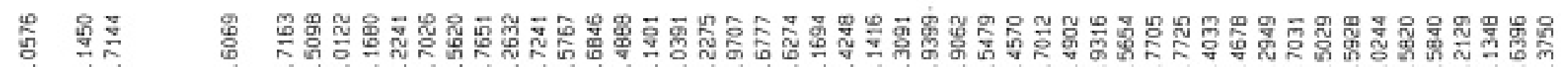

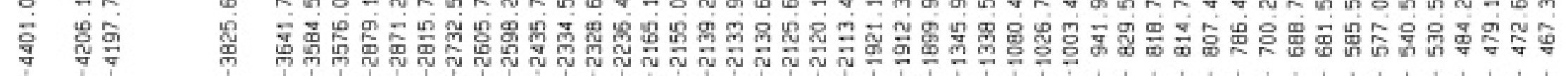

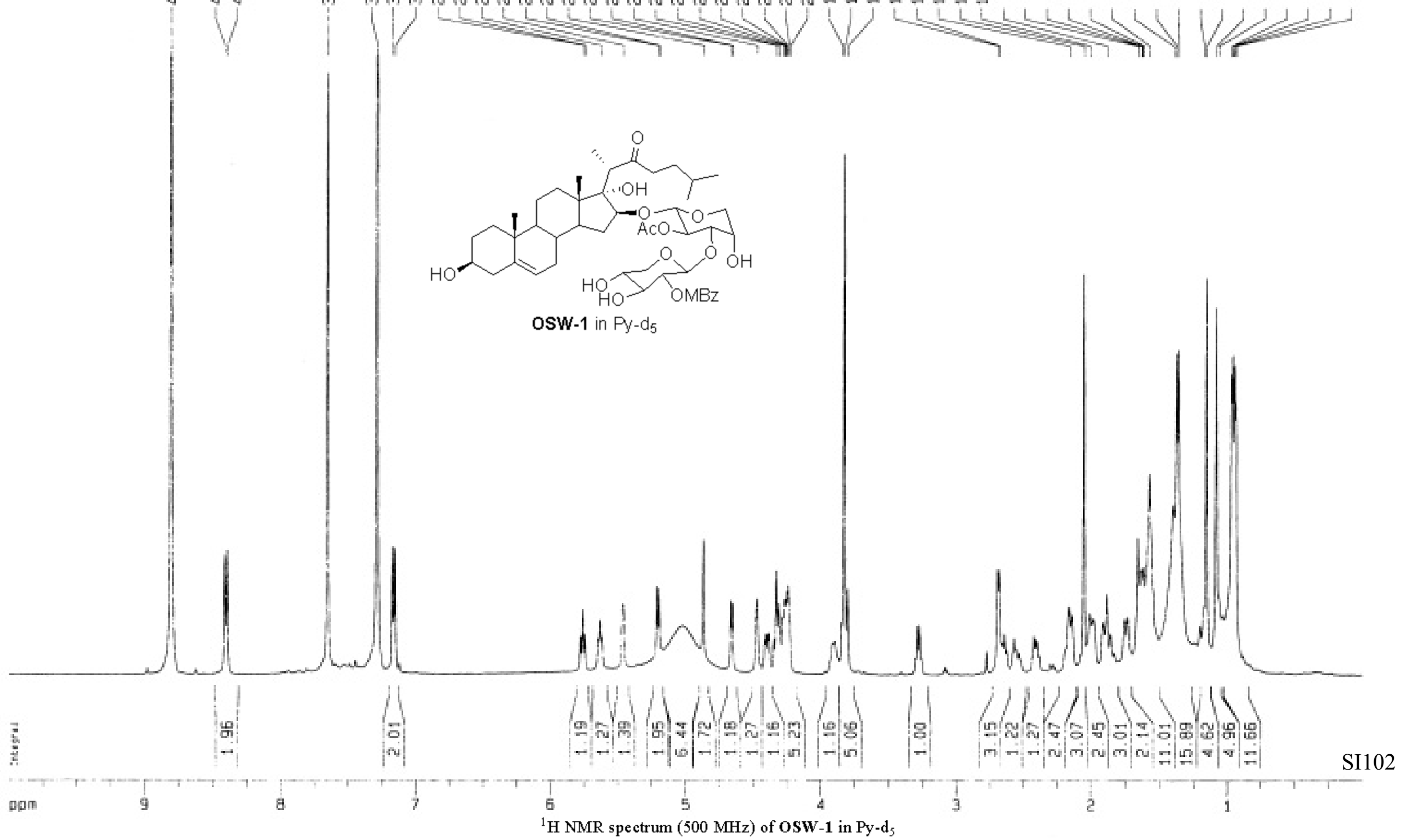




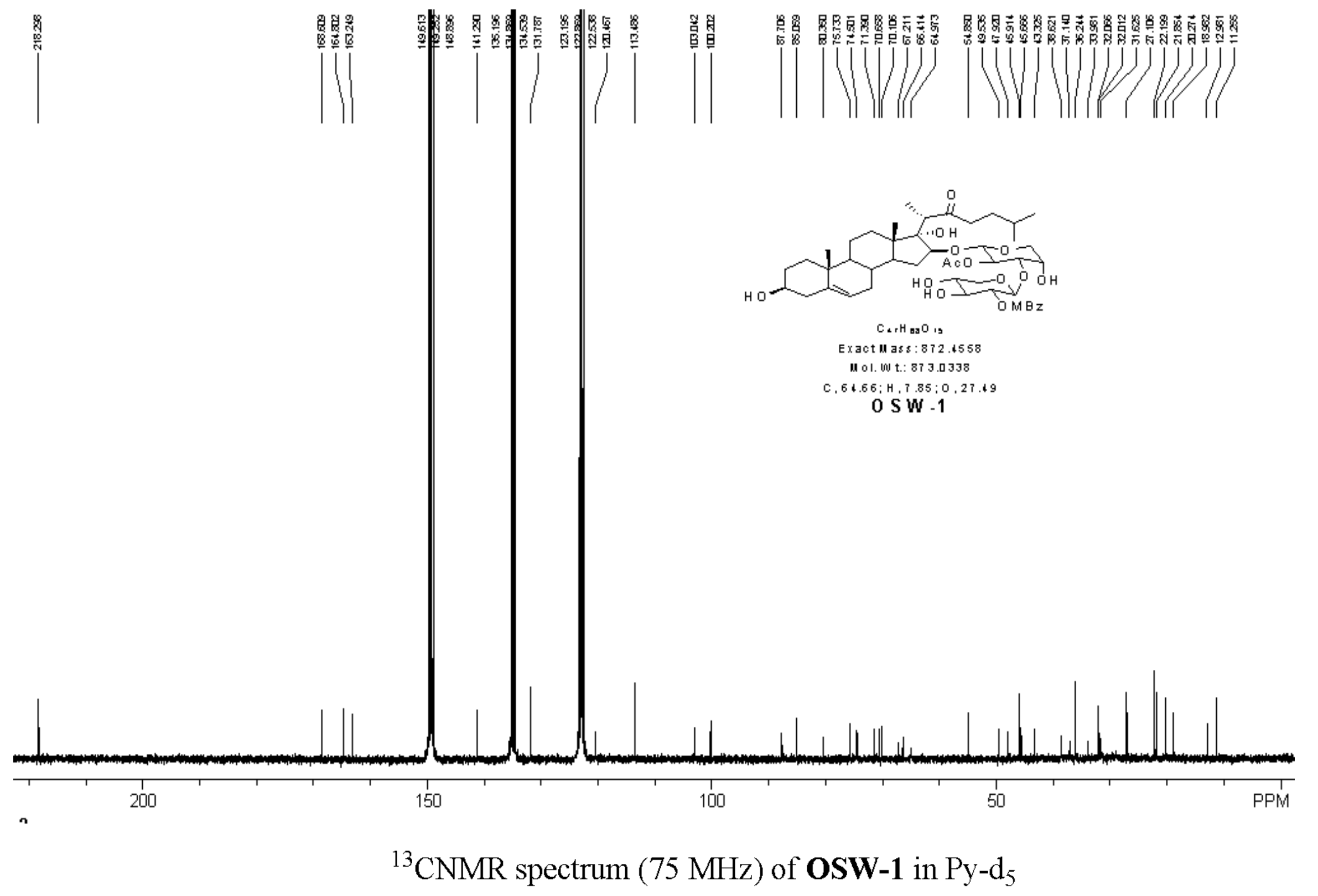




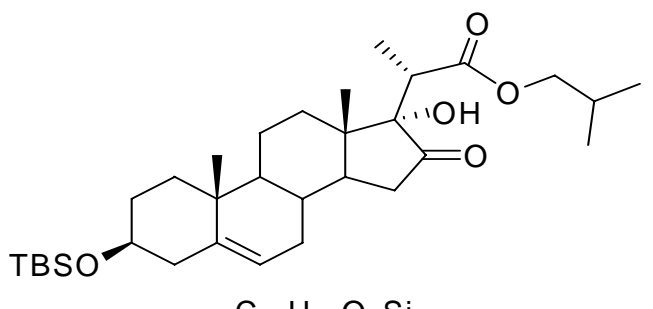

$$
\mathrm{C}_{32} \mathrm{H}_{54} \mathrm{O}_{5} \mathrm{Si}
$$

Exact Mass: 546.37

Mol. Wt.: 546.85

C, $70.28 ; \mathrm{H}, 9.95 ; \mathrm{O}, 14.63 ; \mathrm{Si}, 5.14$

$\mathrm{CDCl}_{3}$ 

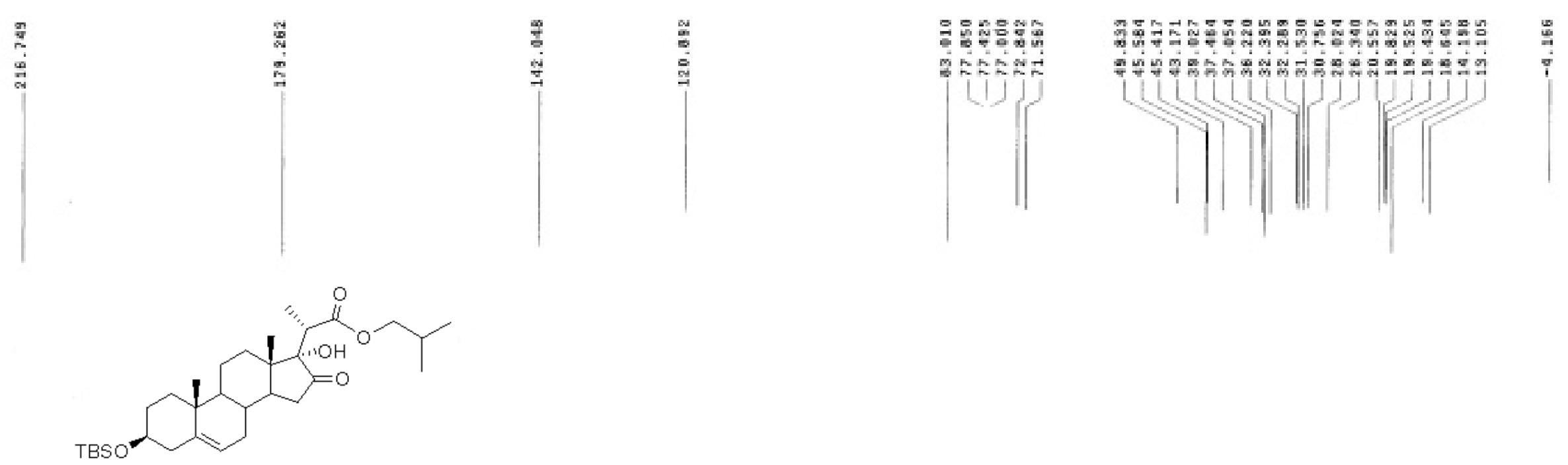

$\mathrm{C}_{32} \mathrm{H}_{54} \mathrm{O}_{5} \mathrm{Si}$
Exact Mass: 546.37

Mol. Wt: 546.85

C. $70.28 ; \mathrm{H}, 9.95 ; \mathrm{O}, 14.63 ; \mathrm{Si}, 5.14$

$\mathrm{CDCl}_{3}$

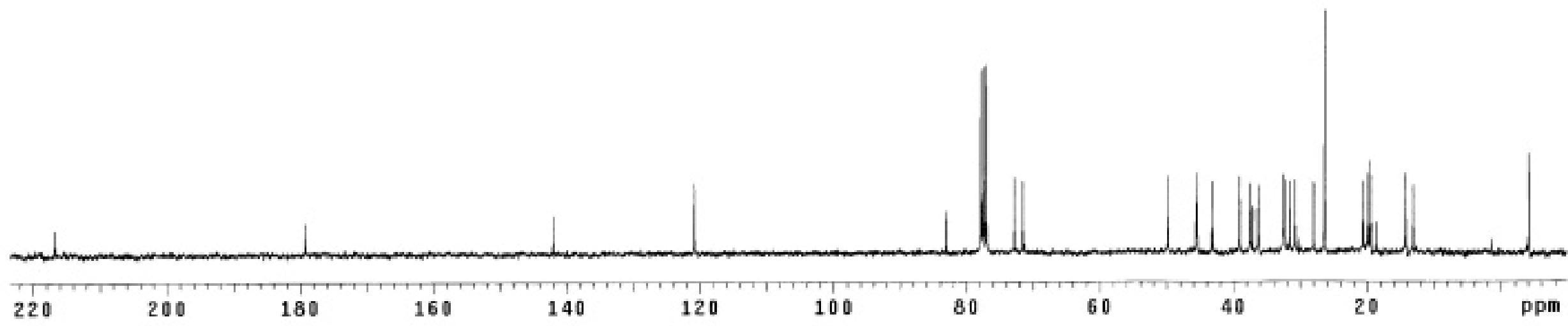

${ }^{1} \mathrm{H}$ NMR spectrum $(300 \mathrm{MHz})$ of $\mathbf{3 7}$ in $\mathrm{CDCl}_{3}$ 


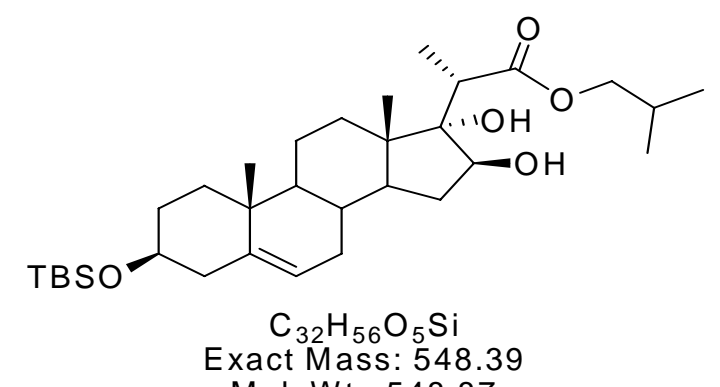

Mol. Wt: 548.87

C, $70.02 ; \mathrm{H}, 10.28 ; \mathrm{O}, 14.57 ; \mathrm{Si}, 5.12$

$$
\mathrm{CDCl}_{3}
$$

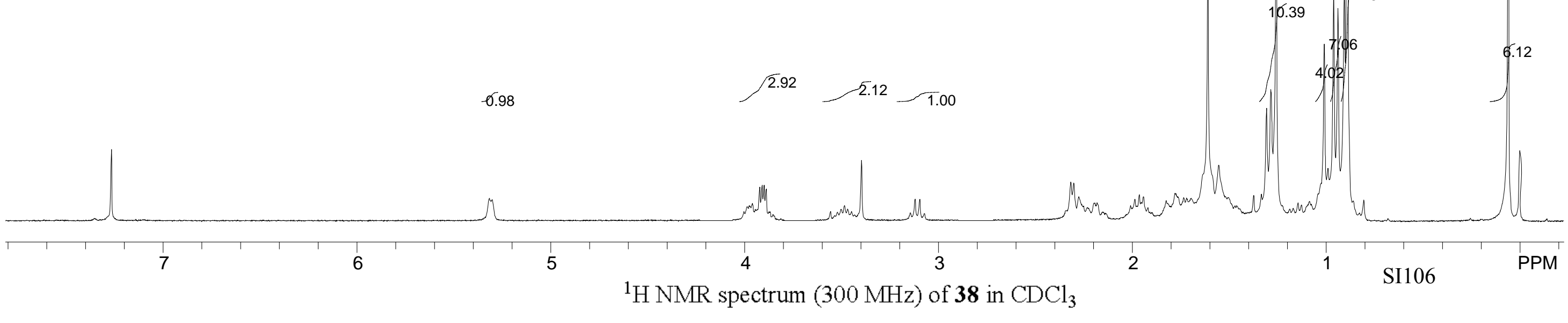



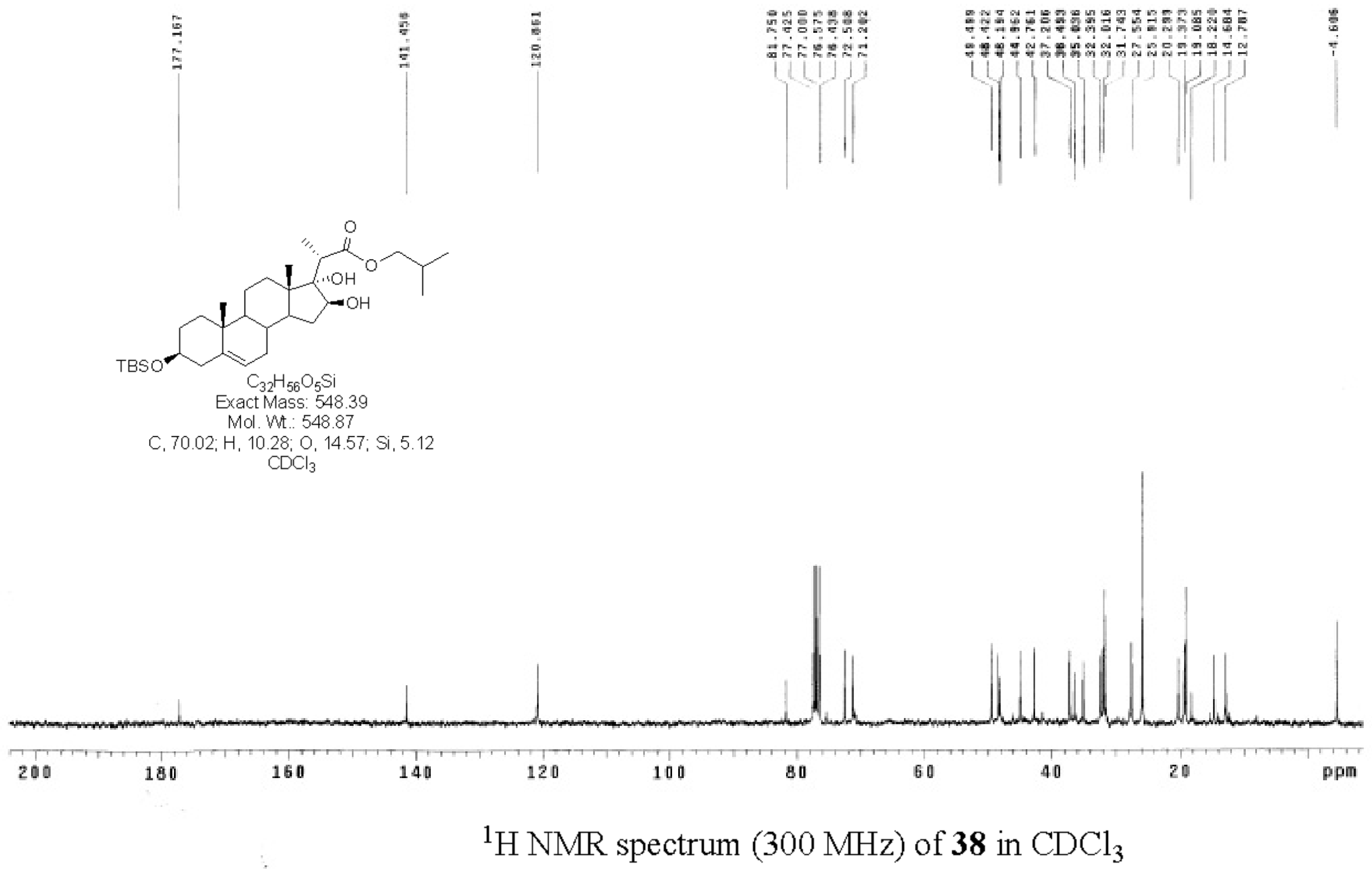


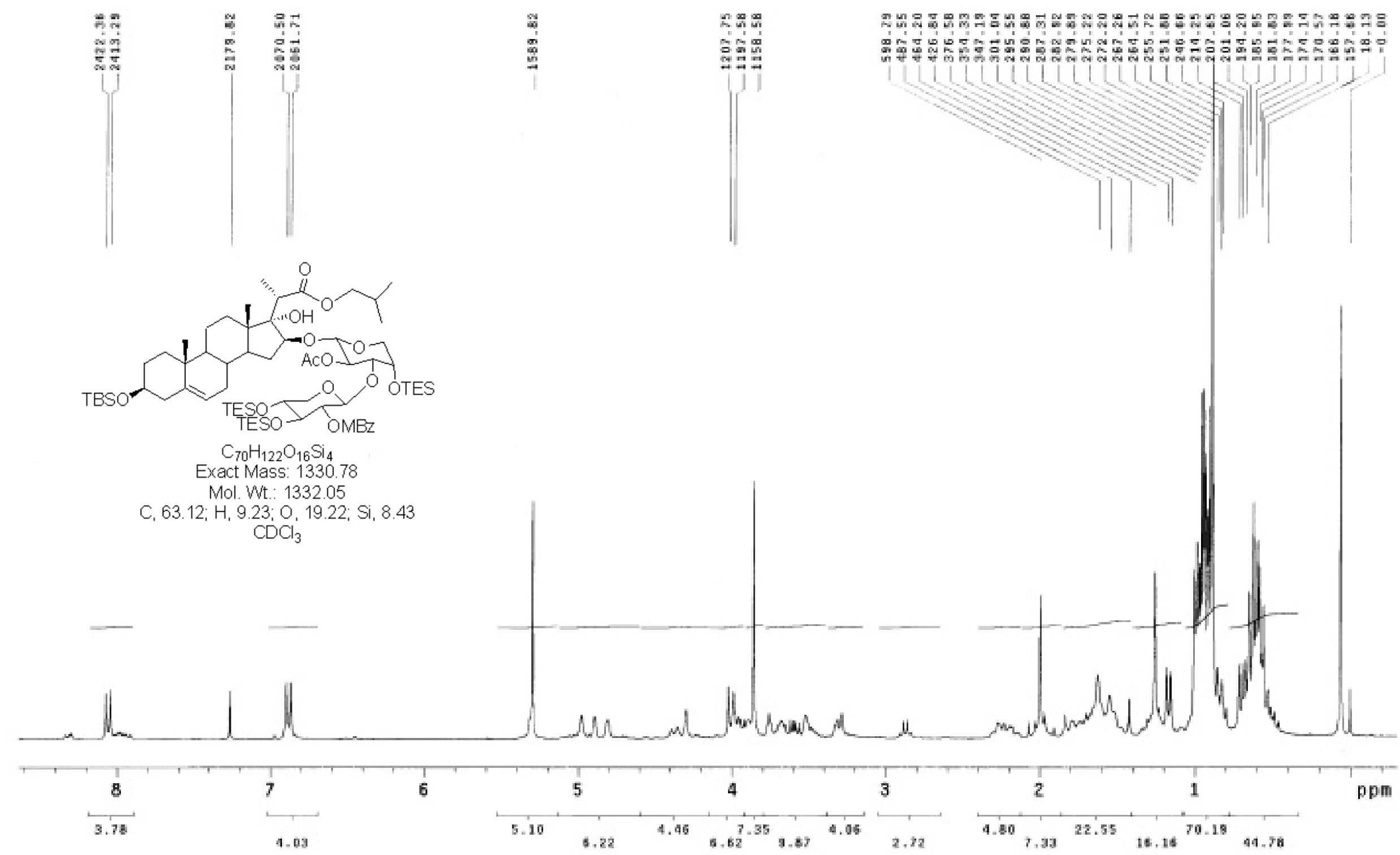

${ }^{1} \mathrm{H} \mathrm{NMR}$ spectrum $\left(300 \mathrm{MHz}\right.$ ) of $\mathbf{3 9}$ in $\mathrm{CDCl}_{3}$ 
c13

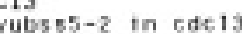

Pulse sequence: 52 pul
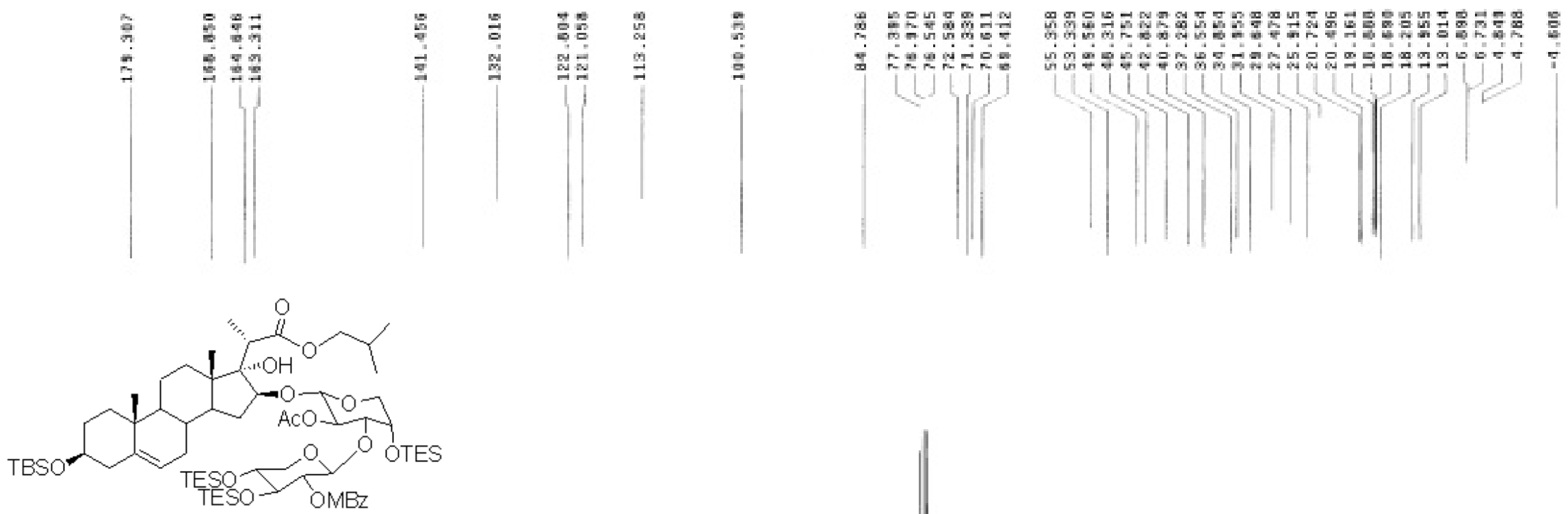

$\mathrm{C}_{70} \mathrm{H}_{122} \mathrm{O}_{16} \mathrm{Si}_{4}$

Exact Mass: 1330.78

Mol. Wt: 1332.05

C. $63.12 ; H, 9.23 ; 0,19.22 ; \mathrm{Si}, 8.43$

$\mathrm{CDCl}_{3}$

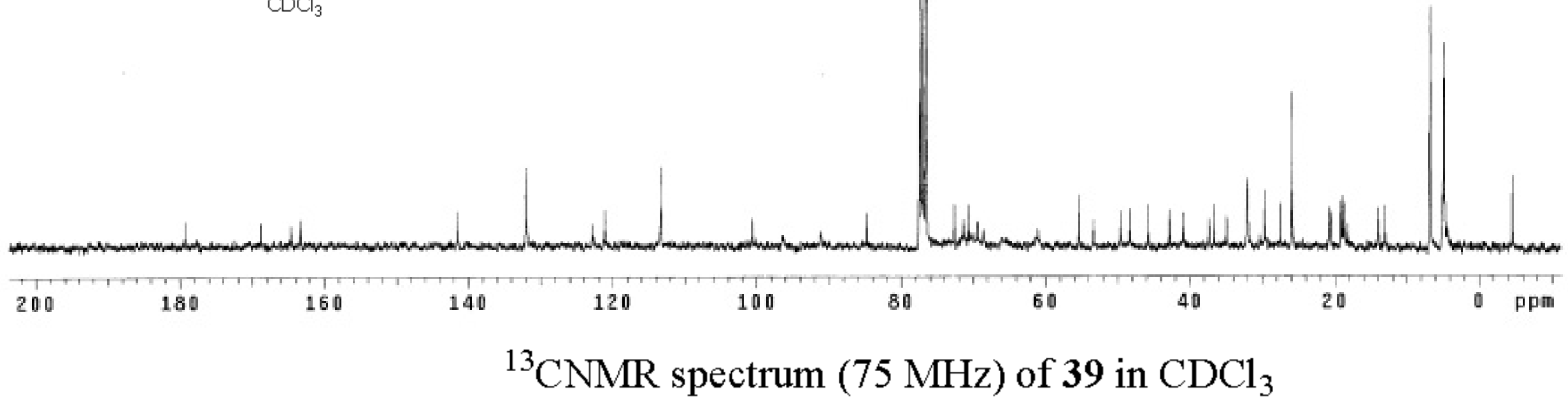



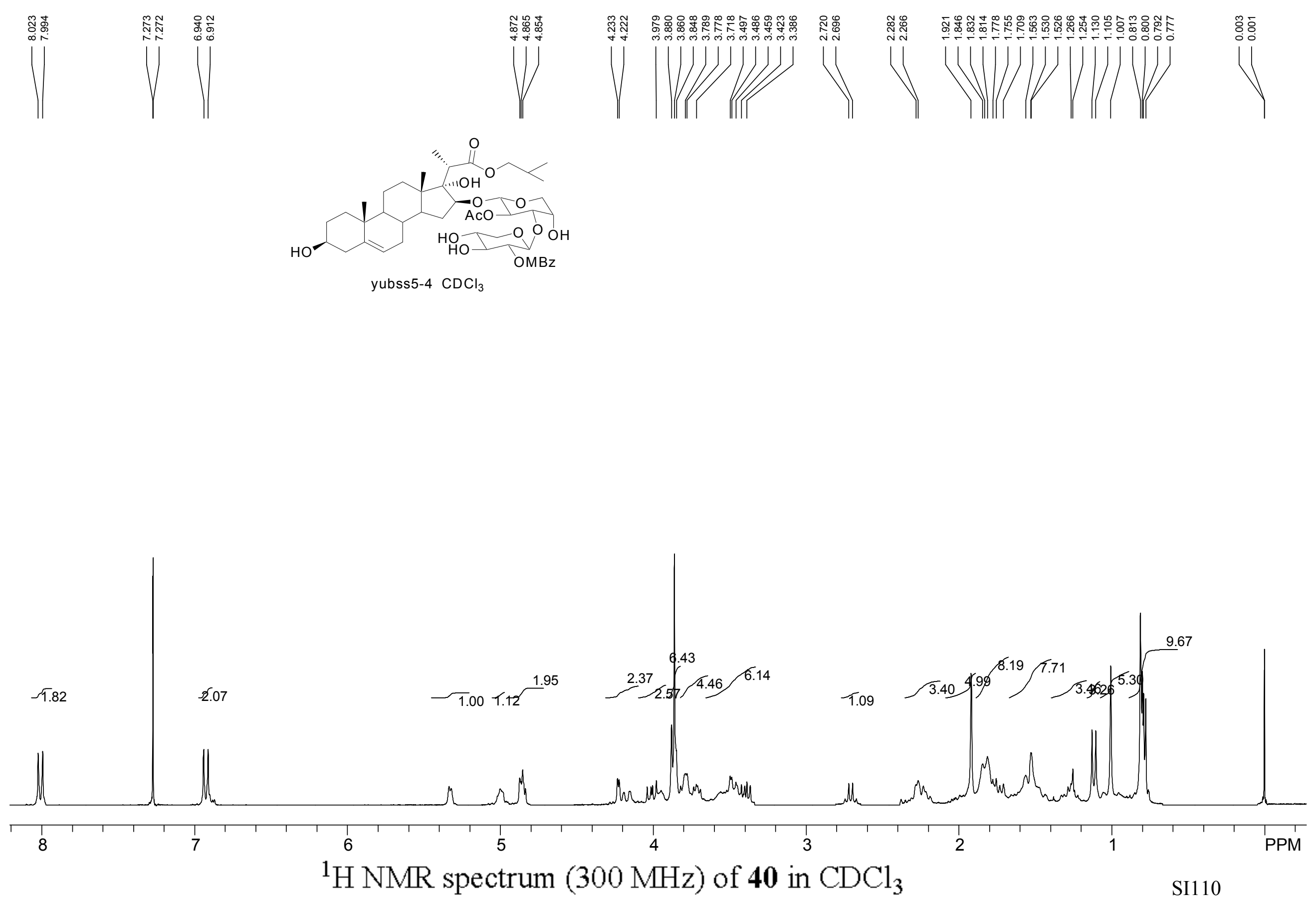


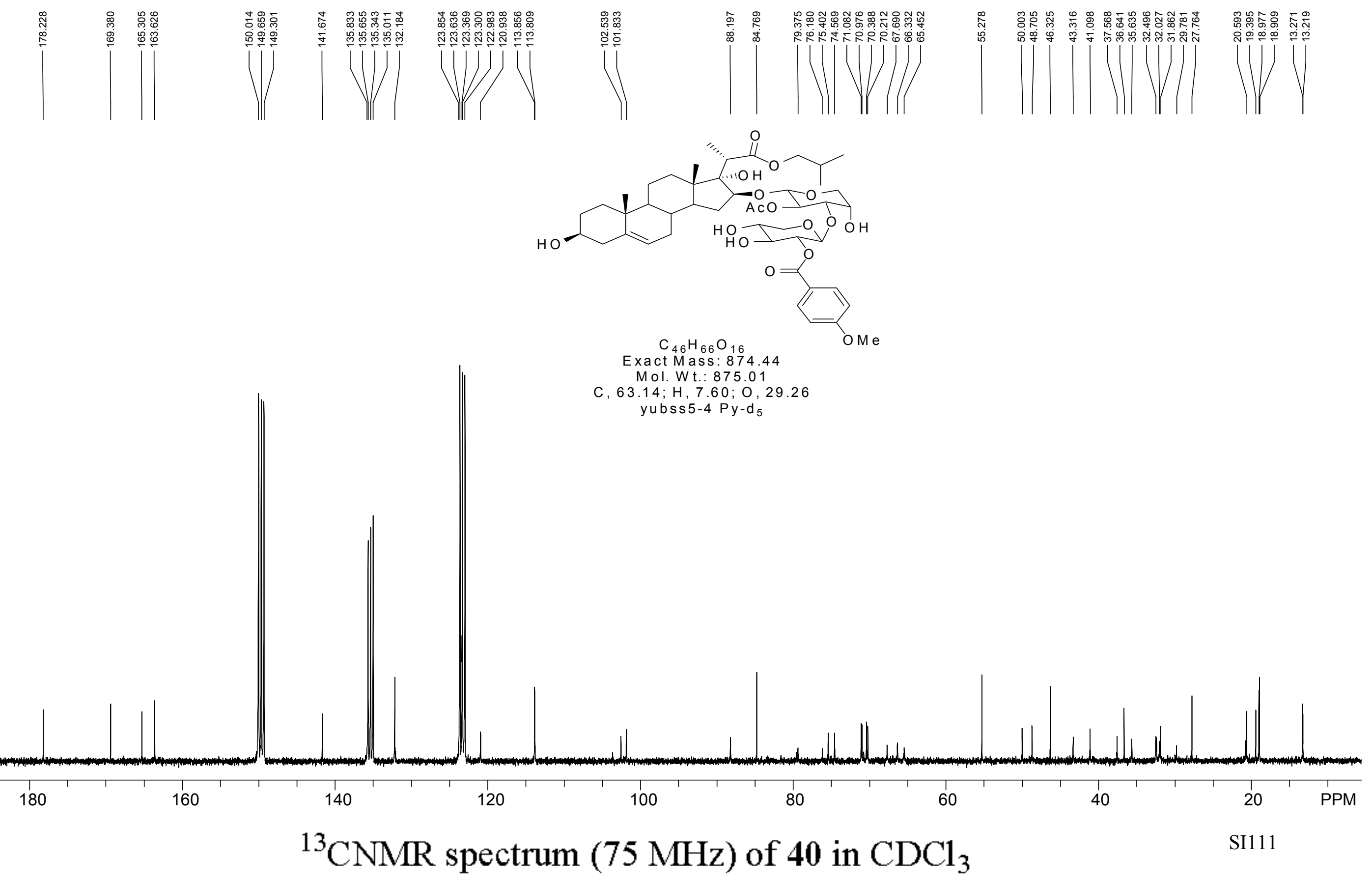




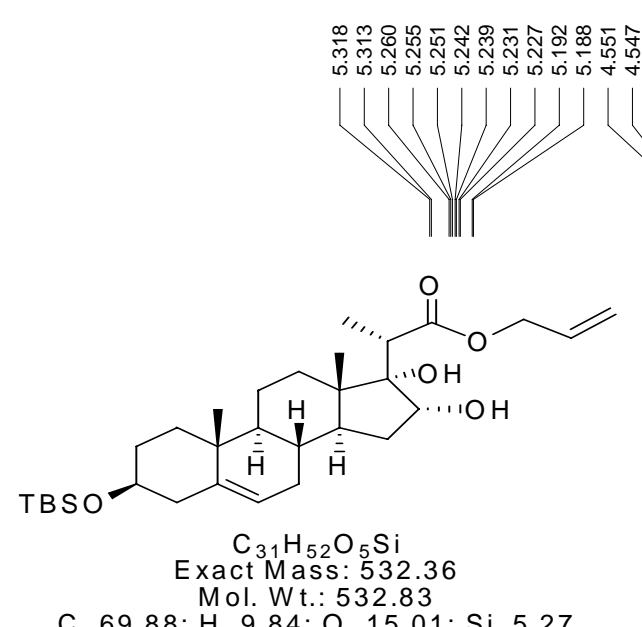

C, $69.88 ; \mathrm{H}, 9.84 ; \mathrm{O}, 15.01 ; \mathrm{Si}, 5.27$

10.45 


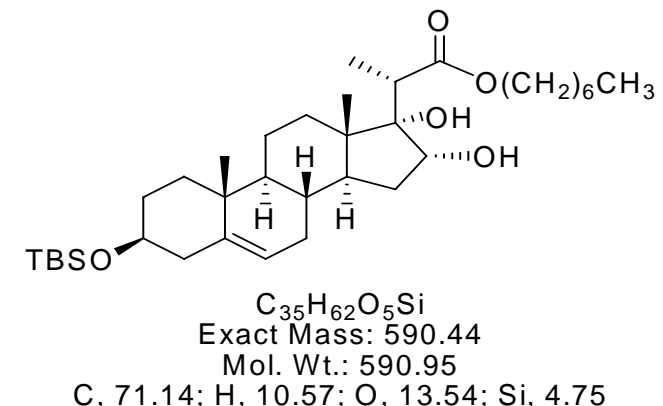

C, $71.14 ; \mathrm{H}, 10.57 ; \mathrm{O}, 13.54 ; \mathrm{Si}, 4.75$ 
c13

yutes-125 in $t d d \in 1$.

Pulse Sequence: szput
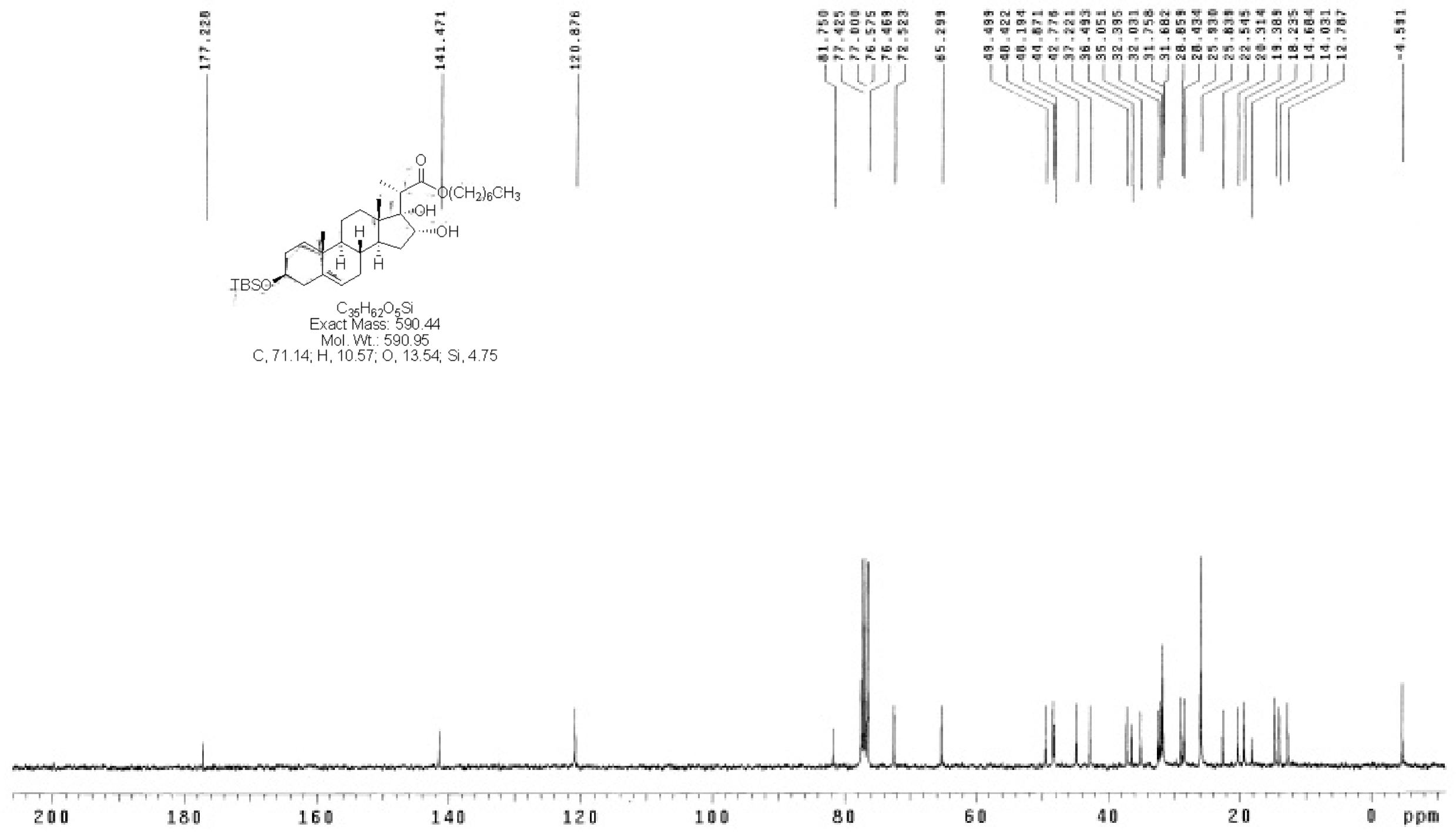

${ }^{13} \mathrm{CNMR}$ spectrum $\left(75 \mathrm{MHz}\right.$ ) of 42 in $\mathrm{CDCl}_{3}$ 


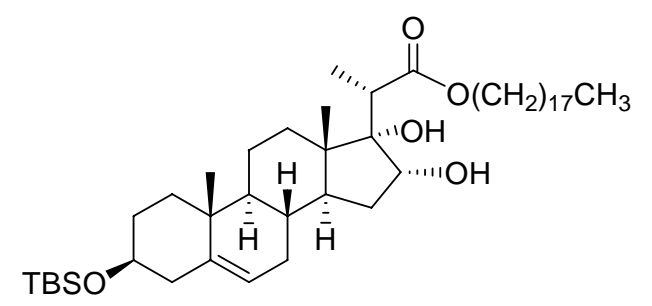

$\mathrm{C}_{46} \mathrm{H}_{84} \mathrm{O}_{5} \mathrm{Si}$

Exact Mass: 744.61

Mol. Wt.: 745.24

C, 74.14; H, 11.36; O, 10.73; Si, 3.77 

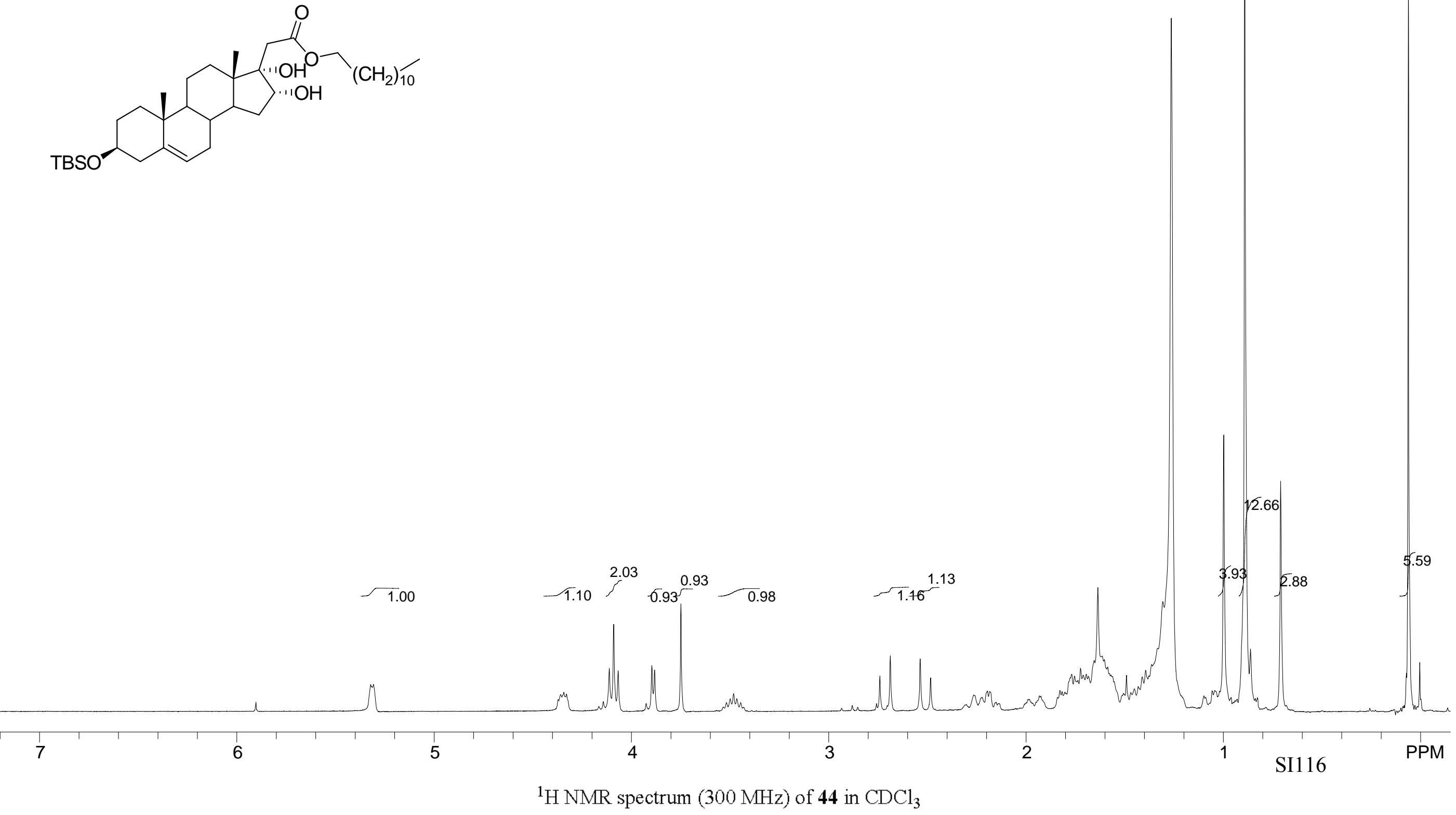


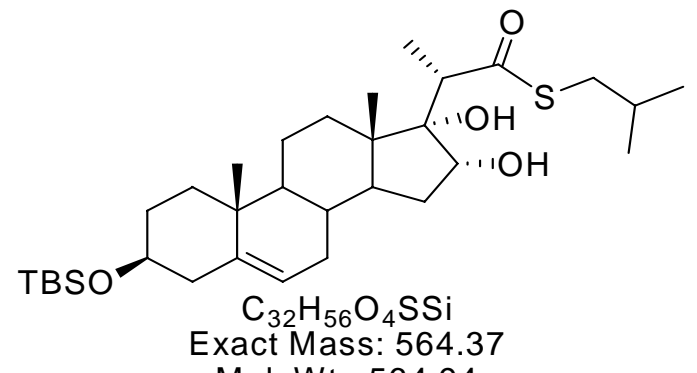

C, $68.03 ; \mathrm{H}, 9.99 ; \mathrm{O}, 11.33 ; \mathrm{S}, 5.68 ; \mathrm{Si}, 4.97$ $\mathrm{CDCl}_{3}$

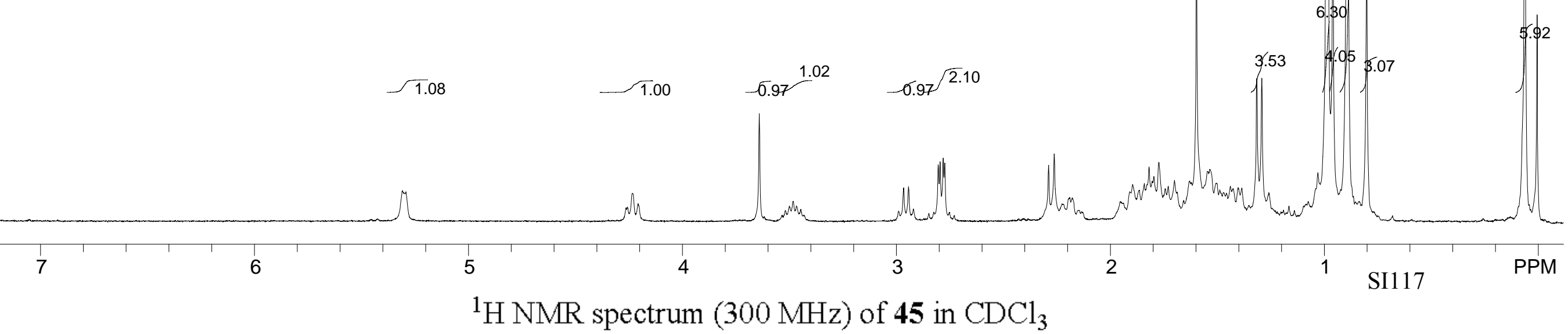



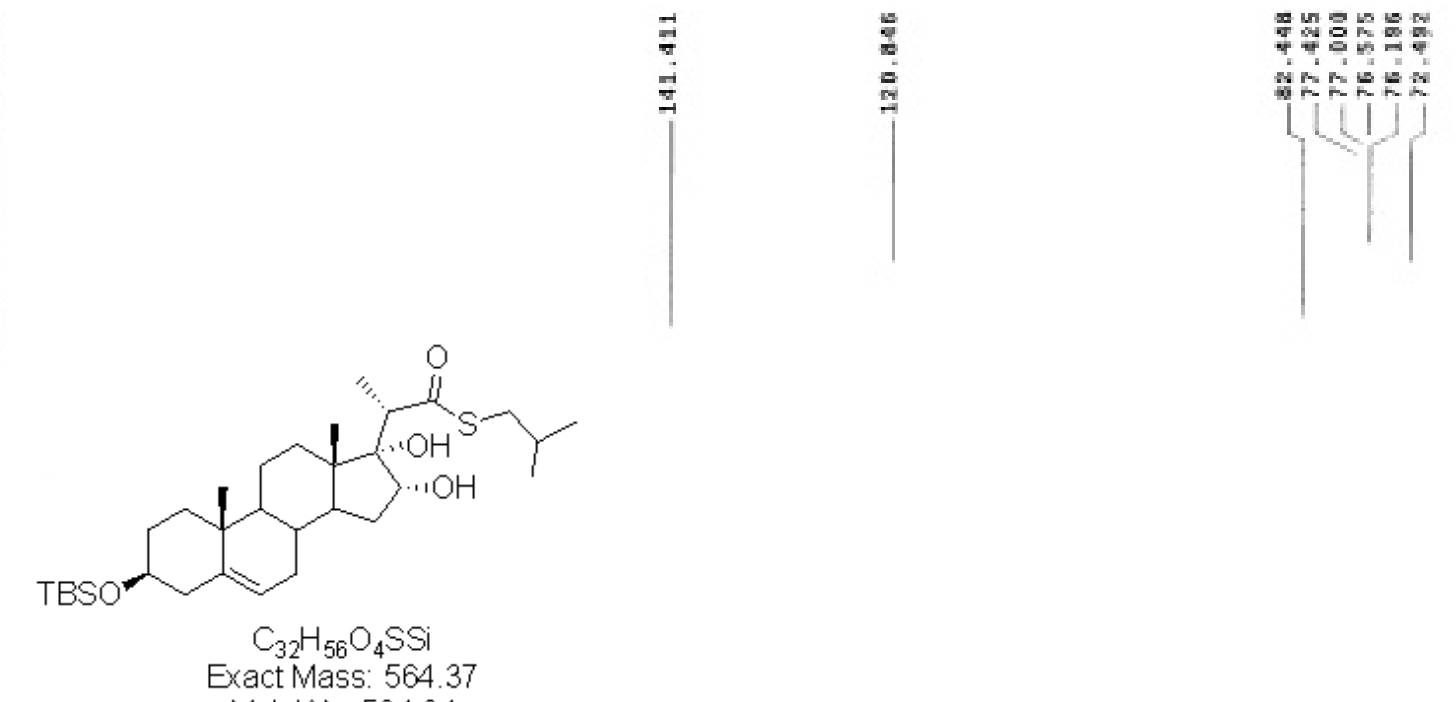

Mol Mt. 564.94

C. $68.03 ; \mathrm{H}, 9.99 ; 0,11.33 ; \mathrm{S}, 5.68 ; \mathrm{Si}, 4.97$

$\mathrm{CDCl}_{3}$

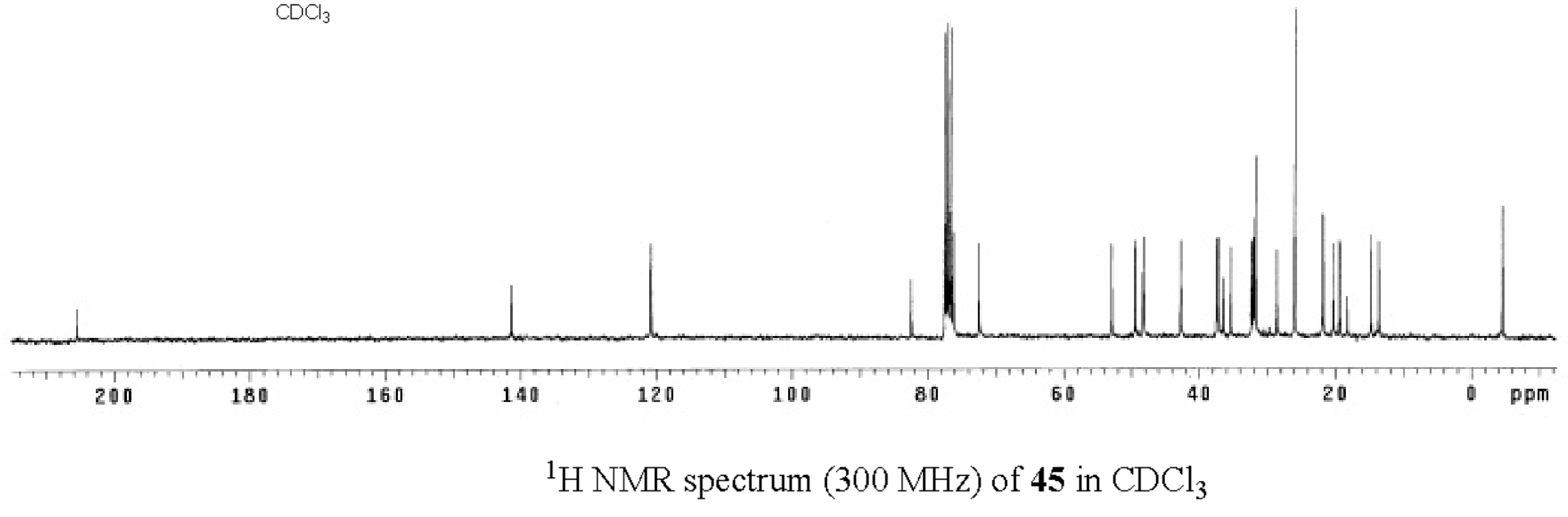




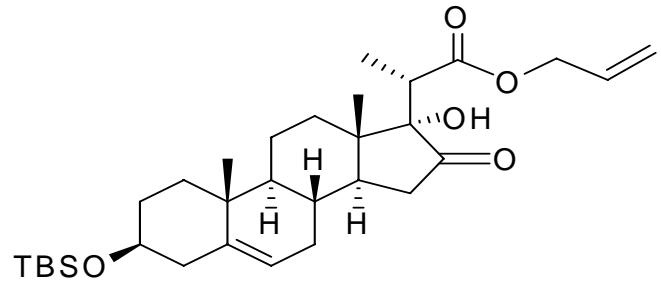

$\mathrm{C}_{31} \mathrm{H}_{50} \mathrm{O}_{5} \mathrm{Si}$

Exact Mass: 530.34

Mol. Wt.: 530.81

C , $70.14 ; \mathrm{H}, 9.49: 0,15.07 \cdot \mathrm{Si}, 5.29$

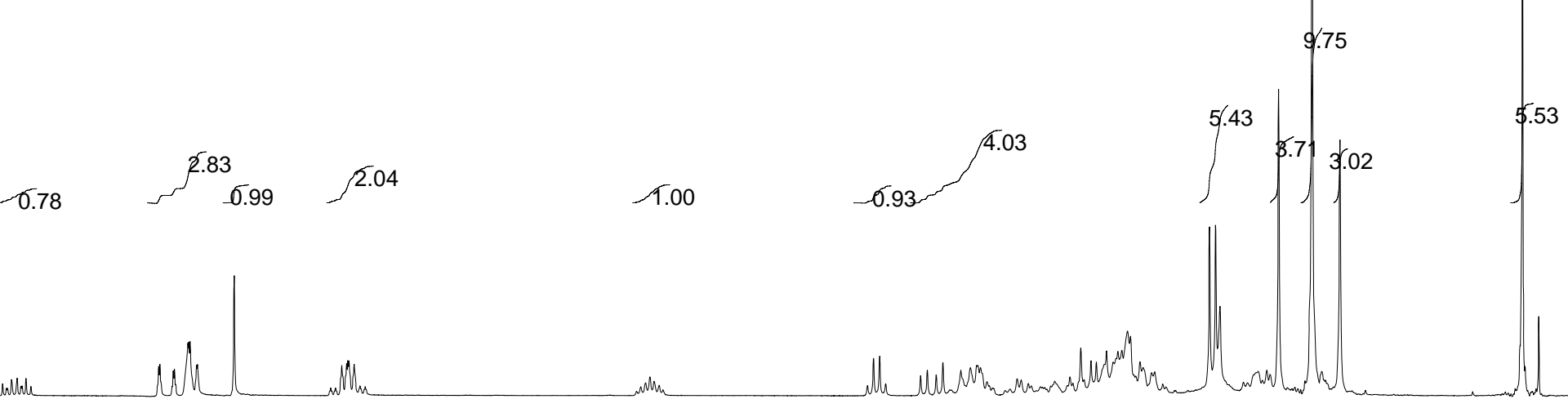




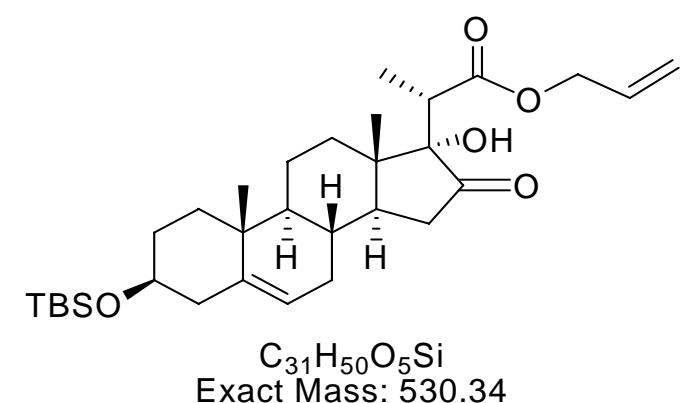

Exact Mass: 530.34

Mol. Wt: 530.81

C, $70.14 ; \mathrm{H}, 9.49 ; \mathrm{O}, 15.07 ; \mathrm{Si}, 5.29$ 


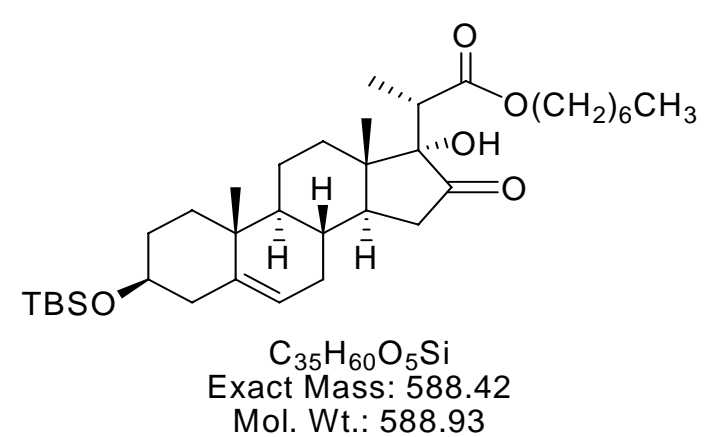

C, 71.38; H, 10.27; O, 13.58; Si, 4.77 
yubt $3-127$ in cde 13

Pulse Sequencel szpul
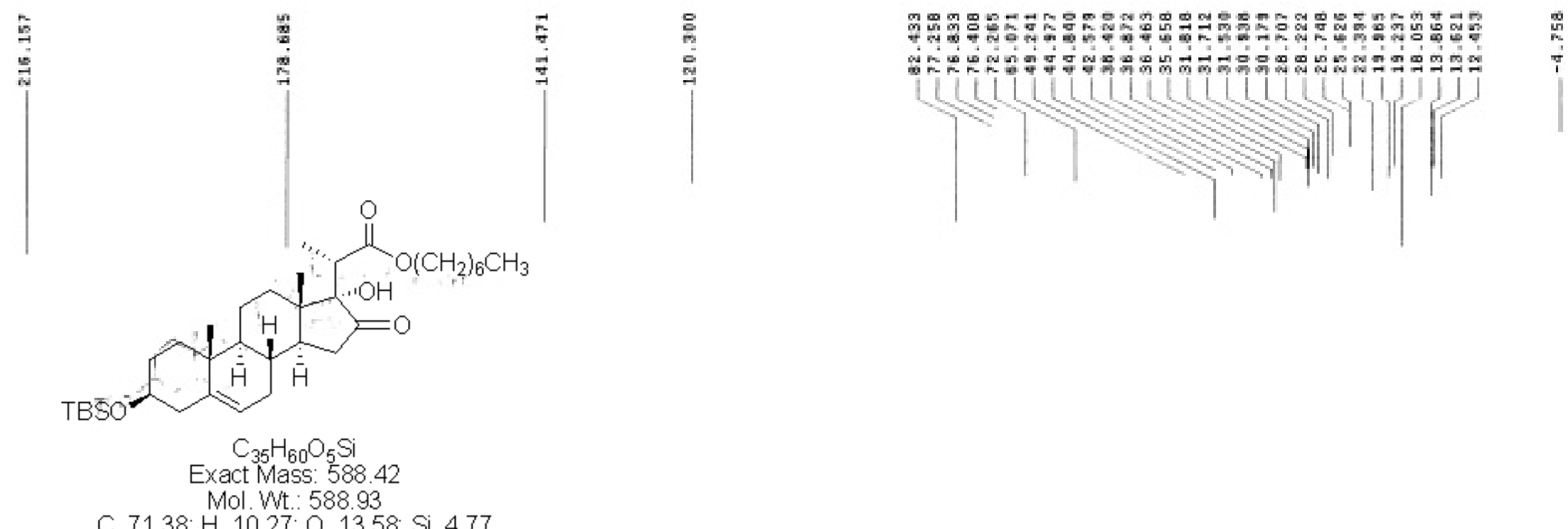

C. $71.38 ; \mathrm{H}, 10.27 ; \mathrm{O}, 13.58 ; \mathrm{Si}, 4.77$

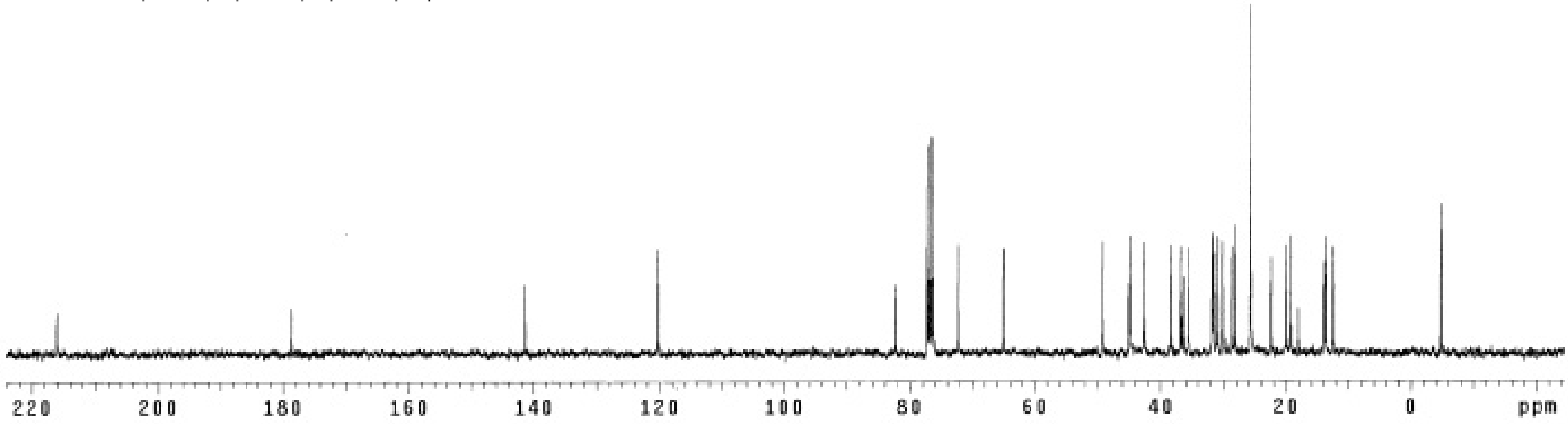

${ }^{13} \mathrm{CNMR}$ spectrum $(75 \mathrm{MHz})$ of 48 in $\mathrm{CDCl}_{3}$ 


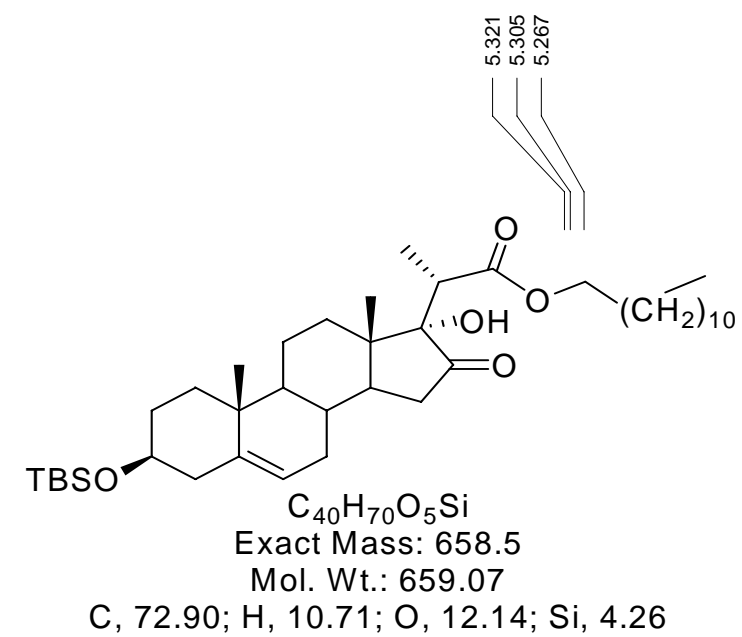

C, $72.90 ; \mathrm{H}, 10.71 ; \mathrm{O}, 12.14 ; \mathrm{Si}, 4.26$

$$
\mathrm{CDCl}_{3}
$$

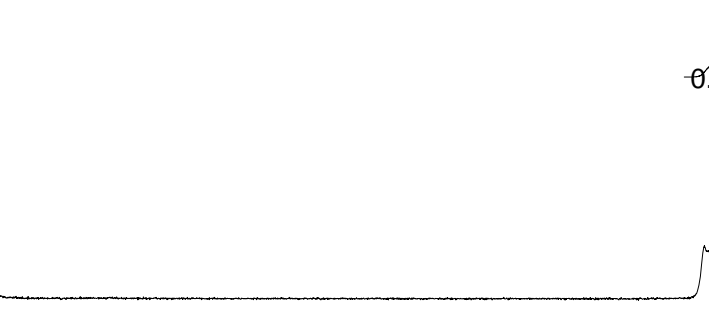

$$
\frac{1.03}{0.86}
$$

2.70 

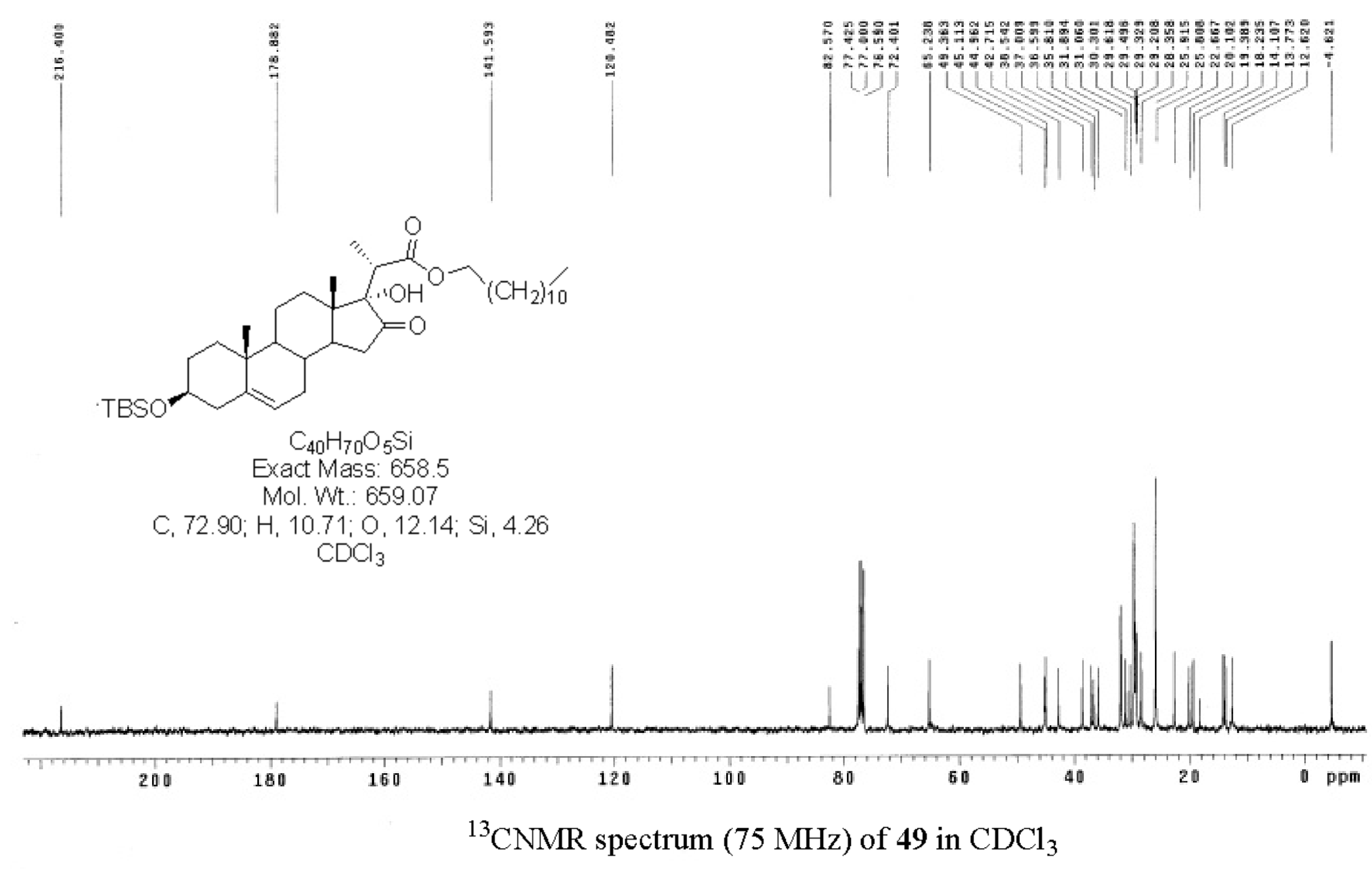


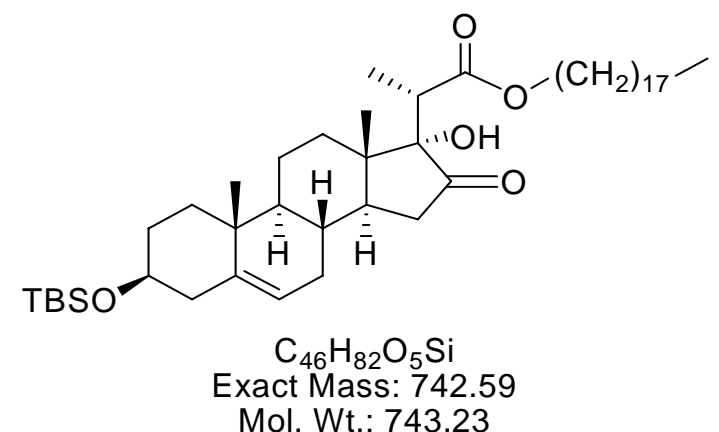

C, 74.34; H, 11.12; O, 10.76; Si, 3.78 


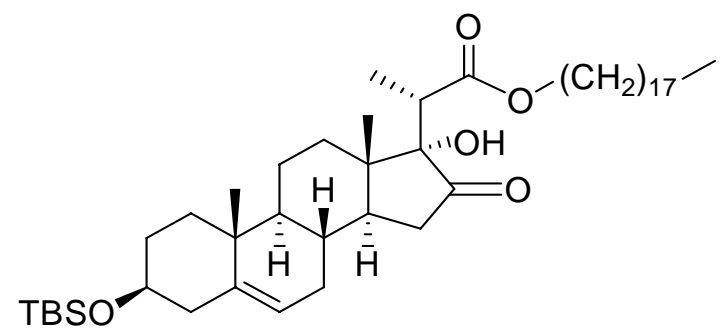

$\mathrm{C}_{46} \mathrm{H}_{82} \mathrm{O}_{5} \mathrm{Si}$

Exact Mass: 742.59

$$
\text { Mol. Wt.: } 743.23
$$

C, $74.34 ; \mathrm{H}, 11.12 ; \mathrm{O}, 10.76 ; \mathrm{Si}, 3.78$

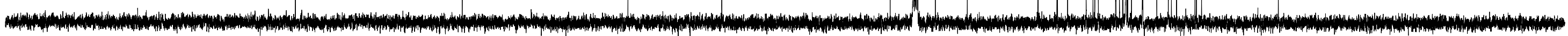




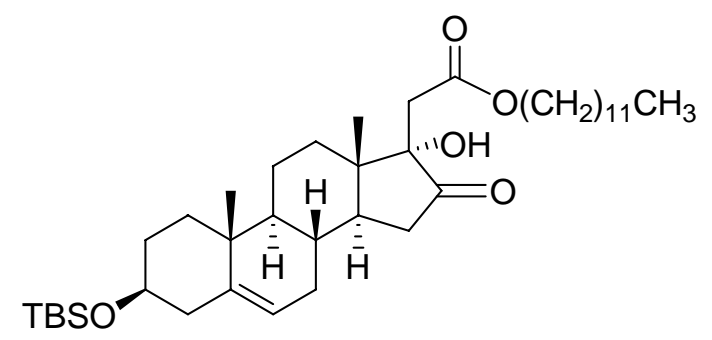

$\mathrm{C}_{39} \mathrm{H}_{68} \mathrm{O}_{5} \mathrm{Si}$

Exact Mass: 644.48

Mol. Wt: 645.04

C, $72.62 ; \mathrm{H}, 10.63 ; \mathrm{O}, 12.40 ; \mathrm{Si}, 4.35$ 

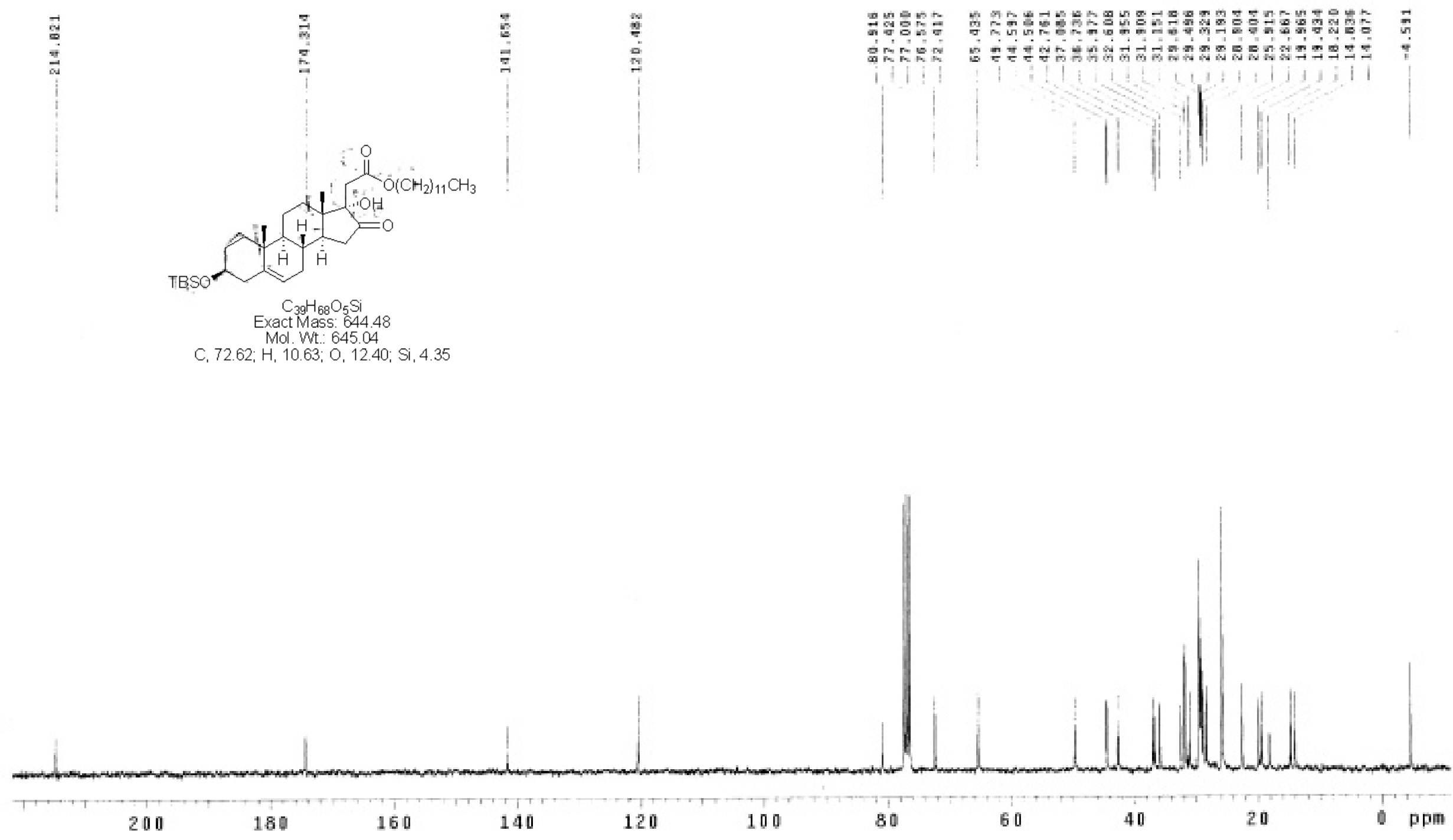

${ }^{13} \mathrm{CNMR}$ spectrum $(75 \mathrm{MHz})$ of 51 in $\mathrm{CDCl}_{3}$ 

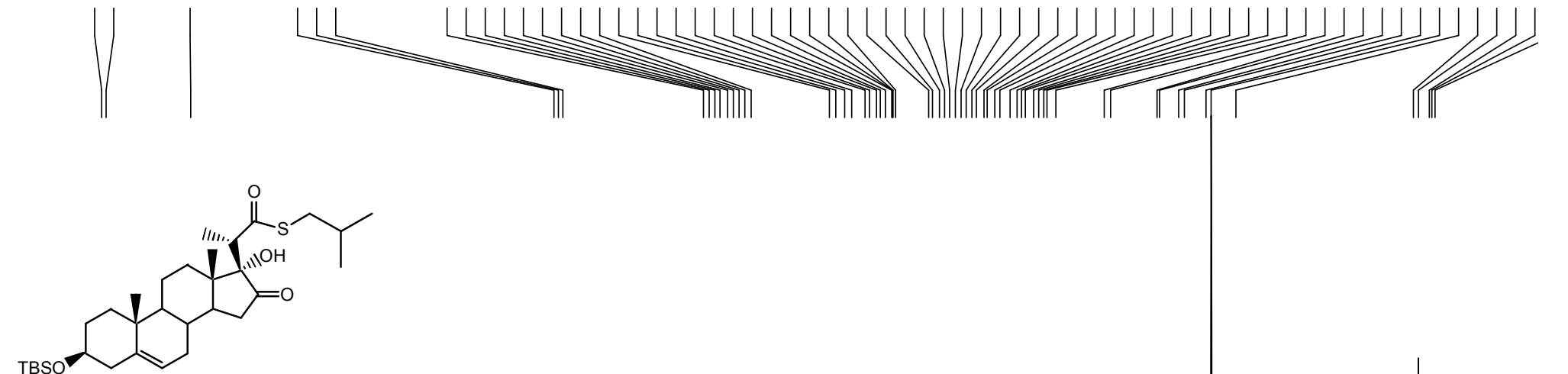

$\mathrm{C}_{32} \mathrm{H}_{54} \mathrm{O}_{4} \mathrm{SSi}$

Exact Mas:

Mol. Wt.: 562.9203

C, $68.28 ; \mathrm{H}, 9.67 ; \mathrm{O}, 11.37 ; \mathrm{S}, 5.70 ; \mathrm{Si}, 4.99$

$\mathrm{SBF}^{-114} \mathrm{CDCl}_{3}$ 
C13 13 subs5-1A in CaCls

Pulse Sequence: s2pul
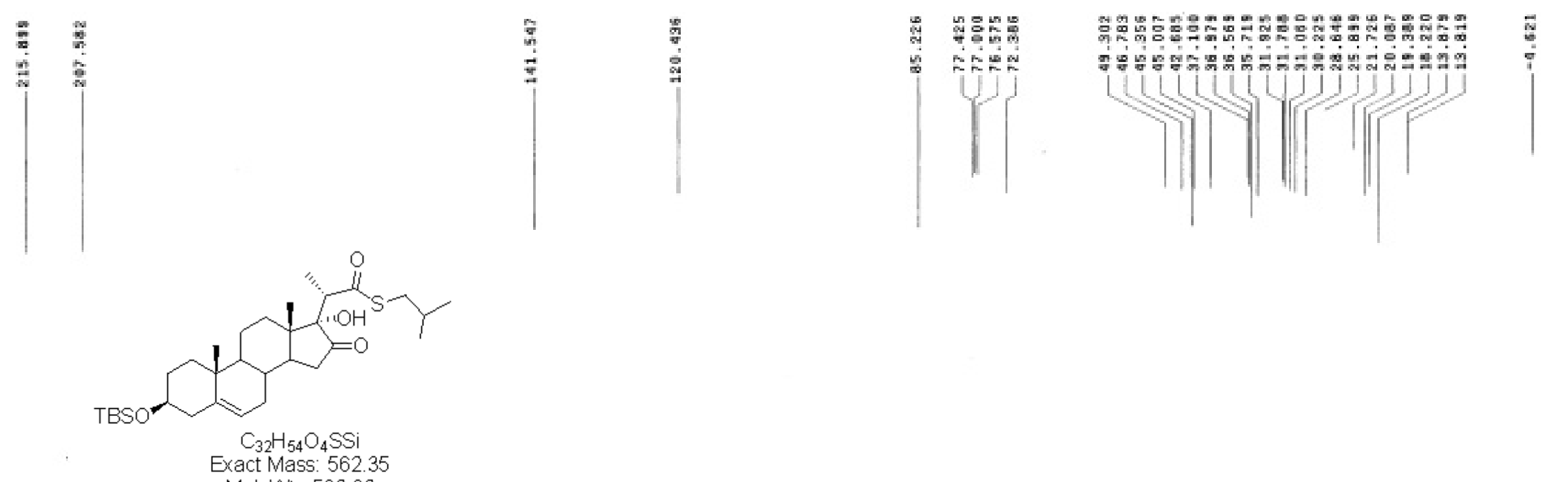

Mol. Wt.: 562.92

C. $68.28 ; \mathrm{H}, 9.67 ; 0,11.37 ; \mathrm{S}, 5.70 ; \mathrm{Si}, 4.99$

$$
\mathrm{CDCl}_{3}
$$

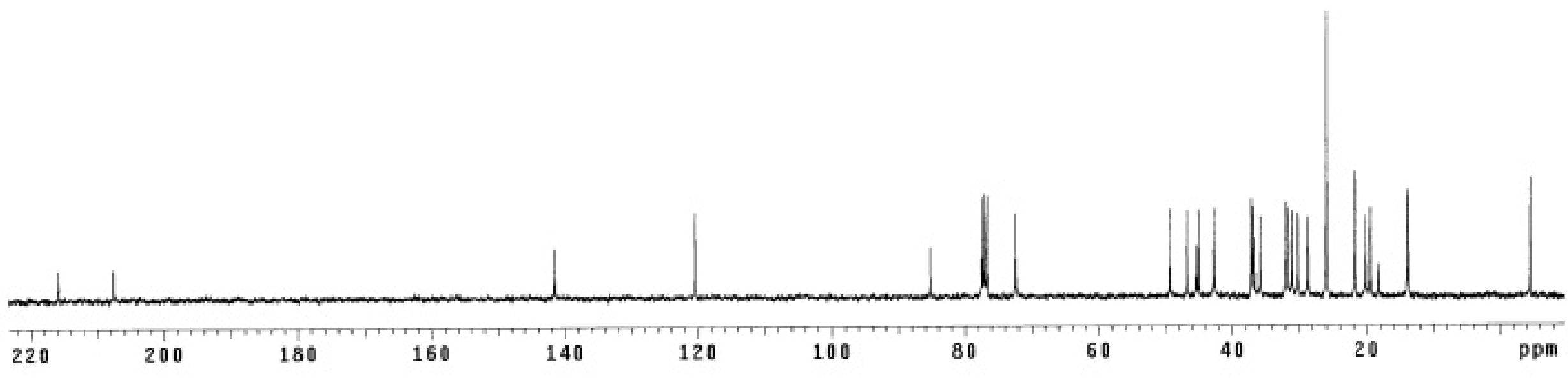

${ }^{13} \mathrm{CNMR}$ spectrum $(75 \mathrm{MHz})$ of 52 in $\mathrm{CDCl}_{3}$ 


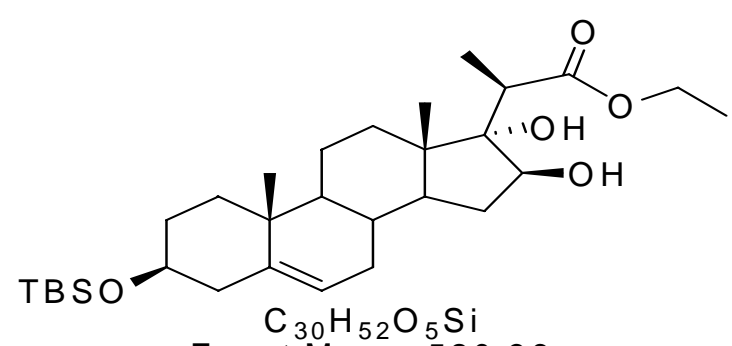

Exact Mass: 520.36

Mol. Wt.: 520.82

C, $69.18 ; \mathrm{H}, 10.06 ; 0,15.36 ; \mathrm{Si}, 5.39$

$\mathrm{CDCl}_{3}$

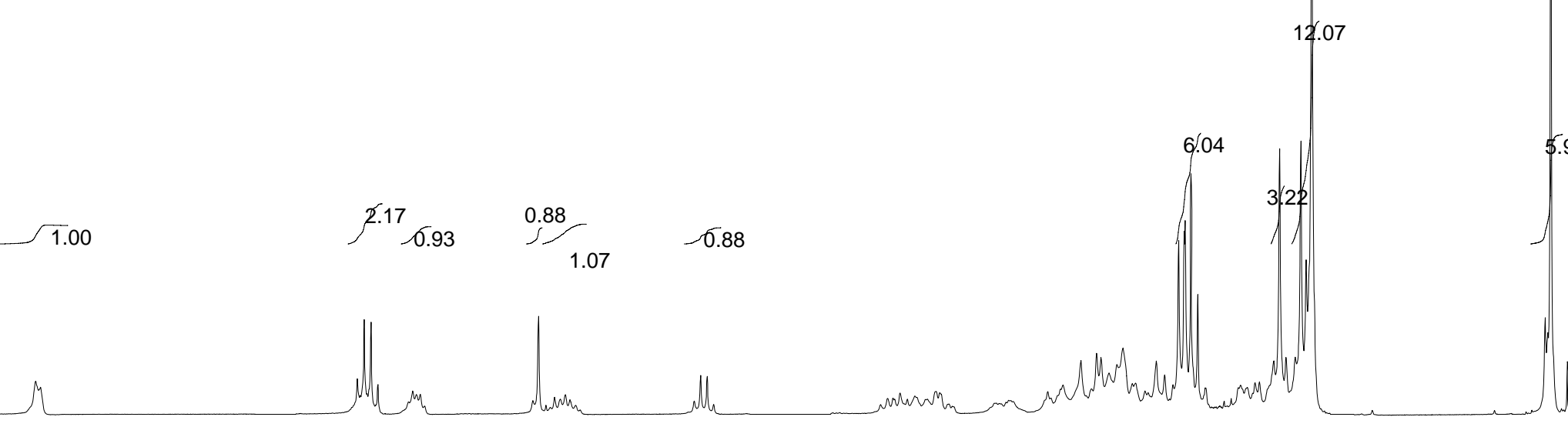

7 


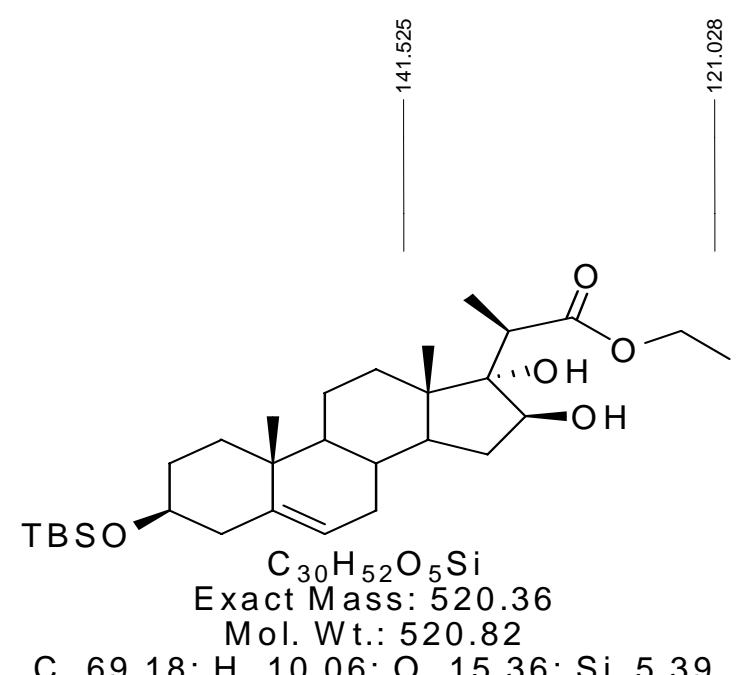

C, $69.18 ; \mathrm{H}, 10.06 ; \mathrm{O}, 15.36 ; \mathrm{Si}, 5.39$ $\mathrm{CDCl}_{3}$ 


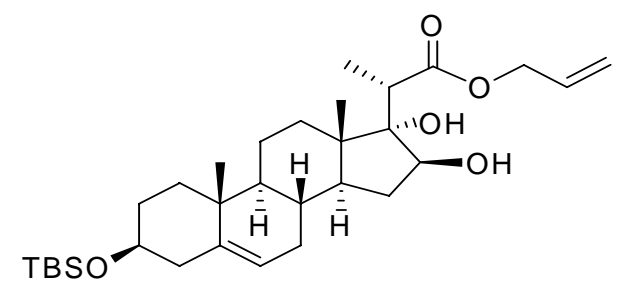

$\mathrm{C}_{31} \mathrm{H}_{52} \mathrm{O}_{5} \mathrm{Si}$

( Mt: 532.83

C, 69.88; H, 9.84; O, 15.01; Si, 5.27 


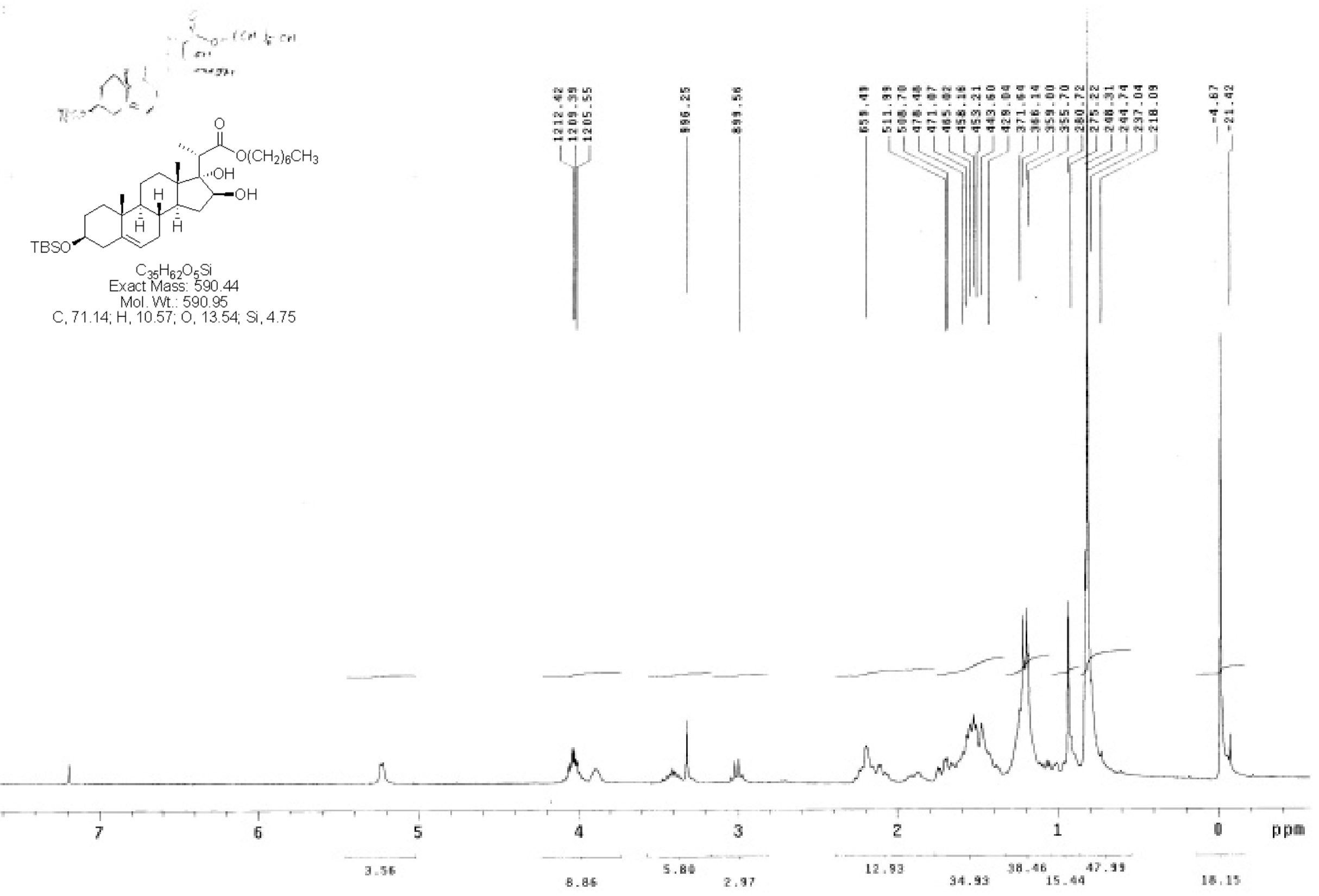

${ }^{1} \mathrm{H}$ NMR spectrum $(300 \mathrm{MHz})$ of $\mathbf{5 5}$ in $\mathrm{CDCl}_{3}$ 

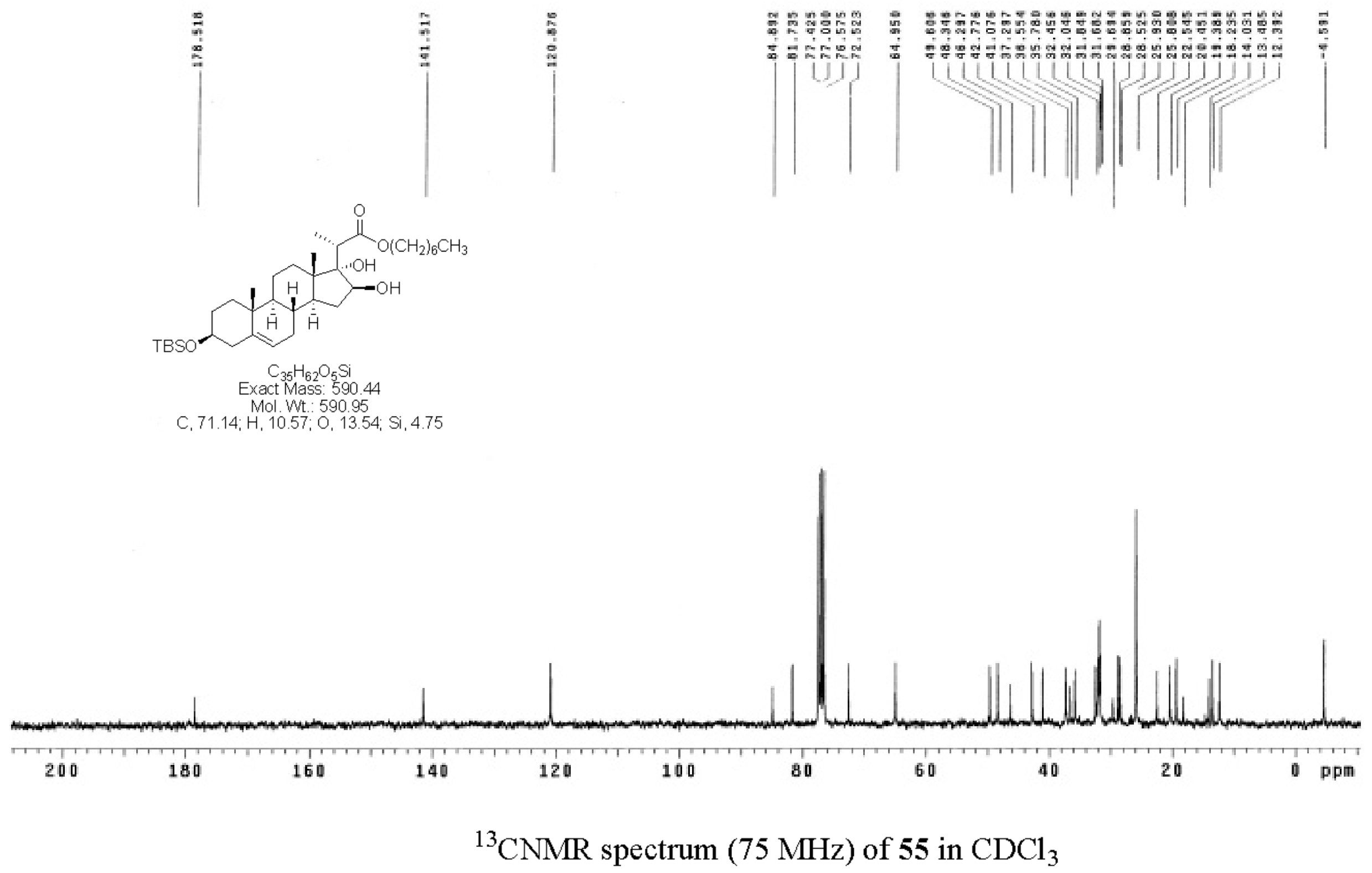

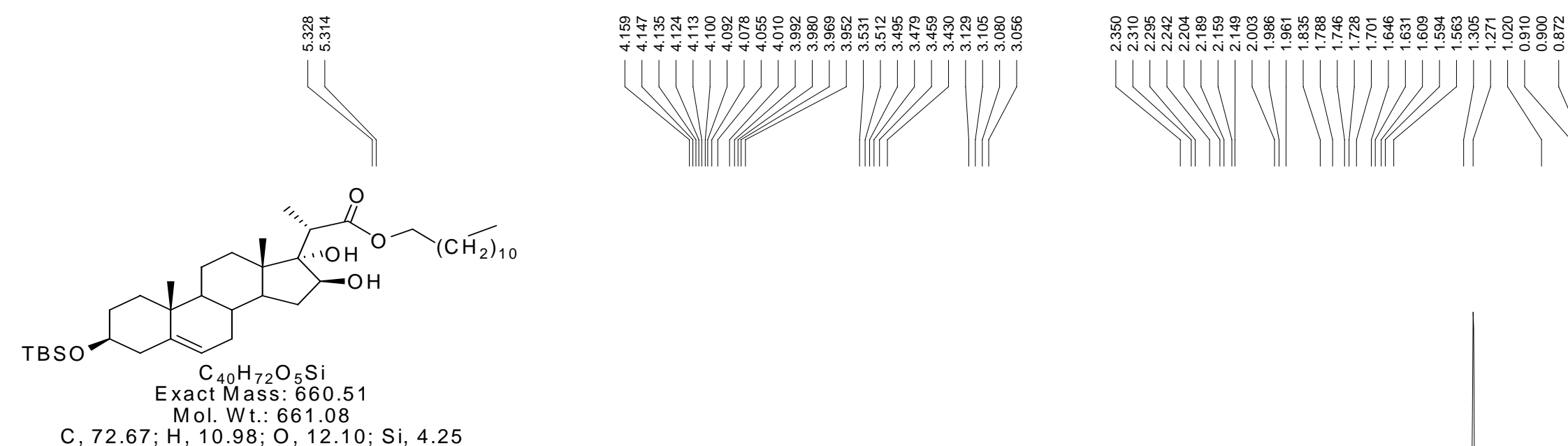

C, $72.67 ; \mathrm{H}, 10.98 ; 0,12.10 ; \mathrm{Si}, 4.25$

$$
\mathrm{CDCl}_{3}
$$

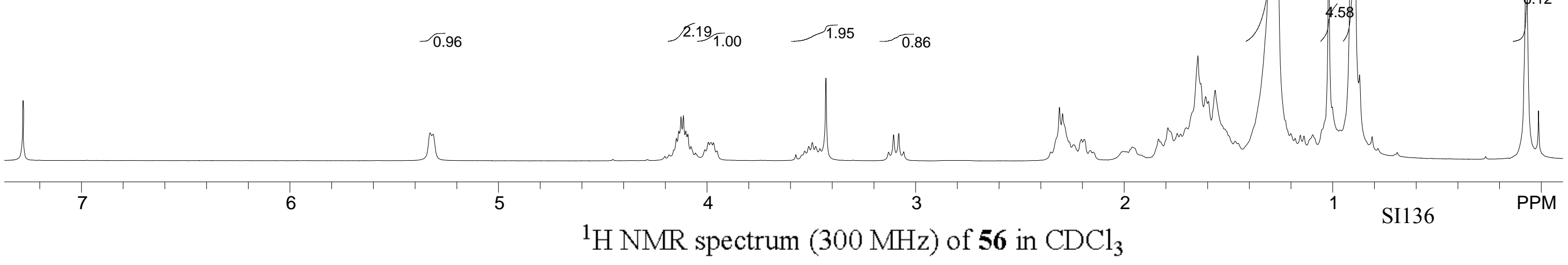




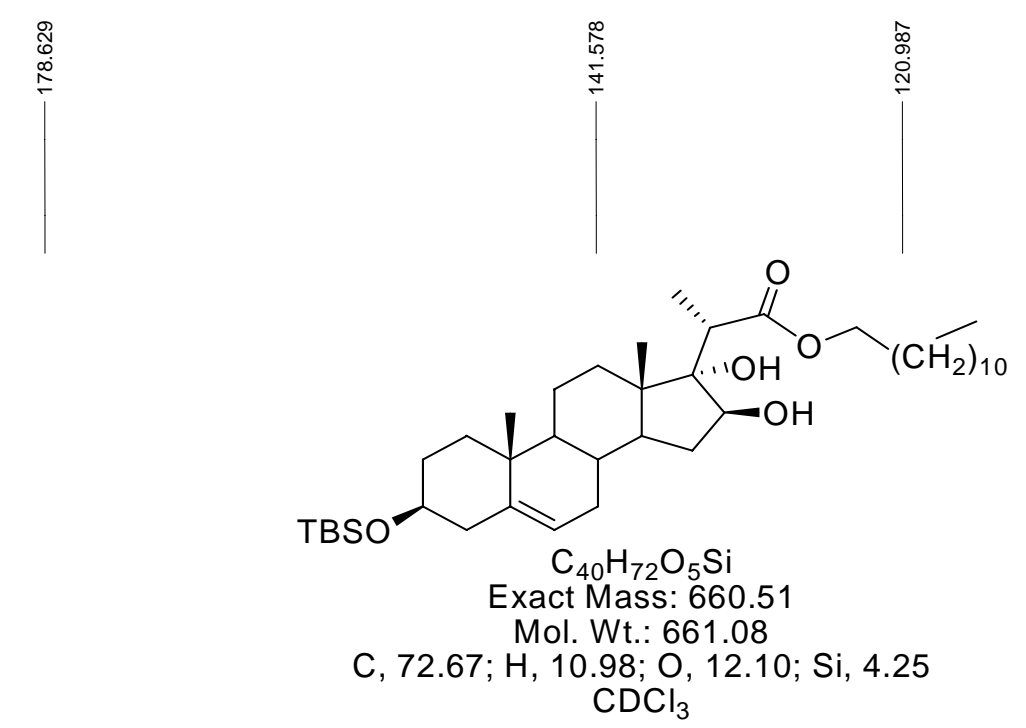

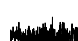




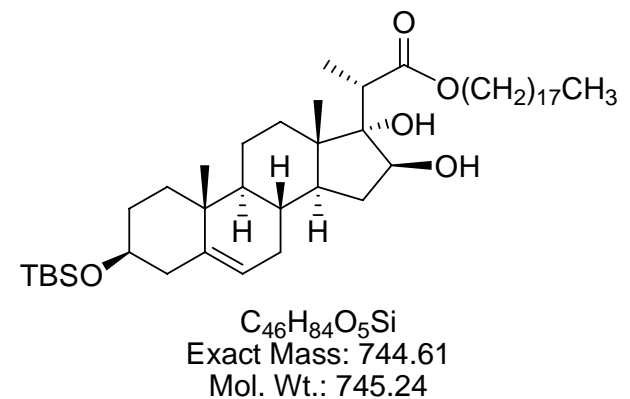

Mol. Wt.: 745.24
C, 74.14; H, 11.36; O, 10.73; Si, 3.77 


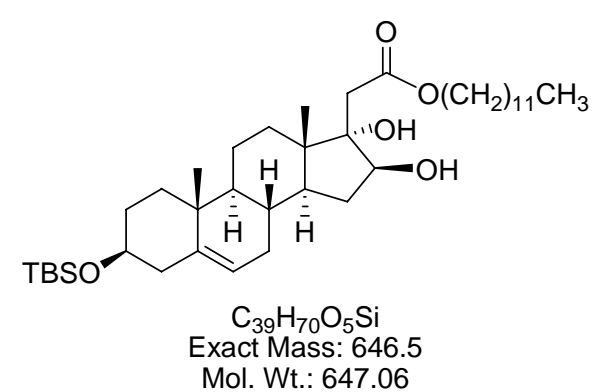

C, 72.39; Hol. Wt.: 647.06 ; 12.36 ; Si, 4.34 


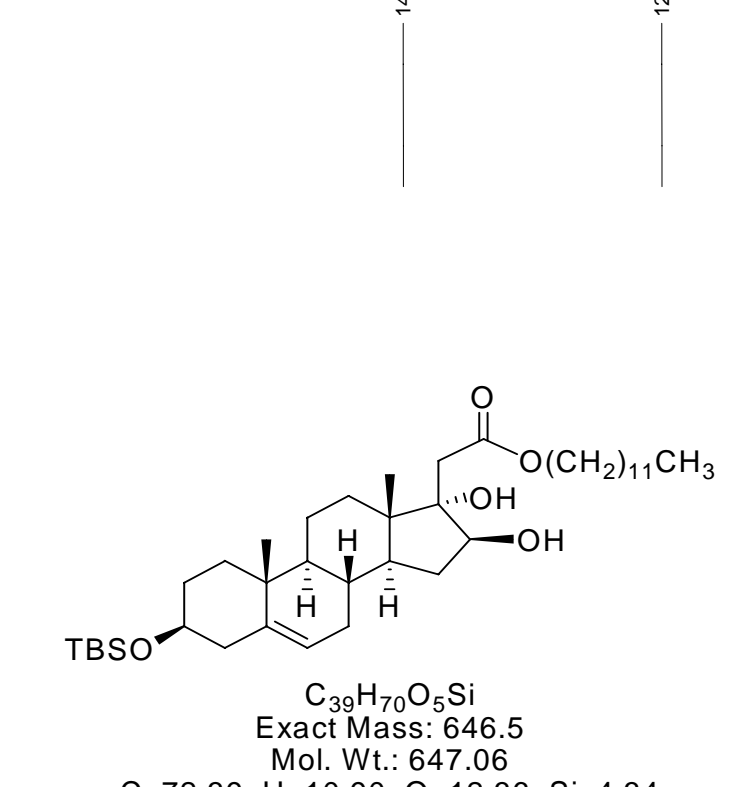

Mol. Wt.: 647.06

C, $72.39 ; \mathrm{H}, 10.90 ; \mathrm{O}, 12.36$; $\mathrm{Si}, 4.34$

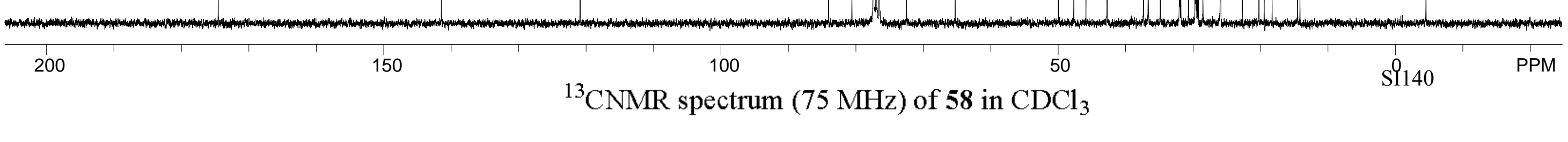



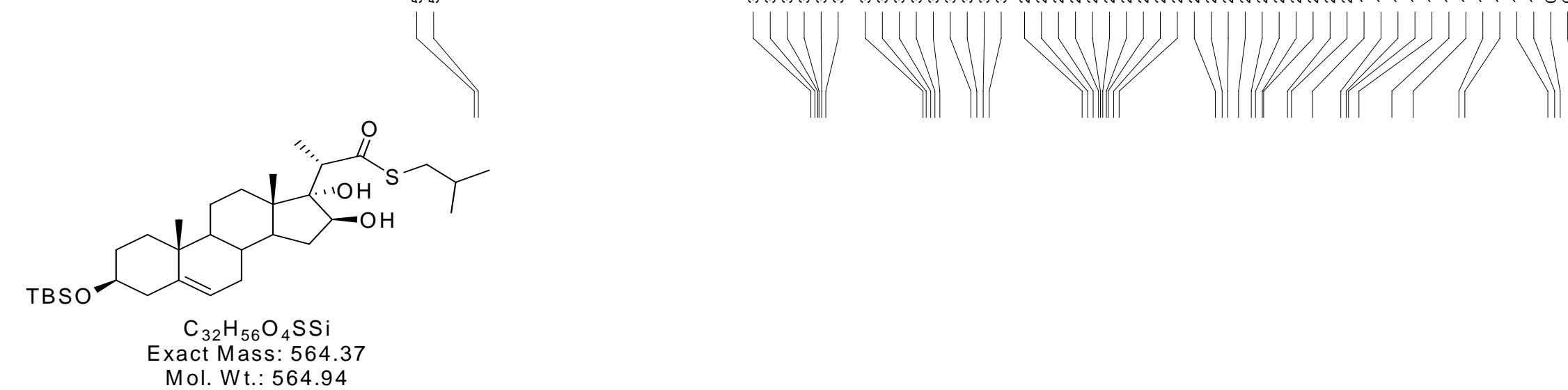

C, $68.03 ; \mathrm{H}, 9.99 ; 0,11.33 ; \mathrm{S}, 5.68 ; \mathrm{Si}, 4.97$ $\mathrm{CDCl}_{3}$

2

2

1
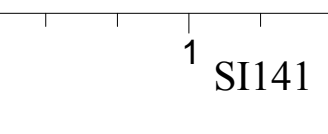

PPM 
TBSO

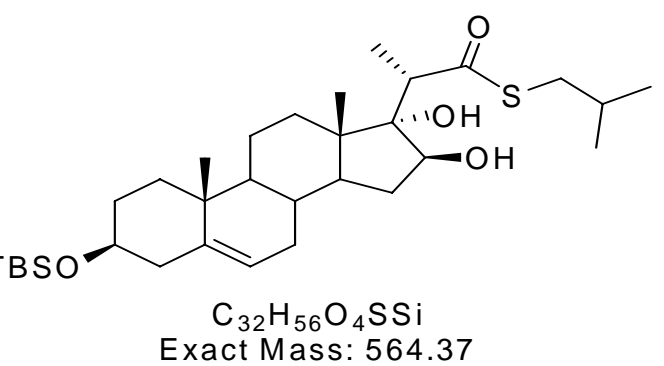

Exact Mass: 564.3

Mol. Wt.: 564.94

C, $68.03 ; \mathrm{H}, 9.99 ; \mathrm{O}, 11.33 ; \mathrm{S}, 5.68 ; \mathrm{Si}, 4.97$

$\mathrm{CDCl}_{3}$ 


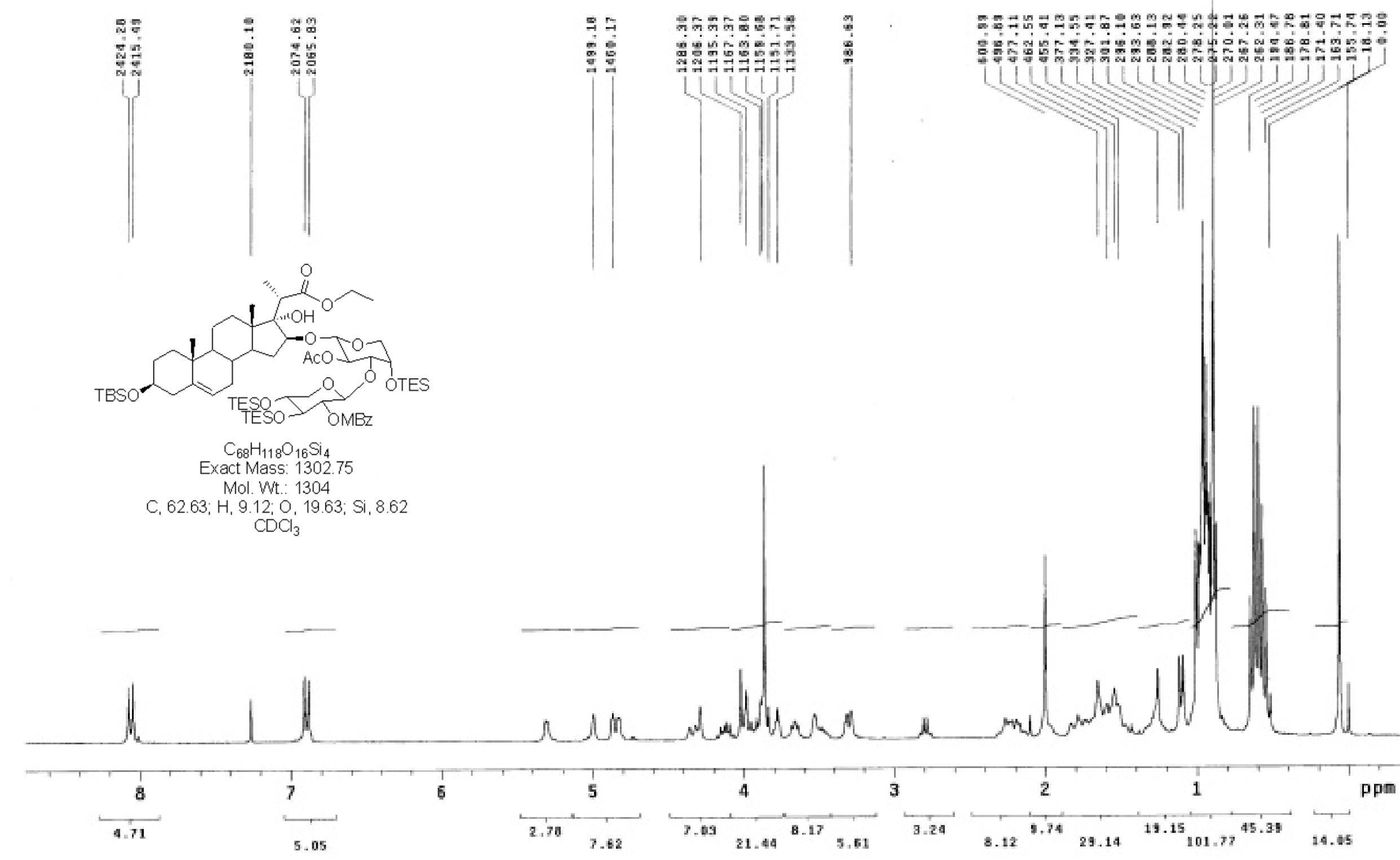

${ }^{1} \mathrm{H}$ NMR spectrum (300 MHz) of 60 in $\mathrm{CDCl}_{3}$ 


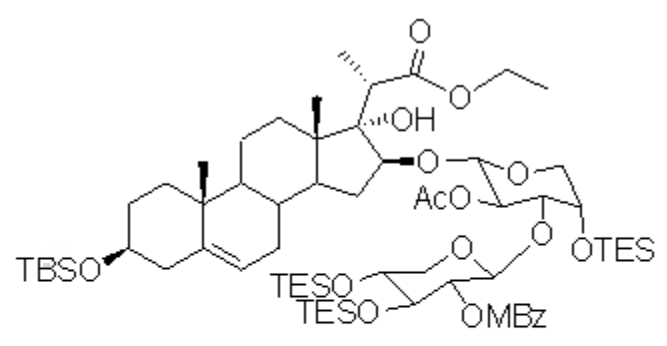

$\mathrm{C}_{68} \mathrm{H}_{118} \mathrm{O}_{16} \mathrm{Si}_{4}$

Exact Mass: 1302.75

C. $62.63 ; \mathrm{H}, 9.12 ; \mathrm{O}, 19.63 ; \mathrm{Si}, 8.62$

$$
\mathrm{CDCl}_{3}
$$

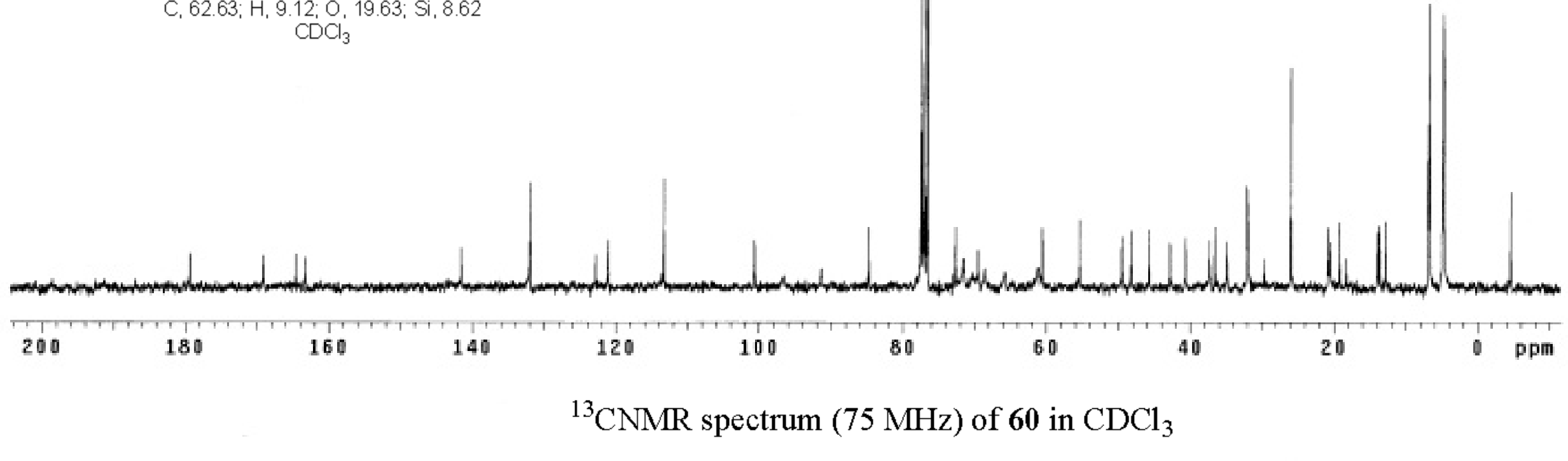




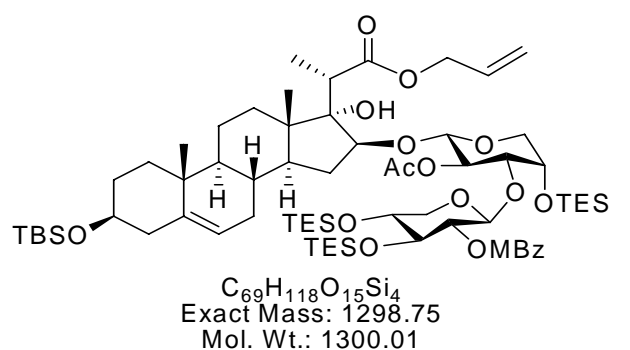

Mol. Wt.: 1300.01
C, $63.75 ; \mathrm{H}, 9.15 ; \mathrm{O}, 18.46 ; \mathrm{Si}, 8.64$

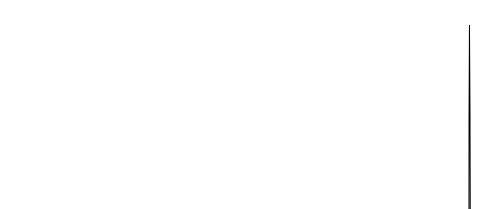




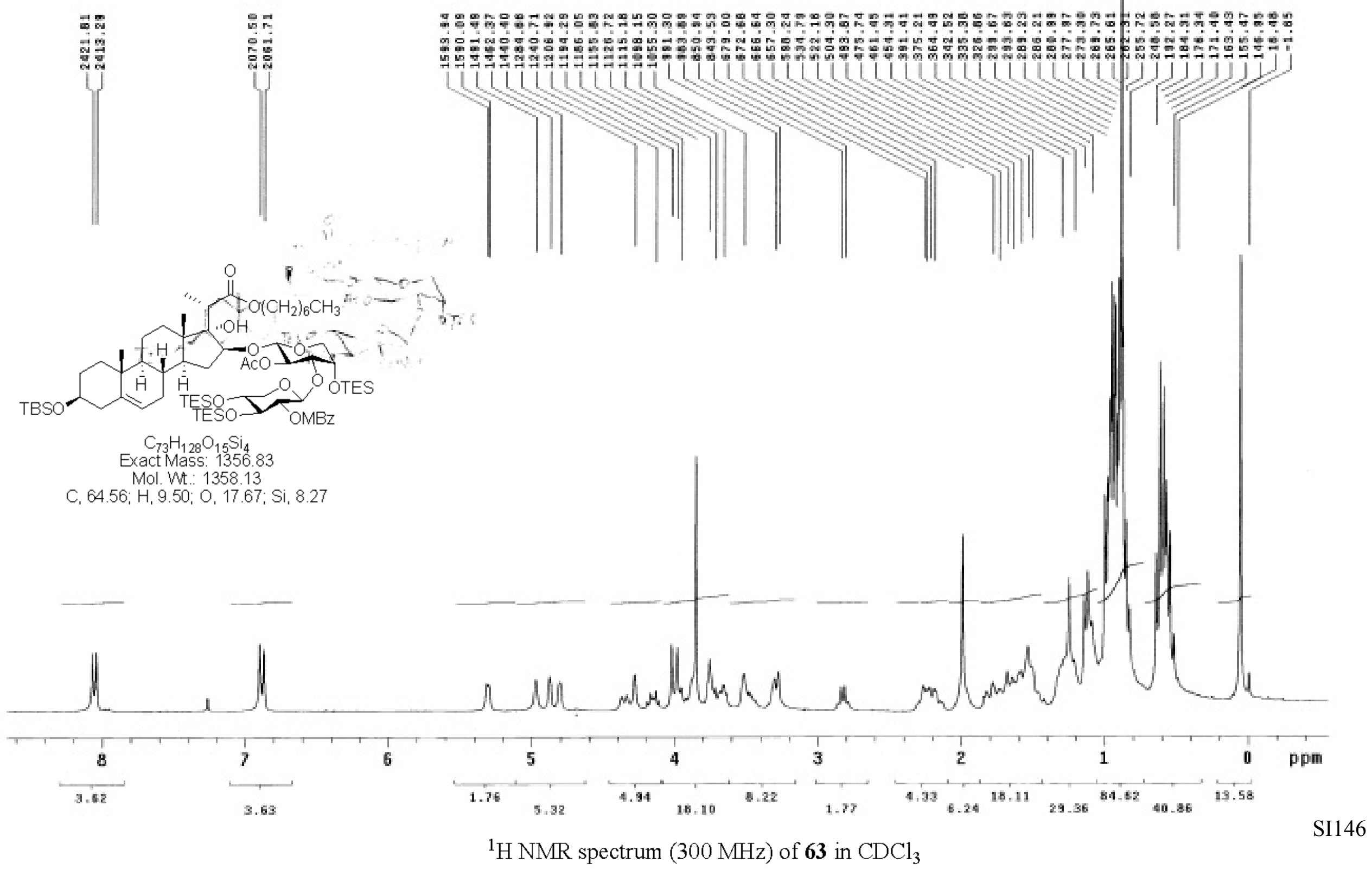




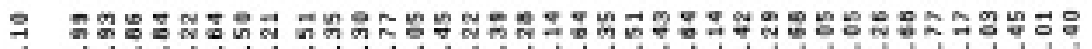

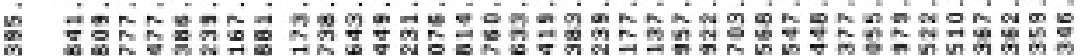
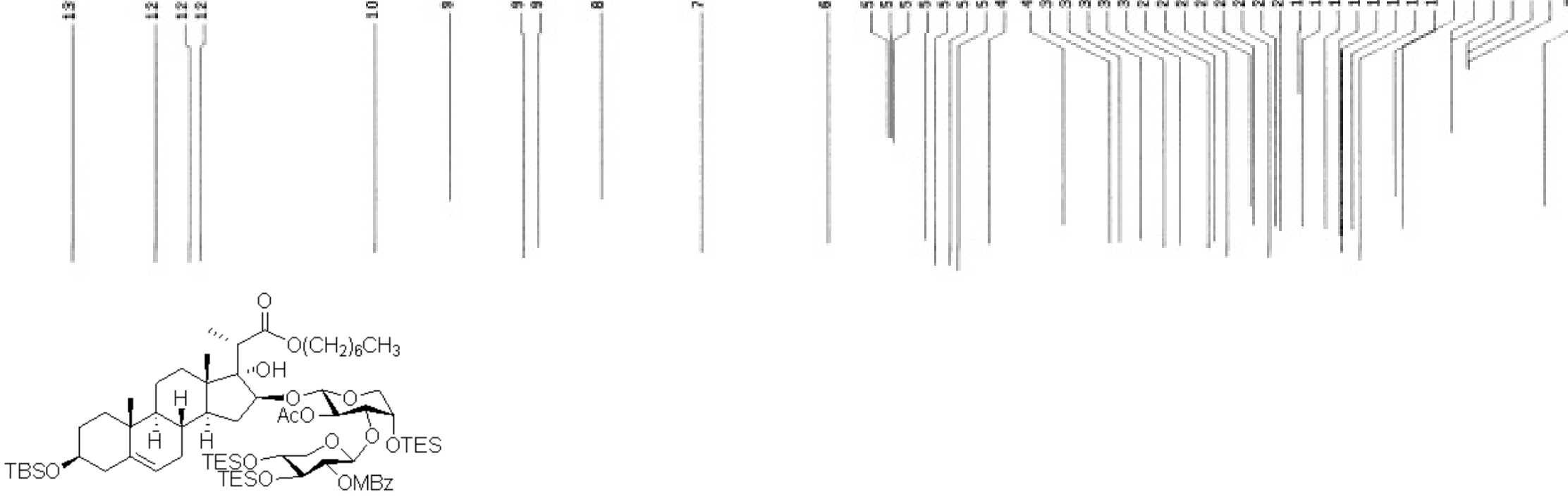

$\mathrm{C}_{73} \mathrm{H}_{128} \mathrm{O}_{15} \mathrm{Si}_{4}$

Mol Mt. 1358.13

C. $64.56 ; \mathrm{H}, 9.50 ; 0.17 .67 ;$ Si, 8.27

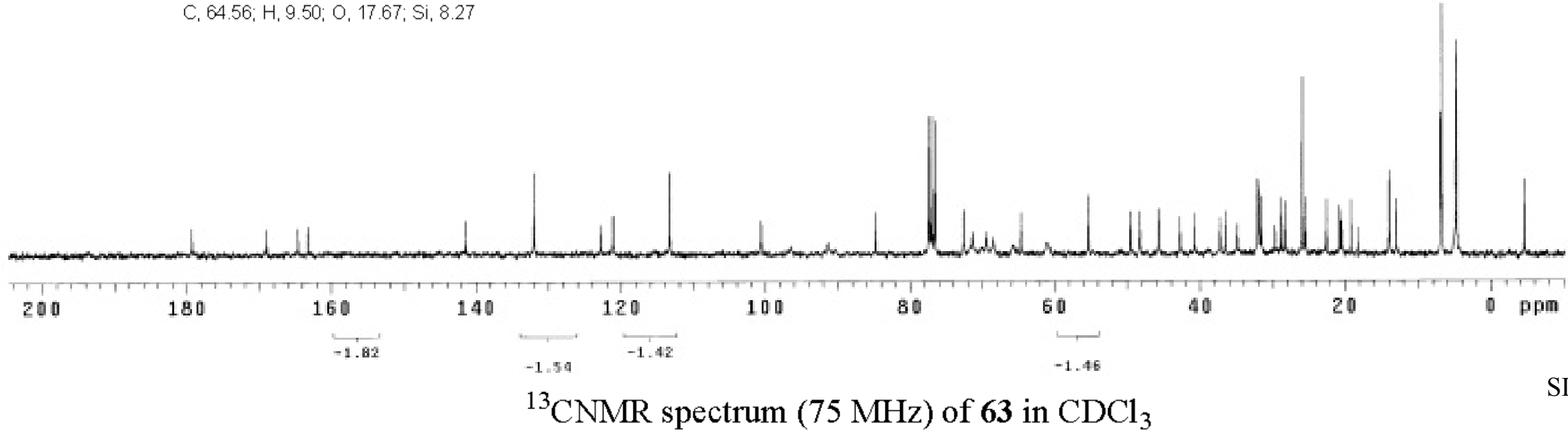



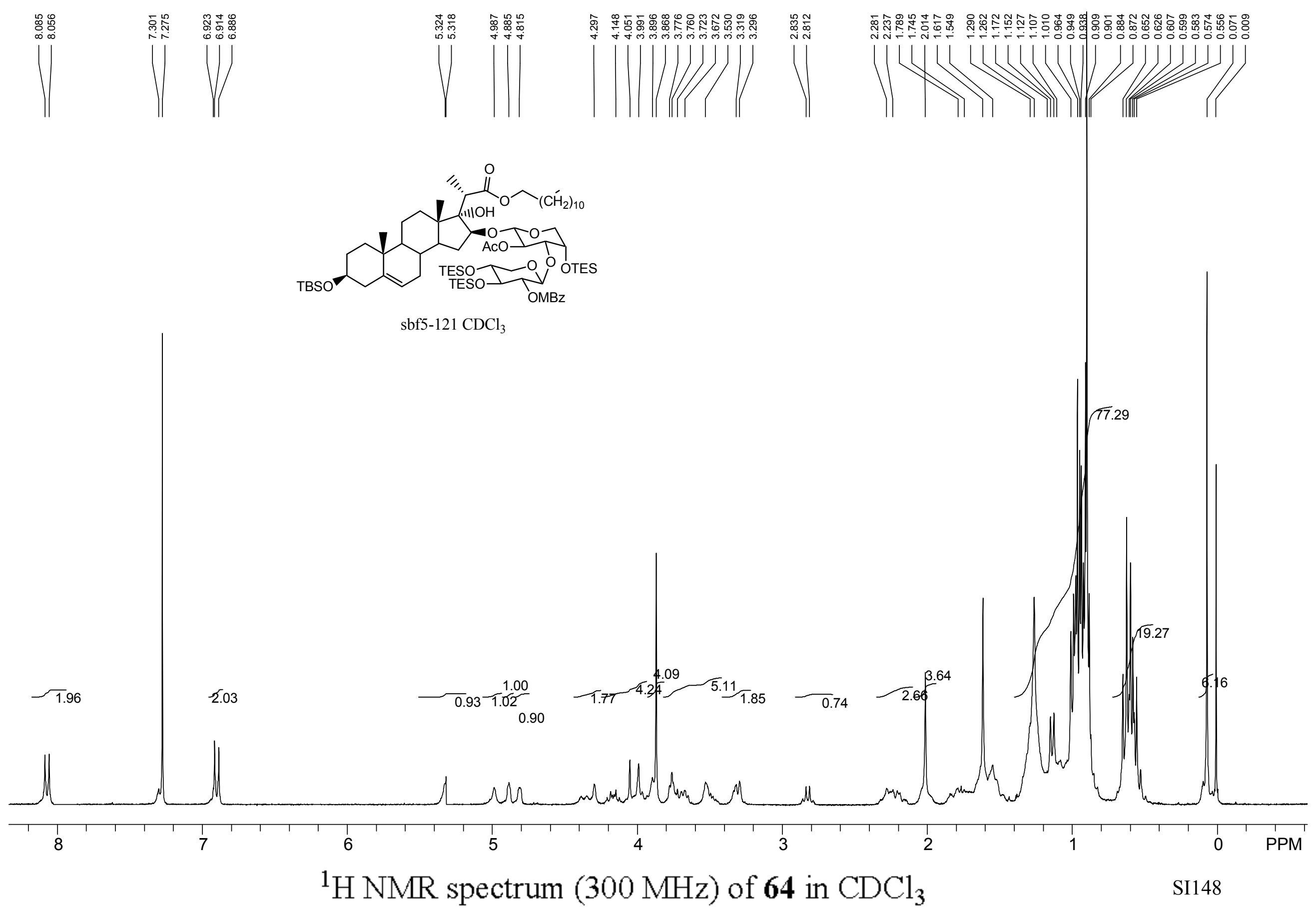

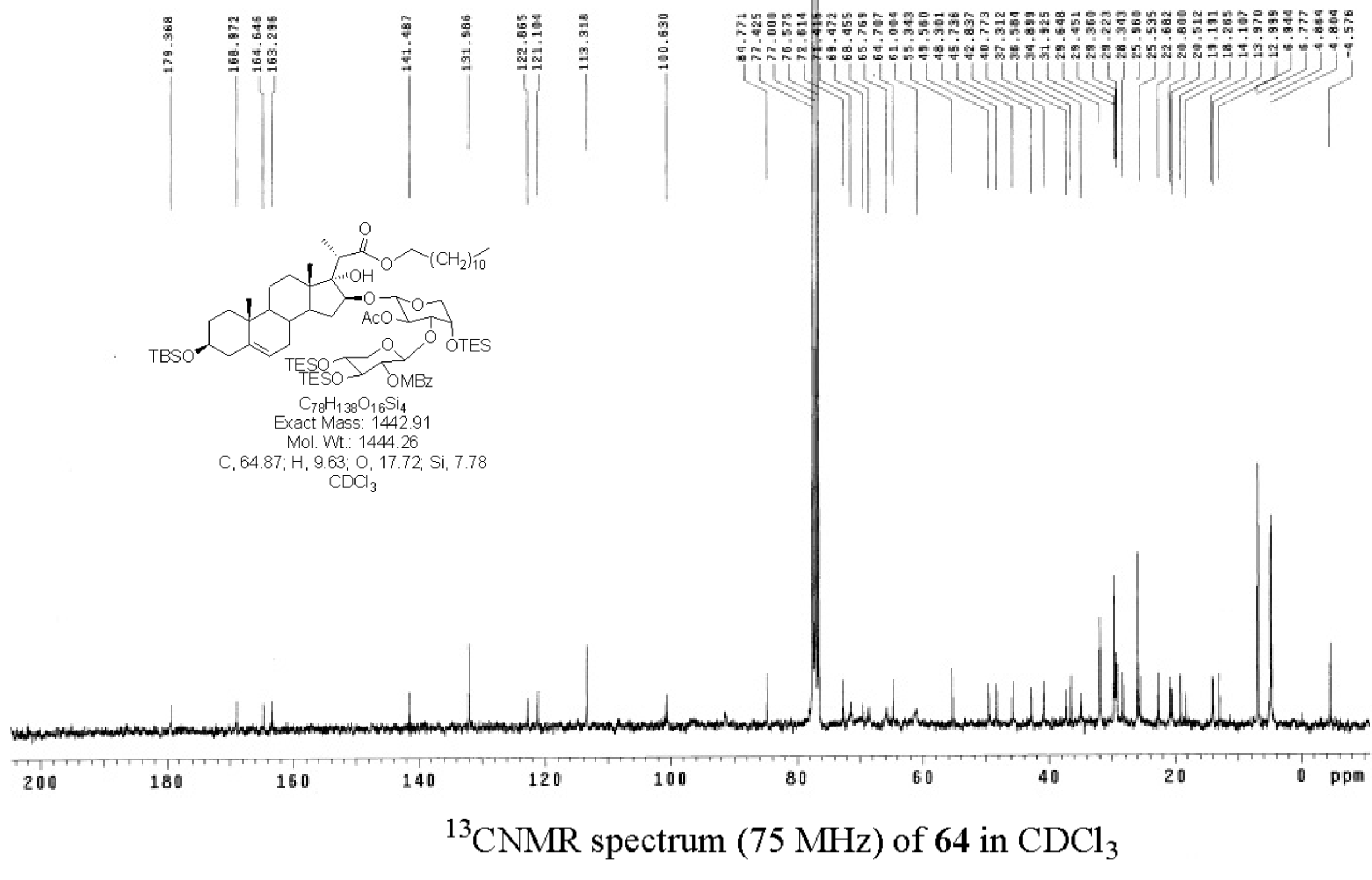


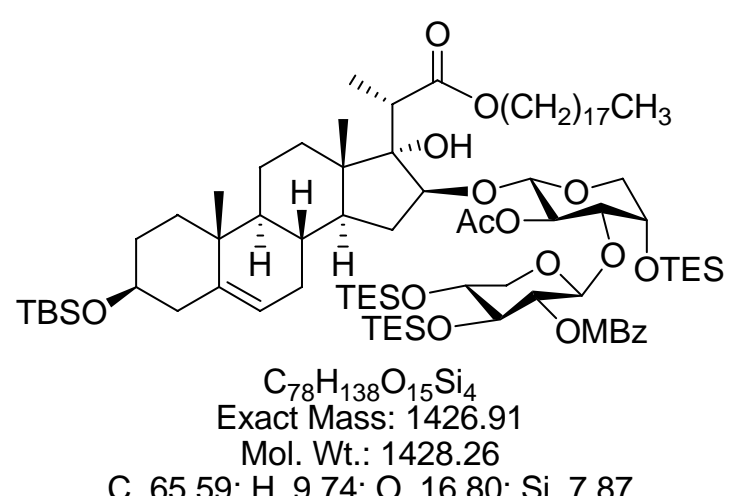




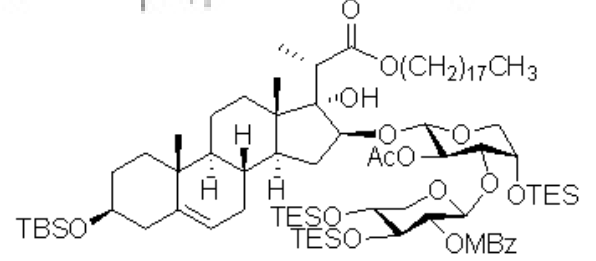
$\mathrm{C}_{84} \mathrm{H}_{150} \mathrm{O}_{15} \mathrm{Si}_{4}$ Mol Wh: 151242 C, $66.71 ; H, 10.00 ; 0,15.87 ;$ Si, 7.43

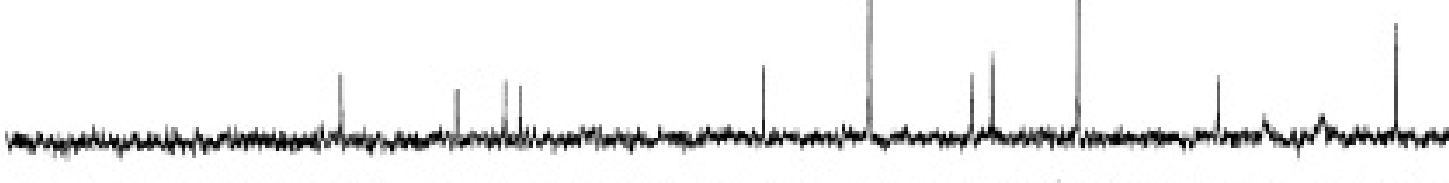

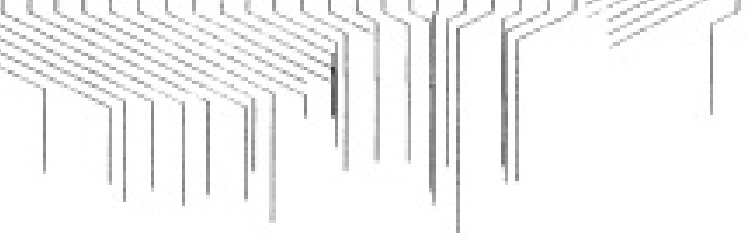

${ }^{13} \mathrm{CNMR}$ spectrum $(75 \mathrm{MHz})$ of 65 in $\mathrm{CDCl}_{3}$ 


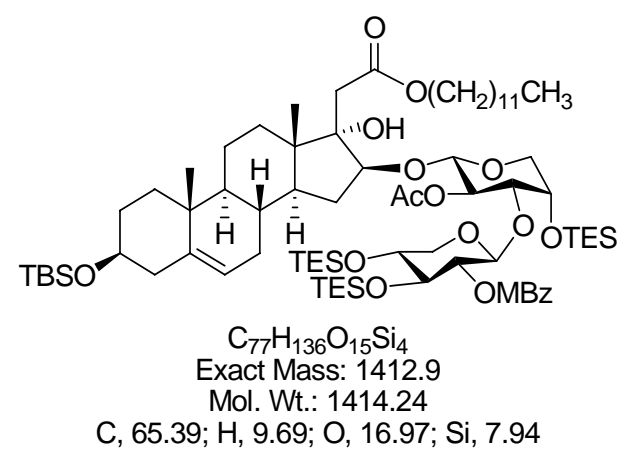

2.12

2.13

1.00

2.01 4.29 1.072 .02

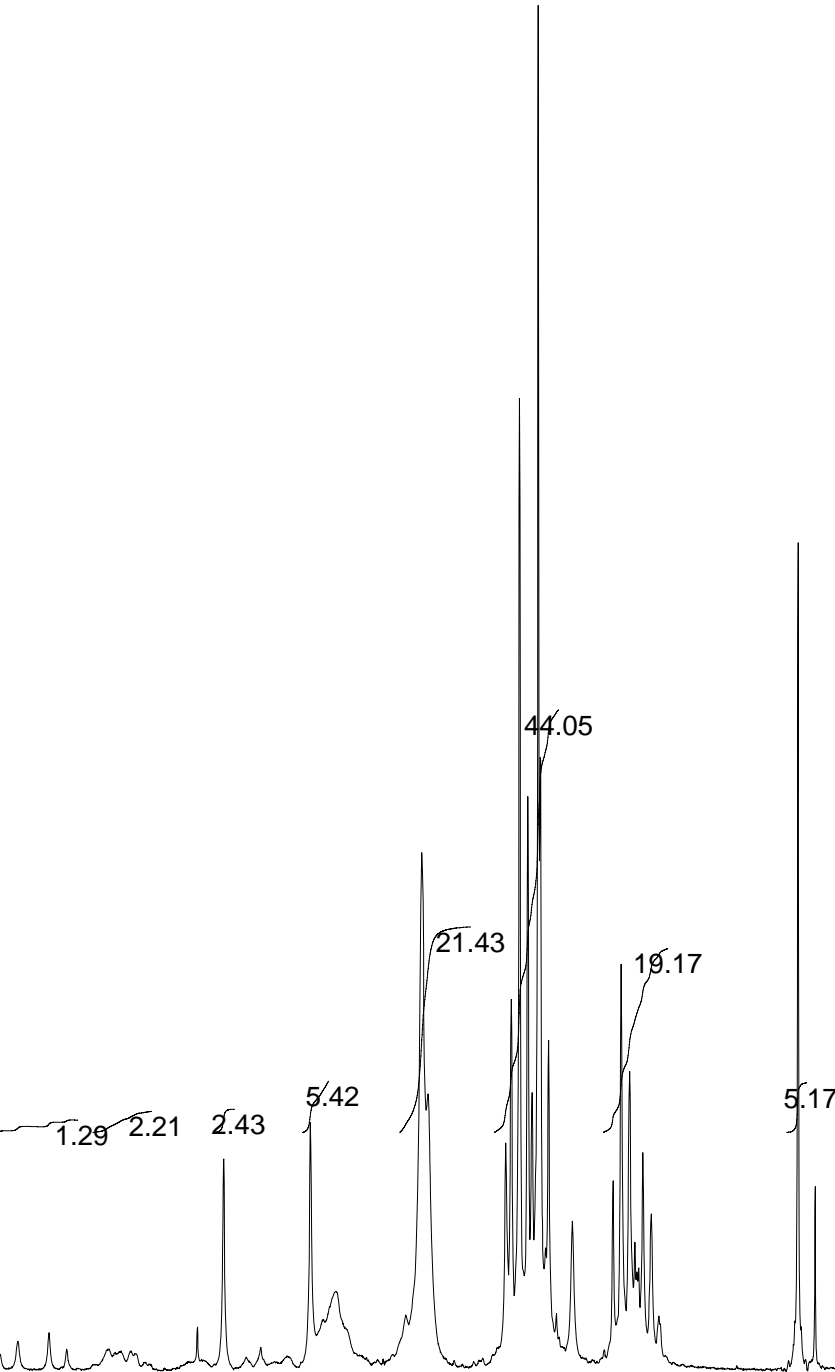




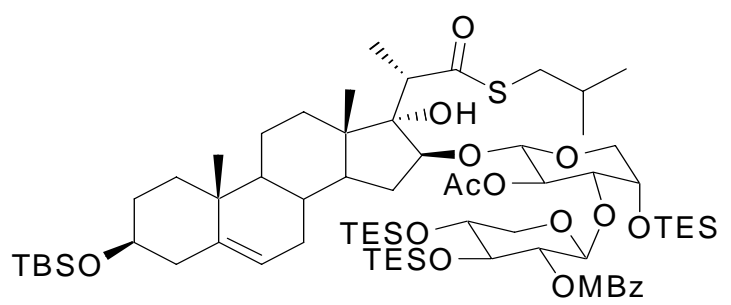

$\mathrm{C}_{70} \mathrm{H}_{122} \mathrm{O}_{15} \mathrm{SSi}_{4}$

Exact Mass: 1346.7582

Mol. Wt.: 1348.1167

C, 62.36; H, 9.12; O, 17.80; S, 2.38; Si, 8.33
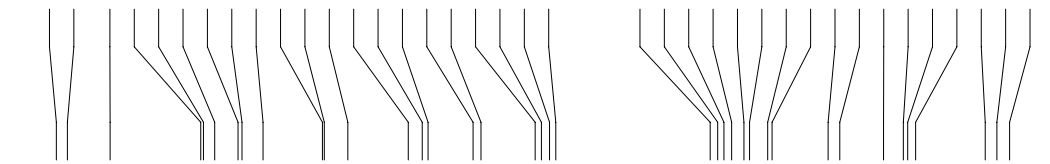


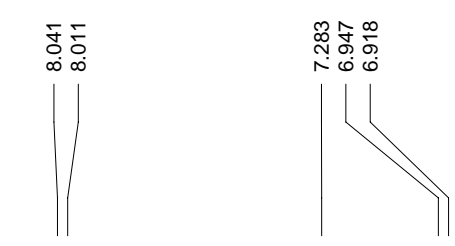

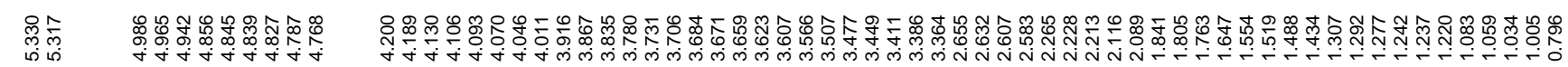
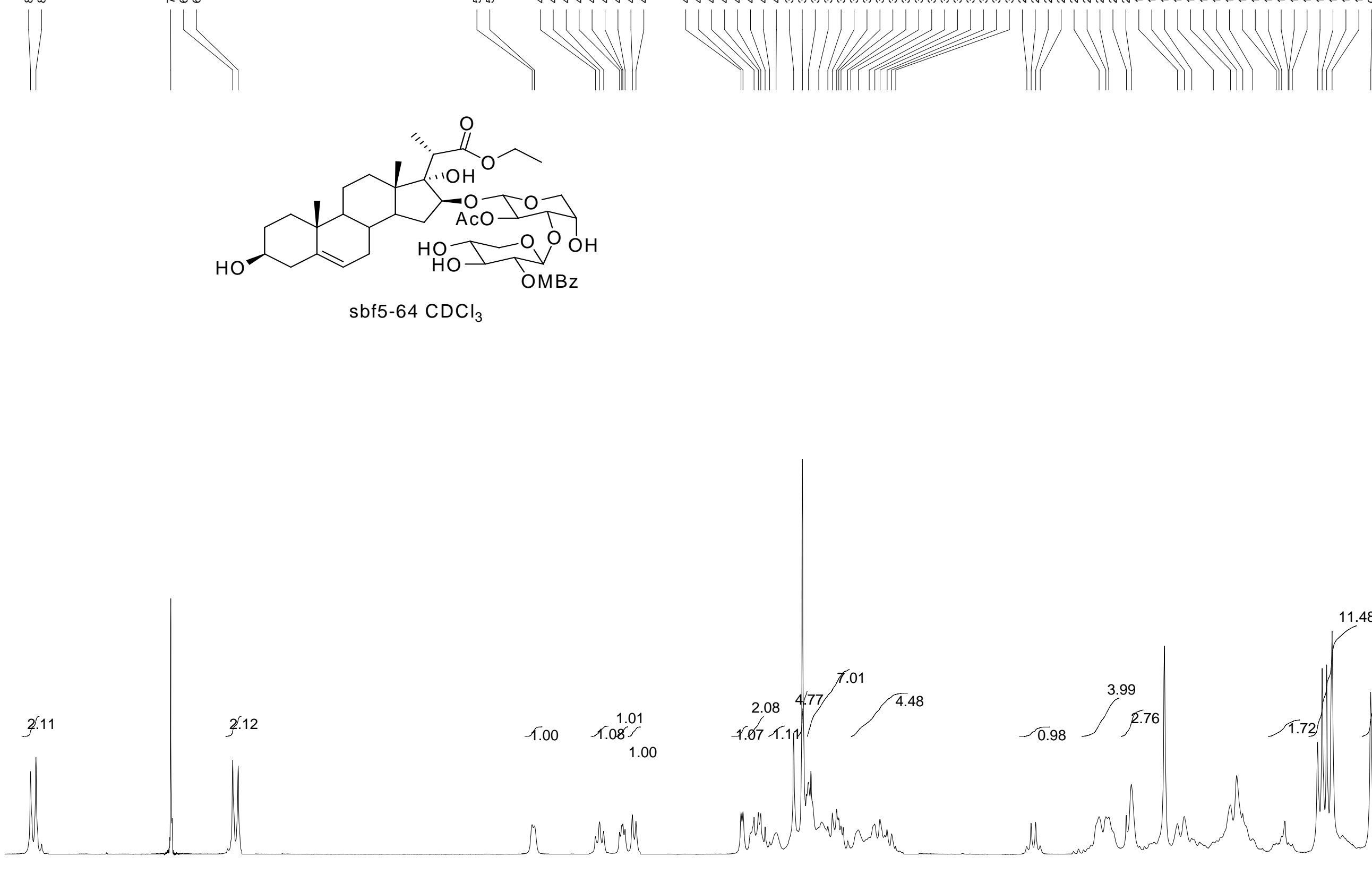

2.12
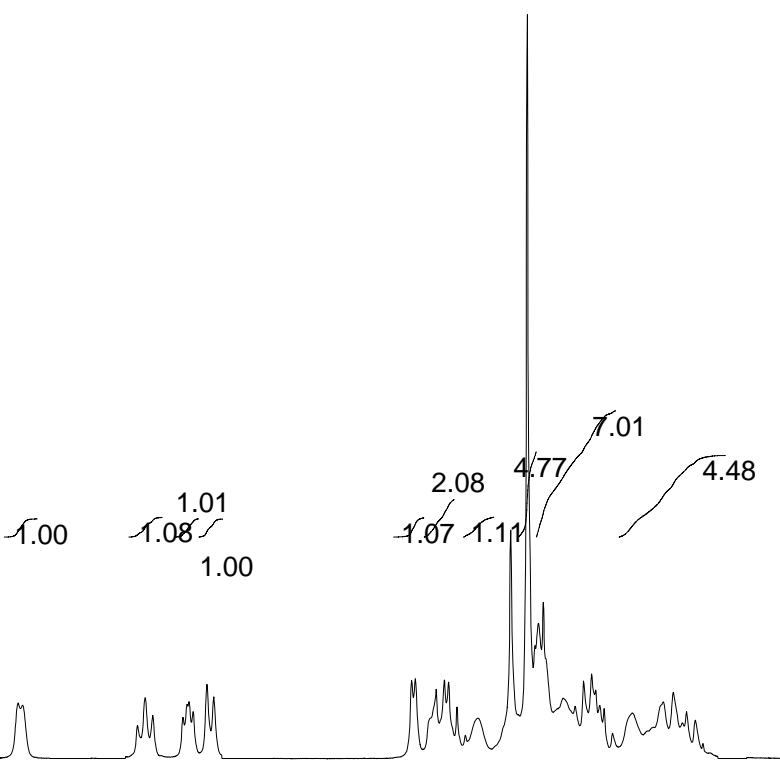

6

5

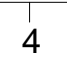

${ }^{1} \mathrm{H}$ NMR spectrum $(300 \mathrm{MHz})$ of $\mathbf{6 8}$ in $\mathrm{CDCl}_{3}$

2

SI154

PPM 

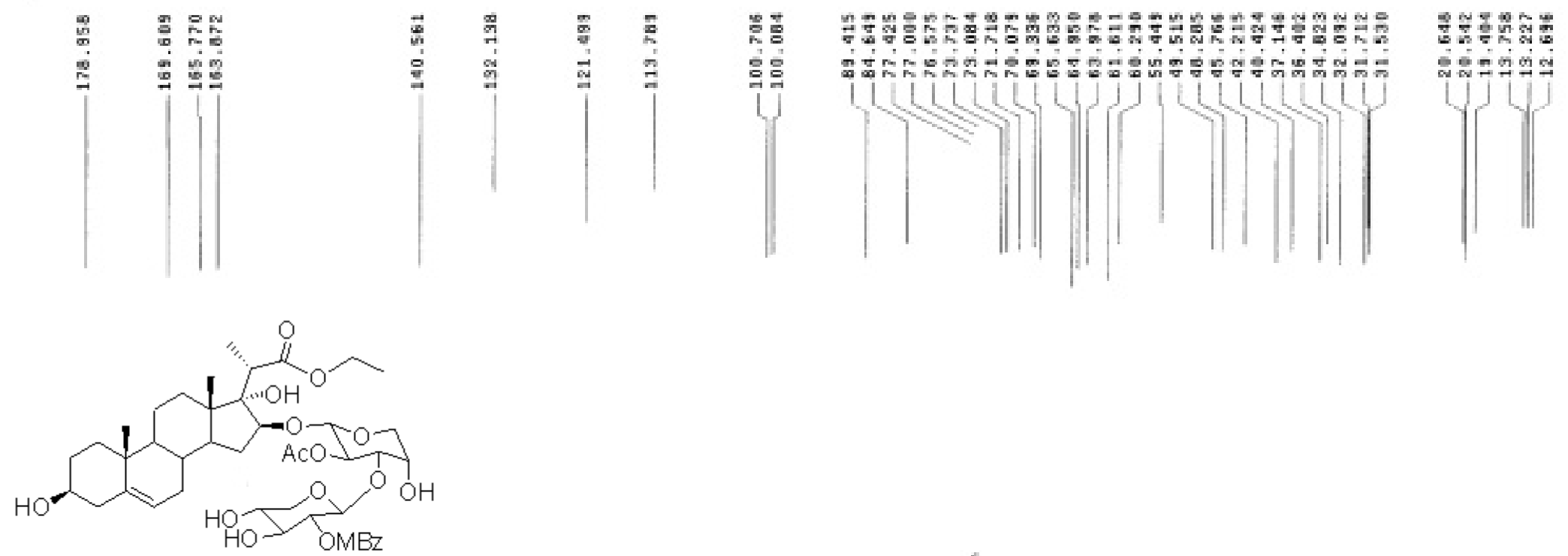

$\mathrm{C}_{44} \mathrm{H}_{62} \mathrm{O}_{16}$

Exact Mass: 8464

Mol. Wt.: 846.95

C. $62.40 ; H, 7.38 ; 0,30.22$

$$
\mathrm{CDCl}_{3}
$$

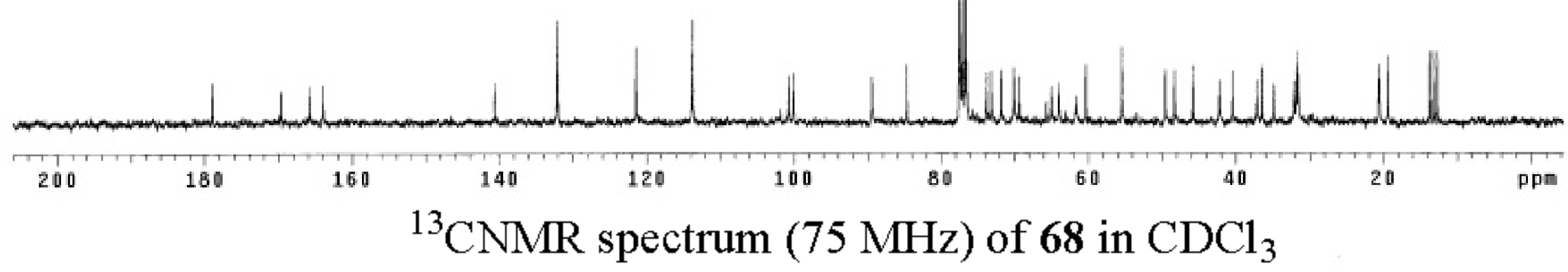




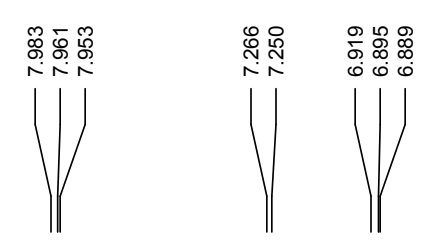

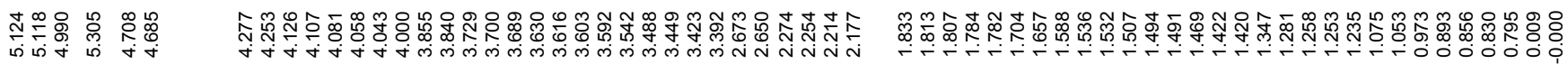
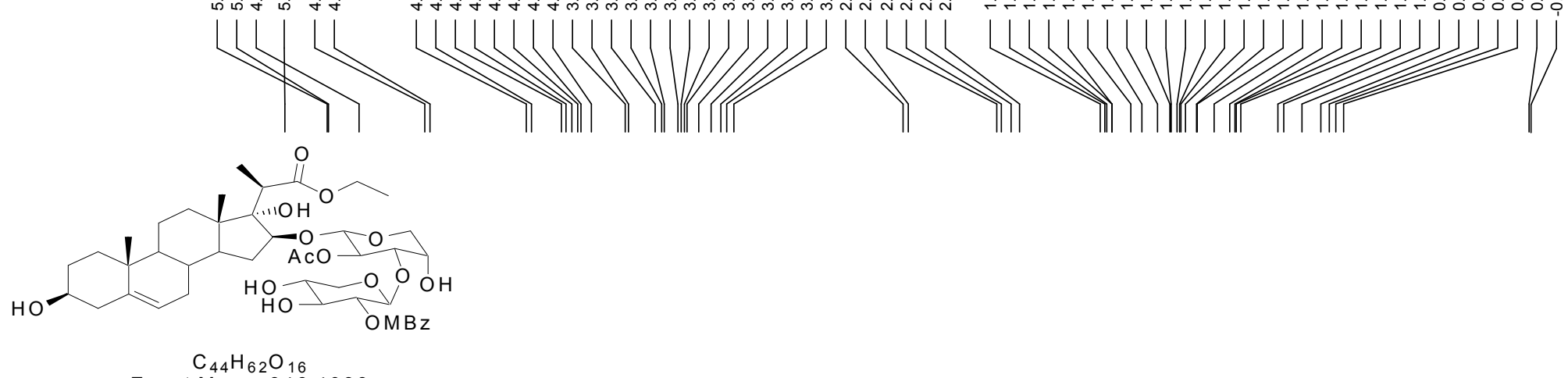

$\mathrm{Mol} \mathrm{Wt} \cdot 846.9535$

C, $62.40 ; \mathrm{H}, 7.38 ; \mathrm{O}, 30.22$

SBF5-125 03.12.30 $\mathrm{CDCl}_{3}$

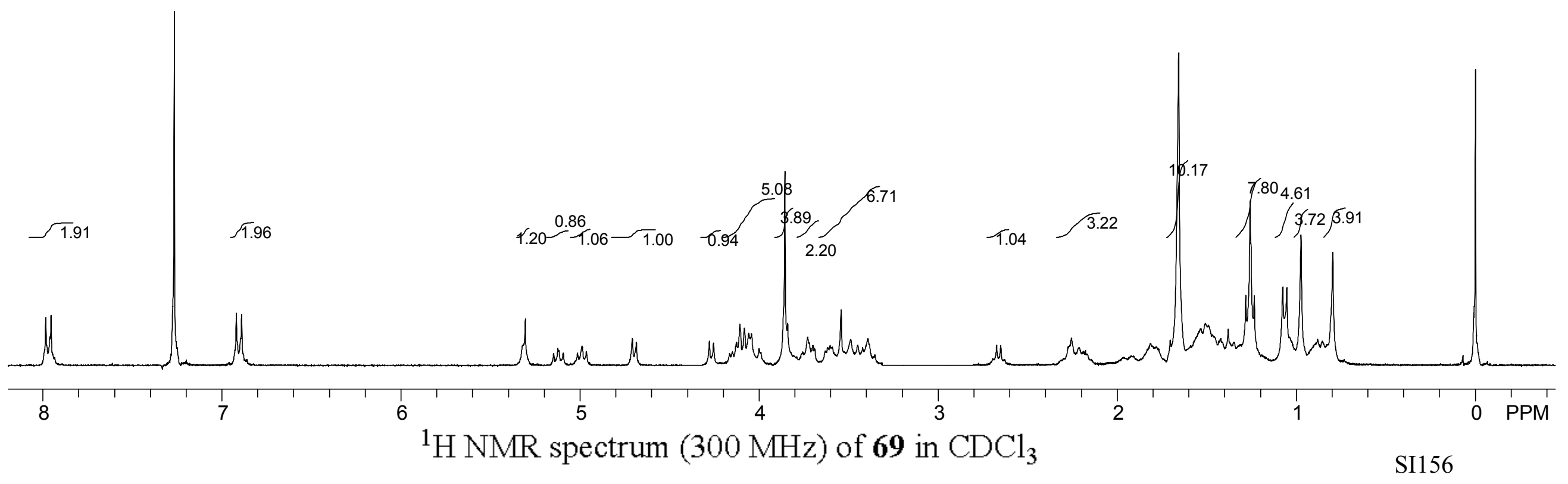




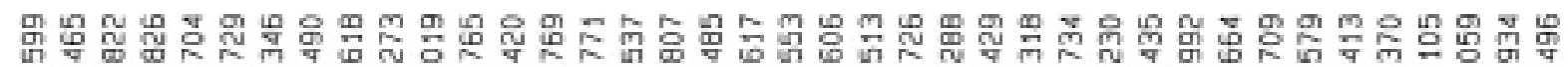
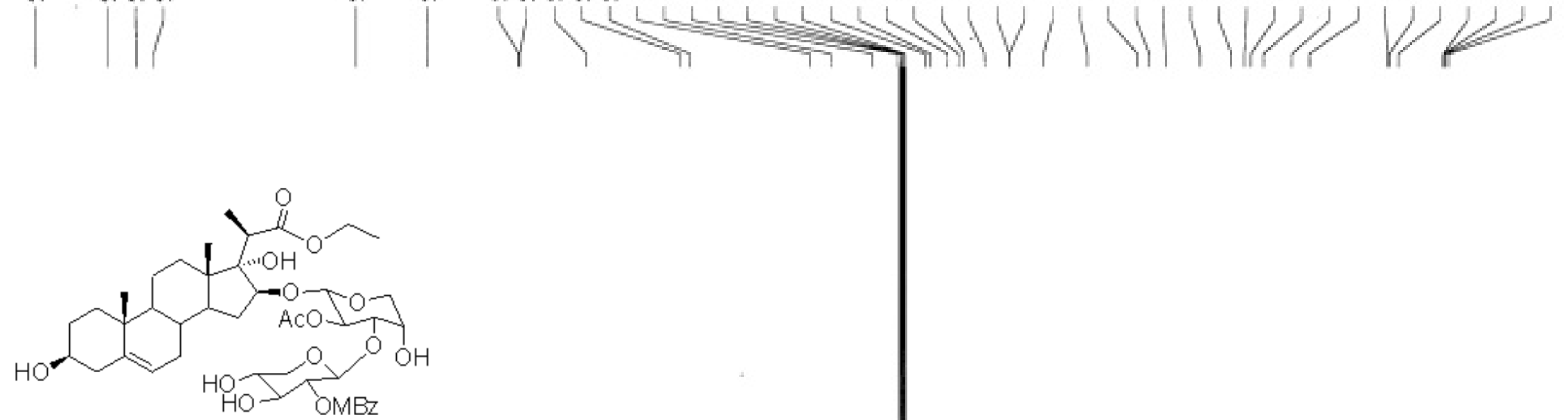

$\mathrm{C}_{44} \mathrm{H}_{62} \mathrm{O}_{16}$

Exact Mass: 846.4

Mol. Wt:: 846.95

C. $62.40 ; \mathrm{H}, 7.38 ; 0,30.22$

$\mathrm{CDCl}_{3}$

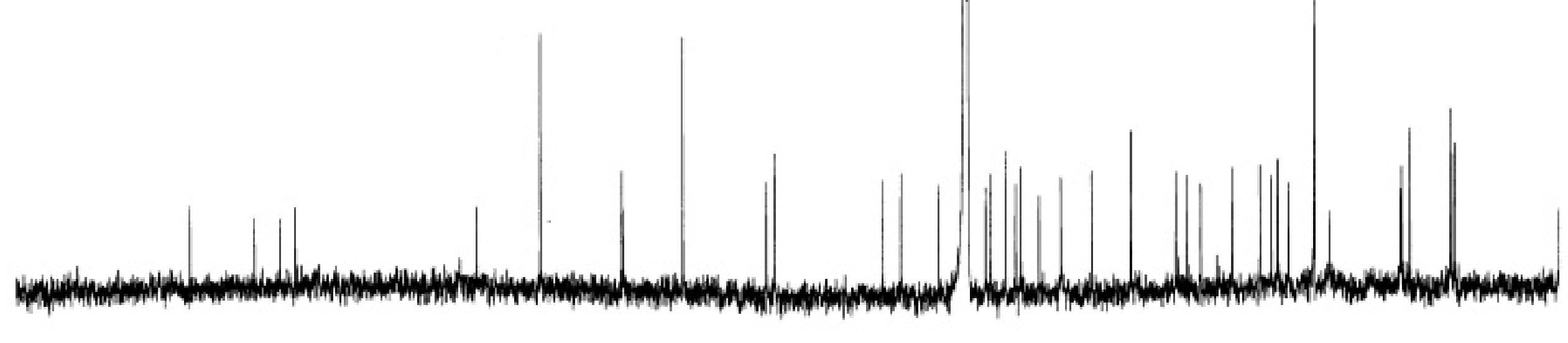




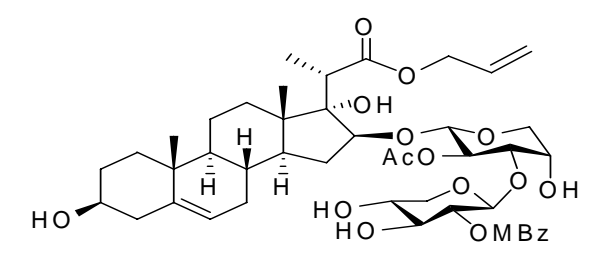

$\mathrm{C}_{45} \mathrm{H}_{62} \mathrm{O}_{15}$
Exact Mass: 842.41

Mol. Wt:: 842.96

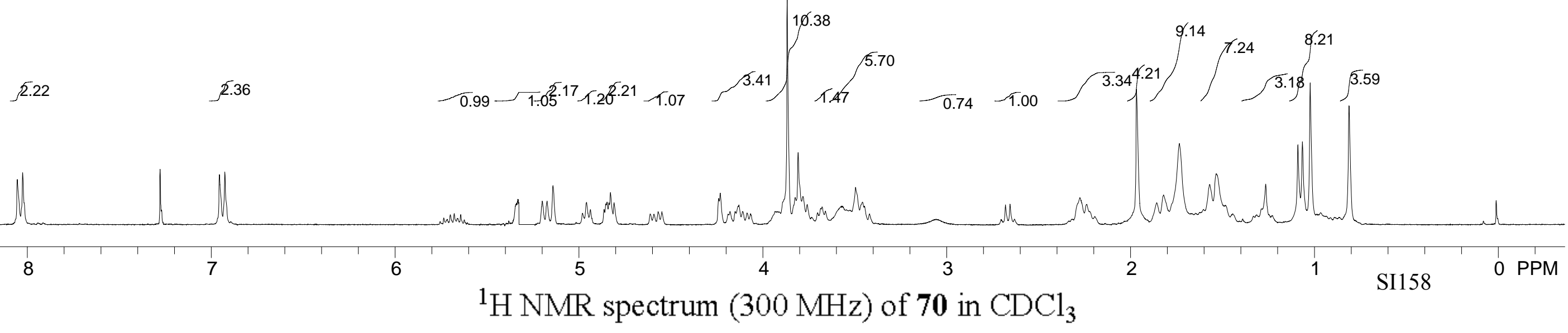




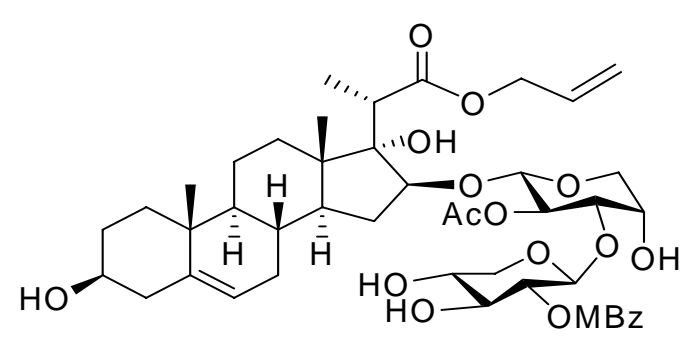

$\mathrm{C}_{45} \mathrm{H}_{62} \mathrm{O}$

Exact Mass: 842.41

Mol. Wt.: 842.96

C, $64.12 ; \mathrm{H}, 7.41 ; \mathrm{O}, 28.47$

M. 


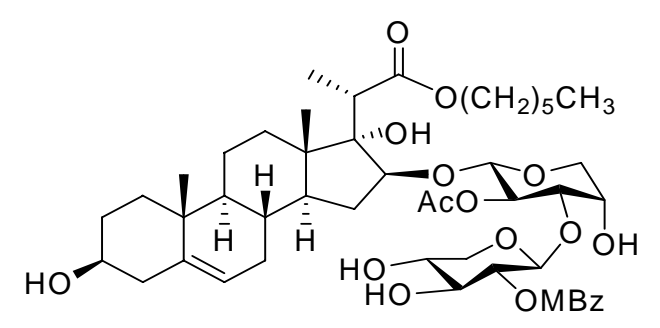

$\mathrm{C}_{48} \mathrm{H}_{70} \mathrm{O}$

Exact Mass: 886.47

Mol. Wt.: 887.06

C, 64.99; H, 7.95; O, 27.05

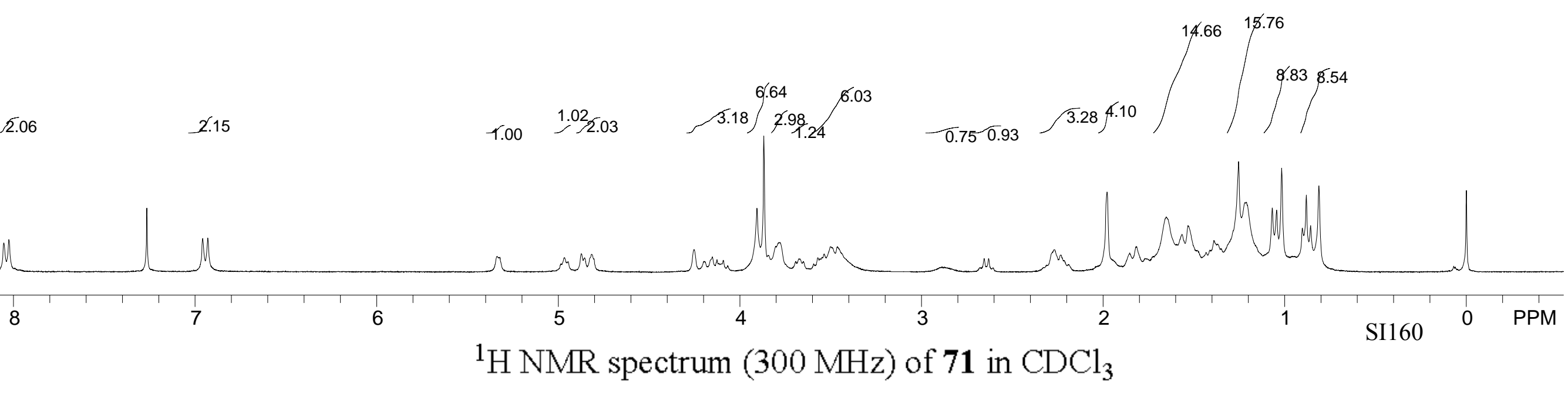



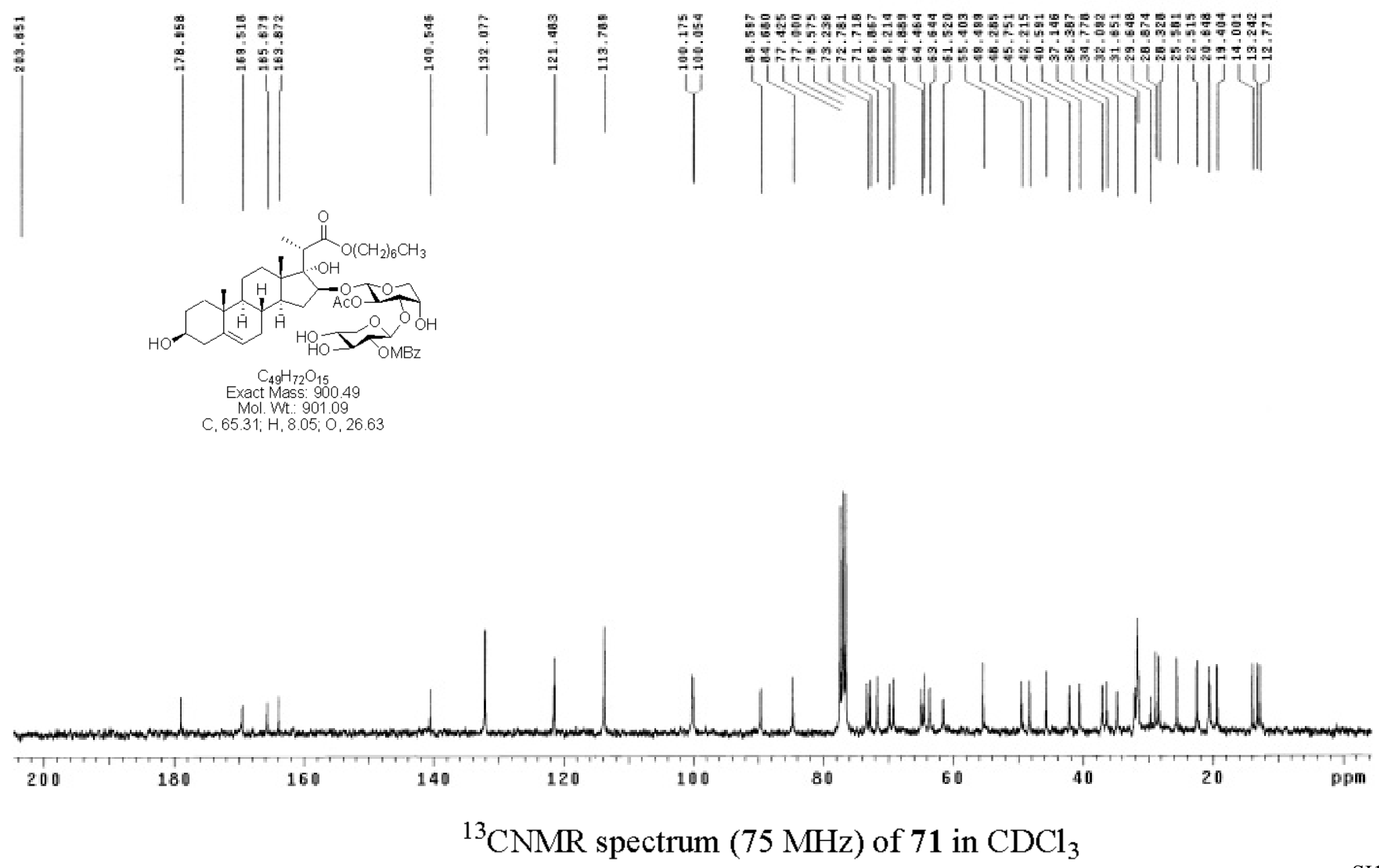


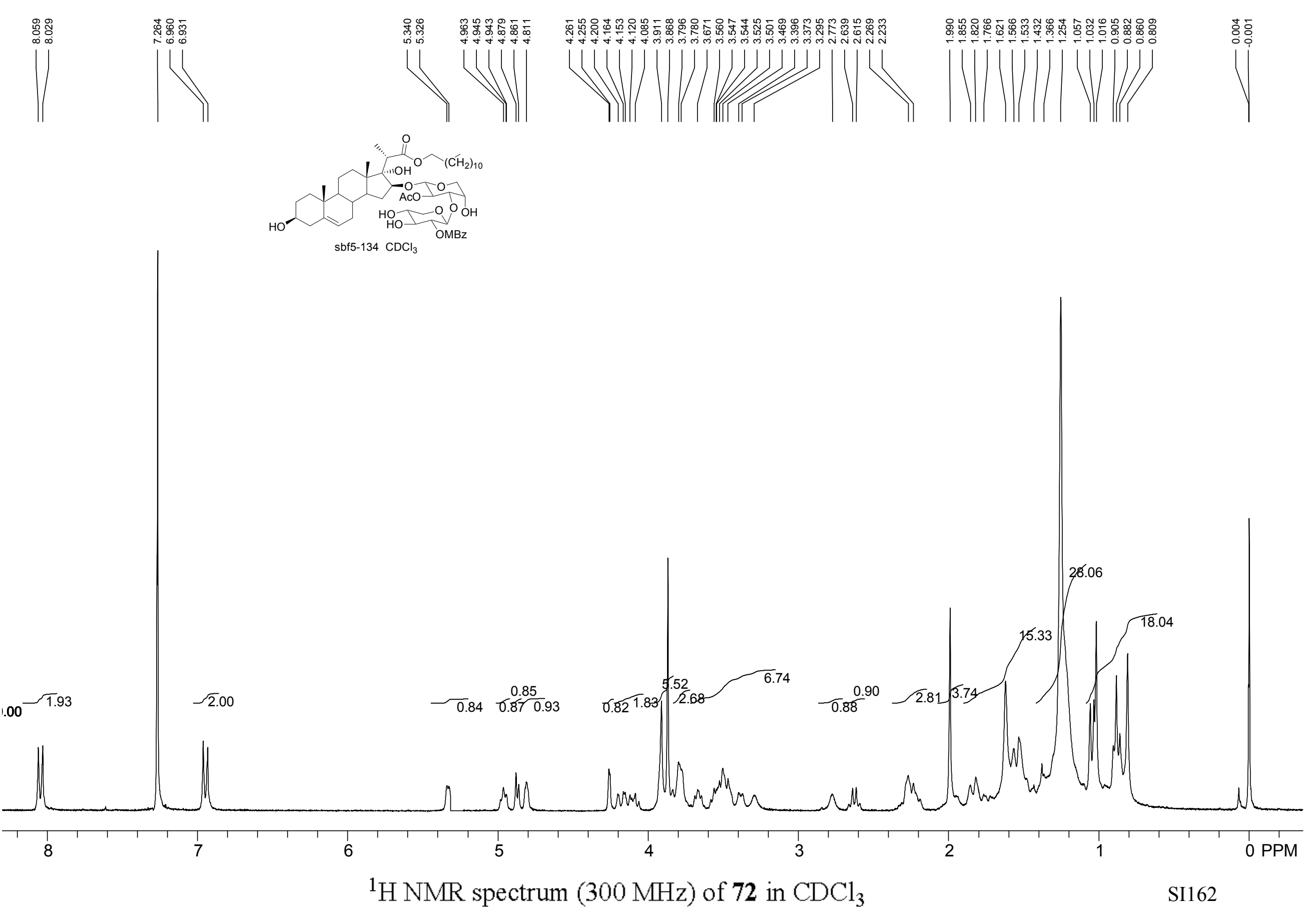



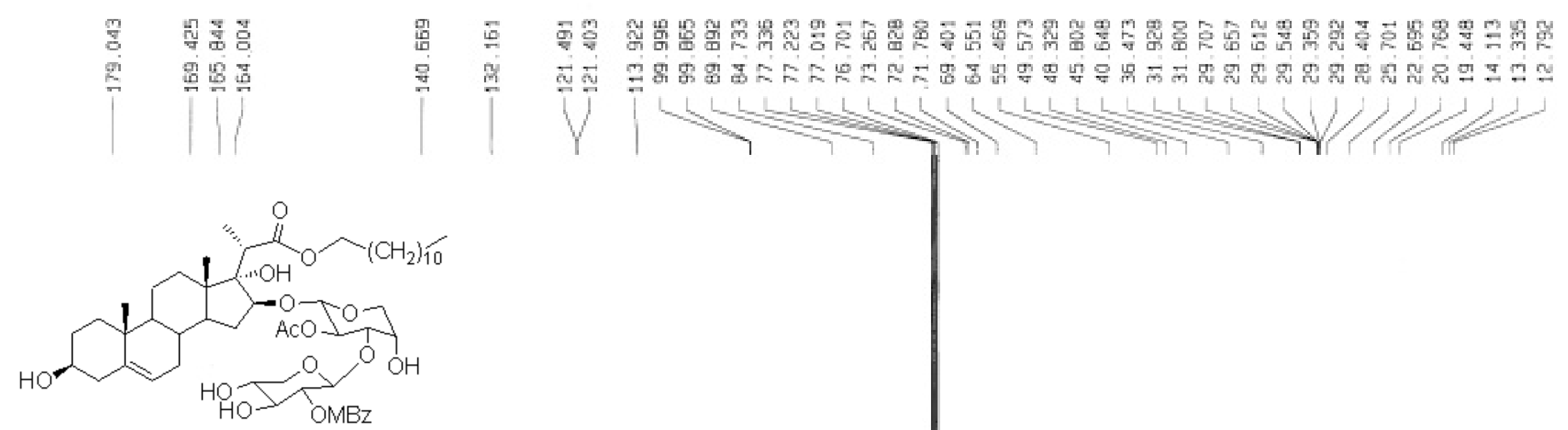

$$
\mathrm{C}_{54} \mathrm{H}_{82} \mathrm{O}_{16}
$$

Exact Mass: 986.56

Mol. Wt:: 987.22

C. $65.70 ; \mathrm{H}, 8.37 ; 0,25.93$

$\mathrm{CDCl}_{3}$

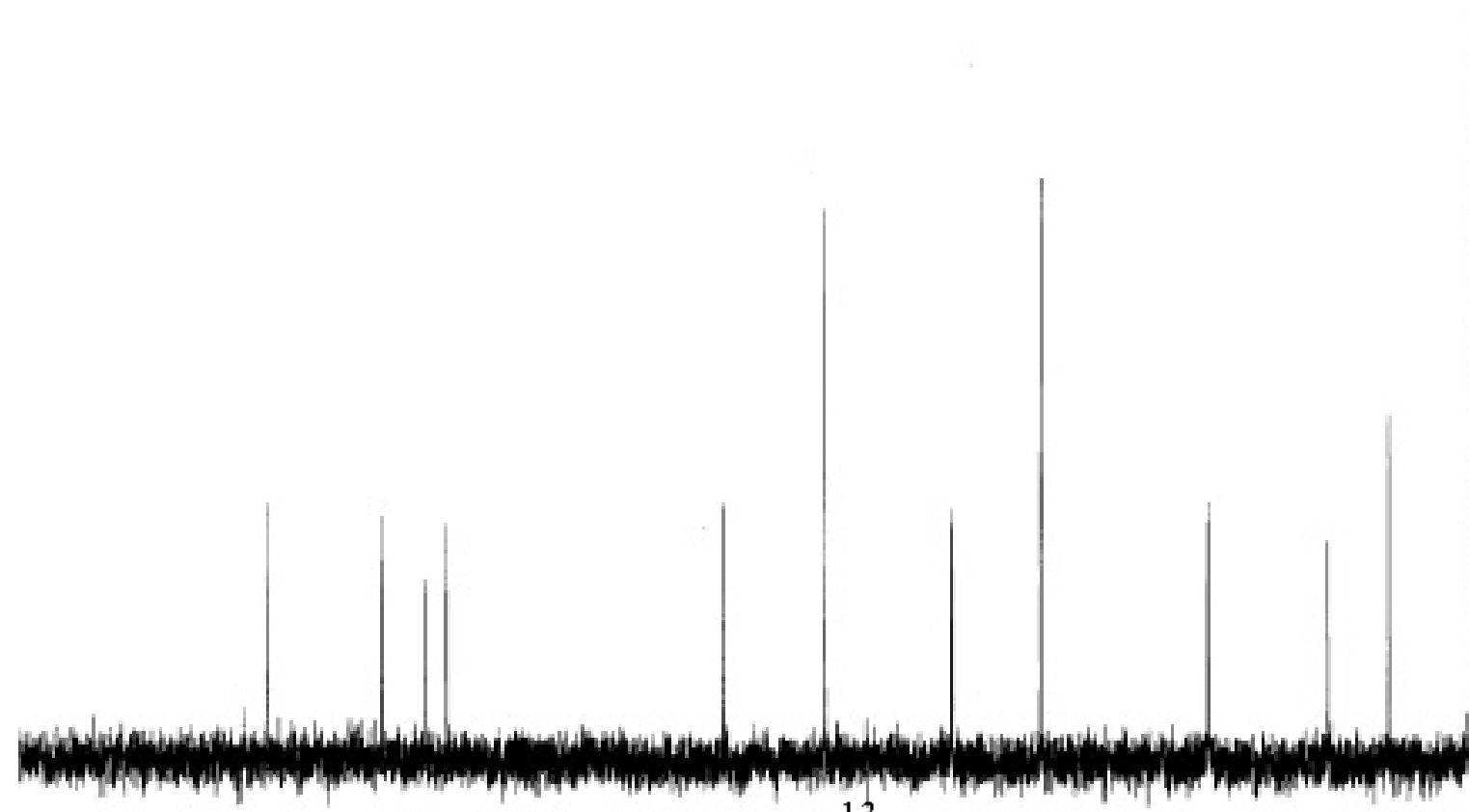

${ }^{13} \mathrm{CNMR}$ spectrum $(100 \mathrm{MHz})$ 


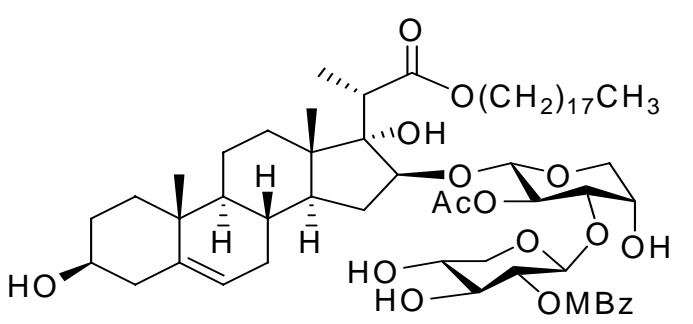

$\mathrm{C}_{60} \mathrm{H}_{94} \mathrm{O}_{15}$

Exact Mass: 1054.66

Mol. Wt.: 1055.38

C, 68.28; H, 8.98; O, 22.74

5.65 $3.28 \int^{3.03^{1.17} 3.62} 0.74$

1.59 2.793 .18 


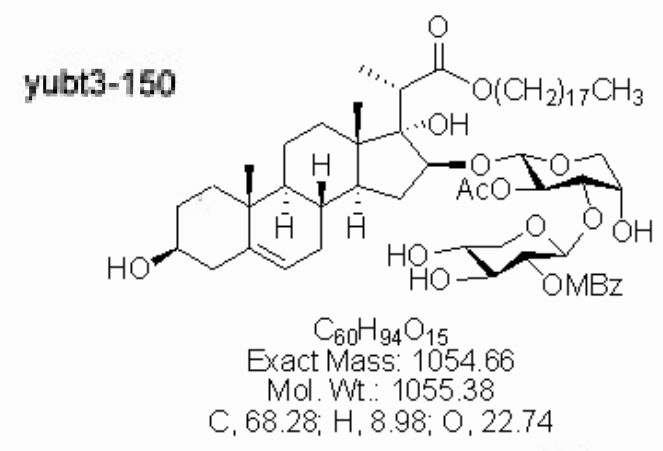




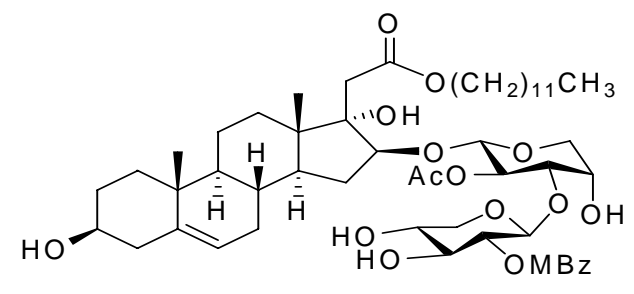

$\mathrm{C}_{53} \mathrm{H}_{80} \mathrm{O}_{15}$

Exact M ass: 956.55

C, 66.50; H, 8.42; O, 25.07

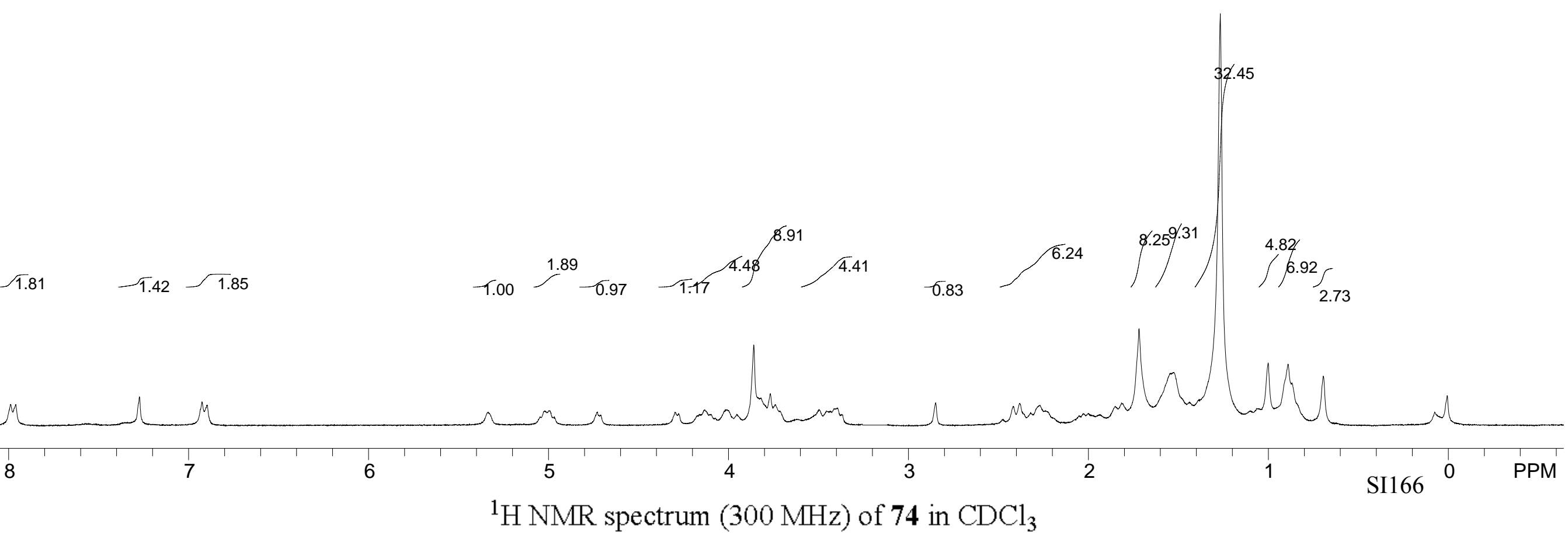




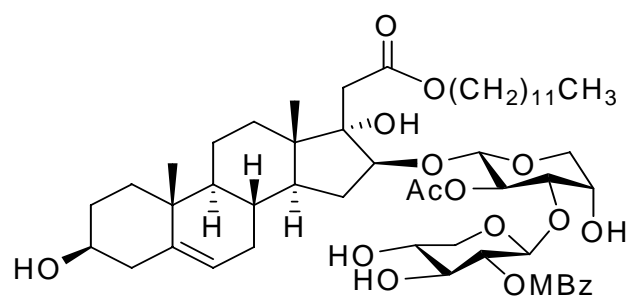

$\mathrm{C}_{53} \mathrm{H}_{80} \mathrm{O}_{15}$

Exact Mass: 956.55

Mol. Wt.: 957.19
$\mathrm{C}, 66.50 ; \mathrm{H}, 8.42 ; \mathrm{O}, 25.07$

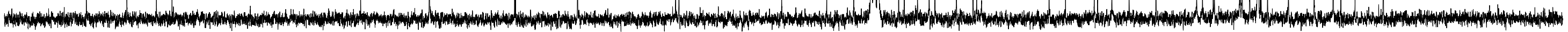

60

${ }^{13} \mathrm{CNMR}$ spectrum $\left(75 \mathrm{MHz}\right.$ ) of 74 in $\mathrm{CDCl}_{3}$
20

SI167
40

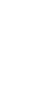



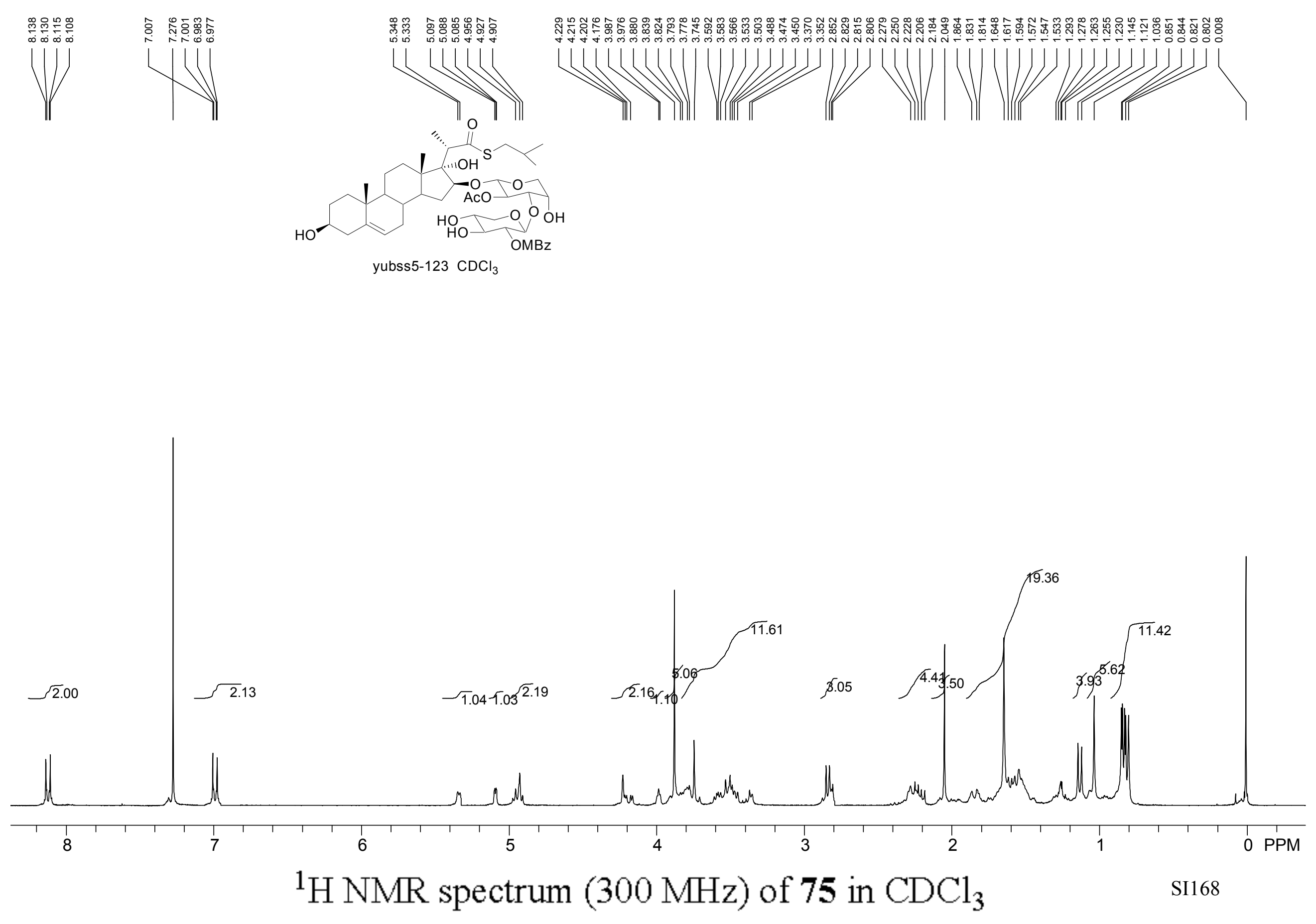
틈 $\stackrel{\text { g }}{\frac{9}{3}}$

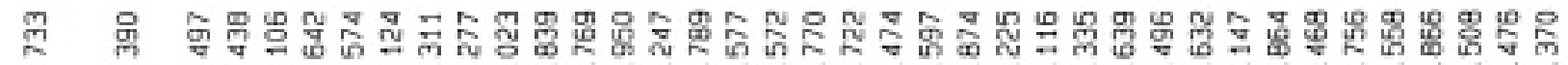
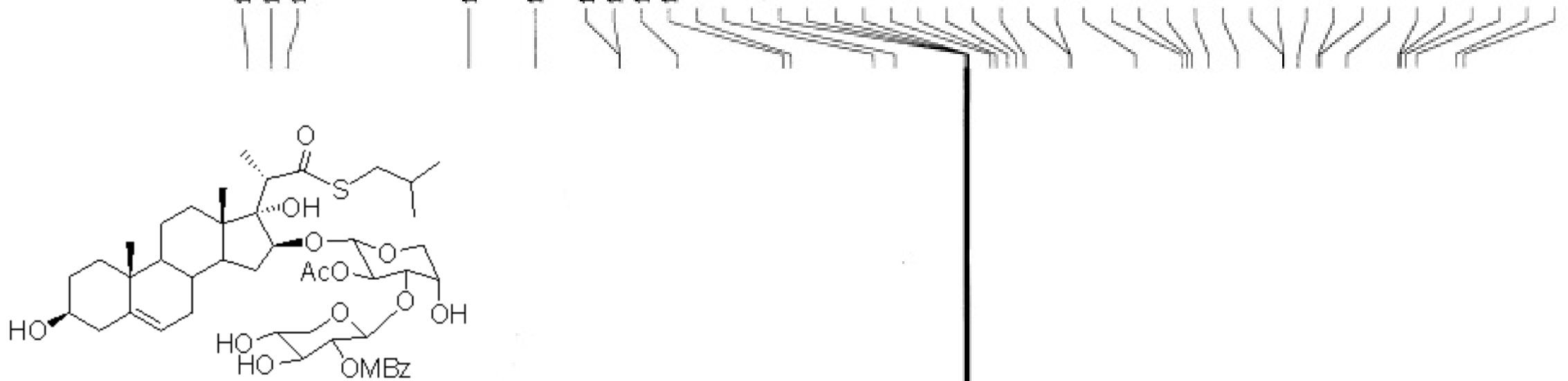

$\mathrm{C}_{46} \mathrm{H}_{66} \mathrm{O}_{15} \mathrm{~S}$

Exact Mass: 890.41

Mol. Wt:: 891.07

C, $62.00 ; H, 7.47 ; 0,26.93 ; S, 3.60$

$$
\mathrm{CDCl}_{3}
$$

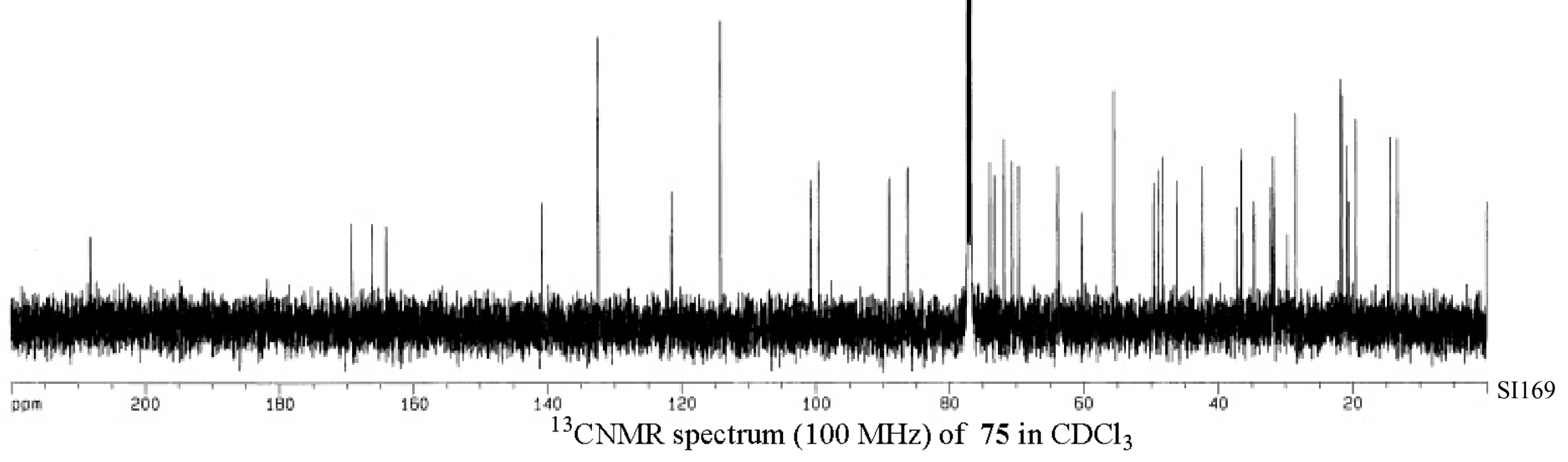



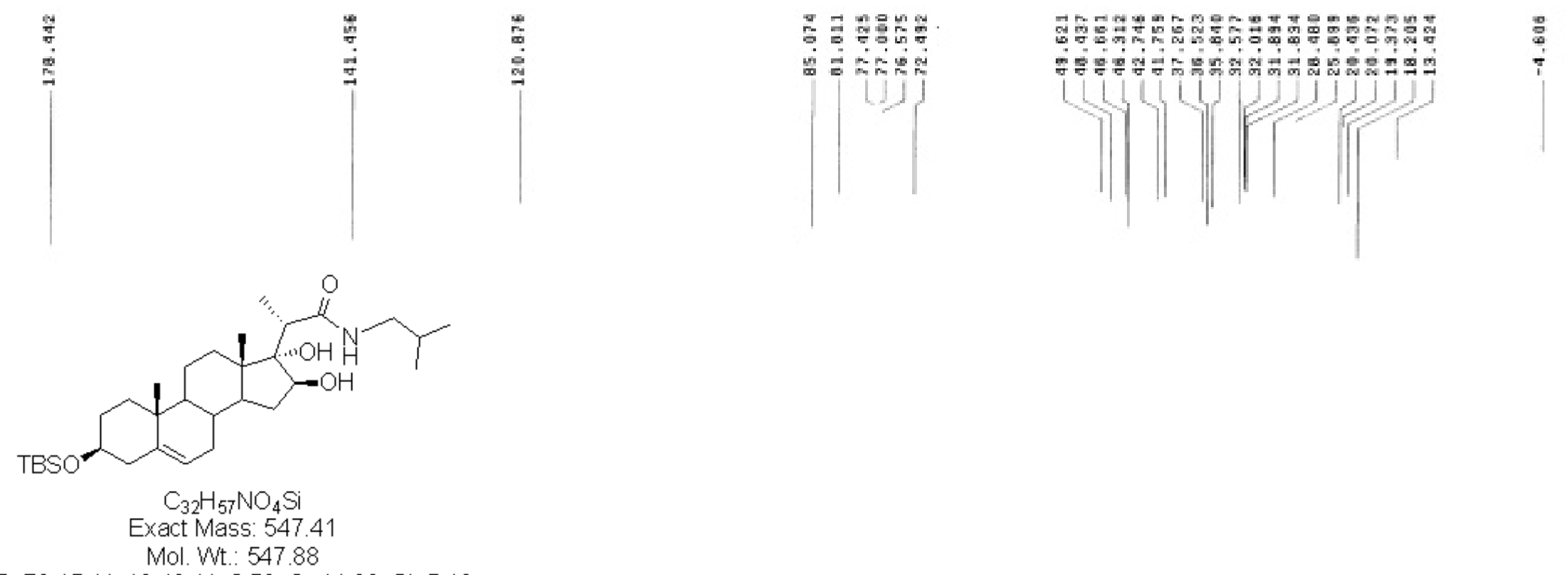

C. $70.15 ; H, 10.49 ; N, 2.56 ; 0,11.68 ; \mathrm{Si}, 5.13$

$\mathrm{CDCl}_{3}$

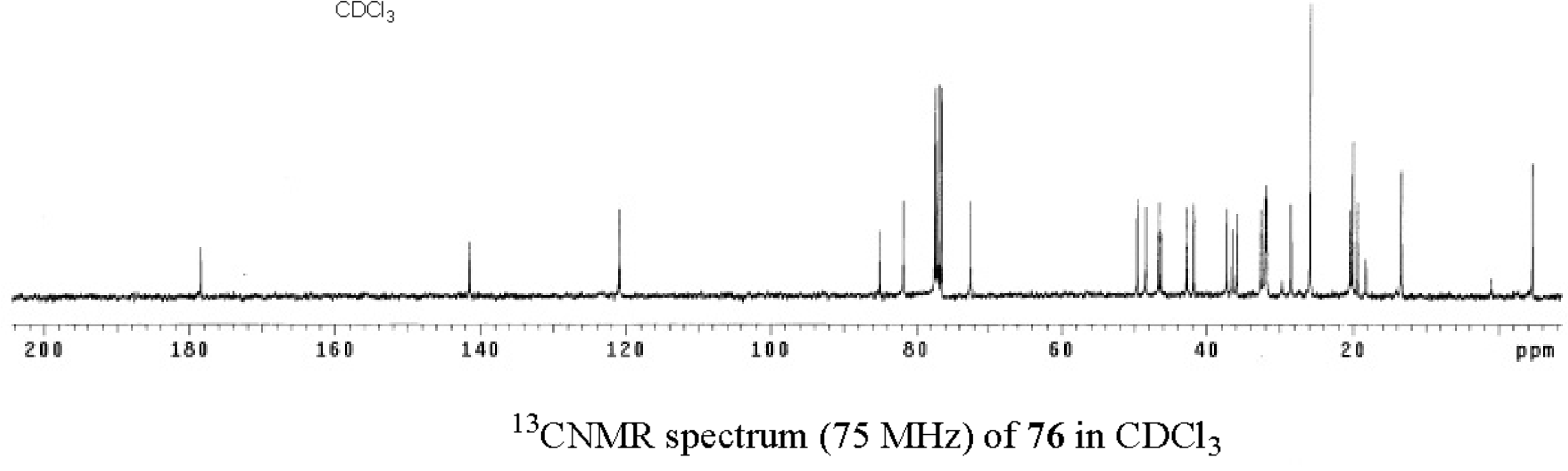



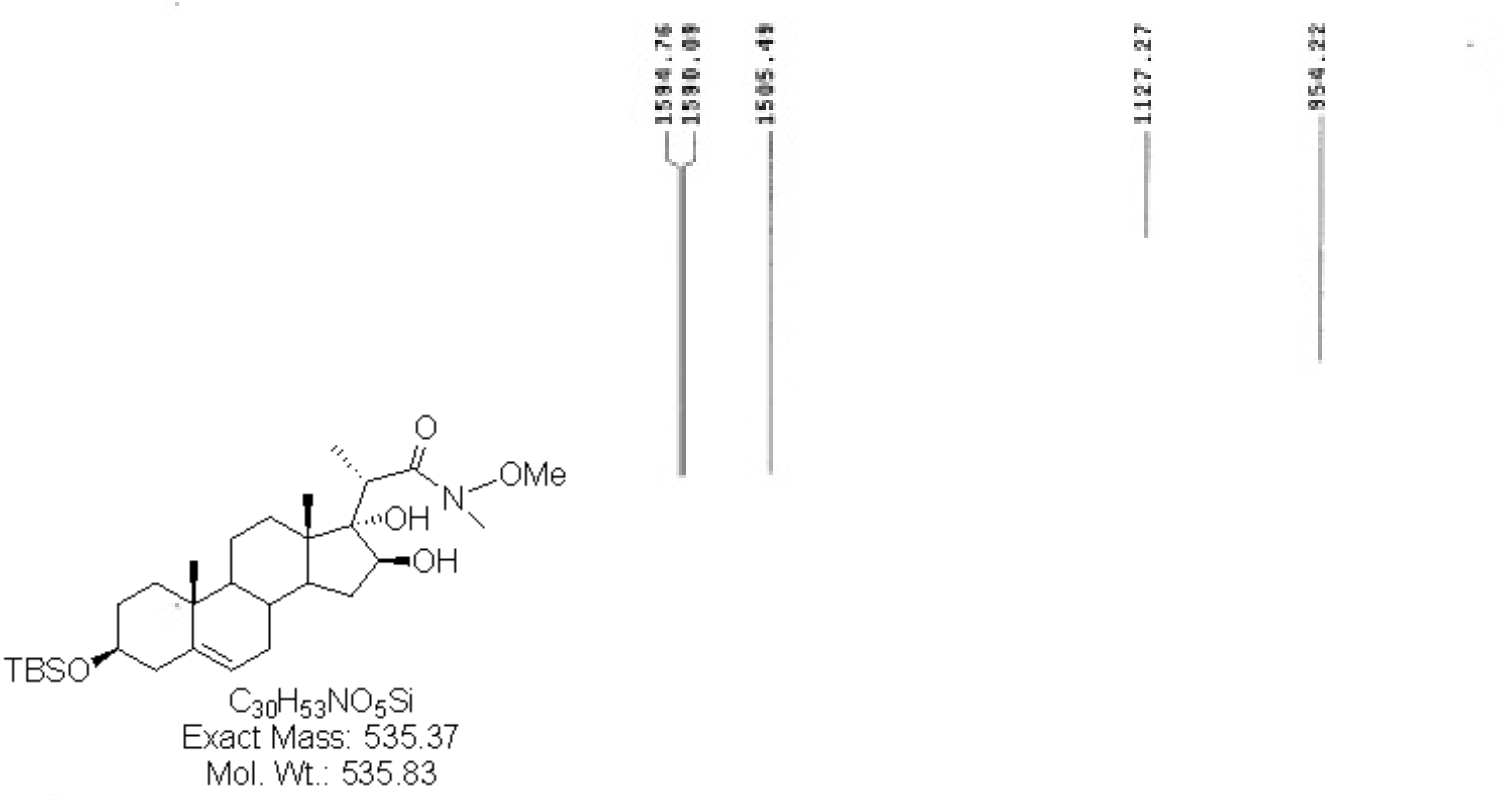

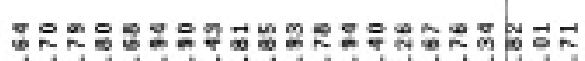

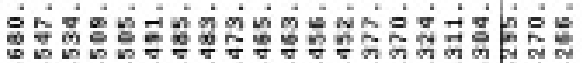
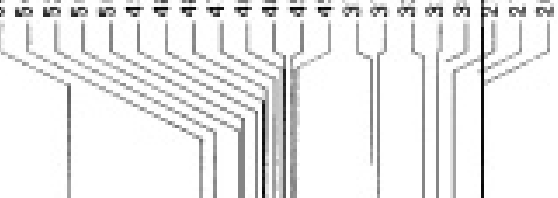

C. $67.25 ; \mathrm{H}, 9.97 ; \mathrm{N}, 2.61 ; \mathrm{O}, 14.93 ; \mathrm{Si}, 5.24$

$\mathrm{CDCl}_{3}$

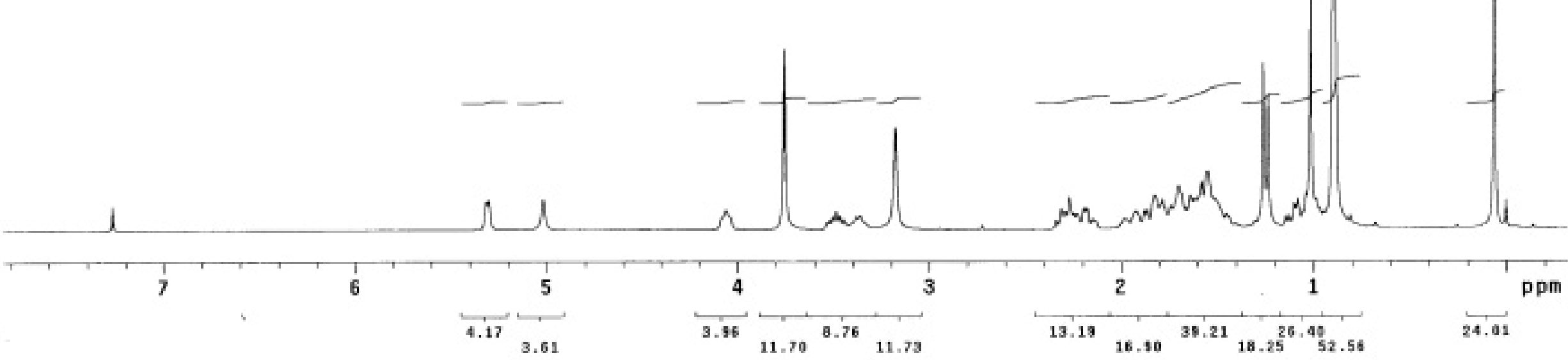

${ }^{1} \mathrm{H}$ NMR spectrum $(300 \mathrm{MHz})$ of 77 in $\mathrm{CDCl}_{3}$ 

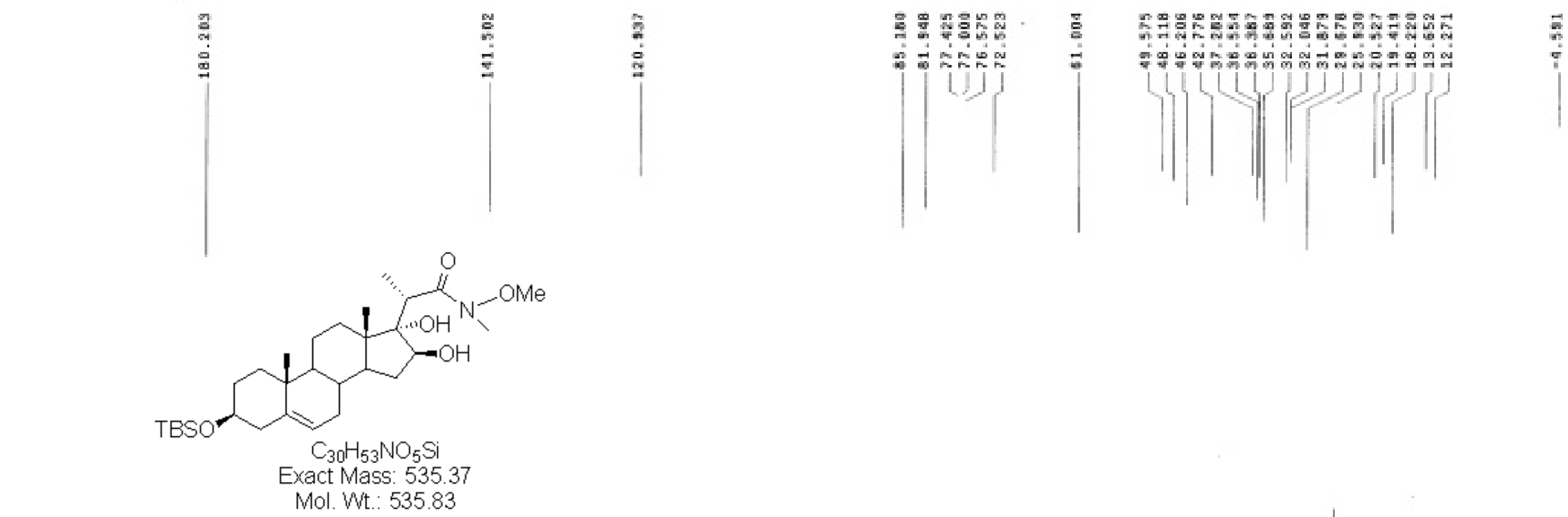

C. $67.25 ; \mathrm{H}, 9.97 ; \mathrm{N}, 2.61 ; \mathrm{O}, 14.93 ; \mathrm{Si}, 5.24$

$$
\mathrm{CDCl}_{3}
$$

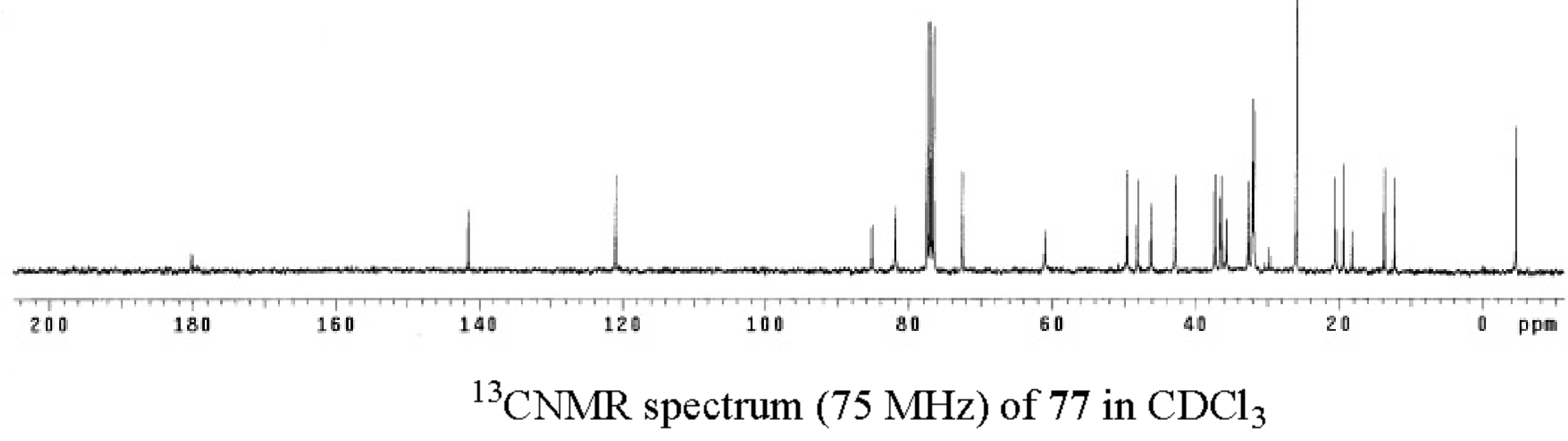




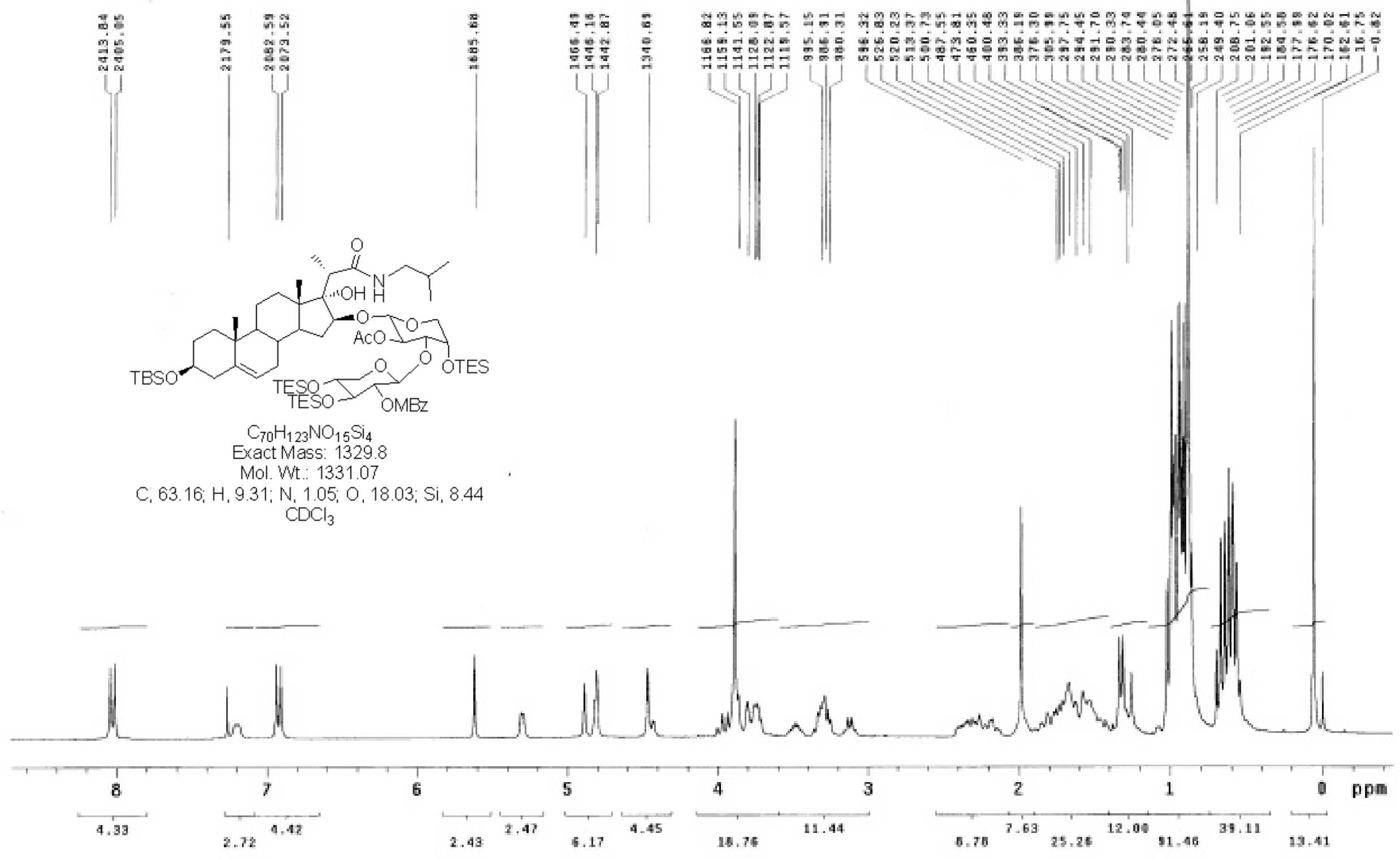

${ }^{1} \mathrm{H}$ NMR spectrum $(300 \mathrm{MHz})$ of 78 in $\mathrm{CDCl}_{3}$ 
C19
yubs55-75 in CAC 13

Pulse Sequence: 52 pul
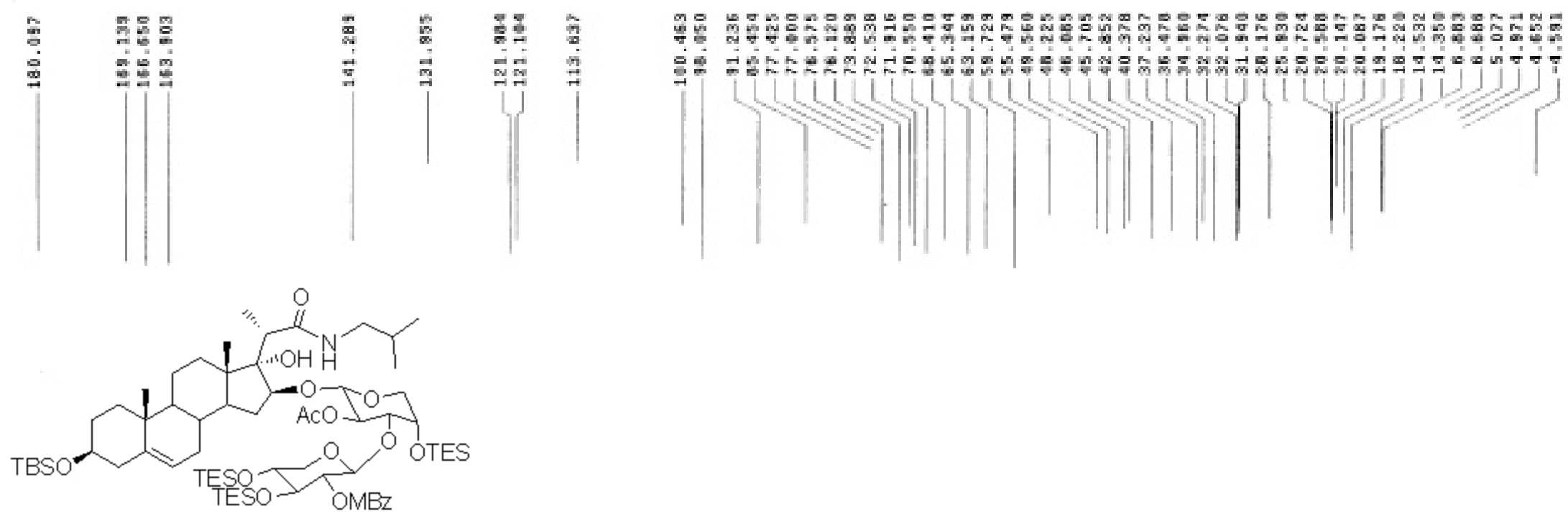

$\mathrm{C}_{70} \mathrm{H}_{123} \mathrm{NO}_{15} \mathrm{Si}_{4}$

Exact Mass: 1329.8

Mol. Wt: 1331.07

C, $63.16 ; \mathrm{H}, 9.31 ; \mathrm{N}, 1.05 ; 0,18.03 ; \mathrm{Si}, 8.44$

$\mathrm{CDCl}_{3}$

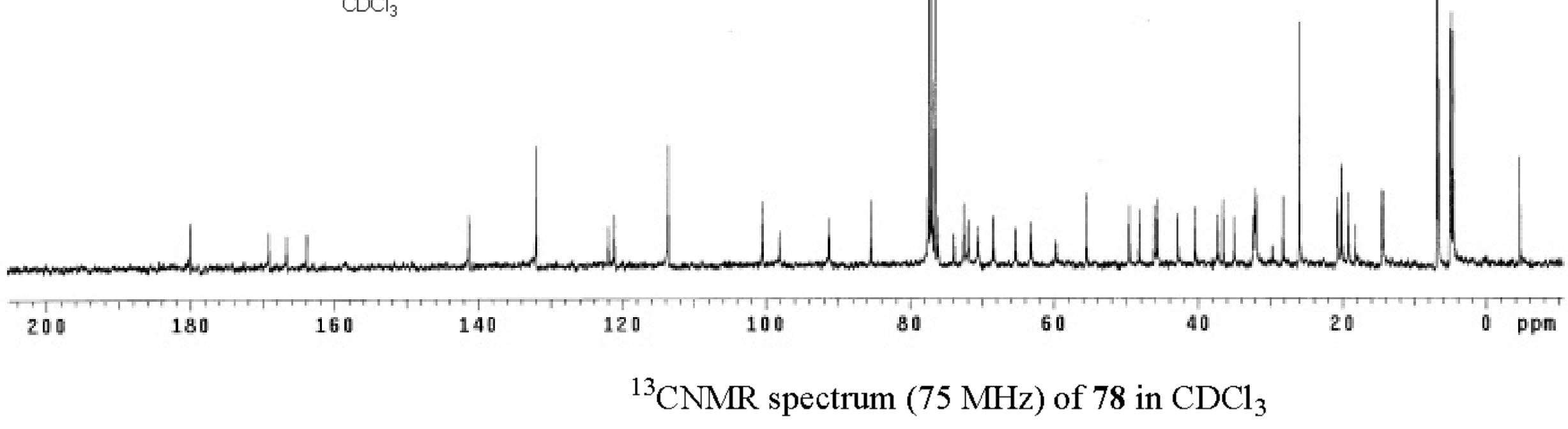



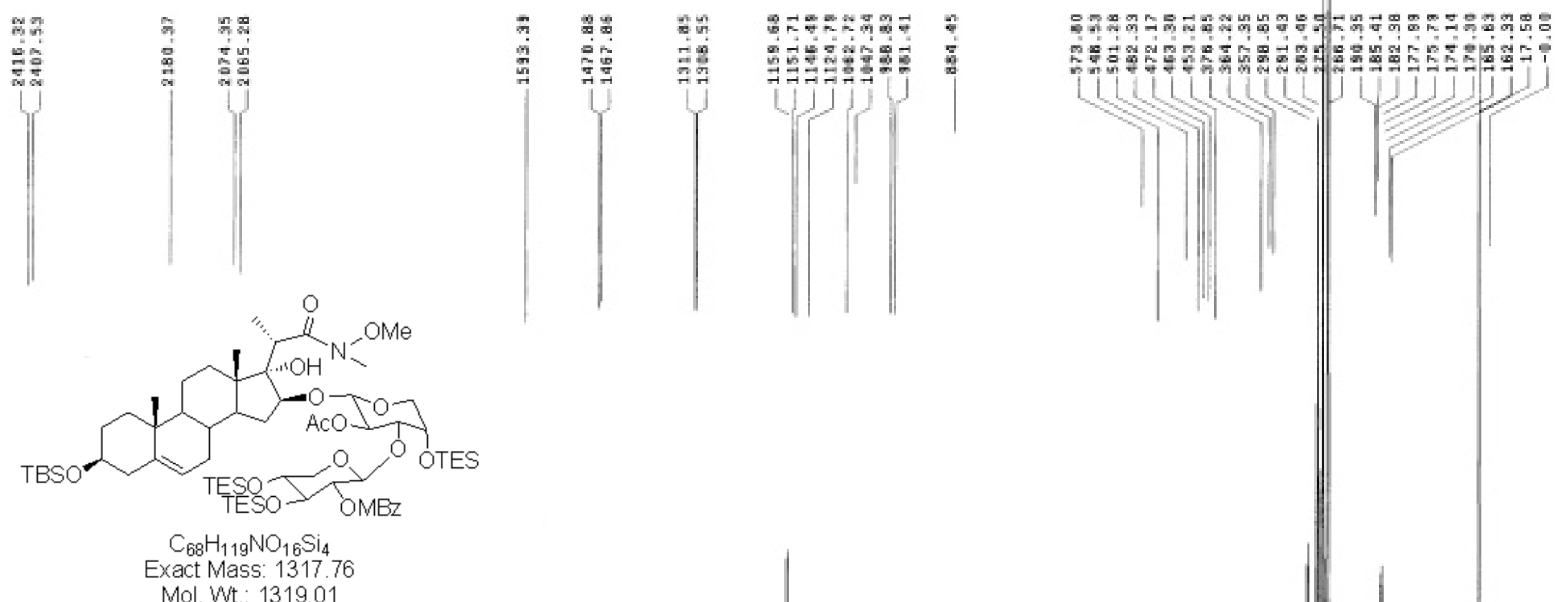

$\mathrm{CDCl}_{3}$

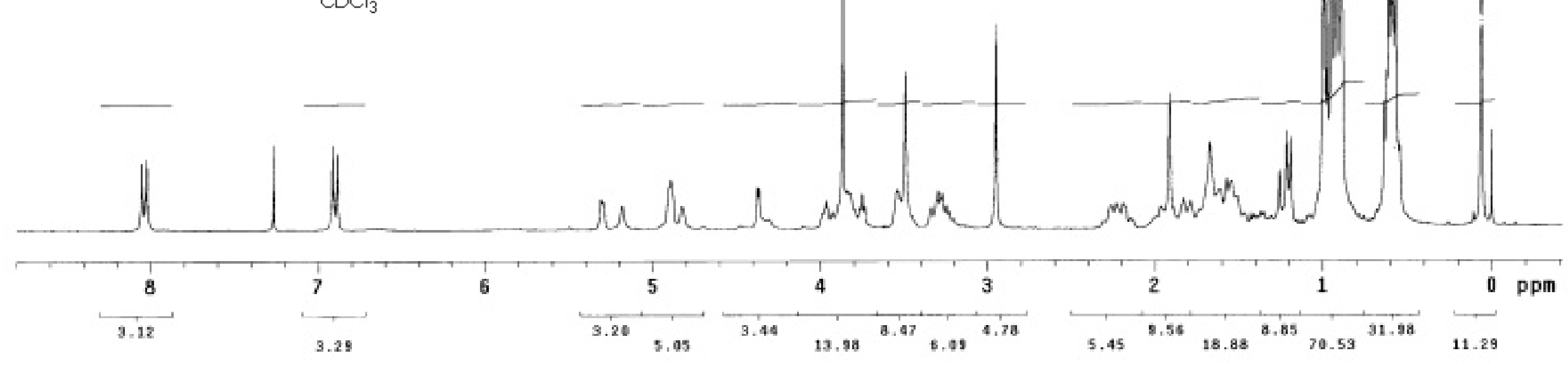

${ }^{1} \mathrm{H}$ NMR spectrum (300 MHz) of 79 in $\mathrm{CDCl}_{3}$ 

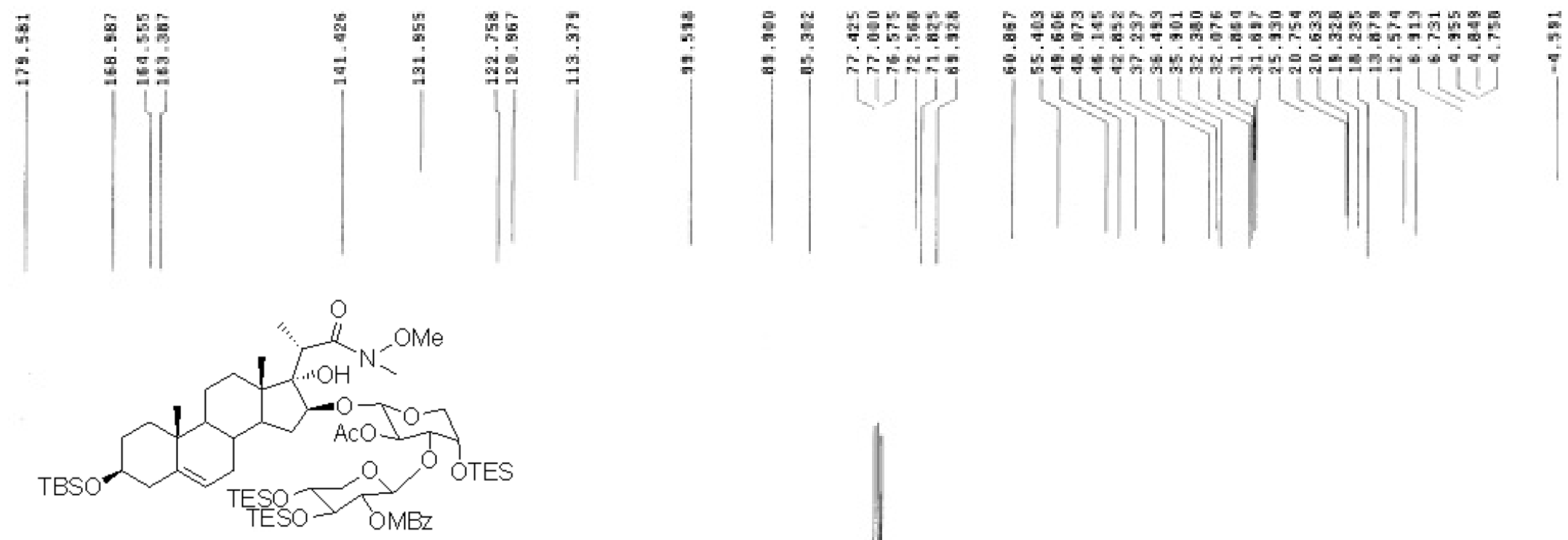

$\mathrm{C}_{68} \mathrm{H}_{119} \mathrm{NO}_{16} \mathrm{Si}_{4}$

Exact Mass: 1317.76

Mol. Wt.: 1319.01

C. $61.92 ; H, 9.09 ; N, 1.06 ; 0,19.41 ;$ Si, 8.52 $\mathrm{CDCl}_{3}$

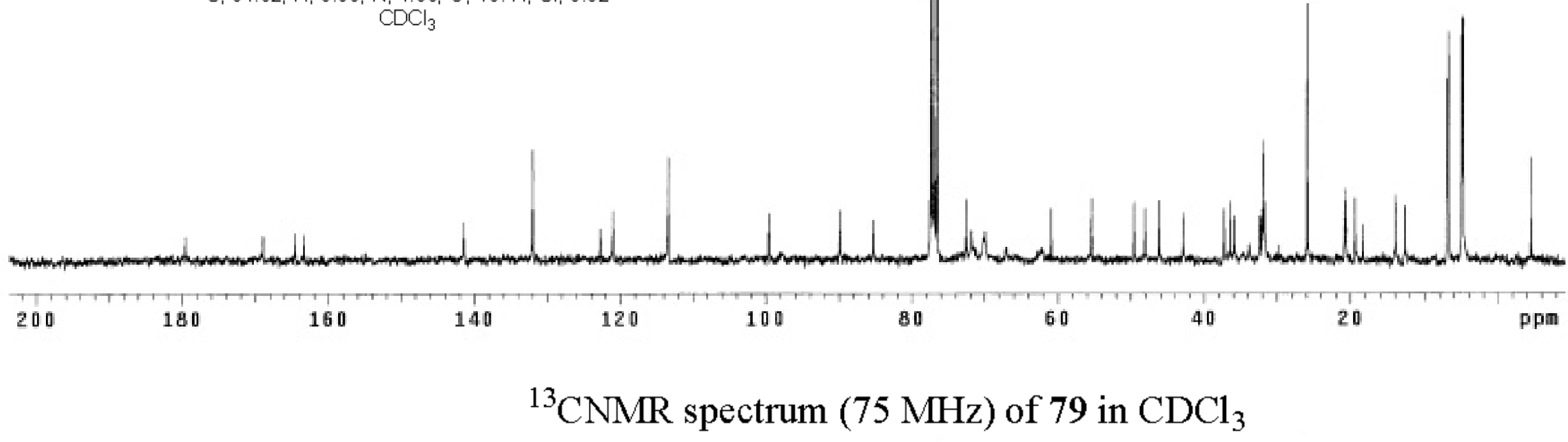



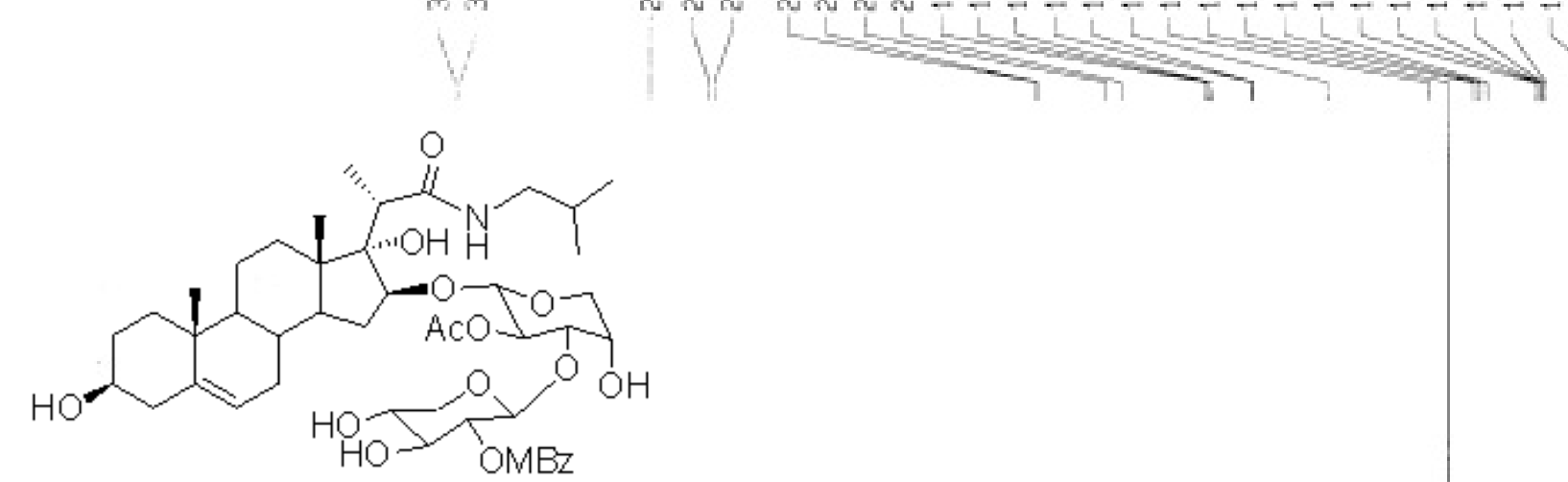

$\mathrm{C}_{46} \mathrm{H}_{67} \mathrm{NO}_{1}$

Exact Mass: 873.45

Mol. Wt: 874.02

C, $63.21 ; H, 7.73 ; N, 1.60 ; 0,27.46$ $\mathrm{CDCl}_{3}$

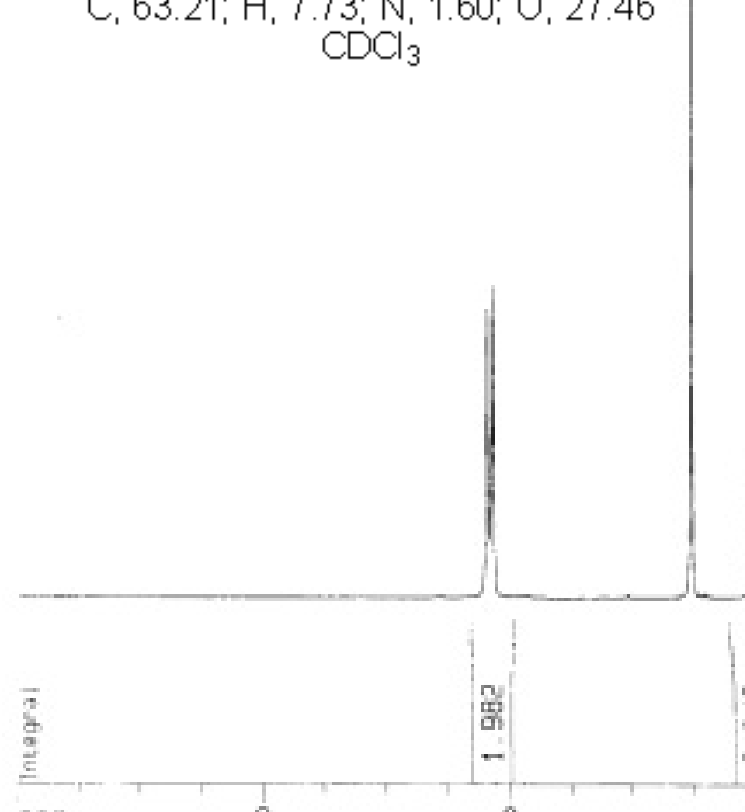

गु 

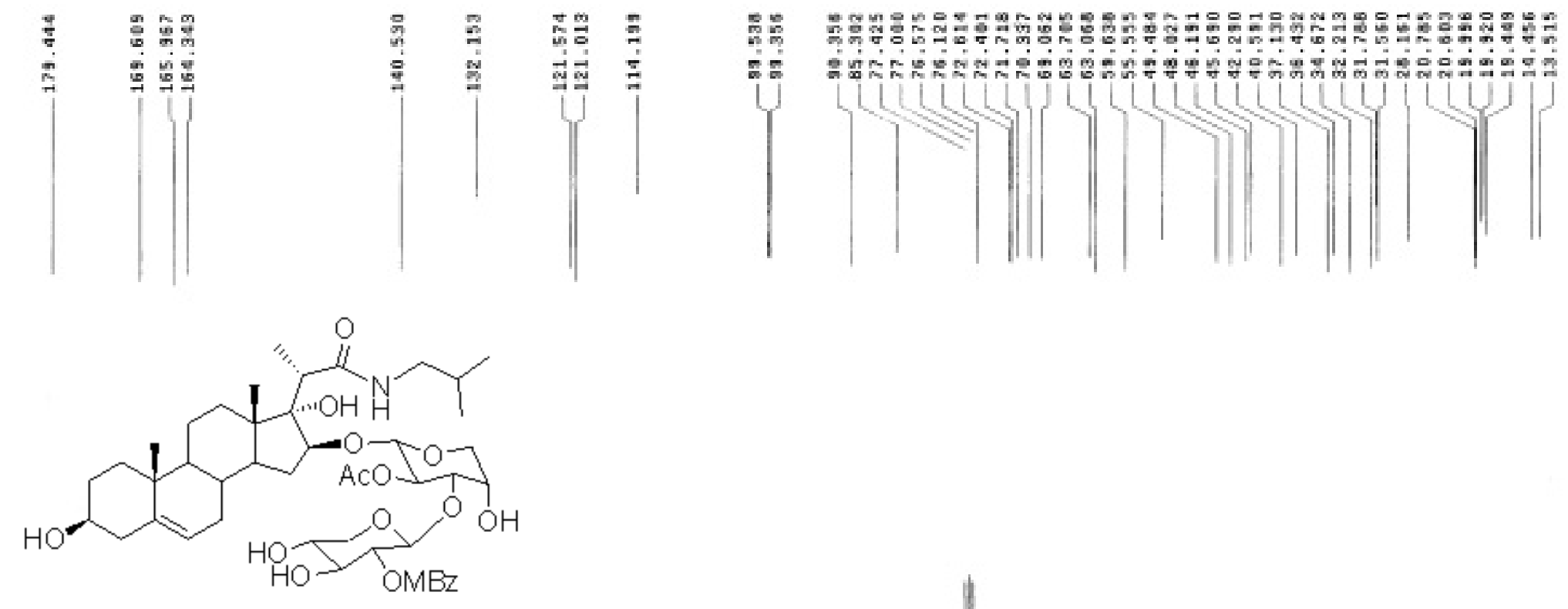

$\mathrm{C}_{46} \mathrm{H}_{67} \mathrm{NO}_{15}$

Exact Mass: 873.45

C. $63.21 ; H, 7.73 ; N, 1.60 ; O, 27.46$

$\mathrm{CDCl}_{3}$

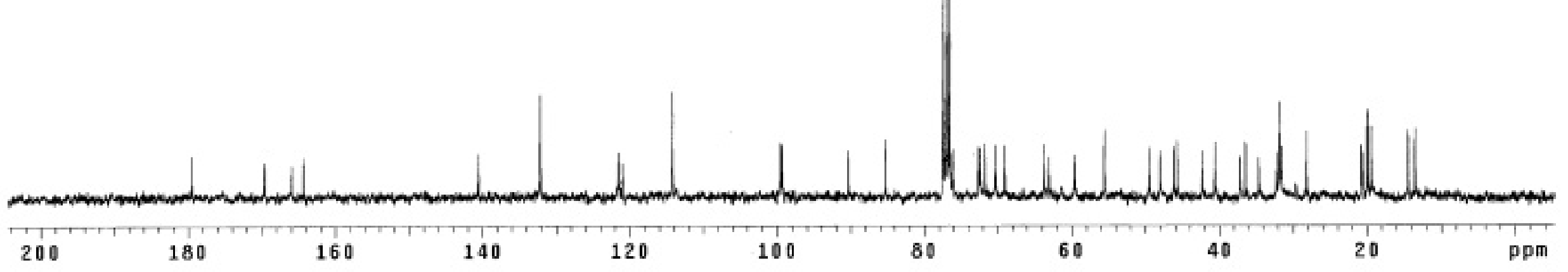

${ }^{13} \mathrm{CNMR}$ spectrum $(75 \mathrm{MHz})$ of 80 in $\mathrm{CDCl}_{3}$ 


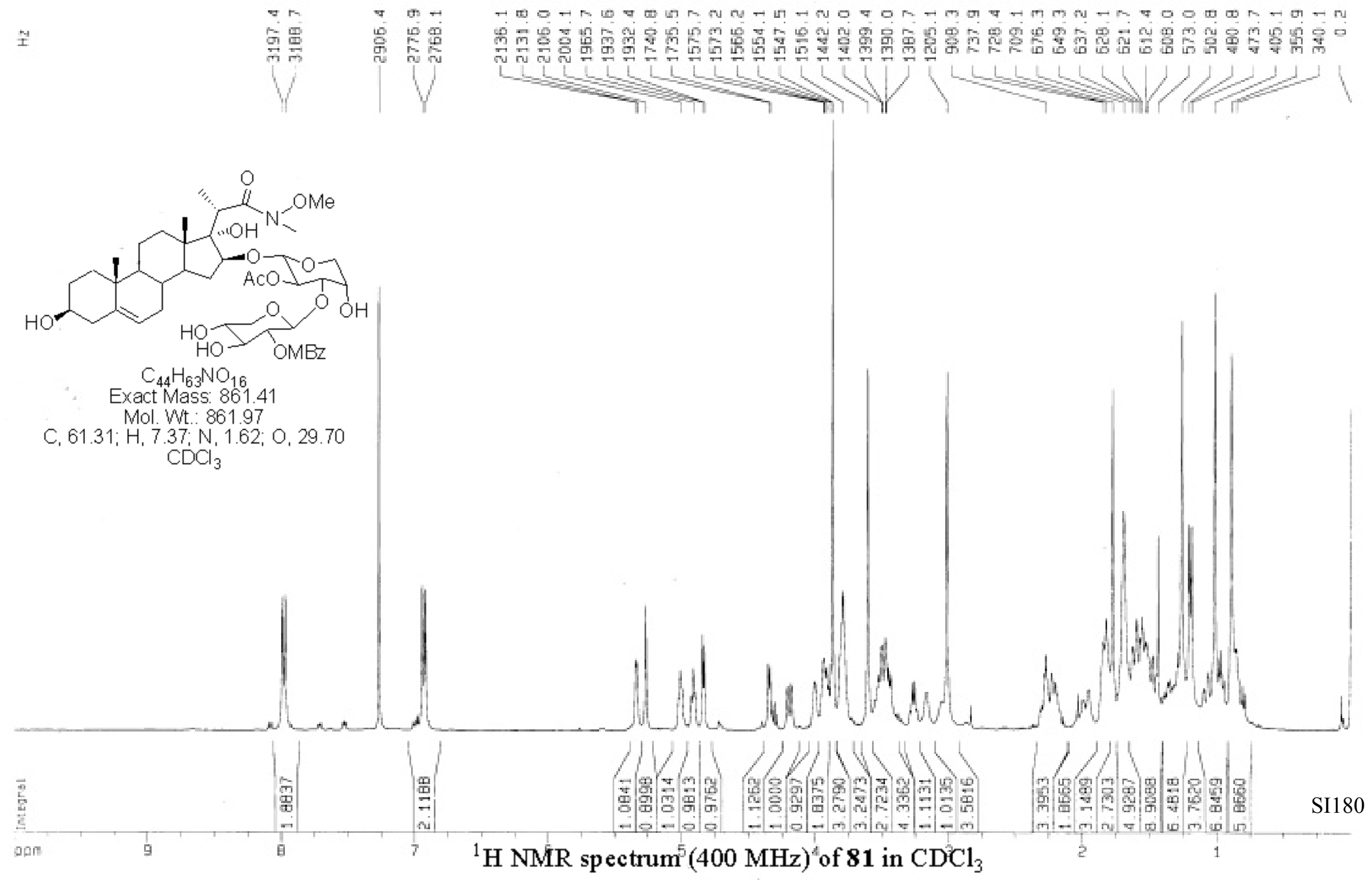




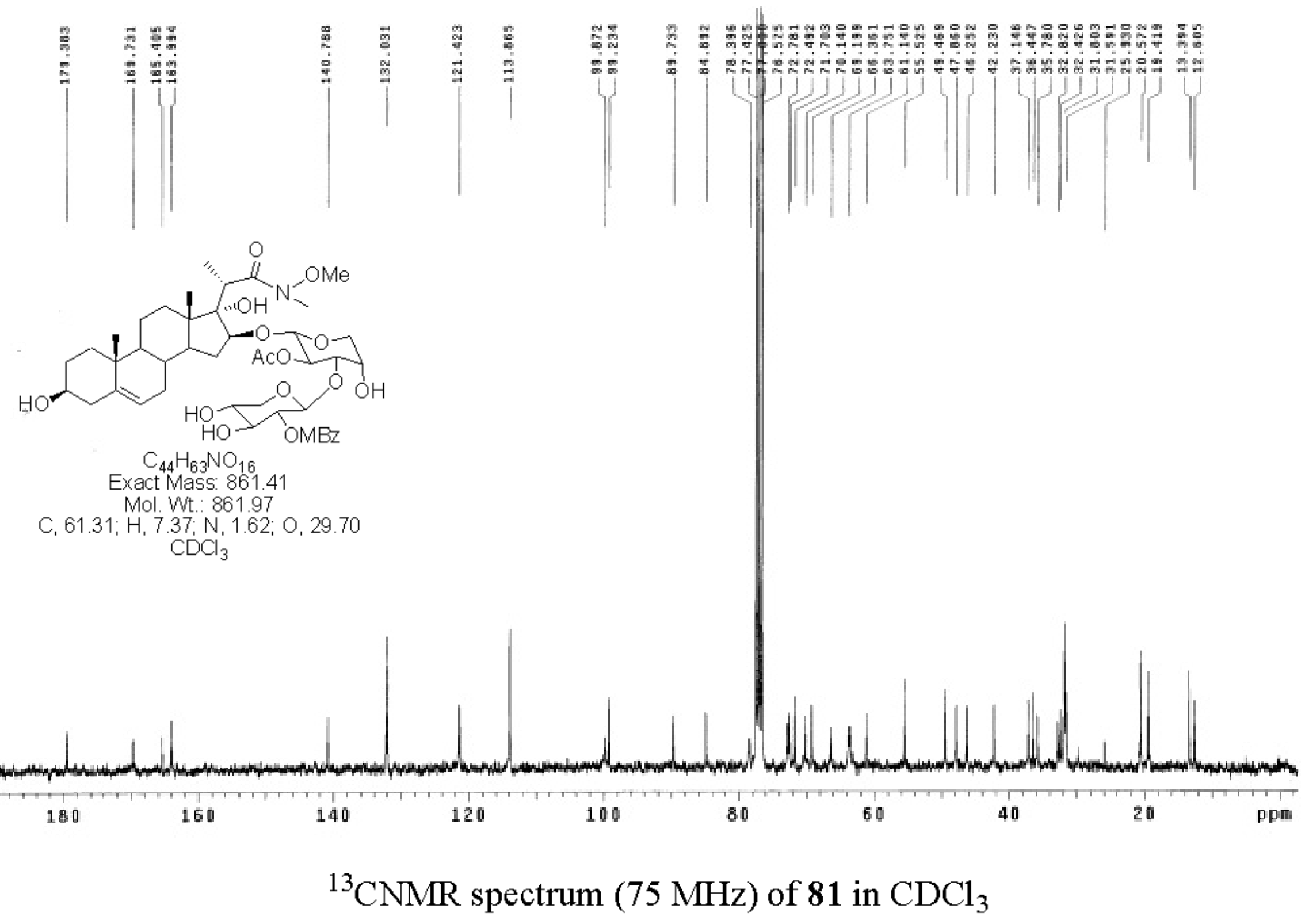


至

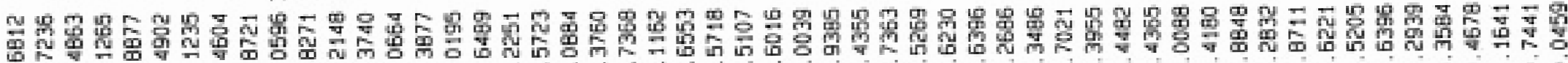

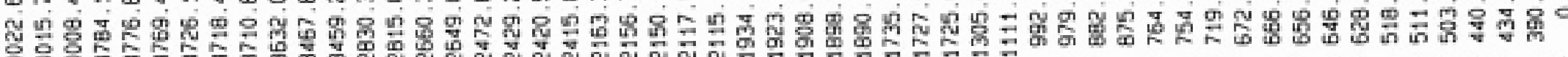
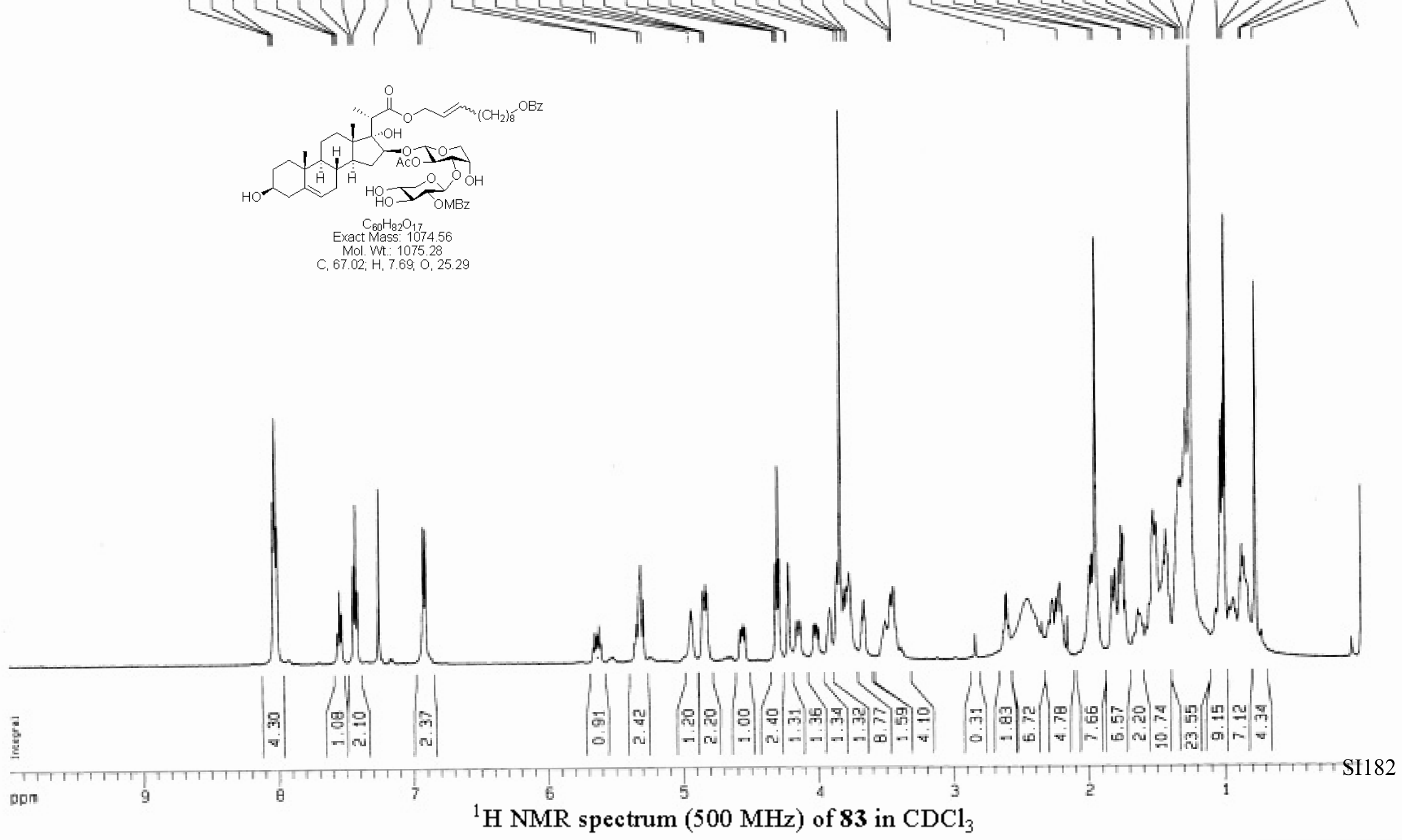


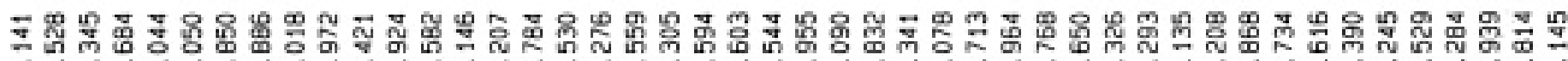

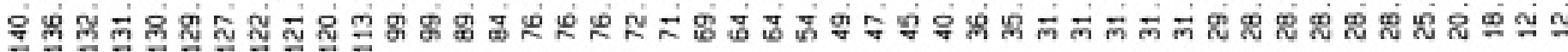

1111
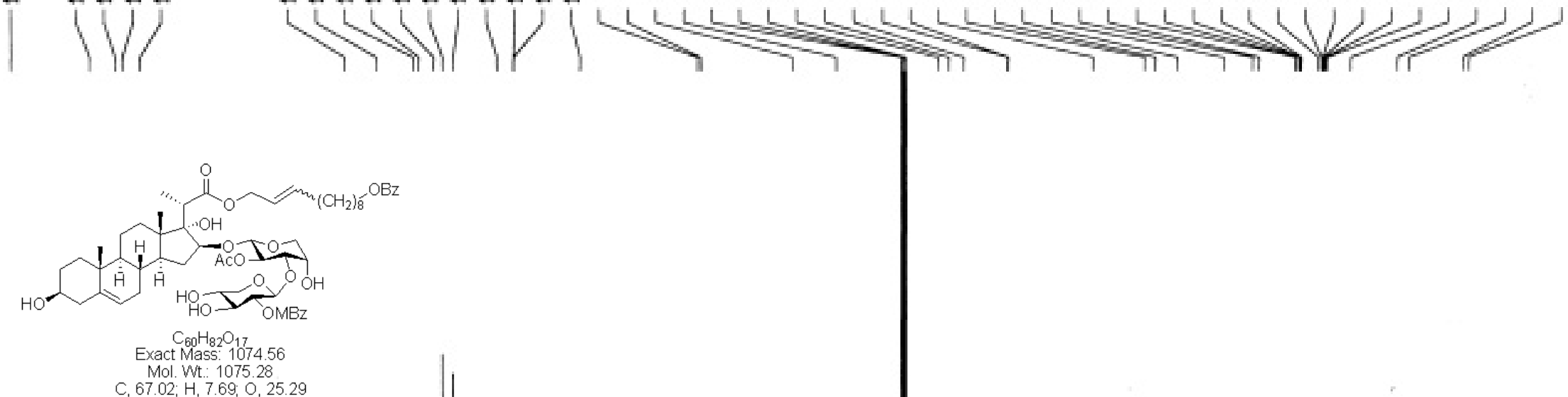

Mol. Wt: 107528

C, $67.02 ; H, 7.69 ; 0,25.29$ 


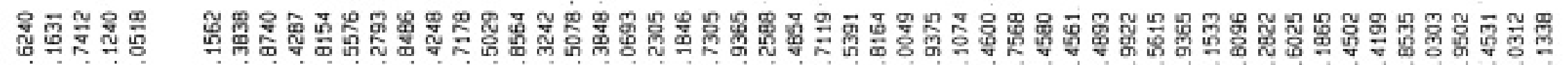

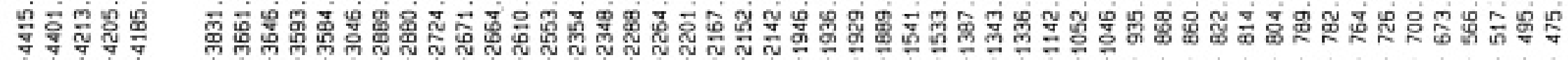

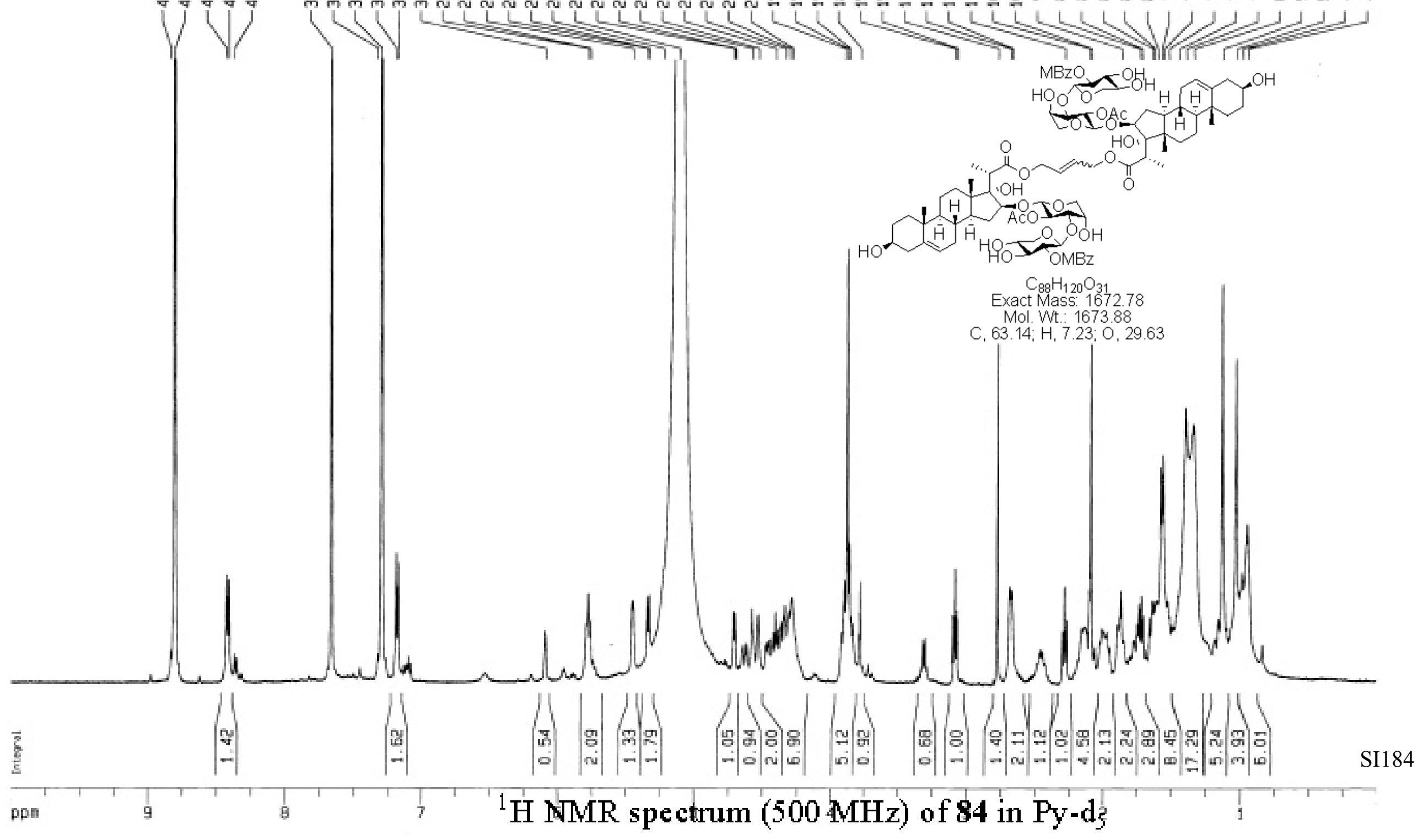




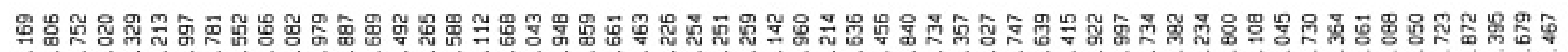

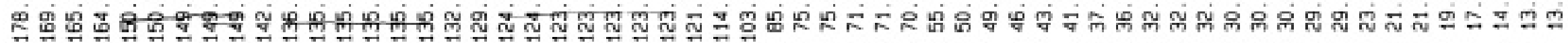

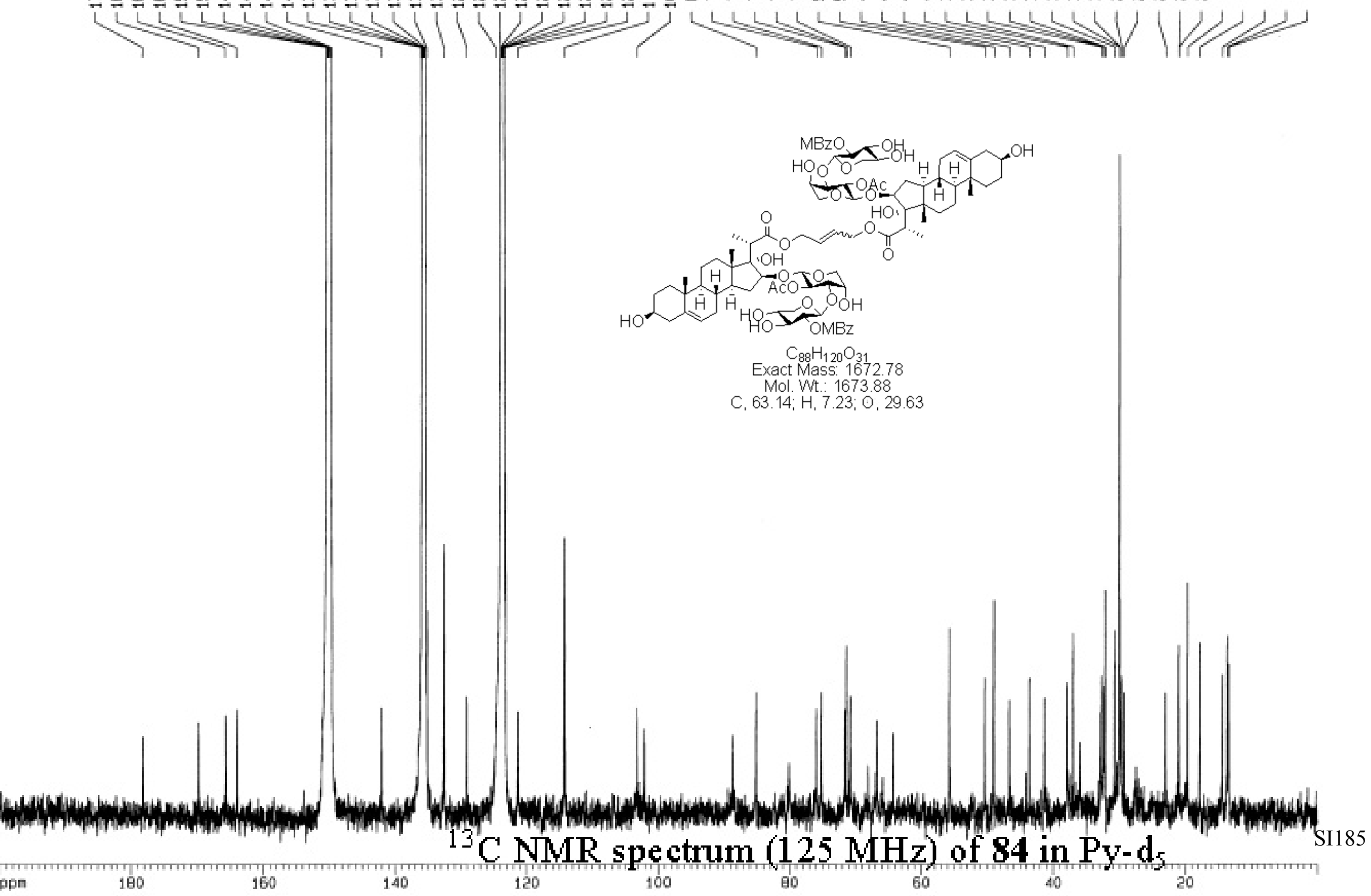




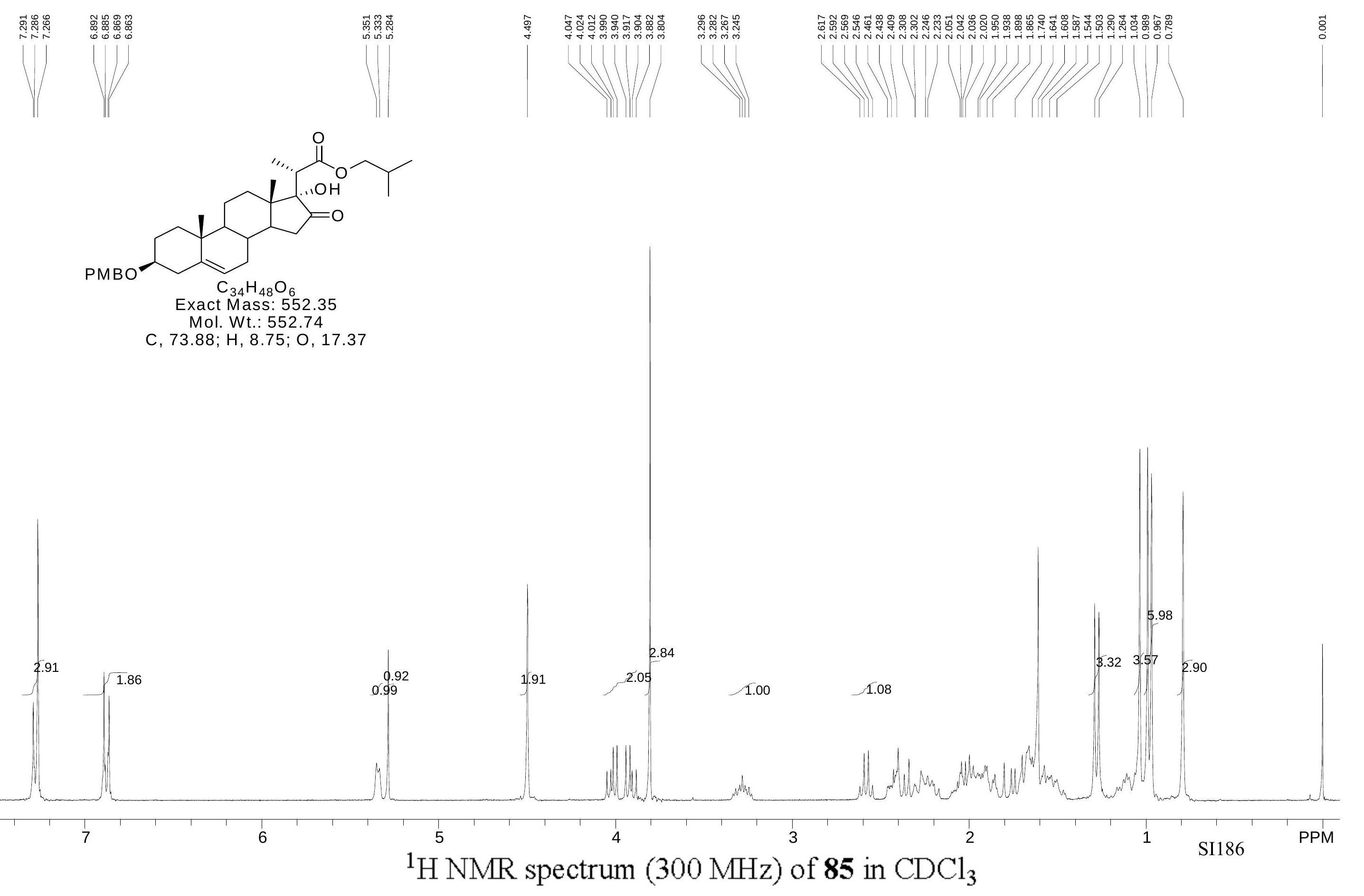




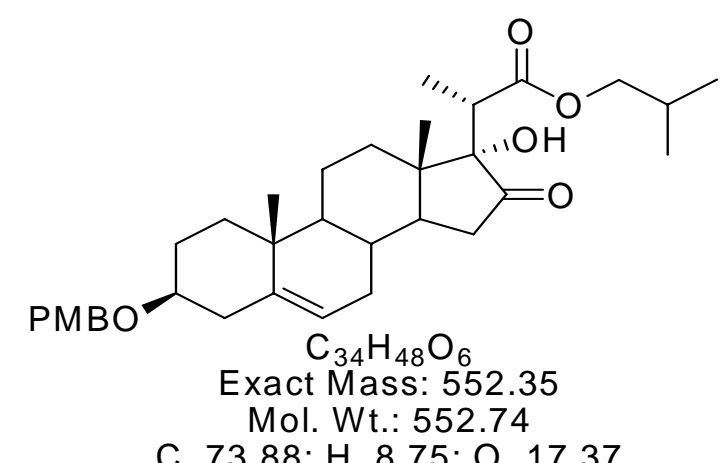

C, $73.88 ; \mathrm{H}, 8.75 ; \mathrm{O}, 17.37$ 